\title{
annual report 1980
}

\section{nuclear physics laboratory}

uI rsity of washington 


\section{DISCLAIMER}

This report was prepared as an account of work sponsored by an agency of the United States Government. Neither the United States Government nor any agency Thereof, nor any of their employees, makes any warranty, express or implied, or assumes any legal liability or responsibility for the accuracy, completeness, or usefulness of any information, apparatus, product, or process disclosed, or represents that its use would not infringe privately owned rights. Reference herein to any specific commercial product, process, or service by trade name, trademark, manufacturer, or otherwise does not necessarily constitute or imply its endorsement, recommendation, or favoring by the United States Government or any agency thereof. The views and opinions of authors expressed herein do not necessarily state or reflect those of the United States Government or any agency thereof. 


\section{DISCLAIMER}

Portions of this document may be illegible in electronic image products. Images are produced from the best available original document. 


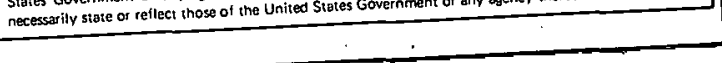

\title{
ANNUAL REPORT
}

\author{
Nuclear Physics Laboratory \\ University of Washington \\ September, 1980
}

Supported in part by the United Ștates Department of Energy under contract EY-77-C-06-1388

This report was prepared as an account of work sponsored by the United States Government. Neither the United States nor the United States Department of Energy, nor any of their employees, makes any warranty, express or implied, or assumes any legal liability or responsibility for the accuracy, completeness or usefulness of any information, apparatus, product or process disclosed, or represents that its use would not infringe. privately-owned rights. 
I NTRODUCTION

This Annual Report covers the period from April 1979 to April 1980 and includes all work done in the Nuclear Physics Laboratory. The majority of these projects are supported by our Department of Energy contract, but experiments and therapy performed by the Medical School and problems of applied physics pursued by investigators from the College of Engineering and by outside users are also included.

This last year has been an eventful one. We have asked Bob Vandenbosch to serve as Director of the Nuclear Physics Laboratory and he has accepted. Unfortunately one of his first acts was to ask me to be editor of the Annual Report again. Our proposal to DOE for an $18 \mathrm{MV}$ folded tandem accelerator was reviewed by NUSAC and was recommended for funding in FY 82. This very good news is unfortunately tempered by a dismal outlook for federal support for nuclear physics in the next few years. Ne hope that the financial picture will improve and that our proposed facility, which would be a powerful addition to the national accelerator complement, can become a reality. The $18 \mathrm{MV}$ tandem would provide intense, high quality, DC beams of polarized protons and deuterons, as well as the variety of light and heavy ions one can obtain from tandem accelerators. We believe that ancillary instrumentation and computer facilities recently developed or under construction will make it possible to attack a broad range of interesting types with the new machine.

During this last year a number of interesting discoveries have been made on our tandem accelerator which continued to $r$ un very well indeed. Some highlights of the last year's research follow.

Following last year's discovery of substantial Ml strength built on the ground state of the doubly magic $16_{0}$ nucleus, this year we have detected substantial $\mathrm{M} 1$ decays to the excited $\mathrm{O}_{2}^{+}(6.05 \mathrm{MeV})$ state from the 16.22 and $17.14 \mathrm{MeV}^{+}$, T1 states in ${ }^{16} \mathrm{O}$. We have also found decays of the $1^{+}, \mathrm{T}=1 \quad(10.32$ $\mathrm{MeV}$ ) level in ${ }^{40} \mathrm{Ca}$ to the $\mathrm{O}_{2}{ }^{+}$and $21^{+}$final states, with reduced strengths (relative to the ground-state decay) very similar to those found in 160 , and a ground-state strength compatible with electron scattering results and much smaller than previously believed. We have completed our study of the Ml $y$-decay of the $4^{-}, T=1(18.98 \mathrm{MeV})$ "stretched particle-hole state" and its neighboring $3^{-}, \mathrm{T}=1$ (18.03) $\mathrm{MeV}$ state, with results for the $4^{-}$state in agreenent with 1 particle-1 hole shell model calculations. We have used the well-known M1/E2 $\mathrm{T}=3 / 2$ resonance in the ${ }^{12} \mathrm{C}\left(\mathrm{p}, \gamma_{0}\right)$ reaction to make the first unique amplitude and phase determination for El capture in the GDR region, with the result that d-wave capture doninates, as expected by the shel1 mode1. Finally, our new results for the $\mathrm{E} 2$ cross section in the $54_{\mathrm{Fe}\left(\alpha, \gamma_{0}\right)}^{58} \mathrm{Ni}$ reaction remove an outstanding discrepancy with $E 2$ strength inferred from a $58 \mathrm{Ni}\left(\alpha, \alpha^{\prime}, \alpha_{0}\right)^{54} \mathrm{Fe}$ decay-coincidence experiment. 
The excitation function for the total angle-integrated yield of the ${ }^{28}$ Si $2^{-1}$ $\left(E_{X}=1.78 \mathrm{MeV}\right)$ state in the ${ }^{12} \mathrm{C}+{ }^{28} \mathrm{Si}$ reaction has been measured and found to vary smoothly with energy, in contrast to the behavior of the back-angle differential cross section. This result is inconsistent with previous resonance interpretations of the back-angle differential cross sections.

The $160+160$ system has been studied to deternine if collisions arising from low impact parameters contribute to the deep inelastic scattering exit channel $160^{*}+10^{*}$. This experiment was motivated by time dependent Hartree-Fock (TDHF) theory calculations, which predict that at elevated bombarding energies nearly central collisions between two heavy ions do not lead to fusion as would otherwise he expected. Prcviously no direcl experimental evidence was available to test this aspect of TDHF theory. Dur preliminary experimeilial resules indicate a stzable croso scction for large energy losses which peaks at $\theta_{\mathrm{cm}}=90^{\circ}$, an angle much larger than the grazing angle of $20^{\circ}$. The large deflection is inconsistent with an interpretation that the observed yields arlse from the outer partial waves, but is qualitatively consistent with expectations for low $\ell$ waves as predicted by TDHF. Further work to confirm these results and to extend the range of observable inelasticities is in progress.

Several long-term projects have either been completed or launched in the past year. Our computing facility is now first class. The PDP $11 / 60$ data collection system is in routine use and the singles and coincidence programs have been extensively developed. This new system has made possible experiments which were not feasible on our old SDS system--one example being the search for E2 transitions between the hypothesized molecular states in ${ }^{12} \mathrm{C}+{ }^{12} \mathrm{C}$ which required the recording of 11 parameter data. The VAX $11 / 780$ system has become sich an excellent analysis computer it is hard to see how we survived without it. Design work on the "momentum filter". has been completed and bids will be opened shortly. DOE, in recognition of the NUSAC recommendation for the $18 \mathrm{MeV}$ tandem has provided sufficient funds to upgrade the "momentum filter" to a mass energy product of $44 \mathrm{MeV}-\mathrm{AMU}$.

We have requested that DOE fund a high-intensity (colliding beam) polarized ion source in FY 81: If this proposal is recelved favorably we can look forward to having a very powerful and precise tool for radiative capture, parity violation and reaction studies.

We continue to welcome applications from scientists at other institutions who may wish to use any of our facilities. At present outside users are active on both the tandem anil the cyclotron. The sallent characteristics of these machines are listed on the following page. Anyone interested in using the facilities should contact a potential collaborator from the University of Washington or Dr. William Weitkamp, "Technical Director, Nuclear Physics Laboratory GL-10, University of Washington, Seattle, WA 98195 or telephone (206) $5.43-4080$. 
Let me close by reminding the reader that the articles in this report describe work in progress and are not to be regarded as publications nor quoted without permission of the investigators. The names of the investigators on each article have been listed alphabetically but where appropriate the name of the person primarily responsible for the report has been underlined.

Gre o Odelsuger

Eric G. Adelberger

Editor; 1980 Annual Report 
I. Programmable NMR B Field Regulator with IEEE Interface

$\begin{array}{ll}\text { J. RF Phase Shift Electronics. } & 37\end{array}$

K. H-Atom Signa1 Normalizer

3. NUCLEAR STRUCTURE AND REACTIONS

3.1 Gamow-Teller Giant Resonances in the $\beta^{+}$ Decay of $\mathrm{T}=\mathrm{T}_{3}=3 / 2$ Nuclei

A. Introduction

B. Spectroscopy of ${ }^{29} \mathrm{P}$ and the Giant Gamow-Teller Resonance in the $\beta^{+}$Decay of ${ }^{29} S$

C. $\mathrm{J}^{\pi}$ Assignments in ${ }^{25} \mathrm{Al}$ and the Giant Gamow-Teller Resonance in the $\mathrm{B}^{+}$Decay of ${ }^{25} \mathrm{Si}$

$3.2 \beta-\gamma$ Circular Polarization Correlation in the $\mathrm{B}^{+}$Decay of ${ }^{24} \mathrm{Al}$

3.3 Elastic and Inelastic Polarized Proton Scattering

Via Isobaric Analog Resonances in $207_{\mathrm{Bi}}$ and $209_{\mathrm{Bi}}$

3.4 Low Lying Vibrational states in $64 \mathrm{Zn}$

3.5 Depolarization in the Inelastic Scattering

of Protons from Copper

3.6 Allalyzing Puwer in the Continuum

Portions of Particle Spectra

3.7 Higher $\mathrm{T}=3 / 2$ states in ${ }^{29} \mathrm{P}$ and ${ }^{25} \mathrm{Al} \quad 64$

A. The Second Excited $\mathrm{T}=3 / 2$ state in ${ }^{25} \mathrm{Al}$. 65

B. The second Excited $\mathrm{T}=3 / 2$ state in ${ }^{29} \mathrm{Al} \quad 70$

3.8. Gamma Ray spectrum of llie $\beta^{+}$Deciay of ${ }^{24} \mathrm{Al} \quad 71$

4. RADIATIVE CAPTURE

4.1 The ${ }^{12} \mathrm{C}\left(\mathrm{p}, \gamma_{\mathrm{o}}\right)^{13} \mathrm{~N}$ Reaction 
1. ASTROPHYSICS AND COSMOLOGY

1.1 Nucleosynthesis of ${ }^{26} \mathrm{Al}$

1.2 Half-Lives of ${ }^{176} \mathrm{Lu}$ and ${ }^{180_{\mathrm{Ta}}}$. 2

1.3 Relative Yields of ${ }^{180} \mathrm{Ta}^{\mathrm{g}, \mathrm{m}}$ From the ${ }^{180} \mathrm{Hf}(\mathrm{p}, \mathrm{n})$ Reaction

1.4 Liquid Xenon Gamma Ray Detectors · 3

1.5 Aboorber-Theory Experiment . . 5

2. FUNDAMENTAL SYMMETRIES

2.1 An Improved Test of Nucleon Charge Conservation 7

2.2 Parity Mixing in the $2.8 \mathrm{MeV}$ Doublet of ${ }^{21} \mathrm{Ne} \quad 7$

2.3 Parity Mixing of the $2 \mathrm{~s}_{1 / 2}$ and $2 \mathrm{p}_{1 / 2}$ States .

A. Introduction 10

B. General Principles of the Mark II Experiment ' 11

C. Analysis of Systematic Errors in the Mark II Scheme 14

D. Considerations Involving a Possible Hydrogen Atom Parity Mixing Experiment in Zero Magnetic Field 20

E. H-Atom Atomic Beam Studies 26

F. Measurement of the Efficiency of a Lymall- $\alpha$ Delection Syatem for the Hydrogen Parity Experiment

G. A New Solenoid' for the Hydrogen Atom Experiment . 31

H. Design and Testing of Protocype RF

Cavities for the New Apparatus 
A. Unique Determination of the Amplitude and

Phase for the Population of the Giant-Dipole

Resonance in the Reaction ${ }^{12} \mathrm{C}\left(\mathrm{p}_{\mathrm{pol}}, \gamma_{\mathrm{o}}\right){ }^{13} \mathrm{~N}$

B. Measurement of $\sigma\left(90^{\circ}\right)$ and $A_{y}\left(90^{\circ}\right)$ for the

${ }^{12} \mathrm{C}\left(\mathrm{p}, \gamma_{\mathrm{o}}\right)$ Reaction From $\mathrm{E}_{\mathrm{p}}=9-14 \mathrm{MeV}$

78

4.2 Further Studies of $M 1$ and E2 Resonances in

the ${ }^{15_{N}}\left(\mathrm{p}, \gamma_{\mathrm{O}}\right)^{16} \mathrm{O}$ Reaction

80

4.3 Gamma Decay of the $\mathrm{E}_{\mathrm{x}}=10.321 \cdot \mathrm{MeV} \mathrm{1^{+ }}$,

$\mathrm{T}=1$ Level in ${ }^{40} \mathrm{Ca}$

82

4.4 Proton Capture to Excited States in ${ }^{16} 0$

A. M1 $\gamma$-Decay of the $4^{-} \cdot T=1$ Particle-Hole State

at $18.98 \mathrm{MeV}$ and the $3^{-} \mathrm{T}=1$ State at $18.03 \mathrm{MeV}$

84

B. M1 $\gamma$-Decay of the $1^{+} 16.22$ and $17.14 \mathrm{~T}=1$ States

to the Excited $0^{+}(6.05 \mathrm{MeV})$ Final state in $16_{0}$

4.5 The ${ }^{54} \mathrm{Fe}\left(\alpha, \gamma_{0}\right){ }^{58} \mathrm{Ni}$ Reaction 95

A. Introduction and Motivation 95

B. Experimental Methods and Results 95

C. Comparison with Other Experiments 99

5 MEDIUM ENERGY

100

5.1 Continuum Structure Studies and Total Inelastic

Crosg Scctiono for Low Energy $\pi^{+}$Nur.leus Scattering

5.2 Photoneutron Multiplicities at Medium Energies

6. HEAVY ION REACTIONS

6.1 Angular Momentum Dependent Leve1 Density

Limitations to Fusion

6.2 Search for $\gamma$-rays from the Quasi-molecular

$12 \mathrm{C}+12 \mathrm{C}$ system

$6.3{ }^{12} \mathrm{C}+{ }^{28}$ Si Fusion Cross Section

6.4 Search for Instantaneous Fission in the $208 \mathrm{~Pb}+{ }^{238} \mathrm{U}$ System 
6.5 Measurement of the Non-Fusion Yield in $16_{0}+16_{0}$ at $E_{\mathrm{cm}}=34 \mathrm{MeV}$

6.6 Fusion Excitation Function for ${ }^{10} \mathrm{~B}+{ }^{14} \mathrm{~N}$

$6.7{ }^{26} \mathrm{Al}\left({ }^{16} \mathrm{O},{ }^{12} \mathrm{C} \alpha\right){ }^{27} \mathrm{Al}$ Coincidence study at. $65 \mathrm{MeV}$

A. Introduction

B. Break Up Events from ${ }^{16} \cdot 0 *$

C. Equilibrium Contribution

D. Pre-equilibrium Contribution

E. Summary

6.8 Deviation from Rutherford Scattering for Heavy Ions at Energies Far Below the Coulomb Barrier

6.9 K-Shell Ionization in Heavy-Ion Collisions

6.10 Elastic Scattering of Light Heavy Ions

6.11 Total Reaction Cross Section Measurements at 35 to $200 \mathrm{MeV} / \mathrm{A}$

6.12 The Elastic Scattering Cross Section of $160+40 \mathrm{Ca}$ and the Proximity Potential

6.13 Non-Resonant Behavior of the Yield of the ${ }^{28} \mathrm{Si} 2^{+}$

7. RESEARCH BY OUTSIDE USERS

7.1 Alpha-N Yield Neutron Measurements of Importance to Reactors

7.2 Fast Neutron Beam Radiation Therapy Clinical l'rogram

7.3 Total Body Calcium by Neutron Activation

$7.4{ }^{81} \mathrm{Kr}^{\mathrm{m}}$ Production for Respiratory Physiology

7.5 Cyclotron Production of ${ }^{105} \mathrm{Ag}$

7.6 Light Ion Irradiation Creep 
7.7 A Comparison of the Therapeutic Effectiveness of BCNU Administered Concurrently with Neutron or Photon Exposures and with BCNU Administered One Day Following Neutron or Photon Exposures to a Rat Brain Tumor Model

7.8 Calibration of the Proton Registration Properties of . CR-39 Polymer

7.9 Measurement of Calcium Target Contamination

7.10 Pulsed Radioluminescence Studies

8. ACCELERATORS AND ION SOURCES

8.1 Accelerator Radiochronology

8.2 Van de Graaff Accelerator Operations and Development

8.3 Cyclotron Operations and Development

8.4 Polarized Ion Source Developments

8.5 . Sputter Ion Source Development .

8.6 Low Energy Optics Studies

8.7 Gamma Radiation Monitor System

8.8 UNIS Ion Source Improvements

9. INSTRUMENTATION AND EXPERIMENTAL TECHNIQUES

9.1 Development of a Large Area Position

Sensitive Proportional Telescope

9.2 Development of Cracked Ethylene Stripper

Foils for the Tandem

9.3 Improvements to the Rabbit Target Transport System

172

9.4 Dual Carbon Foil Time-of-Flight System

174

9.5 Th-228 Alpha Source Generator System

9.6 Design of a Large-Solid-Angle Isochronous

Momentum Filter 
9.7 Tandem Energy Controller System 180

9.8 Design and Construction of Electronic Equipment . 183

9.9 Target Preparation $\quad 184$

9.10 Compton Polarimeter Switching Bridge
Using High Power FETS

9.11 A Polarimeter for Measuring Proton
Beam Polarization

9.12 Resistive Film Position Sensitive Detector $\quad 187$

9.13 Refrigeration Unit for Chamber Cold Traps $\quad 188$

$\begin{array}{lr}\text { 10. COMPUTERS AND COMPUTING } & 189\end{array}$

10.1 CAMAC-based Data Acquisition System

for the PDP $11 / 60 \quad 189$

10.2 Microprocessor Based IEEE 488 Bus

Interface for Event Scalar 190

10.3 Progress on Singles Data Acquisition $\quad 191$

10.4 Progress in Multiparameter Acquisition 193

10.5 VAX 11/780 off-Line Computer System 194

1Ū.6 'Three-Body Trajectory Calculation 195

A. Description of the Present

Three Body Calculation $\quad 195$

B. Simulation Using Monte Carlo Method 197

C. Results of Calculations 198

D. Conclusion 202

10.7 Calculation of Multiple Scattering Corrections Using a Monte Carlo Method 203

10.8 Evaluation of Finite-Geometry Corrections in Depolarization Measurements 
10.9 Software for Analyzing Depolarization Data

10.10 A Text-Processing. System for the Preparation of Scientific Documents with the VAX Computer System and the QUME. Terminal

10.11 A Computer Program to Analyze Experiment 191 Data from LAMPF

10.12 ADJJ--A Program to Calculate Angular Diștribution Coefficients for (particle, $\gamma$ ) Reactions

11 APPENDIX

11.1 Nuclear Physics Laboratory Personnel

11.2 Ph.D. Degrees Granted, Academic Year 1979-80

11.3 List of Publications 


\section{ASTROPHYSICS · AND COSMOLOGY}

1. Nucleosynthesis of $26 \mathrm{Al}$

T.E. Chupp, K.T. Lesko, E.B. Norman, and P. Schwalbach

$26 \mathrm{Mg} / 24 \mathrm{Mg}$ isotopic abundance excesses that are correlated with the $\mathrm{Al} / \mathrm{Mg}$ elemental abundance ratios have been observed in inclusions from the Allende meteorite. This has been interpreted to indicate that ${ }^{26} \mathrm{Al}$ was present at the time of formation of the inclusions and later decayed in situ to produce the observed $26_{\mathrm{Mg}}$ excess. 1 Depending on the conditions at which the nucleosynthesis of $26 \mathrm{~A} 1$ occurs, several different nuclear reactions may play significant roles. It is believed that in stars the $25 \mathrm{Mg}(\mathrm{p}, \gamma)$ reaction is the major production mechanisn. Our analysis of this reaction was disçussed in last year 's annual report.2 In other astrophysical environments, the $26_{\mathrm{Mg}}(\mathrm{p}, \mathrm{n})$ and/or $23_{\mathrm{Na}(\alpha, n)}$ reactions may also be important production mechanisms. We have, therefore, measured the ${ }^{26} \mathrm{Al}$ production cross sections from the $26_{\mathrm{Mg}}(\mathrm{p}, \mathrm{n})$ reaction and have begun a study of the ${ }^{23} \mathrm{Na}(\alpha, n)$ reaction.

The ${ }^{26} \mathrm{~A} 1\left[5^{+}\right]$ground-state, ${ }^{26} \mathrm{~A} 1 \mathrm{~g}$, has a half-1ife of $7.2 \times 10^{5}$ yrs. However, the first excited state, $26 \mathrm{Al}^{\mathrm{m}}$, is a $0^{+}$level which $\beta^{+}$decays to $26 \mathrm{Mg}$ with a half-life of 6.3 seconds and does not decay to the $26 \mathrm{Al}$ ground-state. Thus any $26 \mathrm{Al}$ formed in this isomeric level will not survive long enough to become incorporated into a meteoritic inclusion as ${ }^{26} \mathrm{Al}$.

To distinguish between yields to the ground-state and isomer, we have studied $\gamma$-ray transitions in ${ }^{26} \mathrm{Al}$ that occur following the neutron evaporation from the $(p, n)$ and $(\alpha, n)$ reactions. We have measured -ray cross sections from threshold to $\mathrm{E}_{\mathrm{p}}=14 \mathrm{MeV}\left(\mathrm{E}_{\mathrm{bx}}=26 \mathrm{MeV}\right)$ using a $50 \mathrm{~cm}^{3}$ coaxial $\mathrm{Ge}$ (Li) detector. By summing the yields of known ground-state (isomer) transitions, we obtain lower limits to the total ground-state (isomer) yields. This technique is obviously insensitive to direct neutron evaporation to the ground-state or isomer. However, in the case of the $(p, n)$ reaction, cross sections for these processes have been measured over a limited energy range. 3

Figure $1.1-1$ shows the results of our study of the $26 \mathrm{Mg}(\mathrm{p}, \mathrm{n})$ reaction. The closed circles are our ground-state $\gamma$-ray cross sections only, while the open squares are the sum of our ground-state $\gamma$-ray cross sections and the cross sections for direct evaporation to the ground state as measured by Wong et al. ${ }^{3}$ For the energies where the data overlap, our results are in reasonable agreement with the ${ }^{26} \mathrm{Al} g$ cross section measurements of Furukawa et a1. ${ }^{4}$

Analysis of the $\gamma$-ray data from the $23 \mathrm{Na}(\alpha, n)$ reaction is currently in progress. To obtain the total ground-state yield from this reaction we plan two further experiments. We will measure the total neutron yield from this reaction using a neutron detector, and we will measure the total isomer yield using a radioactivity technique. 


\section{$\underline{\text { References }}$}

1. T. Lee, D.A. Papanstassiou, and G.J. Wasserburg, Astrophys. J. 211, L107 (1977).

2. Nuclear Physics Laboratory Annual Report, University of Washington (1979), p. 1 .

3. C. Wong, J.D. Anderson, J.W. McClure, and B. Pohl, Phys. Rev. 156, 1266 (1967).

4. M. Furukawa, K. Shizuri, K. Kamura, K. Sakamoto, and S. Tanaka, Nucl. Phys. A174, 539 (1971).

Fig. 1.1-1: Cross sections for the production of $26 \mathrm{AIg}$ from the $26 \mathrm{Mg}(\mathrm{p}, \mathrm{n})$ reaction. Closed circles represent only groundstate $\gamma$-ray cross sections. Open squares are the sum of our ground-otate $\gamma$ ray cross sections and the cross sections for direct neutron evaporation to the ground state as measured by Wong et al. 3

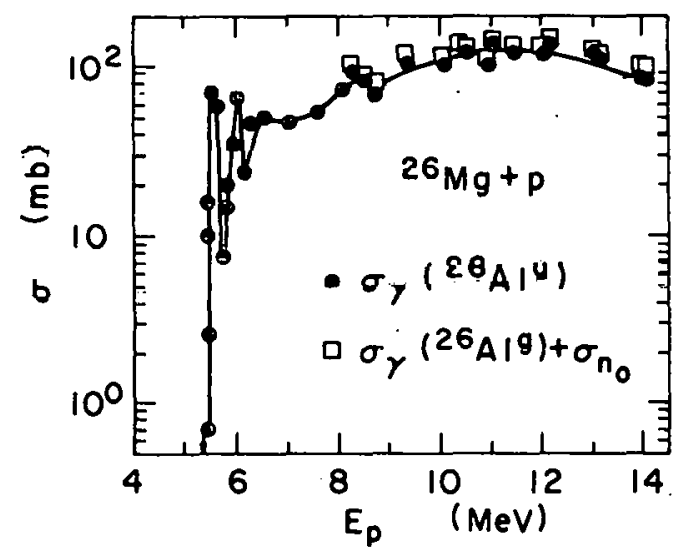

2. Half-Lives of $176_{\mathrm{Lu}}$ and $180_{\mathrm{Ta}}$

\section{E.B. Norman}

The analysis of the ${ }^{176} \mathrm{Lu}^{\prime}$ half-life experiment described in last year's annual report has been completed. The final value for the ${ }^{176} \mathrm{Lu}$ half-1ife as determined in this experiment is $t_{1 / 2}=(4.08 \pm 0.24) \times 10^{10}$ years. A paper based on this experiment has been published. 2

Another low-level counting experiment has begun in an attempt to measure the half-11fe of $180_{\mathrm{Ta}}$. A $25 \mathrm{mg}$ sample enriched to $5.1 \%$ in $180_{\mathrm{Ta}}$ has been borrowed from Oak Ridge National Laboratory for this experiment. The sample has been counted using a $\mathrm{Ge}(\mathrm{Li})$ detector, and in a spparate run, the sauple was countcd using (wo Ge(Li) detectors in coincidence. To date, no eyidence of ${ }^{180} \mathrm{Ta}$ decay has been observed and a lower limit of $t_{1 / 2}>2 \times 10^{13}$ years has been established. Further work is planned to raise the $\gamma$-ray detection efficiency and to lower the level of background counts.

\section{Refercnces}

1. Nuclear Physics Laboratory Annual Report, University of Washington (1979) p. 2 .

2. E.B. Norman, Phys. Rev. C21, 1109 (1980). 
3. Relative Yields of $180_{\mathrm{Ta}}$, m From the ${ }^{180_{\mathrm{Hf}}(\mathrm{p}, \mathrm{n}) \text { Reaction }}$

P.J. Grant, * E.B. Norman, and T.R. Renner ${ }^{+}$

${ }^{180} \mathrm{Ta}$ is an interesting odd-odd nucleus from both astrophysical and nuclear physics viewpoints. It cannot be synthesized by the conventional slow- or rapid-neutron capture processes nor by the p-process. One of the proposed production reactions is the $180 \mathrm{Hf}(\mathrm{p}, \mathrm{n})$ reaction. We have therefore continued a study of this reaction that was begun at Argonne National Laboratory.

${ }^{180} \mathrm{Ta}$ is known to have a $1^{+}$state that electron-capture decays to $180_{\mathrm{Hf}}$ and $\beta^{-}$decays to $18 q_{\mathrm{W}}$ with a half-life of 8.1 hours, but does not decay to the long-lived ${ }^{180} \mathrm{Ta}$ level. As described in Section 1.2 of this report, the half-life of the long-lived $180 \mathrm{Ta}$ is $>2 \times 10^{13}$ years. To distinguish between the yields to these two levels we have performed two separate experiments. . We have measured the cross sections for production of the 8.1 hour state by bombarding either natural Hf or enriched $180_{\mathrm{Hf}}$ targets with a proton beam. The targets were then removed to an off-line location and were counted using a $\mathrm{Ge}(\mathrm{Li})$ detector. In a separate experiment, a thick $180_{\mathrm{Hf}}$ target was bombarded with protons and the total neutron yield from the $(p, n)$ reaction was measured using a spherical long-counter. ${ }^{1}$ The yield of the long-lived ${ }^{180} \mathrm{Ta}$ state will be determined by subtracting the yield of the 8.1 hour level from the total $(p, n)$ yield. Analysis of the data from these experiments is currently in progress.

\section{$\underline{\text { References }}$}

* Department of Nuclear Engineering, University of Washington.

+ Department of Physics, S.U.N.Y. at Stony Brook.

1. Nuclear Physics Laboratory Annual Report, University of Washington (1979) p. 131.

4. Liquid Xenon Gamma Ray Detectors

J.G. Cramer, C.R. Gruhn,* and R. Loveman

In the past year we have completed the investigation of the 11near polarization sensitivity to $X$-rays and gamma rays of liquid xenon and liquid argon filled ion chambers. The effect investigated is based on the fact that the electrons ejected by a photoelectric event of an X-ray or gamma ray tend to lie in the plane defined by the electric field of the photon and its forward momentum vector, i.e., the plane of linear polarization, and thus form a "sheet" of ionization in the detection volume. If this sheet is oriented parallel to the collection plate the resulting pulse will have a short rise-time, while if the sheet is perpendicular to the collection electrode a slow-rising pulse will result. Thus the pulse rise-time can, in principle, be used to tag the linear polarization direction and/or the forward momentum direction of the detected $X$-ray or gamma ray. 
Monte Carlo calculations have been performed to investigate the strength o: this effect and its effective analyzing power for linearly polarized photons. These Monte Carlo calculations indicate that while the expected linear polarization sensitivity is present, the effect is probably too small at the energies considered ( 30 to $80 \mathrm{keV}$ ) to make the method feasible in a liquid argon filled detector when the noise contribution of the associated electronics is considered. On the other hand, the technique may be feasible at somewhat higher energies (which were not considered because of the complications of the Compton effect) and particularly when a gas-filled detector employing gas-multiplication amplification is employed. It should also be pointed out that the same calculations indicate that the technique can successfully be employed to make such a detector direction sensitive (at the level of a few degrees) at higher energies. A paper on this was presented at the Knoxville DNP Divisional. Meeting last Fall, 1 and a publication baced on these results is in preparation.

In the past year we have, in collaboration with Dr. C.R. Gruhn of LBL, conducted an investigation of the effert of "doping" a liyuld argon tilled ionizallun chamber with a small admixture of xenon. The expectation was that this might enhance the ion.production of charged particles by promoting energy transport through ultraviolet production. However, our measurements indicate that this is not the case and that xenon doping reduces the net ionization. This result may arise from possible impurities in the xenon introduced into the system, and in any case is not understood at present. When xenon-purification techniques are better in hand these measurements may be repeated.

We have also collaborated with Dr. Gruhn in the investigation of ionic recombination in liquid argon of the ionization of energetic heavy ions. The preliminary results of this investigation indicate that the recombination depends linearly on the range (as opposed to the energy or velocity) of the ions studied.

1 The measurements of gamma ray line shapes in liquid-xenon filled ion chambers under good measurement conditions has been slowed by the high cost of xenon gas and the unavailability of funds at the University of Washington to purchase it in sufficient quantity. However, this problem has been solved for the moment by the decision of LBL to commit significant funding to this project. For this reason, the focus of our efforts has shifted to $L B L$ and one of us (Loveman) is in residence at LBL working full time on this project. The xenon-detector cryostat has been assembled, and the xenon purchased. The initial efforts with this system have been aimed at obtaining the best possible energy resolution while detecting conversion electrons with the apparatus filled with liquid argon. Recent results of such measuremencs are shown in Fig. 1.4-1. Conversion electrons with an energy of $976 \mathrm{keV}$ from a $207_{\mathrm{Bi}}$ source are detected in a liquid argon filled ion chamber. The overall resolution of the peak is $40 \mathrm{keV}$, and the electronic (pulser) noise of the system is about $28 \mathrm{keV}$. This implies an intrinsic resolution for the detector of about $24 \mathrm{keV}$. This represents the best energy resolution which has ever been obtained with such a detector. 
In the coming year, we expect to pursue our investigations of argon and xenon filled ion chambers and in particular to make a set of definitive measurements of the gamma ray detection characteristics of xenon-filled detectors. The development of a liquid-xenon filled detector with an active volume on the order of 1 liter is one of the goals of this project.

\section{References}

* Lawrence Berkeley Laboratory, Berkeley, California.

1. R.A. Loveman and J.G. Cramer, Bull. Am. Phys. Soc. 24, 823 (1979).

Fig. 1.4-1: Pulse height spectrum of conversion electrons from $207 \mathrm{Bi}$ detected in liquid argon filled ion chamber. The peaks labelled $e_{\mathrm{K}} \quad \mathrm{e}_{\mathrm{L}}$ correspond to $\mathrm{K}$ and $\mathrm{L}$ convers ion electrons, respectively. The broad peaks labelled C.E. are the compton edges of the corresponding gamma rays. The FWHM of the $976 \mathrm{keV}$ electron line is $40 \mathrm{keV}$ and the FWHM of the pulser peak is $28 \mathrm{keV}$.

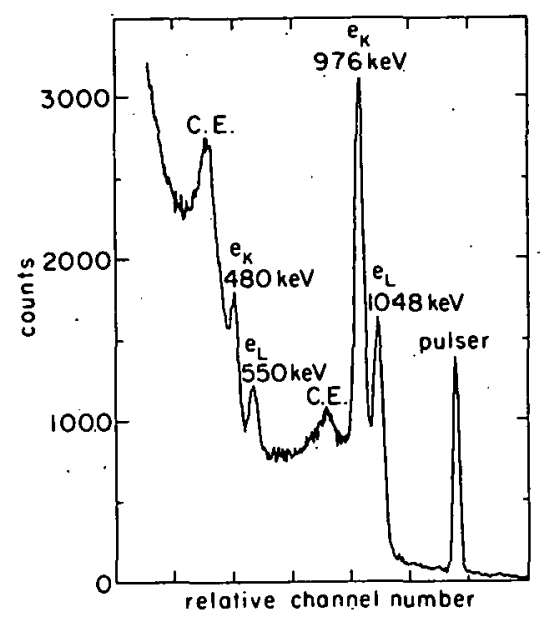

\section{Absorber-Theory Experiment}

J.G. Cramer, D. Leach, and A. Seamster

We are presently performing an experiment to look for possible spatial anisotropies in the emission of neutrinos which are predicted by Wheeler-Feynman absorber theory as it applies to the emission of neutrinos, for the circumstance where there is a deficiency of future absorption of neutrinos. This measurement is to some extent the neutrino analog of the absorber theory experiments performed by Partridge ${ }^{2}$ and by Schmidt and Newman, ${ }^{3}$ both of which have enployed about $10 \mathrm{GHz}$ microwaves in a search for emission anisotropies or other advanced-wave effects. The measurement which is in progress is a search for a spatial anisotropy in the emission of neutrinos, as reflected by a somewhat smaller anisotropy in the emission of angle-correlated beta particles in a pure Ganow-Teller beta decay.

The experiment which has been designed for these anisotropy measurements employs ${ }^{106} \mathrm{Ru}$, a pure Gamow-Teller beta source as the emitter of neutrinos, while recording the direction-correlated beta particles from the radioactive decay with symmetrically placed scintillation counters. Since last year a new 
high precision sidereal clock has been incorporated in the data recording apparatus. Over a thousand asymmetry spectra have been measured at half hour intervals and recorded on magnetic tape. Analysis of these data has shown systematic variations in the relative counting rates of the two scintillation counters having a 24 hour period and properties indicating a systematic error. This "background" error would correspond to an asymnetry of about $0.05 \%$. It is presumably due to effects arising from daily room temperature variations, powerline fluctuations, commercial radio broadcasts, etc. The experimental setup has been moved from the Nuclear Physics Laboratory to Physics Hall where the ambient environment can be more carefully controlled. This seems to have eliminated some, but not all, of the systematic error problems.

Our principal problem in the coming year will. be to eliminate the serious systematic errors mentioned above, so that an asymmetry on the order of $0.01 \%$ or less could be observed if it were present. This is abnut the level at which optimistic models might predict an effect. Our first steps in this direction will he more careful attention to power isolation, control of ambient temperature, and electricai shielding of the apparatus.

\section{References}

1. J.A. Wheeler and R.P. Feynman, Rev: Mod. Phys. 17, 157 (1945); ibid. 21,425 (1949).

2. R.B. Partridge, Nature 244, 263 (1973).

3. J. Schmidt and R. Newman, Bull. Am. Phys. Soc. 25, 581 (1980). 


\section{FUNDAMENTAL SYMMETRIES}

\section{An Improved Test of Nucleon Charge Conservation}

\section{E.B. Norman and A.G. Seamster}

As described in last year's annual report ${ }^{-1}$ we have searched for charge-non-conserving ( CNC) decays of the type

$$
\mathrm{n} \rightarrow \mathrm{p}+\text { neutrals }
$$

using the nuclei ${ }^{87} \mathrm{Rb}$ and ${ }^{87} \mathrm{Sr}$. A $400 \mathrm{gm}$ sample of $\mathrm{Rb}_{2} \mathrm{CO}_{3}$ was dissolved in 200 $\mathrm{ml}$ of $\mathrm{H}_{2} \mathrm{O}$. A known amount of $\mathrm{Sr}^{++}$carrier was then added to the solution. The non-radioactive $\mathrm{Sr}$ and any $87 \mathrm{Sr}^{\mathrm{m}}$ (produced by the CNC decay of $87 \mathrm{Rb}$ ) precipitated as $\mathrm{SrCO}_{3}$ and were filtered out of solution. The separated $\mathrm{SrCO}_{3}$ was then placed on the front of a well-shielded Ge(Li) detector and a search was made for the $388.4 \mathrm{keV} \gamma$-ray that is emitted by the decay of the 2.8 hour ${ }^{87} \mathrm{Sr}^{\mathrm{m}}$.

This procedure was repeated five times during a five-day period. No excess of counts above background was observed in the vicinity of $388.4 \mathrm{keV}$. From this experiment a lower limit of $t_{1 / 2}>1.9 \times 1018$ years has been established for this charge-non-conserving decay. This represents a one-hundred fold improvement over the only other measurement of its type. ${ }^{2}$ A paper based on this experiment has been published. 3

\section{References}

1. Nuclear Physics Laboratory Annual Report, University of Washington (1979), p. 9.

2. A.W. Sunyar and M. Goldhaber, Phys. Rev. 120,871 (1960).

3. E.B. Norman and A.G. Seamster, Phys. Rev. Lett. 43, 1226 (1979).

2. Parity Mixing in the $2.8 \mathrm{MeV}$ Doublet of $21_{\mathrm{Ne}}$

E.G. Adelberger, A.B. McDonald*, H.E. Swanson, and R.D. VonLintig

The parity mixing in the $2.8 \mathrm{MeV} \mathrm{J}=1 / 2$ doublet of the odd-neutron nucleus $21_{\mathrm{Ne}}$ has become an interesting system for quantitative measurement of the relative magnitudes of the $\triangle I=0$ and $\Delta I=1$ parity nonconserving $N-N$ forces. Because of the very smali energy splitting between the $J=1 / 2^{+}$and $J=1 / 2^{-}$ states $(\triangle E=7.6 \mathrm{keV})$, and the combination of a highly retarded El transition $\left(\tau_{E 1} \sim 696 \pm 51 \mathrm{ps}\right)$ and a fast $M 1$ transition ( $\left.\tau_{M 1} 6.2 \pm 1.0 \mathrm{fs}\right)^{1}$ the circlar polarization of the $2.789 \mathrm{MeV} \gamma$-ray provides an extremely sensitive probe of the parity violating matrix element. Recent calculations of the $21_{\mathrm{Ne}}$ 
wavefunctions 2,3 combined with $S U(6)_{\mathrm{w}}$ calculations of the parity nonconserving $\mathrm{N}-\mathrm{N}$ amplitudes have been very successful in accounting for the unexpectedly small circular polarization $\left[\mathrm{P}_{\gamma}=(0.14 \pm 0.29) \times 10^{-2}\right]$ observed in the measurement presented in last year's Annual Report. ${ }^{-}$The small effect arises from a near cancellation of the $\triangle I=0$ and $\Delta I=1$ matrix elements which is consistent with a neutral current contribution described by the Weinberg-Salam model.

Because of the inherent importance of the ${ }^{21} \mathrm{Ne}$ system we have concentrated our efforts on improving the experimental results in $21_{\mathrm{Ne}}$. During the past year we have designed an improved version of the circular polarization experiment (Mark II) and remeasured the efficiency of the Mark I circular polarimeters.

Our Mark I experiment was count-rate limited. The Mark II experiment should increase our counting rate by roughly an order-of-magnitude. This increase results from using four polarimeters with 5 " $\times 6^{\prime \prime}$ NaI detectors instead of two polarimeterswith 3" $\times 3^{\prime \prime}$ detectors. The use of 4 rather than 2 polarimeters also reduces systematic errors due to beam "steering". in the . stray fields from the polarimeter. The polarimeters are being constructed at Chalk River and are similar in concept to the Mark I version. The $5^{\prime \prime} \times 6^{\prime \prime}$ detectors have been delivered to Chalk River, and have been tested at $7.5 \%$ resolution at $1333 \mathrm{keV}$. A new constant-current switching circuit for driving the polarimeters (described in Sec. 9.10 of this report) has been constructed in Seattle. We plan to begin data taking this summer.

Fig. 2.2-1: Spectrum of $\gamma$-rays from ${ }^{24} \mathrm{Na}$ transmitted through two Compton polarimeters.

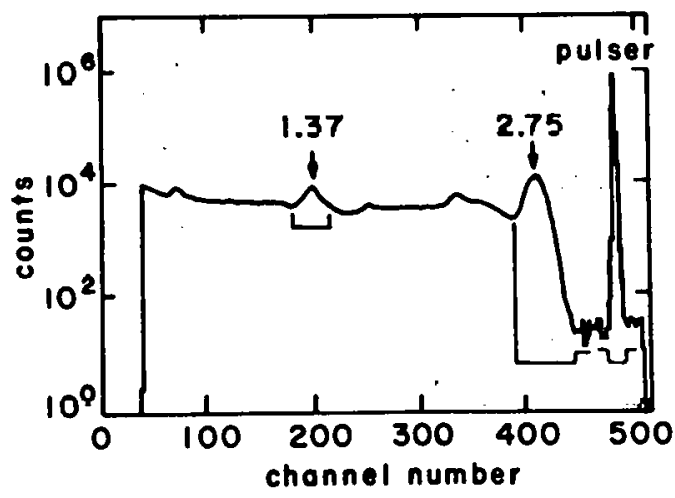


We have remeasured the efficiency of our Mark I polarimeters at $E_{y}=2.754$ $\mathrm{MeV}$ using $24 \mathrm{Na}$ sources produced by $23 \mathrm{Na}(\mathrm{n}, \gamma)$ at the University of Washington reactor. The technique was described in last year's Annual Report. 4 However the data shown in last year's report displayed non-statistical deviations. We have redone the efficiency measurement with results which are now consistent with Poisson statistics.

A spectrum of $\gamma$-rays from a ${ }^{24} \mathrm{Na}$ source as transmitted by the two polarimeters in series is shown in Fig. 2.2-1. A "random" pulser was used to make small dead time corrections. The efficiencies measured with a series of ${ }^{24} \mathrm{Na}$ sources along with the average value is shown in Fig. 2.2-2. The final result

Fig. 2.2-2: Circular polarimeter efficiency measurements for a series of ${ }^{24} \mathrm{Na}$ sources.

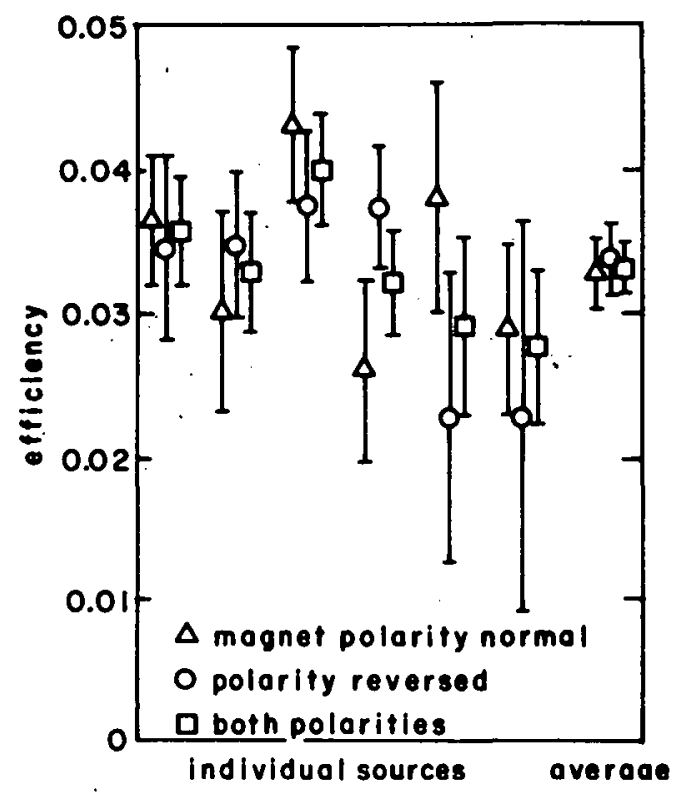

$$
\eta_{2.754}=(3.35 \pm 0.18) \times 10^{-2}
$$

is in excellent agreement with the value $72.754=(3.40 \pm 0.10) \times 10^{-2}$ obtained by scaling the efficiency measured by Lowry at Caltech 6 with a ${ }^{60}$ Co source. Combining the results of this work and Ref. 5 we find that the analyzing power at $\mathrm{E}_{\mathrm{y}}=2.789 \mathrm{MeV}$ as

$$
\eta 2.789=(3.39 \pm 0.08) \times 10^{-2}
$$

\section{References}

* Chalk River Nuclear Laboratories, Chalk River, Ontario, Canada.

1. E.K. Warburton, J.W. Olness, and C.J. Lester, Phys. Rev. C20, 619 (1979).

2. D.J. Millener et al., Phys. Rev. C18, 1878 (1978).

3. Wick Haxton, private communication, $\overline{1980}$.

4. Nuclear Physics Laboratory Annual Report, University of Washington (1979), p. 13 .

5. C.A. Barnes et al., Phys. Rev. Lett. 40, 840 (1978).

6. M.M. Lowry, $\overline{\mathrm{Ph}} \cdot \overline{\mathrm{D}}$. thesis, Caltech (1978) unpublished. 
3. Parity Mixing of the $2 \mathrm{~s}_{1 / 2}$ and $2 \mathrm{p}_{1 / 2}$ States in Hydrogen and Deuterium Atoms

A. Introduction

\section{E.G. Adelberger}

The parity nonconserving (PNC) interactions between electrons and nucleons provide a detailed probe of the neutral current weak interaction. One may, in lowest order, distinguish 4 separate PNC couplngs: $g_{A}{ }^{e} g_{V}^{p}, g_{V}^{e} g_{A}{ }^{p}, g_{A}^{e} g_{V}^{n}$, and $g_{V}{ }^{e} g_{A}{ }^{n}$ whose strengths (following Ref. 1) we denote by $C_{1} p, C_{2}{ }^{p}, C_{1}{ }^{n}$ and $\mathrm{C}_{2} \mathrm{n}$. Measurements of the parity mixing between specific hyperfine components of the $2 s_{1 / 2}$ and $2 p_{1 / 2}$ levels of hydrogen and deuterium atoms can, in principle, yield all four of the PNC coupling constants with essentially nn . unrertainty from atomic theory. Note that for deuterium we have

$$
c_{1}{ }^{d}=c_{1} p+c_{1}^{n} \quad \text { and } \quad c_{2}{ }^{d}=c_{2} p+c_{2}{ }^{n}
$$

If experiments in $H$ and $D$ achieve sufficient accuracy one can resolve some very fundamental issues. In the Weinberg-Salam (WS) model the lowest order expressions for the coupling constants are:

$$
\begin{array}{ll}
c_{1}^{p}=1 / 2\left(1-4 \sin ^{2} \theta_{N}\right) & c_{1}^{n}=-1 / 2 \\
c_{2}{ }^{p}=1 / 2 g_{A}\left(1-4 \sin ^{2} \theta_{W}\right) & c_{2}^{n}=-1 / 2 g_{A}\left(1-4 \sin ^{2} \theta_{W}\right)
\end{array}
$$

It would be clearly interesting to measure all four of these constants and check whether they agree with the WS predictions. Existing experiments in heavy atoms $^{2}, 3,4,5$ and high-energy electron scattering ${ }^{6}$ determine only certain linear combinations of the constants and significant uncertainties occur in the theoretical interpretations--especially in the heavy atoms. Perhaps even more interesting than checking the lowest order WS predictions are the higher order effects. In the WS model the coefficient $C_{2} d$ vanishes identically in lowest order. However, the radiative corrections do not vanish--in fact the second order terms are expected to contribute to $C_{2} d$ at the $0.01-0.05$ level. ${ }^{7}$ The experimental determination of $\mathrm{C}_{2} \mathrm{~d}$ - thus directly measures the weak radiative corrections in any model in which the axial weak neutral current is purely isovector! There may also be a contribution to $\mathrm{C}_{2}{ }^{\mathrm{d}}$ from induced isoscalar axial currents due to virtual ss quark excitations in the nucleon. 8

The relevant $\mathrm{N}=2$ levels of hydrogen are shown in Fig. 2.3.A-1 to establish our notation. The PNC interaction can mix the following levels: $\alpha_{n} e_{0}, \alpha_{n} f_{n}$, $\alpha_{+} e_{+}, \beta_{o} e_{o}, \beta_{o} f_{o}$, ad $\beta_{-} f_{-}$. Due to the $1 / r$ Coulomb potential the $\beta_{0}-e_{o}$ mixing in hydrogen is sensitive only to $\mathrm{C}_{2} \mathrm{p}$ and in deuterium to $\mathrm{C}_{2}$. fundanental test of electroweak interaction theory. 
Fig. 2.3.A-1: $\mathrm{n}=2, \mathrm{~J}=1 / 2$

levels of hydrogen

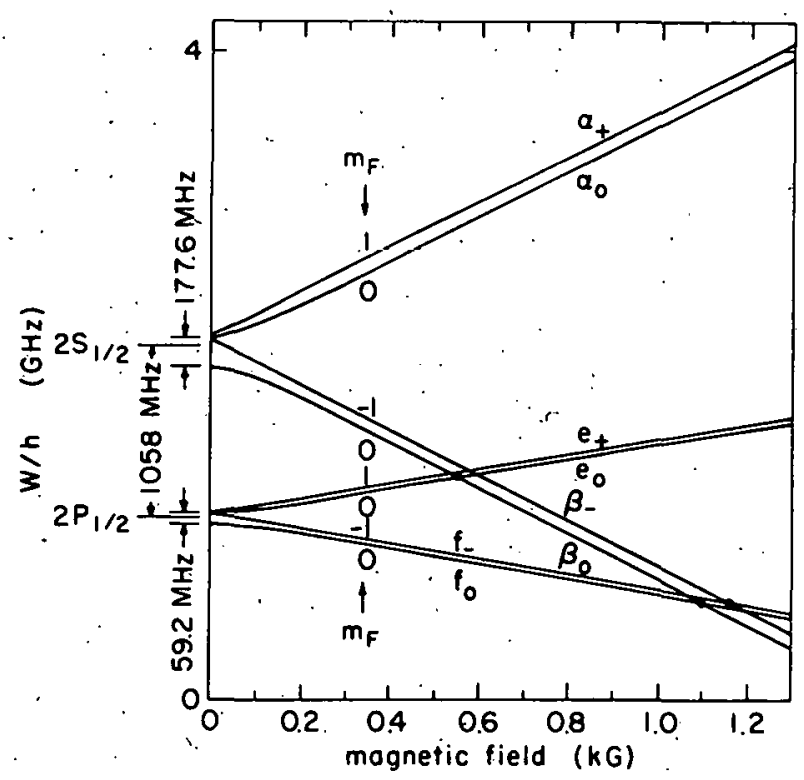

In this article we describe a new.technique (denoted Mark II) for meas,uring the PINC mixing with a sensitivity much greater than that of a method (Mark. I) we described previously. 9 . The new scheme was developed after studies of systematic errors on the Mark I apparatus showed that spurious effects were much too large to allow a meaningful measurement of $\mathrm{C}_{2}$. The Mark II scheme is such a large improvement over Mark 'i that we have abandoned the Mark I approach and are putting all, our emphasis on designing and constructing the new apparatus. The remainder of this section discus'ses the principles of the Mark II scheme, measurements made with the Mark I apparatus and progress on building the Mark II apparatus.

B. General Principles of the Mark II Experiment

E.G. Adelberger, T.E. Chupp, E.N. Fortson, D. Holmgren, M.Z. Iqbal, H.E. Swanson, and T.A.: Trainor

Several groups $9,10,11$ have proposed microwave schemes for measuring $\beta_{0}-e_{0}$ and $\beta-f$ PNC mixing at level crossings using $500 \mathrm{eV}$ beams of metastable hydrogen. Our Mark II scheme, in common with those proposed in Refs. 9 and 10 detects the PNC $\alpha \beta_{0}-e_{0}$ mixing by driving $\alpha_{0} \rightarrow \beta_{0}$ electric-dipole transitions in a uniform magnetic field of $\sim 570$ gauss near the $\beta^{-e}$ crossings. The transition proceeds predominantly via intermediate $e$ states and we detect the interference between weak interaction and Stark effect $\beta_{0}-e_{g}$ mixing. However, in the Mark. II scheme the. PNC amplitude $A_{P N C}\left(\alpha_{0} \rightarrow e_{0}{ }_{P} \rightarrow \beta_{0}\right)$ produced in one RF cavity interferes with a parity conserving amplitude ${ }_{\mathrm{A} C}\left(\alpha_{0} \vec{R}_{\mathrm{z}} \mathrm{e}_{0} \rightarrow \beta_{0}\right)$ produced in another cavity. Our signal is the change In the $\alpha_{0}-\beta_{0}$ transition rate when the relative phase of $A_{P C}$ and $A_{P N C}$ is changed by $180^{\circ}$. This can be achieved by changing the relative phase of the $R F$ in the two cavities by $180^{\circ}$, by reversing the direction of $E$, and by reversing $B$. In our notation $R=\langle e|e \vec{R} \cdot \vec{r} / 2 h| \alpha\rangle$ is 
Fig. 2.3.B-1: Schematic diagram of the experimental configuration.

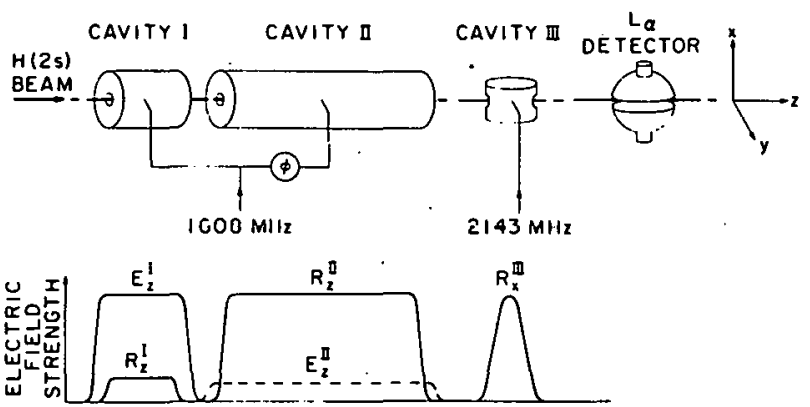

the matrix element of a microwave electric field $(\vec{R})$ oscillating at $\omega_{R F}=$ $2 \pi \cdot 1608 \cdot 10^{6} \mathrm{sec}^{=1}, \quad V_{\mathrm{PNC}}=i \tilde{V}_{\mathrm{PNC}}=\left\langle\beta_{\mathrm{O}}\left|\left(\mathrm{H}_{\mathrm{PNC}} / \hat{h}\right)\right| \mathrm{e}_{\mathrm{O}}\right\rangle=-0.16 \mathrm{iC}_{2}{ }^{\mathrm{P}} \mathrm{sec}^{-1}$ is the weak matrix element, $\dot{E}=\langle e|e \vec{E} \cdot \vec{r} / \hbar| \beta\rangle$ is the matrix element of a static electric field $(\vec{E})$, and $\hat{z}$ is the magnetic field axis. (In this paper all frequencies are angular frequencies measured in radians/sec.) At our RE frequency the unperturbed $\alpha_{0} \rightarrow \beta_{0}$ resonance occurs at 569.4 gauss. The transition rate is

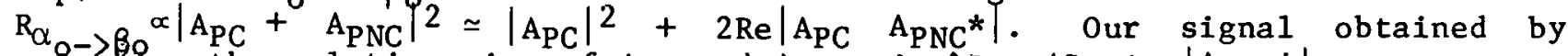
reversing the relative sign of $A_{P C}$ and $A_{P N C}$, is $\triangle R=4 R e A_{P C}\left|A_{P N C}{ }^{*}\right|$.

The experimental geometry is shown in Fig. 2.3. B-1. $500 \mathrm{eV}$ protons from a duoplasmatron, converted by charge exchange in $C s$ vapor into a beam of $H(2 s)$, enter a 570 gauss solenoid. The beam passes through a transverse, static E field to quench the $\beta$ levels: The resulting beam of atoms in the $\alpha_{+}$and $\alpha_{0}$ states then enters two successive cylindrical RF cavities oscillating coherently in the TMOlo mode. In each cavity there are RF and static $\vec{E}$ fields along $\hat{z}$. Since the RF $B$ field vanishes on the cavity axes we shall temporarily neglect Ml transitions between the 2 s states. The first, shorter, cavity drives APC. It contains weak $R F$ and strong static electric fields $\vec{R}_{z} I$ and $\vec{E}_{z} I$ with matrix elements $R I$ and $E I$, respectively. Therefore the RF quenching of the $\alpha$ states is small and the ${ }^{z}$ PNC mixing can be neglected in comparison to the Stark mixing induced by the static $\vec{E}$ field. The second, longer, cavity drives APNC. The RF in this cavity is strong $\left(z^{2}=\sqrt{\gamma / t_{2}}\right)$ and is shifted in phase with respect to that in the first cavity. The weak static $\vec{E}$. field along $\hat{z}$ (with matrix element $\left.E_{z} I I\right)$ drives a probe transition, $A_{p}\left(\alpha_{0} \cdot R_{z} e_{z} \beta_{o}\right)$. The purpose of $A_{p}$ will be discussed below. The beam then passes through a cavity containing a perpendicular $R F \vec{E}$ field oscillating at $2 \pi \cdot 2143 \cdot 10^{6} \mathrm{sec}^{-1}$ which depopulates the $\alpha_{+}$and $\alpha_{0}$ levels by $\alpha-f$ mixing. The remaining $\beta$ states are detected by passing the beam through a static perpendicular $\vec{E}$ field. We deternine the $\alpha_{0} \rightarrow \beta_{0}$ transition rate by detecting the Lyman radiation from this $\beta-e$ mixing.

The main features of our scheme can be understood by treating $E_{z} I, E_{z} I I$, $R_{z} I, \quad R_{z} I I$ and $V_{P N C}$ as perturbations and neglecting intermediate $f$ states. We assume $R_{z} I I \ll \gamma$. $E_{z} I \ll \gamma, E_{z} I I \ll R_{z} I I$, and $R_{z} I \ll E_{z} I$, where $\gamma$ is the $2 \mathrm{p} \rightarrow 1 \mathrm{~s}$ decay rate. Then at the $\alpha_{0} \beta_{0}$ resonance, an atom initially in the $\alpha_{0}$ state which spends times $t_{1}$ and $t_{2}$ in cavities 1 and 2 , respectively, has a probability to be in the $\beta_{0}$ state given by: 


$$
\begin{aligned}
& |\beta|^{2}=\mid-\frac{0.11 E_{z}^{I} z}{\frac{\gamma}{2}-i \omega_{B_{0} e_{0}}}\left(\frac{1-e^{-\Gamma_{1} t_{1}}}{\Gamma_{1}}\right) \\
& -\left.\frac{\left(i \tilde{V}_{\text {PNC }}+0.11 E_{z}^{I I}\right) \tilde{R}_{z}^{I I} e^{i \phi} e^{-\Gamma_{2}{ }^{t} 1}}{\frac{\gamma}{2}-i \omega_{\beta_{0}} e_{o}}\left(\frac{1-e^{-\Gamma_{2} t_{2}}}{\Gamma_{2}}\right)\right|^{2}
\end{aligned}
$$

where $\hbar \omega_{\beta_{o} e_{o}}=E_{\alpha_{0}}-E_{e_{o}}-\hbar \omega_{R F}, \hbar \omega_{e \beta}=E_{e_{o}}-E_{\beta_{o}}, \quad \hbar \delta=E_{\alpha_{o}}-E_{\beta_{o}}-\hbar \omega_{R F}$,

$$
\Gamma_{1}=\frac{\left(\begin{array}{l}
E_{z}^{I} \\
\mid
\end{array}\right)}{\frac{\gamma}{2}+i \omega_{e_{0}}}+i \delta, \quad \text { and } \quad \Gamma_{2}=\frac{\left(R_{z}^{I I}\right)^{2}}{\frac{Y}{2}-i \omega_{\alpha_{0}} e_{0}}+i \delta
$$

and we have ignored any drift space between the two cavities. The relative phase of the oscillations in the two cavities has been included by defining $R_{z} I$ to be real and setting $R_{z} I I=\tilde{R}_{z}{ }^{I I} e^{i \phi}$ where $\phi$ is the relative phase.

The phase $\phi$ is set to make the interference of APNC and APC as large as possible ( $A_{P N C}$ and $A_{P C}$ relatively real). This can be achieved by requiring the interference of $A_{P C}$ with $A_{P}$ to vanish (since $A_{P}$ and $A_{P N C}$ are 900 out of phase). The angle $\phi$ is controlled as follows. The weak static $E$ field in the second cavity is modulated at frequency $\omega_{m}$ and lock-in techniques are used to detect any component of the $\beta$ state signal which occurs at a frequency $\omega_{m}$. This component arises from $A_{P C}$, AP interference. It vanishes if $\phi$ has been set correctly and is used to control an electronic phase-shifter in a feedback loop.

$\mathrm{V}_{\mathrm{PNC}}$ can be measured by detecting the change in the counting rate of $\beta_{0} \mathrm{O}$

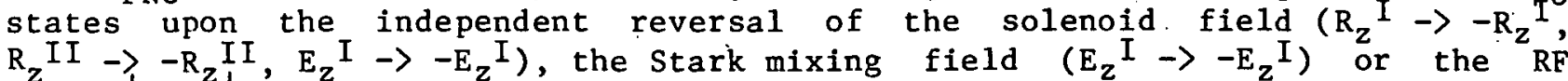
phase $\phi \rightarrow{ }^{2} \phi+180^{\circ}$. This change is

$\Delta|\beta|^{2}=4 \operatorname{Re} \frac{0.11 E_{z}^{I} R_{z}^{I}\left(i \tilde{V}_{P N C}+0.11 E_{z}^{I I}\right) \tilde{R}_{z}^{I I} e^{i \phi-\Gamma_{2} t_{1}}}{\left[\frac{\gamma}{2}-i \omega_{\beta_{o} e_{o}}\right]\left[\frac{\gamma}{2}+i \omega_{\beta_{o} e_{o}}\right]} \times \frac{1-e^{-\Gamma_{1}^{*} t_{1}}}{\Gamma_{1}^{*}} \frac{1-e^{-\Gamma_{2} t_{2}}}{\Gamma_{2}}$ 
Fig. 2.3.B-2: Calculated signal for the case where the front and rear cavities have lengths of $12 \mathrm{~cm}$ and $36 \mathrm{~cm}$, respectively. Electric fields are assumed to turn on and off abruptly and are roughly opti-. mum if there is a negligible background in the $\mathrm{L}_{\alpha}$ detectors. Lower pall: Lhe $a_{0} \beta_{0}^{\alpha}$ lesumalice yield. Upper part: the modulation of the $\alpha_{0} \beta_{0}$ yield when the relative phases of APC and $A_{P N C}$ are reversed. We assume $\mathrm{C}_{2}=0.5$.
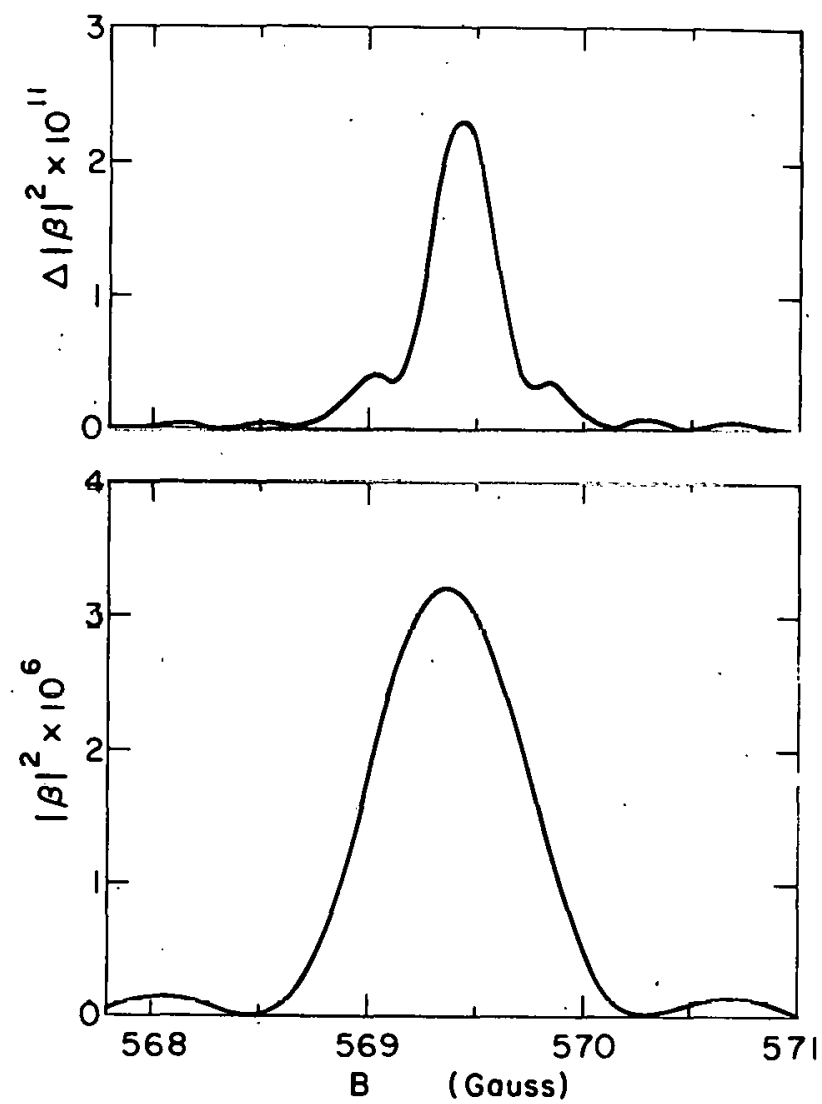

In Section 2.3.C we discuss an additional subtraction involving $\delta$.

The results of a numerical calculation modelling our experiment are displayed in Fig. 2.3.B-2. The lengths of the first and second cavities are 12 and $36 \mathrm{~cm}$, respectively. For simplicity the fields $t_{z} \vec{E}_{z} \vec{E}_{z}$, and $\vec{R}_{z}{ }$ and $\vec{R}_{z} I I$ are assumed to turn on and off abruptly and the magnitudes are rough1y optimized for the ideal case of a negligible background in the Lyman $\alpha$ detectors. Our present ion source produces a metastable beam intensity of $\sim 1$ particle $\mu$ amp in each hyperfine state into a solid angle of $2 \times 10^{-5} \mathrm{sr}$ and we have constructed a $\beta$-state detector with a total efficiency of $1 / 8$. With this intensity a statistical error in $\mathrm{C}_{2} \mathrm{p}$ of $\sim 0.16$ can be reached in one day of running time.

C. Analysis of Systematic Errors in the Mark II Scheme

E.G. Adelberger, T.E. Chupp, M.Z. Iqbal, and T.A. Trainor

The various schemes $9,10,11$ which have been suggested to study PNC effects in $H(2 s)$ are roughly equivalent as regards statistical errors since for a given metastable atom flux and counting time, the signal/noise ratio is proportional to $V_{\mathrm{PNC}} \sqrt{\mathrm{T} / \gamma}$ where $\mathrm{T}$ is the time the beam spends on the interaction region. Our scheme is a little more favorable than that of Ref. 10 since we have a larger effect ( $A_{P C}$ and $A_{P N C}$ are relatively real) and $\beta^{\prime} s$ produced by $V_{P N C}$ are not quenched by transverse $\vec{E}$ fields. 
However, the different schemes have quite different sensitivities to systematic errors. These are dominated, in decreasing order of importance, by: 1) motional $\vec{E}$ fields $\vec{E}_{m}=\vec{v} / c \times \vec{B} ; 2$ ) misalignment and non-uniformity of the applied fields; 3) stray electric fields; and 4) M1 transitions.

It is convenient to express the $\alpha_{0} \rightarrow \beta_{0}$ amplitudes as scalar products of the external fields acting on the $\mathrm{H}(2 \mathrm{~s})$ atoms. In this notation

$$
\begin{aligned}
& A_{P N C}=0.5 i \tilde{V}_{P N C} V_{o}(\vec{R} I \cdot \hat{B}) t_{2} / D_{o} \\
& A_{P}=0.055 v_{o}^{2}\left(\vec{R}^{I I} \quad \cdot \hat{B} \vec{E}^{I I} \cdot \hat{B}\right) t_{2} / D_{o}
\end{aligned}
$$

and

$$
\mathrm{A}_{\mathrm{PC}}=0.055 \mathrm{v}_{\mathrm{o}}^{2}\left(\overrightarrow{\mathrm{R}}^{\mathrm{I}} \cdot \mathrm{B} \quad \overrightarrow{\mathrm{E}}^{\mathrm{I}} \cdot \hat{\mathrm{B}}\right) / \mathrm{t}_{1} / \mathrm{D}_{\mathrm{O}}
$$

where $D_{0}=\left(\gamma / 2-i \omega_{\beta_{0}} e_{o}\right)$

One must realize that the states $\alpha_{0}$ and $\beta_{0}$ can also be connected by the amplitudes

$$
\begin{aligned}
& A_{M}=0.055 U_{0}(\vec{M} I I \cdot \hat{B}) \\
& A_{J_{1}}=-0.0275 \mathrm{~V}_{0}^{2}(\vec{R} I I \times \hat{B}) \cdot(\vec{E} I I \times \hat{B}) / t_{2} / D_{+},
\end{aligned}
$$

and

$$
\mathrm{A}_{J_{2}}=-0.0275 i \mathrm{~V}_{\mathrm{O}}^{2}\left(\overrightarrow{\mathrm{R}}^{\mathrm{II}} \times \overrightarrow{\mathrm{E}}^{\mathrm{I}} \cdot \hat{\mathrm{B}}\right) \mathrm{t}_{2} / \mathrm{D}_{+}
$$

where $\vec{M}$ is the RF magnetic field with matrix element $M=\langle\dot{\beta}|\mu \cdot M / 2 n| \alpha\rangle$, $\hat{B}$ is a unit vector along $\vec{B}$ and $D_{+}=\left(\gamma / 2-i \omega_{\beta_{0}}\right)$. The constants $V_{O}$ and $U_{0}$ have numerical values $\mathrm{V}_{\mathrm{o}} \sqrt{3}$ ea $/ \hbar=13.93 \times 10^{6} \mathrm{sec}-1 / \mathrm{V}\left(\mathrm{cm}^{-1}\right)$ and $\mathrm{U}_{\mathrm{o}}=\mu_{\mathrm{o}} /=8.79 \mathrm{x}$ $10^{6} \mathrm{sec}^{-1} /$ gauss. We concentrate on the $\alpha_{0}-\beta_{0}$ amplitudes in the second cavity since the sum of all $\alpha_{0} \rightarrow \beta_{0}$ amplitudes in the first cavity can be combined into an overall amplitude $A_{I}$ which then interferes with the various amplitudes in the second cavity (this is strictly correct only if the various amplitudes in the final cavity are constant over the cross section of the beam). Let us decompose all the $E$ and $B$ fields into applied, fringing, motional and stray components. For example in an obvious notation $\vec{E}=\vec{E}_{a}+\vec{E}_{f}+\vec{E}_{m}+\vec{E}_{s}$ where $\vec{E}_{m}=$ $\vec{v} / \mathrm{c} \times \overrightarrow{\mathrm{B}}$. The matrix elements involving the fields are listed in Table 2.3.B-1.

In our geometry the applied external fields, $\vec{E}_{a}, \vec{E}_{a} I, \vec{R}_{a} I$ and $\vec{R}_{a} I I$ are ideally parallel to $\hat{B}$ and the symmetry axis of the atomic beam also lies along $\hat{B}$. This is possible in an apparatus which is perfectly aligned but which contains fringing fields and a finite emittance of the atomic beam. If the alignment were ideal in this sense all the spurious amplitudes 1isted in Table 2.3-1 either vanish by cylindrical symmetry or are cancelled by the reversals of $\vec{B}$ and $\phi$. However, the alignment cannot be perfect. In the following 
Table 2.3-1: $\quad \alpha_{0} \beta_{0}$ amplitides invoived in tne 570 gauss parity mixing measurement

\begin{tabular}{|c|c|c|c|c|c|c|c|c|}
\hline \multirow[b]{2}{*}{ Amplitude } & \multirow[b]{2}{*}{ Formb } & \multirow[b]{2}{*}{$\begin{array}{c}\text { Numerical Value } \\
\left.\text { iUnits of } \mathrm{R}_{\mathrm{a}} \mathrm{T}\right)\end{array}$} & \multicolumn{5}{|c|}{ Subtractions } & \multirow[b]{2}{*}{$\begin{array}{l}\text { Value } A \text { After } E \text {, } \\
\delta \text { and } \phi \text { subtraction }\end{array}$} \\
\hline & & & $\begin{array}{c}\text { Phase } \\
\text { Setting } \\
\end{array}$ & $\begin{array}{l}\text { Cyl. } \\
\text { Symn. }\end{array}$ & $\begin{array}{c}B \\
+ \\
-B \\
\end{array}$ & $\begin{array}{c}\delta \\
+ \\
-\delta \\
\end{array}$ & $\begin{array}{c}\alpha \rightarrow \beta \\
\downarrow \\
\beta \rightarrow \alpha\end{array}$ & \\
\hline${ }^{A} \mathrm{PV}$ & $i\left(C_{2}\right) \frac{V_{P V} V_{D}\left(\vec{R}_{a} \cdot \vec{B}\right)}{J_{0}} T$ & $=\times 10^{-9} C_{2} e^{i 60^{\circ}}$ & 1.00 & - & & & & $3 \times 10^{-9} \mathrm{c}_{2}$ \\
\hline$A_{p}$ & $\begin{array}{l}0.055 \frac{\mathrm{v}_{0}^{2}\left(\overrightarrow{\mathrm{E}}_{\mathrm{a}} \cdot \hat{\mathrm{B}}\right)\left(\overrightarrow{\mathrm{E}}_{\mathrm{a}} \cdot \hat{\mathrm{B}}\right)}{\mathrm{D}_{\mathrm{a}}} \mathrm{T} \\
0.055 \frac{\mathrm{v}_{\mathrm{o}}^{2}\left(\overrightarrow{\mathrm{R}}_{\mathrm{a}} \cdot \hat{\mathrm{B}}\right)\left(\overrightarrow{\mathrm{E}}_{\mathrm{s}} \cdot \hat{\mathrm{B}}\right)}{\mathrm{D}_{0}} \mathrm{~T}\end{array}$ & $\begin{array}{l}2.9 \times 10^{-2}\left(\vec{E}_{a} \cdot \hat{\mathrm{B}}\right) \mathrm{e}^{-\mathrm{i} 30^{\circ}} \\
2.9 \times 10^{-5} \mathrm{e}^{-\mathrm{i} 30^{\circ}}\end{array}$ & $10^{-4}$ & & $\mathrm{x}$ & & $\mathrm{x}$ & $\begin{array}{l}.0 \\
2.9 \times 10^{-13}\end{array}$ \\
\hline $\mathrm{A}_{\mathrm{J}_{1}}$ & 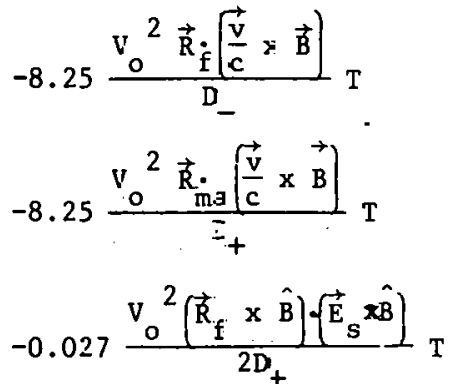 & $\begin{array}{l}-7.9 \times 10^{-5} e^{-i 50^{\circ}} \\
-4.7 \times 10^{-7} e^{-i 50^{\circ}} \\
-1.8 \times 10^{-7} e^{-i 50^{\circ}}\end{array}$ & -0.33 & $x$ & . & $x$ & $\mathrm{x}$ & $\begin{array}{l}7.8 \times 10^{-11} \\
1.5 \times 10^{-9}\end{array}$ \\
\hline
\end{tabular}


Table 2.3-1 (Continued)

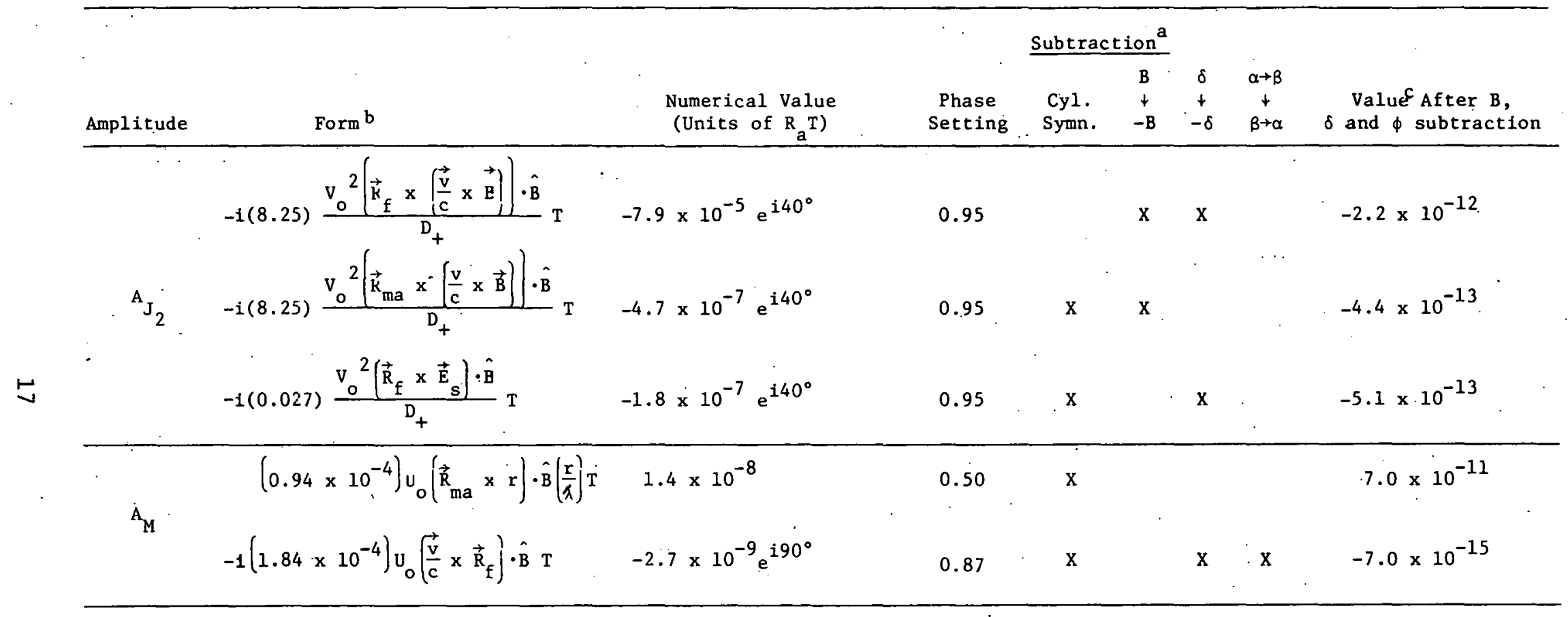

${ }^{a}{ }_{A n} X$ indicates that the corresponcing quantity is reduced by the subtraction.

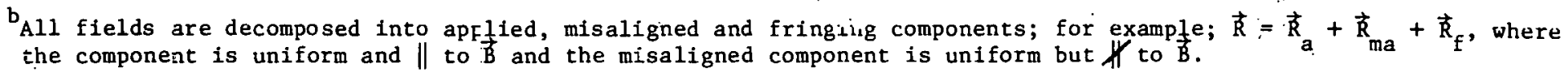

Reduction factors of $10^{-2}, 10^{-4}$ and $3 \times 10^{-4}$ are assumed for the subtractions due to cylindrical symmetry, $B$ and $\delta$. 
discussion we make a first order approximation--i.e., we assume the variou fields are constant but not colinear. Let the angle between $\vec{R}^{I I}$ and $\hat{B}$ be $\theta_{R}$ and between $\mathrm{E} I$ and $\hat{B}$ be $\theta_{\mathrm{E}}$. It is easy to see that both $A_{J_{1}}$ and $A_{J_{2}}$ are proportional to $\theta_{\mathrm{E}} \theta_{\mathrm{R}^{-}}-\mathrm{i} . \mathrm{e}$. , second order in small quantities. In addition $A_{M} \theta_{R}$ since $M_{\perp} R$ in the $T M_{010}$ mode and $R \|$ to $B$. Furthermore the induced motional $\hat{M}$ seen in the rest frame of the atoms $\hat{M}_{m}=-\vec{v} / c x$ is also small since $\vec{v}$ lies along $\vec{R}$. As we shall see later the $M 1$ contributions are small for other reasons as well. This favorable situation should be contrasted with those occurring in the schemes discussed in Refs. 9, 10, and 11 all of which involve applied transverse fields and hence contain systematic errors which are first order in misalignment angles.

The most important systematic error involves the motional field. At $500 \mathrm{eV}$ a H(2s) atom liavelling at an angle of 1 milliradian to a 570 gauss B fleld experiences a field $E_{m} \sim 0.18 \mathrm{~V} / \mathrm{cm}$, while for $C_{2}=0.05$ (the value predicted by the WS theory with $\sin ^{2} \theta_{w}=0.23$ ) the weak matrix element is equivalent to a field of $5 \times 10^{-9} \mathrm{~V} / \mathrm{cm}$. The motional field contribution to the amplitudes $\mathrm{AJ}_{1}$ and $\mathrm{A}_{\mathrm{J}_{2}}$ are

$$
\mathrm{A}_{\mathrm{J}_{1}}^{\mathrm{m}}=-8.25 \cdot \mathrm{V}_{\mathrm{O}}^{2} \mathrm{R} \cdot(\mathrm{v} / \mathrm{c}) \times \overrightarrow{\mathrm{B}} \mathrm{t}_{2} / \mathrm{D}_{+}
$$

and

$$
A_{J_{2}}^{m}=-i 8.25 v_{o}^{2}((\vec{v} / c \cdot \hat{B})(\vec{R} \cdot \vec{B})-|B|(\vec{R} \cdot \vec{v} / c)) t_{2} / D_{+}
$$

Under reversal of the direction of $\vec{B}$

$$
\mathrm{A}_{\mathrm{J}_{1}}^{\mathrm{m}} \rightarrow-\mathrm{A}_{1} \quad \text { while } \quad \mathrm{Ag}_{2} \rightarrow \mathrm{A}_{2}^{\mathrm{m}} \text {. }_{1}
$$

Therefore the $A_{J_{2}}^{m}$ term is greatly reduced by the subtraction associated with the reversal of $\vec{B}$, while $A_{J_{1}}^{m}$ is not affected.

Since $A_{J_{1}}^{m}$ is responsible for the most serious systematic errors in our scheme it is worth considering in some detail. If $\vec{R}$ were always parallel to $\hat{B}$, $\mathrm{AJ}_{1}$ would vanish identically. However because of fringing fields at the ends of ${ }^{1}$ the cavity an atom travelling parallel to $\hat{B}$ a distance $r$ from the axis of the cavity experiences an unavoidable $R_{\perp}$ from each end given by $\int R_{\perp} d t / \int R_{z} d t=r / 2 L$ where $L$ is the length of the cavity. If the atomic beam were not quenched by $R F$ quenching and if each atom in the beam had a constant value $r$, then on resonance, the effects of the two ends would cancei on an atom by atom basis. But since neither of these conditions is satisfied the cancellation is ineffective. On the other hand, if $\vec{k}, \vec{B}$, and the atomic beam have cylindrical symmetry around a common axis $A_{J_{1}}^{m}$ does vanish on an atom by atom basis.

To reduce the spurious amplitudes such as $A_{J}^{m}$ to a sufficiently small level one must align $\vec{R}$ and the atomic beam with $\hat{B}$ and minimize the effects of fringing fields. The average value $\widehat{R}_{\overparen{L}}$ of $\mathrm{R}_{\mathrm{E}}$ and $\mathrm{V}_{\mathrm{m}}$ can be efficiently minimized as follows. Set $\vec{B}$ to the $\alpha_{0} \rightarrow e_{0} \rightarrow \beta_{-}$resonance at 603.8 gauss. The amplitude for this resonance is $A_{0 x} \beta_{-}=-0.5 v_{0} 2\left|R_{z}\right|\left|E_{1}\right| t_{2} D_{-}$where $D_{-}=(\gamma / 2-$ 
$\left.i \omega_{\beta_{-}}\right)$. Vary the direction of the atomic beam so as to minimize $A_{\alpha_{0}} \beta_{-}$. This provides a sensitive way to align $\vec{B}$ with the symmetry axis of the atomic beam. Then align the RF cavity with $\vec{B}$ by returning to the $\alpha_{0} \rightarrow \beta_{0}$ resonance. Deliberately apply a strong transverse electric field $\frac{Q}{E}$. Then vary the alignment of the cavity with respect to $\hat{B}$ to minimize the amplitude $\mathrm{A}_{\mathrm{J}_{2}}=$ $-0.0275 \mathrm{~V}_{0}{ }^{2}|\mathrm{Rl}| \quad\left|\mathrm{E}_{\perp}\right| \mathrm{T} / \mathrm{D}_{+}$. This procedure greatly magnifies the effects of any misalignment and allows one to measure and correct the misalignment.

The effects of the fringing fields at the cavity ends can be cancelled by running on the sides of the $\alpha_{0} \rightarrow \beta_{0}$ resonance $(\delta=\emptyset)$. off resonance the phase of the $\alpha_{0} \rightarrow \beta_{0}$ amplitude depends upon $z$, the position inside cavity where the transition occurs. Therefore the phases of amplitudes generated in the beginning and end of cavity (e.g., from fringing fields) are different from the phase of an amplitude which is generated throughout the cavity (e.g., APNC). One can always choose $\delta$ in such a way that when $\delta \rightarrow-\delta$ the amplitude driven by fringing fields does not change its phase relative to some constant interfering amplitude (e.g., $A_{P C}$ ) while the amplitude driven by the applied field will change sign. For simplicity consider an ideal situation where the fringing fields have negligible width, the applied fields are small and the phase is chosen to be zero if the transition occurs in the beginning of cavity. Then the

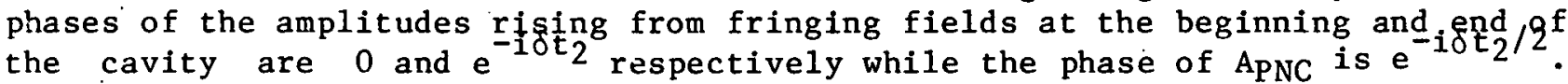
So if $\delta t_{2}$ is chosen to be $t \pi$ then as $\delta \rightarrow-\delta$, the amplitudes driven by fringing fields do not change their phase but $A_{P N C}$ changes its sign. This can be used to cancel the effects of the fringing fields. The cancellation is limited by an unavoidable variation in times $t_{2}$ due to a spread in the velocities of the atomic beam. A duoplasmatron produces a velocity spread $\Delta v / v \sim 10^{-2}$. This ultimately limits the cancellation to a factor of $(\Lambda t / t)^{2}=(\Lambda v / v)^{2} \sim 10^{-4}$ even if the frequency shift $\delta$ could be chosen exactly.

There is, in principle, a fifth subtraction which can be very useful in further reducing systematic errors. This subtraction is based on the imaginary nature of the weak matrix element $V_{P N C}$. Time reversal invariance requires. that a11 amplitudes which contain an odd number of T-odd factors ( $\vec{B}^{\prime} s$ and $\vec{v}^{\prime} s$ ) must also contain a factor of $i$, for example APNC contains one factor of $B$ and has a factor of $i$, while $A_{p}$ contains two factors of $B$ and no factor of $i$. Amplitude with this factor of $i$ will change sign when initial and final states are reversed $\left(\alpha_{0} \rightarrow \beta_{0}\right.$ is changed to $\left.\beta_{0} \rightarrow \alpha_{0}\right)$, while those without this factor of $i$ do not. Now compare the effects of the transformations $B \rightarrow-B$ and $\alpha \beta \rightarrow \beta \alpha$. All amplitude which do not contain an odd power of beam velocity behave similarly under the two transformations. However those amplitudes which do contain a factor of the beam velocity will transform oppositely under $\vec{B} \rightarrow-\vec{B}$ and $\alpha \beta \rightarrow \beta \alpha$. Hence all motional field amplitudes are either cancelled by the subtraction $\vec{B} \rightarrow-\vec{B}$ or $\alpha \beta \rightarrow \beta \alpha$. This behavior is shown more clearly in Table $2.3 . B-1$. The subtraction $\alpha \beta \rightarrow \beta \alpha$ can be implemented by alternating between $R F$ and static $\vec{E}$ field quenching before the main $\mathrm{RF}$ cavity and the $\mathrm{L}_{\alpha}$ detector. 
It is obvious that further reduction of the systematic errors would b achieved if one could reduce the velocity $\mathrm{v}$ of the atomic beam. Intense thermal beams of $\mathrm{H}(2 \mathrm{~s})$ are being developed.13 A thermal beam would reduce al1 motional field effects by a factor of $\sim 100$. In principle it would also increase the PNC effect by a factor of $\sqrt{T} \propto 1 / \sqrt{v} \sim 10$. However to obtain full benefit of this factor of 10 the magnetic field must be uniform and stable to better than 4 parts in $10^{5}$.

Numerical estimates of the various amplitudes discussed above are presented in Table 2.3.B-1 for the case of a $500 \mathrm{eV}$ atomic beam without the $\alpha \beta \rightarrow \beta \alpha$ subtraction. It seems likely that a thermal atomic beam of the $\beta \rightarrow \beta$ subtraction will be necessary to reach a sensitivity in $C_{2}$ as small as that predicted by the wS theory.

We gratefully acknowledge informative conversations about this experiment with a large number of colleagues. We are particularly indebted to Prof. E.A. Hinds for introducing us to attractive features of two cavity experiments and tor helping to clarity some issues concerning systematic errors in measurements of this type.

D. Considerations Involving a Possible Hydrogen Atom Parity Mixing Experiment in Zero Magnetic Field

E.G. Adelberger; E.N. Fortson, and M.Z. Iqbal

In hydrogen atom the $S$ states have small admixture of $P$ states because of weak interactions. In a magnetic field around $570 \mathrm{G}$ the Zeeman splitting makes the $2 \mathrm{~S}\left(\mathrm{~m}_{\mathrm{J}}=-1 / 2\right)$ states degenerate with the decaying $2 \mathrm{P}\left(\mathrm{m}_{\mathrm{J}}=1 / 2\right)$ states. Because of vanishing energy denominator the admixture is maximum at this point. This was one of the reasons for doing the experiment in a magnetic field. But for a given length of time the statistical signal to noise ratio depends only on the parity nonconserving amplitude and is independent of parity conserving amplitude.14 It turns out that for a given magnetic field one can always optimize the parity nonconserving amplitude by adjusting the RF electric field.

If a transition is driven to state

$$
|\beta\rangle+\left|\beta_{0}\right\rangle+\frac{\left.\kappa_{0}\left|H_{P N C} / \beta_{1}\right| \beta_{0}\right\rangle}{{ }_{B_{0} e_{0}}+\frac{i \gamma}{2}}\left|e_{0}\right\rangle
$$


from state $\left|\alpha_{0}\right\rangle$ near $570 \mathrm{G}$ applying an $R F$ electric field $\left(R e^{-i \omega t}+R^{\star} e^{i \omega t}\right.$ ) then on resonance $\left(\omega=\omega_{\alpha_{0}}{ }^{-\omega_{\beta_{0}}}\right)$ the $|\beta\rangle$ amplitude is given by

$$
\beta_{0}\left(\alpha_{0} \stackrel{\mathrm{H}_{\mathrm{E}}}{\rightarrow} \mathrm{e}_{\mathrm{o}} \stackrel{\mathrm{H}_{\mathrm{PNC}}}{\rightarrow} \beta_{\mathrm{o}}\right)=-\frac{1 \overline{\mathrm{C}}_{2} \overline{\mathrm{R}}_{z}}{\frac{\gamma}{2}-i \omega_{\beta_{0} e_{0}}}\left(\frac{1-\mathrm{e}^{-\Gamma \mathrm{t}}}{\Gamma}\right)
$$

where

$$
<\beta_{0}\left|\frac{H_{\mathrm{PNC}}}{\hbar}\right| e_{\mathrm{o}}>=i \overline{\mathrm{C}}_{2}, \quad\left\langle\mathrm{e}_{\mathrm{o}}\left|\mathrm{H}_{\mathrm{E}}\right| \beta_{\mathrm{o}}>=\mathrm{e}_{\mathrm{o}}\left|\mathrm{e} \frac{\overrightarrow{\mathrm{R}} \cdot \overrightarrow{\underline{r}}}{h}\right| \beta_{\mathrm{o}}>=\overline{\mathrm{R}}_{z},\right.
$$

$\gamma / 2$ is the $2 P$ state decay rate and

$$
\Gamma=\frac{\gamma}{2} \frac{\overline{\mathrm{R}}_{z}^{2}}{\left(\frac{Y}{2}\right)^{2}+\left(\omega_{\alpha_{0} \mathrm{e}_{0}}-\omega_{\alpha_{0} \beta_{0}}\right)^{2}}
$$

The notation for the states involved is established in Figure 2.3.A-1. To genefalize for arbitrary magnetic field we also have to include the $\beta_{0}$ amplitude $\left(\alpha_{0} \underset{\mathrm{E}}{\rightarrow} e_{0} \underset{{ }_{P N C}}{\rightarrow} \beta_{0}\right)$ and similar amplitudes generated through intermediate $f$ states. In calculating the optimum RE. strength we will neglect these other amplitudes since they do not have a large effect. The condition for optimum $\beta_{0}$ with respect to $R$ (in a magnetic field for a given time $t$ ) is found from $\partial \beta_{0} / \partial R$ $=0$ which gives rise to the condition

$$
e^{-\Gamma t}(1+2 \Gamma t)=1 .
$$

One can see that $\Gamma t \simeq 1.25$ and $R^{2} \simeq\left[(\gamma / 2)^{2}+\left(\omega \beta_{0} e_{o}-\omega \beta_{o} e_{o}\right)^{2}\right](g / 2) t$. This shows that optimum $\beta_{0}$ is sane for any magnetic field $q_{i}^{e} e^{e}$, any $\omega_{\beta_{0}} e_{o}$ ) and one can always find $a \cdot R$ to optimize $\beta_{o}$ at any magnetic field. Near $570 G$ $\left(\omega_{\beta_{0}} e_{0} \simeq 0\right) \quad R=20 \times 10^{6} \mathrm{sec}^{-1}$ only but at zero field $\left(\omega_{\beta_{0}} e_{o} \gg \gamma / 2\right), R=560 \times$ $10^{\circ} \mathrm{sec}^{-1}$ for $t=1 \mu s$.

In zero magnetic field $F$ and $m_{F}$ are good quantum numbers $(\vec{F}=\vec{I}+\vec{J})$ and one can calculate the $\mathrm{H}_{\mathrm{PNC}}$ matrix elements in the basis $\left|\mathrm{F}, \mathrm{m}_{\mathrm{F}}\right\rangle$ 


$$
\begin{aligned}
& <F^{\prime}, m_{F}^{\prime}\left|\left(\psi_{2 p^{\prime}}, \frac{{ }_{P N C}}{\hbar} \psi_{2 s}\right)\right| F, m_{F}=i \delta_{F F}, \delta_{m_{F} m_{F}^{\prime}} v_{P N C}\left(C_{1}-C_{2}\right) . \quad F \neq 0
\end{aligned}
$$

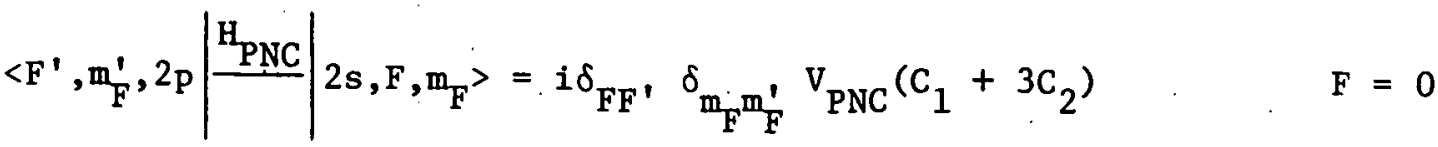

The useful relation between the stark $\left(H_{E}\right)$ and magnetic $\left(H_{B}=-\vec{\mu} \cdot \vec{B}\right)$ matrix clement is

$$
\left\langle\mathrm{F}^{\prime} \mathrm{m}_{\mathrm{F}}^{\prime}, 2 \mathrm{p}\left|\mathrm{H}_{E(B)}\right| 2 s(2 \mathrm{p}), \mathrm{F}, \mathrm{m}_{\mathrm{F}}\right\rangle=\left|\mathrm{F}^{\prime} \mathrm{m}_{\mathrm{F}}^{\prime}, 2 \mathrm{~s}\right| \mathrm{H}_{\mathrm{E}(\mathrm{B})}\left|2 \mathrm{p}(2 \mathrm{~s}), \mathrm{F}, \mathrm{m}_{\mathrm{F}}\right\rangle
$$

The exact matrix elements are shown in Fig. 2.3. D-1.
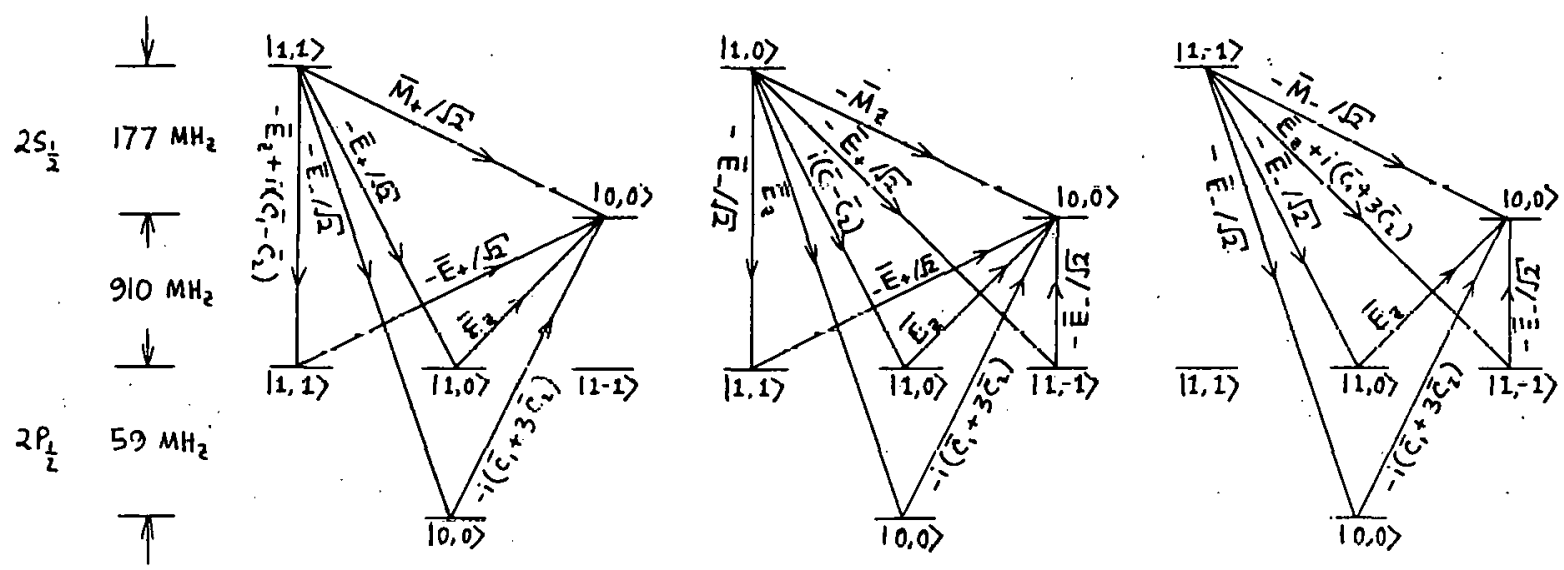

Fig. 2.3.D-1: Transition to $\beta_{0}$ state from $\alpha_{+}, \alpha_{0}$, and $\beta_{-}$states (through the intermediate $e_{+}, e_{o}, f_{-}$, and $f_{o}$ states). due to RF electric and magnetic fields in static magnetic field. 
In zero magnetic field, the parity nonconserving amplitude vanishes unless $\Delta F=1$. For example the $2 \mathrm{~S}\left|1, \mathrm{~m}_{\mathrm{F}}\right\rangle \overrightarrow{\mathrm{f}} \mathrm{S}|0,0\rangle$ occurs while all other amplitudes, such as $2 \mathrm{~S}\left|1, \mathrm{~m}_{\mathrm{F}}\right\rangle \supsetneqq 2 \mathrm{~S}\left|1, \mathrm{~m}_{\mathrm{F}}\right\rangle$ vanish. For example, on resonance

$$
\begin{aligned}
& A_{P N C} 2 s\left|1, m_{F}\right\rangle \rightleftarrows 2 s\left|1, m_{F}^{\prime}\right\rangle=\frac{\left\langle 1, m_{F}^{\prime}, 2 s\left|\frac{H_{P N C}}{h}\right| 2 p, 1, m_{F}^{\prime} \times 1, m_{F}^{\prime}, 2 \mathrm{p}\left|\frac{H_{E}}{h}\right| 2 s, 1, m_{F}\right\rangle}{\frac{Y}{2}-i \omega_{s 1, p 1}} t \\
& +\frac{\left\langle 1, \mathrm{~m}_{\mathrm{F}}^{\prime}, 2 \mathrm{~s}\left|\frac{\mathrm{H}}{\mathrm{f}}\right| 2 \mathrm{p}, 1, \mathrm{~m}_{\mathrm{F}} \times \mathrm{m}_{\mathrm{F}}, 2 \mathrm{p}\left|\frac{\mathrm{H}_{\mathrm{PNC}}}{\hbar}\right| 2 \mathrm{~s}, 1, \mathrm{~m}_{\mathrm{F}}\right\rangle}{\frac{\gamma}{2}-i \omega_{\mathrm{s}_{1} \mathrm{p}_{1}}} t
\end{aligned}
$$

The HPNC matrix elements are completely imaginary and the first term has negative sign relative to the second. The $\mathrm{H}_{\mathrm{E}}$ matrix elements are the same in both terms so that the amplitude vanishes.

Using the matrix elements given in Fig. 2.3.D-1 one can easily find the $2 S|1, m\rangle \rightarrow 2 S|0,0\rangle$ amplitudes, $\beta_{0}{ }^{m}$ driven by resonating $\left(\omega=\omega_{s 1}-\omega_{s o}\right) R F$ magnetic field and resonating RF electric field. .

$$
\begin{aligned}
& \beta_{0}^{1}=\left(\frac{i(\vec{K} \times \vec{E}) z}{\Delta o}-i \bar{R}_{z}\left(\frac{\bar{C}_{1}}{\Delta_{1}}+\frac{\bar{C}_{2}}{\Delta_{2}}\right)-\bar{M}_{z}\right) t \\
& \beta_{0}^{1}=\left(\frac{-i(\vec{R} \times \vec{E})}{\sqrt{2} \Delta o}+\frac{i \bar{R}_{t}}{\sqrt{2}}\left(\frac{\bar{C}_{1}}{\Delta_{1}}+\frac{\bar{C}_{2}}{\Delta_{2}}\right)-\frac{1}{\sqrt{2}} \bar{M}_{t}\right) t \\
& \beta_{0}^{-1}=\left(\frac{i(\vec{R} \times \vec{E})}{\sqrt{2} \Delta o}-\frac{i \bar{R}}{\sqrt{2}}\left(\frac{\bar{C}_{1}}{\Delta_{1}}+\frac{\bar{C}_{2}}{\Delta_{2}}\right)-\frac{1}{\sqrt{2}} \bar{M}_{-}\right)_{t}^{t}
\end{aligned}
$$

where $\omega_{S 1}, \omega_{S 0}, \omega_{P 1}, \omega_{P 0}$ are the angular frequencies: corresponding to the energy of the states $2 S(F=1,0)$ and $2 P(F=1,0)$, respectively. 


$$
\begin{aligned}
& \frac{1}{\Delta_{0}}=\frac{1}{\frac{\gamma}{2}-i \omega_{s_{0} p_{1}}}-\frac{1}{\frac{\gamma}{2}-i \omega_{s_{1} p_{1}}} \\
& \frac{1}{\Delta_{1}}=\frac{1}{\frac{\gamma}{2}-i \omega_{s_{0} p_{0}}}-\frac{1}{\frac{\gamma}{2}-i \omega_{s_{1} p_{1}}} \\
& \frac{1}{\Delta_{2}}=\frac{1}{\frac{\gamma}{2}-i \omega_{s_{0} p_{0}}}-\frac{1}{\frac{\gamma}{2}-i \omega_{s_{1} p_{1}}}
\end{aligned}
$$

and $\vec{R}(\vec{E})=\sqrt{3}$ ea $/ \hbar \frac{\hbar}{R}(\vec{E}), \vec{M}=\mu_{o} / \hat{n} \vec{M}, \vec{R}(\vec{E})$ is $R F$ (static) electric field and $\vec{M}$ is $R F$ magnetic field.

For a system without any preferred direction where all $\psi_{2 S}|1, m\rangle$ states are equally populated the net transition to $\psi_{2 S}|00\rangle$ is given by

$$
\begin{aligned}
\left|\beta_{0}\right|^{2}= & \left|\beta_{0}{ }^{2}\right|^{2}+\left|\beta_{o}^{o}\right|^{2}+\left|\beta_{o}^{-1}\right|^{2}=t^{2}\left(\frac{\mid \overrightarrow{\mathrm{R}} \times \overrightarrow{\mathrm{E}}^{2}}{\left|\Delta_{\mathrm{o}}\right|^{2}}+\overline{\mathrm{R}}^{-2}\left(\frac{\overline{\mathrm{C}}_{1}}{\Delta_{1}}+\frac{\overline{\mathrm{C}}_{2}}{\Delta_{2}}\right)^{2}\right. \\
& \left.\overline{\mathrm{M}}^{2}+2 \mathrm{~T}_{\mathrm{m}}\left(\frac{\overrightarrow{\mathrm{M}}}{\mathrm{M}} \cdot \frac{\overrightarrow{\mathrm{R}} \times \overrightarrow{\mathrm{E}}}{\Delta_{o}}\right)+2 \mathrm{I}_{\mathrm{m}} \mid \mathrm{M} \times \mathrm{R}\left(\frac{\overline{\mathrm{C}}_{1}}{\Delta_{1}}+\frac{\overline{\mathrm{C}}_{2}}{\Delta_{2}}\right)\right)
\end{aligned}
$$

A little inspection of this expression shows that the zero field experiment can be divided in two classes: 1) without initial state selection (observable pseudoscalar $\vec{M} \cdot \vec{R}$ ); 2 ) with initial state selection (observable pseudoscalar $\vec{k} \cdot \hat{n}$ $\overrightarrow{\mathrm{R}} \cdot \hat{\mathrm{n}}$, unit vector $\hat{\mathrm{n}}$ determined by the state selected.)

1) One can design an experiment without choosing any particular state in various ways, but from the systematic point of view they are all equivalent. We consider the simplest case with coherent $R F$ magnetic and electric field, $\vec{M}$ and $\vec{R}$, in two separate regions, $T$ and II respuctivcly. $M$ and $R$ will generate parity nonconserving amplitude respectively. The relative phase $\phi$ between $\vec{M}$ and $\vec{R}$ could be adjusted for maximum interference between the amplitudes. The total production of $\left|\beta_{0}\right\rangle$ state is given by 


$$
\begin{aligned}
& \left|\beta_{0}\right|^{2}=\left(\left|\frac{\alpha \hat{\mathrm{x}} \cdot \overrightarrow{(\overrightarrow{\mathrm{R}} \times \overrightarrow{\bar{B}}) L}}{477 \lambda}\right|^{2}+2 \alpha \operatorname{Re} \frac{\hat{\hat{\mathrm{x}}} \cdot \overrightarrow{(\overrightarrow{\mathrm{R}} \times \overrightarrow{\mathrm{B}}) \mathrm{L}}}{4777 \lambda}(\overrightarrow{\overline{\mathrm{R}}} \cdot \overrightarrow{\overline{\mathrm{B}}})\left(\frac{\overrightarrow{\mathrm{C}_{1}}}{\Delta_{1}}+\frac{\overline{\mathrm{C}}_{2}}{\Delta_{2}}\right)\right. \\
& \left.+2 \alpha \operatorname{Re} \frac{\hat{\hat{x} \cdot\left(\frac{\vec{R} \times \bar{B}}{\vec{R}}\right) L}}{477 \lambda} \bar{E}_{s}\left(\frac{\frac{ \pm}{R} \times \bar{B}}{\Delta_{0}}\right)\right) t^{2}
\end{aligned}
$$

where $\vec{E}_{S}$ is a stray electric field, $|\vec{r}|$ is the bean radius and $\dot{\lambda}=c / \omega$. Smaller terms are neglected in the expression. The signal is the charge in the $\alpha \rightarrow \beta_{0}$ transition rate when $\phi \rightarrow \phi+h$. The dominant systematic error arises from $M 1$ transitions due to the RF magnetic field in region II induced by the time vartation of $\vec{R}$. This places a severe limit on the sensitivity of the experiment. This zero field scheme is not more favorable than the 575 gauss Mark II experiment discussed above.

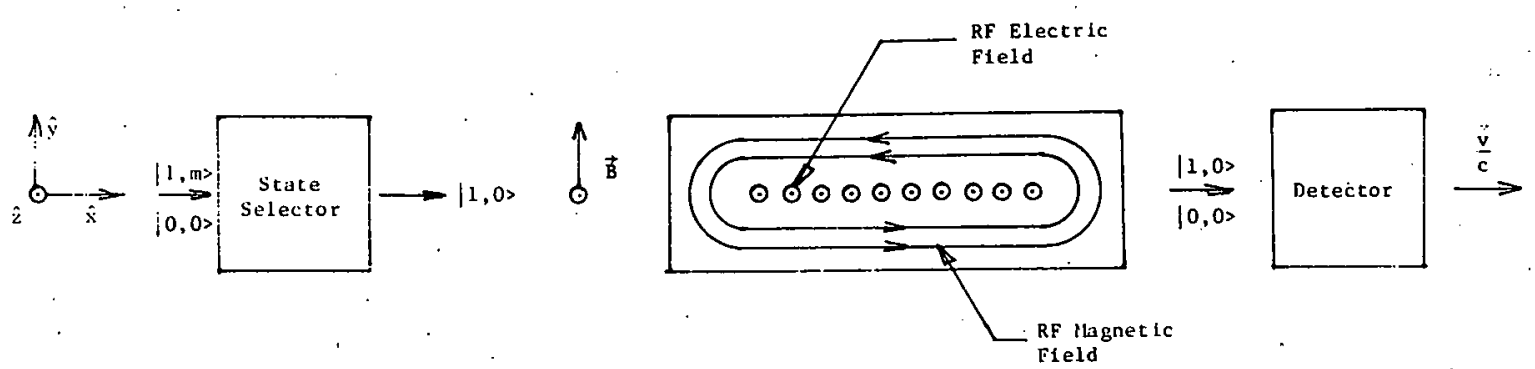

Fig. 2.3.D-2: The scheme of a zero magnetic field experiment using a definite initial state.

2) In this scheme one needs to choose a particular state (say $\left.\alpha_{0}\right\rangle$ ) with respect to a given direction (say $\hat{z}$, perpendicular to the beam direction $\hat{x}$ ). The direction can be tilted by a small amount in one direction (say $\hat{y}$ ) by applying a localized magnetic field $\vec{B}\left(\vec{B}=B_{0} \hat{e}_{z}+\Delta B \hat{e}_{y}\right)$ before the beam enters the transition region. The transition region contains an $R F$ electric field $\vec{R}$ along $\hat{z}$ direction and an $R F$ magnetic field $M=$ $i \vec{r} \times \vec{k} / 300 \lambda$ as shown in Fig. 2.3.D-2. $\vec{r}$ is the radius vector measured from the center of transition region along the beam. There are no static electric or magnetic fields in the function region. The small component of 
11 along $B$ will generate a nonzero parity conserving amplitude although the $\vec{M}$ reverses its direction along the paths, because of the decay of initial $\left|\alpha_{0}\right\rangle$ state. The parity nonconserving amplitude will be driven by $\vec{R}$. The total production of $\left|\beta_{0}\right\rangle$ is completely given by

$$
\begin{aligned}
& \left|\beta_{o}{ }^{0}\right|^{2}=t^{2}\left(\vec{M}^{2}-2 I_{m} \stackrel{\vec{M}}{M} \cdot \frac{\vec{R}}{R} e^{-i \phi} \cdot\left(\frac{\vec{C}_{1}}{\Delta_{1}}+\frac{\bar{C}_{2}}{\Delta_{2}}\right)+2 I_{m} \frac{\overrightarrow{(\vec{M} \times \bar{R})} \frac{\vec{F}}{F_{s}} e^{-i \phi}}{\Delta_{o}}\right. \\
& \left.+2 \operatorname{Re} \frac{\vec{r}\left(\frac{\vec{r}}{(i)} \vec{R}\right) e^{-1 \psi}}{477 \lambda}\right)
\end{aligned}
$$

where $L$ is the total length of transition region and $\alpha$ is related to the amount of the decay of beam. Reversing the sign of $\triangle B$ changes the sign of the parity conserving amplitude. This produces a detectable signal. By making $(\triangle B / B)$ small one can in principle reduce the only systematic error from stray electric fields. The accuracy in the state selection puts a limit on this experiment. Analysis of the systematic effects in this scheme is continuing.

\section{E. H-Atom Atomic Beam Studies}

E.G. Adelberger, T.E. Chupp, D. Holmgren, M.Z. Iqbal, and T.A. Trainor

Since our last report several systems in the hydrogen parity apparatus have been greatly improved, including:

1) Double detector and mirror system (30X increase in solid angle)

2) Cryopump system installed (two orders of magnitude decrease in system operating pressure; $\left(4 \times 10^{-7} \rightarrow 4 \times 10^{-9}\right.$ torr $)$

3) Installation of Helmholtz coils required to generate $\alpha_{n}-\beta_{n}$ and $\alpha_{-}-\beta_{-}$ reoonunces

4) Reduction of acoustical noise contributions to the detector signal by installing low noise coaxial cables.

With these and other improvements in the system (described in this Annual Report) we have been able to meet our first major goal of observing the $\alpha_{0}-\beta_{0}$ and $\alpha_{+}-\beta$ - resonances at a level of $-10^{-6}$ of the incident metastable flux. This is shown in the upper scale of Fig. 2.3.E-1, where the direct yield from the Lyman- $\alpha$. detector preamp is plotted vs. axial magnetic field strength. No lock-in amplifier was used for these scans. The vertical scale is different for the "allowed" $\alpha_{+}-\beta_{0}$ and $\alpha_{0}-\beta$ - resonances. 


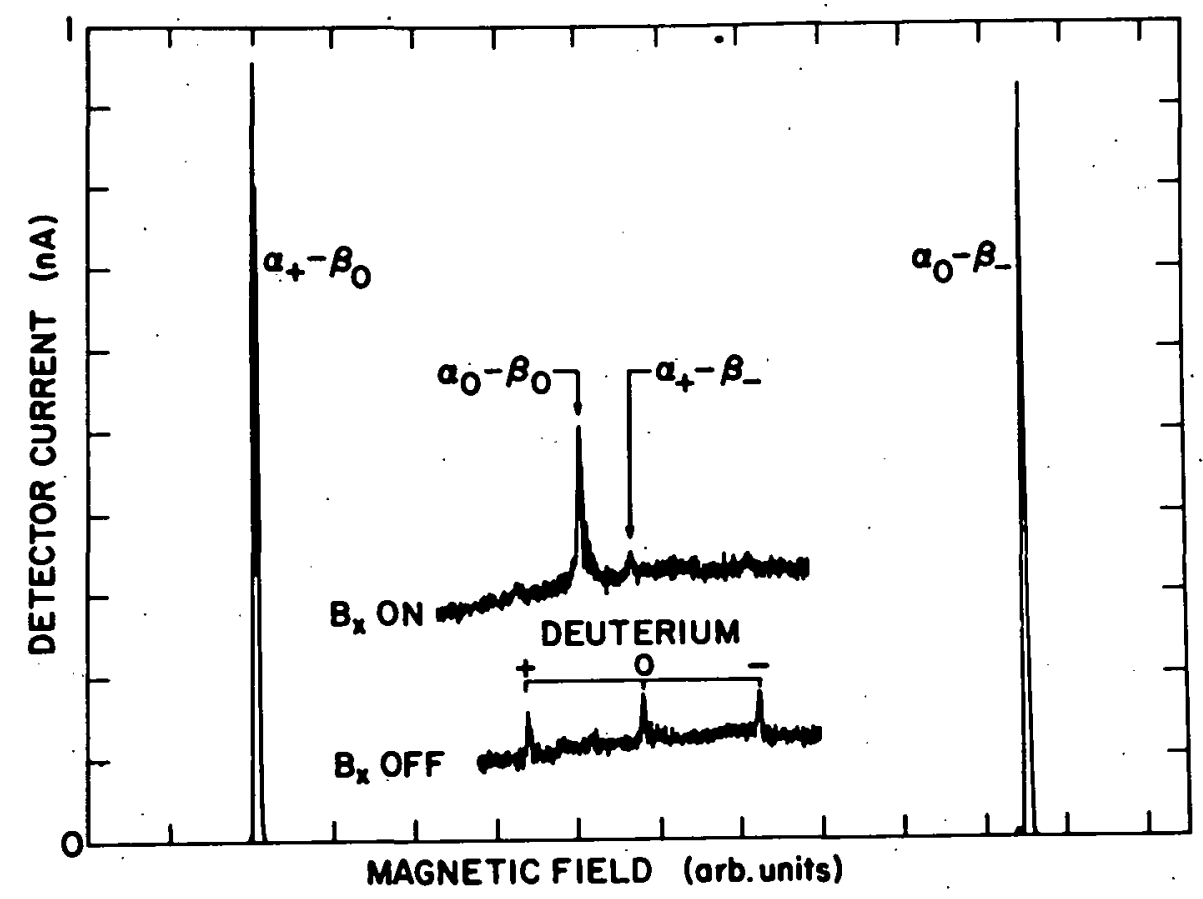

Fig. 2.3.E-1: B-state yield vs. axial magnetic field

Subsequent to this achievement we have studied the existing limitations of our apparatus, including the effects of "resonance distortions" in asymmetry measurements over the narrow resonances and the various sources of background light intensity seen by the $L-\alpha$ detectors. These and other sources of systematic error have to be understood and pushed down well below the anticipated level of the parity-violating asymetry. $\left(\sim 10^{-6}\right)$. The results of the past year's studies are reported below and have resulted in a major redesign of the main solenoid and RF cavities in the apparatus, described elsewhere in this Annual Report.

Backgrounds:

Presently about $50 \%$ of the detected Lyman- $\alpha$ light with all metastable atoms quenched far upstream is due to direct L- $\alpha$ light from the duoplasmatron arc. This is light that remains when the beam at the ion source is steered in such a way that it doesn't enter the solenoid. It is presumably multiply scattered light which has found its way to the detectors in spite of various apertures. The remaining signal $(40 \%)$ is associated with ground state atoms passing through the detector system. This signal is independent of upstream quenching fields and detector quench field and is most probably due to scattered atoms which hit a metal surface in view of the detector in spite of the beam collimation. Collimation of any beam with finite emittance is a delicate matter. 
Fig. 2.3.E-2: Resonance distortion from B-field variation.

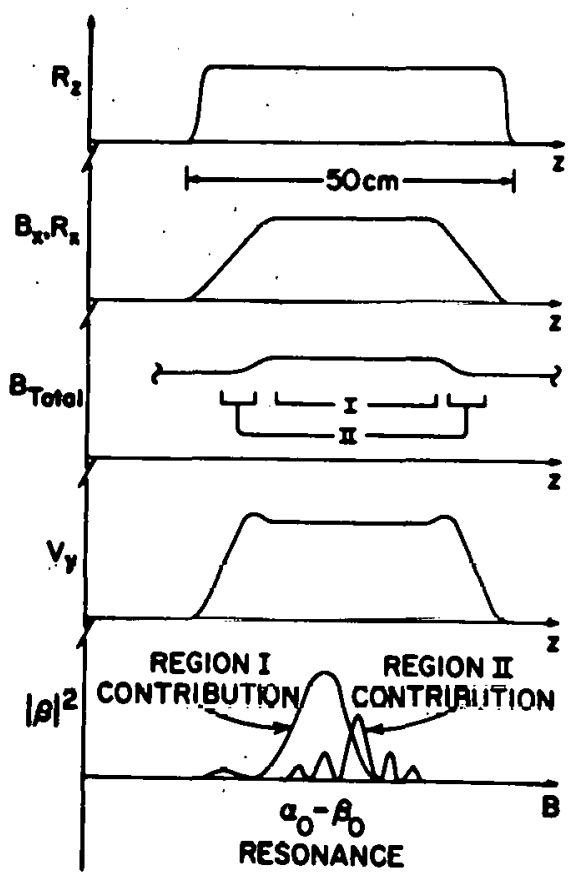

We also see clear evidence of direct $\alpha-\beta$ transitions induced by gas scattering. This background is considerably reduced by operating the $\alpha-\beta$ transition fields somewhat higher than optimum, indicating that the principle production area is upstream where (1) the $\alpha$-state intensity is much higher and (2) the pressure is about twice as high. We see no evidence of second-order processes (e.g., arclight-beam or beam-beam scattering) which would depend on

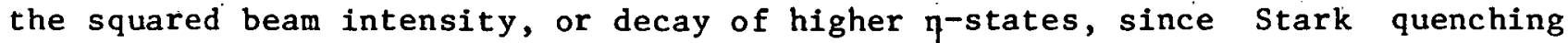
in a rising B-field upstream does not change the background intensity.

The present background intensity is about $700 \mathrm{fA}$ with unity gain detectors. By comparison the $\alpha_{0}-\beta_{0}$ and $\alpha_{+}-\beta_{-}$peaks (with $V_{y}$ ) are about 100 fA maximum.

Resonance bistortion:

This is a significant problem which we have recently encountered. It involves the dependence of a resonance shape on the variation of the electromagnetic field intensities along the path of the atom. 
As an example consider the $\alpha_{0}-\beta_{0}$ resonance in Fig. 2.3.E-1. This resonance is asymmetric--it has a series of fringes on one side. The explanation for this resonance distortion can be seen in Fig. 2.3.E-2. The axial $R F$ field $R_{z}$ rises and falls quickly and is uniform throughout the transition region. $B_{x}$ (and therefore $R_{x}{ }^{-}$) rises and falls slowly and is uniform over a smaller region. The total magnetic field B total now includes a $\sim 0.75 \mathrm{G}$ "plateau" due to the addition of $B_{x}$. The total electric field $V_{y}$ includes an applied field shaped very much like $B_{x}$ plus a motional field contribution at the ends due to a slight mismatch of $B_{x}$ and $v_{y}$ strengths there.

As the solenold current is increased region $I$ (defined in Fig. 2.3.E-2) is the first to pass through the resonance. In this region the fields are all uniform and continuous. A resonance with a dominant central peak and width corresponding to the length of region I results.

As the solenoid field is further increased region II passes through the resonance. However, this has two separate parts with highiy structured field intensities. The result, as in a separated-cavity device, is a fragmented resonance with fringe widths corresponding to the separation between the two parts of region II. These two contributions are superposed in the observed $\alpha_{0}-\beta_{0}$ resonance of Fig. 2.3.E-1.

This effect could be removed by trimming $B_{z}$ so that $B_{\text {total }}$ is uniform over the entire cavity when $B_{x}$ is on and by improving the $B_{x} V_{y}$ intensity match along the beam axis. It should be noted that with $B_{x}$ off resonance shapes are "reasonable" $\left(\alpha_{+}-\beta_{0}, \alpha_{0}-\beta_{-}\right)$. The effect is not present in the Mark II scheme since all fields are parallel to $B$.

The second effect which we have encountered is potentially much more serious. In brief, notional fields due to imperfections in the main solenoid $\mathrm{B}$-field induce changes in the resonance centroid, width, skewness and higher moments which are correlated with field reversals. The result is an observed asymmetry which is highly structured across the resonance.

In F'ig. 2.3.E-3 is shown a simplified example. Suppose the solenoid B-field has a transverse component which spirals about the axis along the solenoid length. The component in one plane might be as shown at the top of the figure. To the resulting motional E-field is added or subtracted a transverse static field $V$ in the same plane. The resulting total field has quite different shapes depending on the polarity $(t)$. In case $(+)$ there are two separate regions, resulting in a fragmented resonance. In case $(-)$ there is a single, 
Fig: 2.3.E-3: Resonance distortion:

Motional field variation.

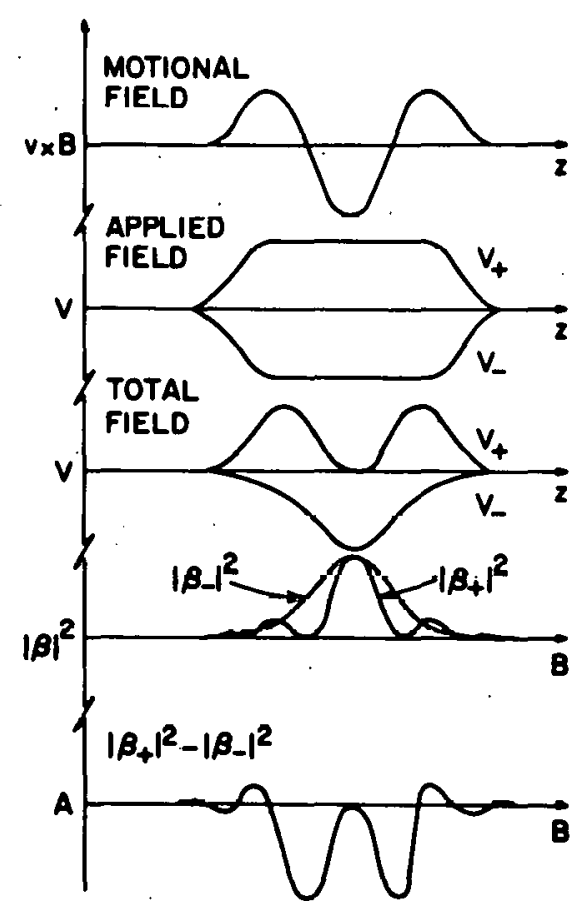

shorter region resulting in a single, broader resonance shape. If a yield asymmetry $\beta_{+}{ }^{2}-\beta_{-} 2$ is now calculated the result is as shown at the bottom of Fig. 2.3.E-3. In this case the magnitudes of $V_{+}$and $V_{-}$have been separately adjusted to give zero asymmetry "on resonance." However, any B-field drift will result in a non-zero asymmetry measurement. Such an asymmetry can easily be of order $\quad \beta 2$.

In general, any field reversal which involves a change in that fleld's intensity variation along the beam path will produce a resonance distortion and resulting complicated false asymmetry. In order to eliminate such an asymetry a field must be reversed without changing its magnitude at any point along the beam path. This puts a much more stringent requirement on various field homogeneities than we had anticipated. The problem is reduced if the resonance is broadened, by shortening the transition region and/or by power broadening.

In general we have found that much can be learned about systematics by going to one of the large resonance $\left(\alpha_{0}-\beta_{-}\right.$has the simplest interpretation) and seeing what happens when the static electric field is reversed. Ideally a zero asymmetry should result, independent of $B$.

These studies of resonance distortion effects have prompted us to redesign the main solenoid to reduce greatly the variation in magnitude and direction of transverse magnetic fields along the beam path (a uniform transverse field is a much less significant problem and can be cancelled to a great extent), and to redesign the interaction region so that (1) Stark and parity-violating amplitudes are built up in two separate $R F$ cavities (discussed elsewhere in this report), and (2) all applied EM fields are axial in the two cavities so that effects discussed here and related to unwanted transverse fields enter the problem in second order. 
F. Measurement of the Efficiency of a Lyman-x Detection System for the Hydrogen Parity Experiment

K.J. Davis, D. Holmgren, and T.A. Trainor

In two previous annual reports (1978, p. 20 and 1979, p. 36) we discussed the design of a pair of spherical mirrors used to enhance the detection of Lyman- $\alpha$ radiation produced by $\beta$-state quenching in the hydrogen parity experiment. The metastable hydrogen beam is quenched between two detectors, and the mirrors act to focus the light onto the detectors. Here we report on the performance of the detection system.

In order to determine the effectiveness of the mirrors, we removed one of them so that only one of the detectors received reflected, as well as direct, Lyman- $\alpha$ radiation. Assuming that the detectors have equal efficiency, any deviation from unity in the ratio of detector currents can be attributed to the presence of the mirrors and does not depend on the beam current. This measurement is insensitive to light that would enter the detector after multiple reflections within the mirror pair, but this is likely to be a small effect.

The ratio of currents of the detector plus mirror combination to the other detector was found to be $\sim 15$. Since the solid angle subtended by a single detector is $0.020 \times 2 \pi \mathrm{sr}$ this implies an effective solid angle of $0.31 \times 2 \pi$ sr for the mirror-detector combination (after correction for a $97 \%$ transparent screen in front of the lone detector). If we include a detector quantum efficiency of $42 \%$ (manufacturer's estimate) we obtain an overall efficiency for the system of $12.6 \%$.

In order to understand this result it is useful to extract the effective solid angle subtended by a mirror $(0 \mathrm{~m})$ and compare it with a calculated result. Our measurement indicates that $\Omega_{m}=0.29 \times 2 \pi \mathrm{sr}$. The deviation of this number from $2 \pi$ is presumably attributable to loss of photons by obstructing objects (e.g., wires) and holes in the mirrors, absorption during reflection (reflectivity < 1 ), and beam quenching outside the region of sensitivity of the mirrors.

The solid angle lost to obstructions and holes can be calculated in a fairly straight-forward manner and is expected to be $\sim 0.113 \times 2 \pi \mathrm{sr}$. The net reflectivity of the mirrors should, on the average, be $\sim 0.70$. The resulting corrected value of $\mathrm{am}=0.53 \times 2 \pi$ sr suggests that about $1 / 2$ of the beam is quenched outside the region of sensitivity of the mirrors, which is considerably larger than we had anticipated. This issue could be investigated more thoroughly by using a small beam-defining aperture to determine the dependence of detector efficiency on distance from the beam axis.

G. A New Solenoid for the Hydrogen Atom Experiment

T.E. Chupp

Studies of atomic transition rates in the present H-parity apparatus (described in last year's annual report) 15 have shown that the the 570 Gauss axial magnetic field is not sufficiently uniform for the sensitive measurements 
of parity mixing in hydrogen. We have measured a transverse field (magnitude < 1 Gauss) which has a complicated shape and therefore cannot be cancelled in any simple fashion. This field gives rise to a complicated perpendicular electric field in the atoms ${ }^{-}$rest frame which produces spurious effects. Our study of instrumental effects and associated systematic errors indicates that we require a more uniform field, in direction and magnitude, than the present solenoid produces. We have therefore decided to design and construct a new magnet.

This section will describe the design strategy for the new solenoid and the status of construction. We have designed a system that will, in principle, produce a 570 Gauss axial field uniform to 0.1 Gauss over a $50 \mathrm{~cm}$ region with transverse components less than 0.005 Gauss. The axial field uniformity is due to the actual aximuthal currents in the windings while the transverse component arises from the radial and axial current components.

The current will flow through 20 pairs of 62 turn "pancakes" of anodized aluminum ribbon 0.031 " thick and 1.205" wide. The two pancakes in each pair are wound in opposite directions so that current flows from the outside to the inside in one coil, then from the inside to the outside in its partner. These pancakes will be wound on aluminum coil forms, separated by 0.005 " mylar for insulation, and will fit into our present system of vacuun can and flux return. This will be cooled by the present freon cooling system.

The magnetic field will be trimmed by controlling the current to certain pancake pairs. The two end pairs of pancakes which carry a greater current, will be driven by a separate power supply which will be in the "slave" mode while the remaining 18 pair of pancakes will be driven by a "master" supply. Shunting of individual pancake pairs will provide the fine trim. The main windings will require 23 amps while the upstream and downstream end coils will require 30.5 and 28.17 amps, respectively.

The radial currents which give rise to transverse field components, very nearly cancel for each pair since current flows in one and out in the adjacent pancake. The axial currents, however do not cancel since they flow at both the inside and at the outside of the coil. In order to approximately cancel these currents a loop of current is introduced, adjacent to but in the opposite direction. of unwanted axial current. Calculations show that the net transverse field are well below the specified level of 0.005 Gauss:

At the time of this writing the following progress has been made on construction of this new solenoid. The aluminum ribbon has been delivered from the mill and is in the process of being conditioned and anodized. The design of the coil windius assembly ae well as the necessary adaptors to our present system has been completed and their construction has begun.

H. Design and Testing of Prototype RF Cavities for the New Apparatus

E.G. Adelberger, D. Holmgren, and H. E. Swanson

The Mark II scheme requires two cavities each of. which produces uniform static and RF (1608 mHz) electric fields along the $B$ field ( $\hat{z})$ axis. 


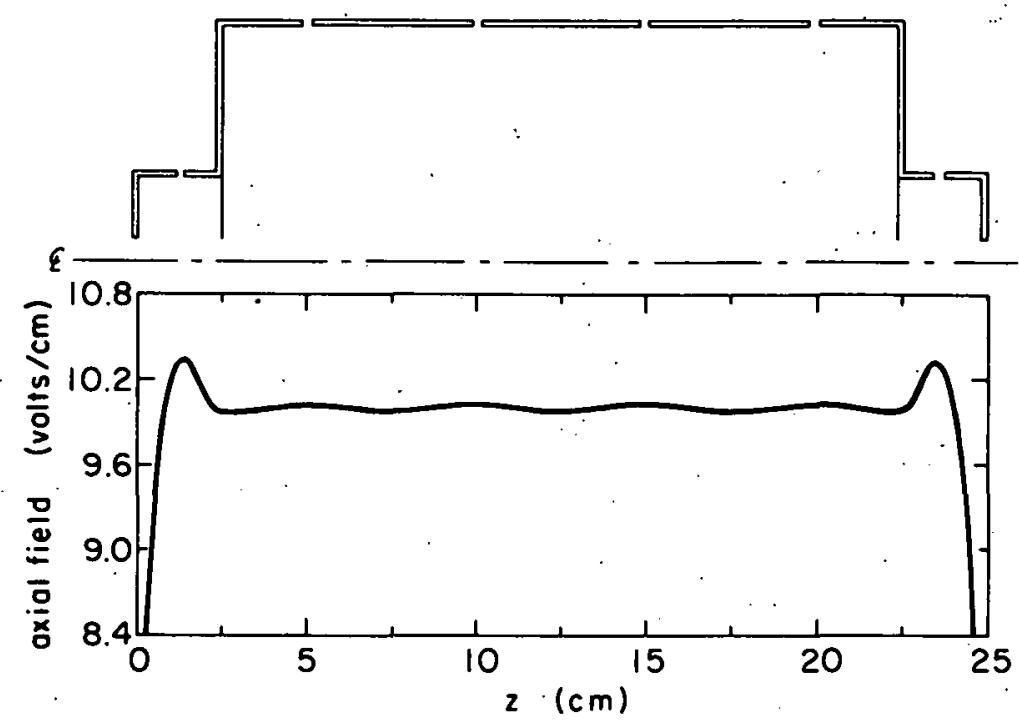

Fig. 2.3.H-1: Calculated axial electrostatic field in a $14 \mathrm{~cm}$ diameter cavity with $5 \mathrm{~cm}$ long segments; $5 \mathrm{~cm}$ diameter end caps are used to keep the static field uniform in the region of fringing $R F$ fields. The thin apertures $2.5 \mathrm{~cm}$ in from the ends terminate the RF fields.

Cylindrical cavities operating in the $\mathrm{TM}_{010}$ mode were chosen since. a cylindrically symetric apparatus suppresses false effects (see Section 2.3.C). This takes care of the RF fields: The static field along the $z$ axis can be generated by dividing the cavity into short. $(-5 \mathrm{~cm}$ long) cylindrical tubes and placing each section at a different static potential. This produces a very uniform field $E_{2}$ along the cavity axis (see Fig. 2.3.H-1 for the calculated fields). The difficulty arises in maintaining the $R F$ oscillations inside a segmented cavity since. the gaps between the segments interrupt the flow of the RF currents. We constructed two prototype cavities to test two different ideas for maintaining the RF currents.

1) cavity A with coaxial $\lambda / 4$ chokes

2) cavity $B$ with radial $\lambda / 4$ chokes

The cavities are indicated schematically in Fig. 2.3.H-2 where we show the three-segment front cavity. We experienced great difficulties with RF power "leaking out" of cavity A in spite of measures taken to "kill" the parasitic $\mathrm{TE}_{01}, \mathrm{TE}_{02}$, and $\mathrm{TE}_{03}$ modes which can propagate in the coaxial chokes.

On the other hand, cavity B works extremely well and radiates so little power from the slots that. unwanted coupling between the front and back cavities of the apparatus will not be a problem. "The concept has been so successful that we are designing the Mark II cavities using the scheme proven in the prototype. 

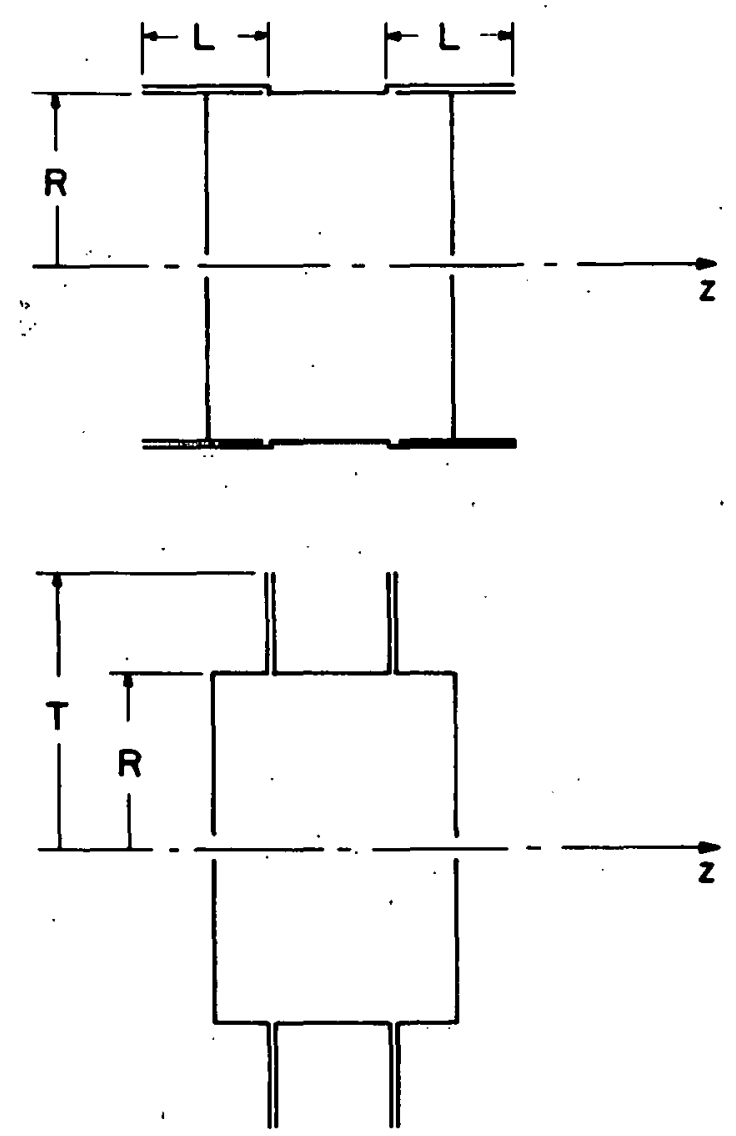

Fig. 2.3.H-2: Schematic drawings of two different schemes for segmenting the cavities

1) cavity with coaxial $\lambda / 4$ chokes

2) cavity with radial $\lambda / 4$ chokes in these cavities $\quad R=2.405(c / 2 \pi \nu)$

$\mathrm{L}=\lambda / 4=\mathrm{c} / 4 \mathrm{v}$ when $\nu=1608 \mathrm{mHz}$

$\mathrm{T}=3.832(\mathrm{c} / 2 \pi \mathrm{v})$

I. Programmable NMR B Field Regulator with IEEE Interface

D.A. Peterson and H.E. Swanson

A proton MMR B field regulator is under construction for the H-Atom solenoid. When in operation the control computer directs the microprocessor based B field controller to a new field and senses when it has been achieved. The NMR consists of a water sample placed in the coil of a tuned circuit. This tank circuit is weakly coupled to a sustaining RF oscillator. The sample is 
placed in an external B field whose direction is perpendicular to the RF $B$ field of the coil. When external $B$ is such that the proton Larmour precision frequency is equal to the RF frequency transitions between the spin states occur. Since the lower energy states have the greater population at room temperature, energy is taken from the field. This has the effect of lowering the tank circuit $Q$ at the proton resonance and can be observed as a decrease in the RF amplitude measured across the coil. Generally the $B$ field is swept across the resonance using Helmholtz coils placed about the sample and the resulting $\mathrm{RF}$ amplitude dips correlated with the modulation to obtain a $B$ field error signal.

To some extent however, this perturbs the external field in the vicinity of the sample. Since it is desirable to have the probe close to the beam position, we chose to sweep the RF frequency about the resonance avoiding the Helmholtz coils.

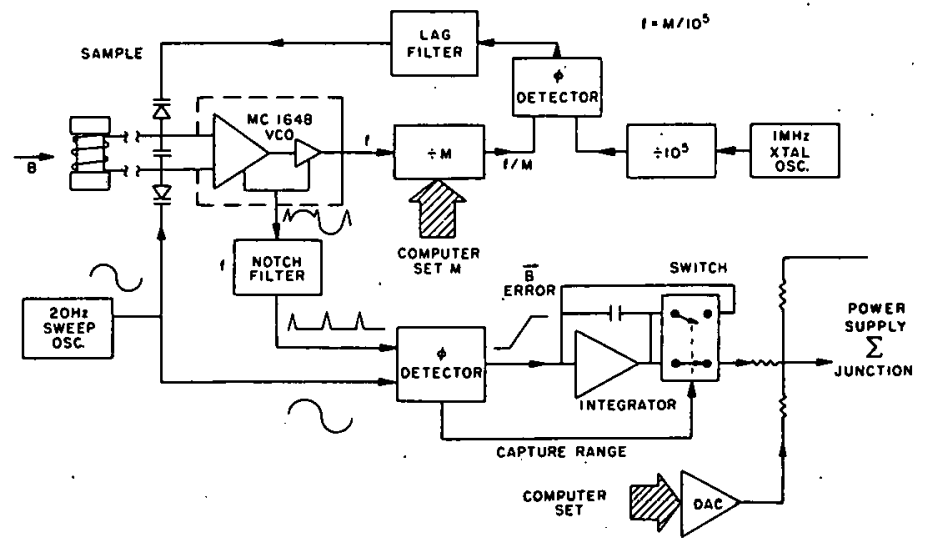

Fig. 2.3.I-1: NMR B field regulator

A block diagram of the regulator circuit is shown in Fig. 2.3.I-1. There are two control 10ops; one which locks the frequency to a multiple of a stable crystal reference and another which locks the B field to the phase of the NMR dips with respect to the modulation. The sustaining oscillator is a Motorola MC 1648 VCO typically used in phase locked loop applications. The tuned circuit consists of a coil wound on the sample vial and shunt capacitors including two voltage variable capacitors (varicaps) for frequency control. A significant amount of the capacitance comes from the coax cable used to separate the sample from the rest of the circuit parts, some of which are magnetic and therefore cannot be near the probe. 
Discussing first the frequency loop, the VCO output is divided by $M$ using scaler chips to count to $M$ and outputting a pulse when this occurs. Similarly the $1 \mathrm{mHz}$ crystal oscillator output is divided by $10^{5}$. The VCo will typically run at about $2.5 \mathrm{mHz}$, thus 5 decimal digit resolution can be obtained. in the quantity $M$ with settling times of the order of a second. Both outputs go to a phase detector whose filtered output is applied to one of the varicaps. The circuit seeks the frequency $f$ such that the average phase error of the two signals is zero. The $\mathrm{VCO}^{-} \mathrm{s}$ frequency is thus stabilized to $\mathrm{M} / 10^{5} \mathrm{~Hz}$. $\mathrm{M}$ is loaded by the microprocessor and is calculated from the desired $B$ field.

The circuitry within the MC 1648 includes an RF detector and automatic gain control circuit (AGC) to hold the oscillator amplitude below saturation (for spectral purity) and constant regardless of the frequency. fortunately, this AGC signal is brought out of the package and can be used as a measure of the power required to sustain the oscillation. Thus as the frequency is swept across the proton resonance, the dip in the tuned circuit $Q$ is reflected in this signal. The frequency is modulated by applying a $20 \mathrm{~Hz}$ sinusoidal signal to the other varicap. A disadvantage in frequency as opposed to B fleld modulation is that the fundamental appears on the AGC signal. The tank circuit $Q$ is proportional to $\sqrt{\mathrm{L} / \mathrm{C}}$ and for small modulations, a change $C$ makes a proportional change in $Q$ and a corresponding change in the AGC signal.

To remove this fundamental, the amplified AGC signal is passed through a notch filter tuned to $20 \mathrm{~Hz}$. The filtered MMR dips are phase correlated with the modulation signal to obtain the B field error signal, e.g., if the NMR dips occur when the modulation signal was passing through zero, the B field corresponds to the oscillator frequency and no error exists. The phase detector signals that the $B$ field is within the capture range of the system by the presence of NM dips. The function of the integrator is best explained with a description of system operation.

To begin, the controller loads $M$ to determine the frequency. It then sets. the magnet current by loading the calculated DAC value into its register where this is chosen to be slightly less than the required current. At this point the integrator is isolated from the power suppiy so the magnet current corresponds to the DAC reference. The DAC register is then incremented until the presence of NMR dips indicates capture can be achieved. The integration capacitor is unshorted and the integrator output is connected to the summing junction. Neglecting leakage acioss the switch, the output of the integrator seeks zero average $B$ field error which is the desired result. Transient loss of lock merely returns the power supply reference to the DAC to begin. a ner search for capture. 
J. RF Phase Shift Electronics

T.E. Chupp, D.A. Peterson, and H.E. Swanson

The $1608 \mathrm{MHz}$ microwave source described in last year's progress report has been modified to drive two cavities with a relative phase variable between 0 and 360 degrees. Power from the oscillator feeds a "two-way splitter" whose outputs form phase correlated sources each with approximately $50 \%$ of the oscillators power. These are isolated using ferrite isolators and independently power leveled in the manner previously discussed. Included in one of the channels are two phase shifters purchased frow Vectronics Microwave Corporation. One of these is a four quadrant shifter requiring two logic signals to select $00,90^{\circ}$, $180^{\circ}$, and $270^{\circ}$ shift. The other is an analog device which translates a voltage signal into a phase shift in the range 0 to $100^{\circ}$. The combination of the two units allows us to set the relative phase anywhere within $2 \pi$ radius and accurately modulate the phase by $\pi$ radians.

\section{K. H-Atom Signal Normalizer}

\section{H.E. Swanson}

An analog signal normalizer was designed and built. It has two signa? inputs $A$ and $B$ and removes beam fluctuations, common to both inputs, from the $B$ signal. The unit has the following transfer function

$$
v_{\text {out }}=\frac{K_{1} A-K_{2} B}{K_{3} A}
$$

where the gains $\mathrm{K}_{1}, \mathrm{~K}_{2}$ and $\mathrm{K}_{3}$ are individually adjustable. It is assumed that $B$

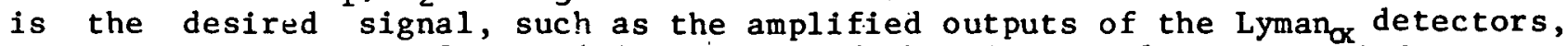
which measure the $\alpha->\beta$ transition rate, and the $A$ signal is an independent monitor of the metastable beam current.

Discriminators monitor the input signal levels and switch to default values previously set to nominal signal values. Thus saturation of the data acquisition system is prevented in the event of an ion source spark or other beam interruption.

\section{References}

1. R.R. Lewis and W.L. Willians, Phys. Lett. 59B, 70 (1975).

2. L.L. Lewis et al., Phys. Rev. Lett. $39,7 \overline{95}$ (1977).

3. P. Baird et al., Phys. Rev. Lett. 39, 798 (1977).

4. L.M. Barkov and M.S. Zolotoryov, JET $\bar{P}$ Lett. 27, 379 (1978).

5. R. Conti et a1., Phys. Rev. Lett. 42, 343 (1979).

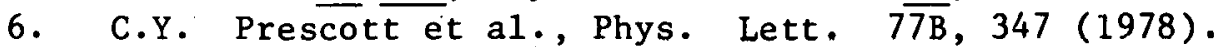

7. W.J. Marciano and A.I. Sanda, Phys. Rev. D 17, 1313 (1978). 
8. L. Wolfenstein, Phys, Rev. D 19, 3450 (1979).

9. E.G. Adelberger, T.A. Trainor, and E.N. Fortson, Bull. Am. Phys. Soc. 23, 546 (1978).

10. R.W. Dunford, R.R. Lewis, and W.L. Williams, Phys. Rev. Al3, 2421 (1978).

11. E.A. Hinds and V.W. Hughes, Phys. Lett. 67B, 487 (1977) and E.A. Hinds, private communication, 1979.

12. Our code is an adaptation of one described in G.G. Ohlson and J.L. McKibben, Los Alamos report LA-3725 (1967) unpublished: (We are indebted to W.L. Williams for informing us of an error in the Los Alamos code.) i-5;13. R.D. Deslattes (private communication), 1979.

14. E.A. Hinds, Phÿs. Kev. Lett. 44, 374 (1980).

15. Nuclear Physics Laboratory Annual Report, University of Washington (1979), p. 26 . 


\section{NUCLEAR STRUCTURE AND REACTIONS}

1. Gamow-Teller Giant Resonances in the $\beta^{+}$Decay of $T=T_{3}=3 / 2$ Nuclei

A. Introduction

\section{E.G. Adelberger}

At present very little is known about the strength function for spin flip transitions. The simplest such transitions are those induced by the Gamow-Teller (GT) operator. Evidence for giant GT resonances has been inferred from a number of sources including the hindrance of low lying beta transitions, bumps in $(p, n)$ spectra, and in the strength function of delayed neutron and proton emission. The beta-plus decays of proton rich higher isospin states in light and medium mass nuclel can provide unambiguous measurements of the GT strength over a range of excitation energies wide enough to include the expected location of the GT giant resonance. Consider for example the decays of a $\mathrm{T}=\mathrm{T}_{3}=(\mathrm{Z}-\mathrm{N}) / 2=3 / 2$ nucleus. The Coulomb displacement energy increases the mass of the parent so that $\beta^{+}$decays are energetically allowed up to an energy which lies considerably above the analog state. One expects that the $\beta^{+}$decays will primarily feed the $\mathrm{T}_{<}$giant GT resonance built upon the analog state. This is expected to lie at roughly the energy of the analog state (the $T_{<} G T$ resonance has its energy depressed by the isospin splitting).

- The $\beta^{+}$decays of $T_{3}=3 / 2$ nuclei have been studied in a beautiful series of experiments at Berkeley. The LBL researchers obtain proton spectra following the decay of the $\mathrm{T}=\mathrm{T}_{3}=3 / 2$ parent to proton unstable states in the $\mathrm{T}_{3}=1 / 2$ daughter. To infer a $\beta^{+}$decay scheme from the delayed proton spectra one needs to know the spins, parities and proton branching ratios of states in the daughter nucleus. This is illustrated in Fig. 3.1-1, where we show the situation for the decays of ${ }^{25} \mathrm{Si}$.

Fig. 3.1-1: The $\beta^{+}$-decay of ${ }^{25} \mathrm{Si}$.

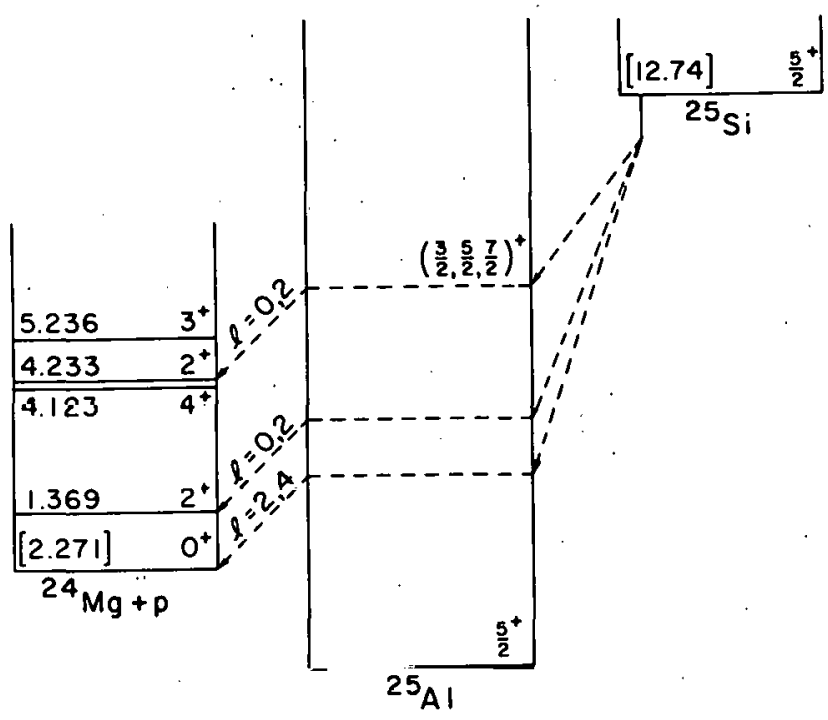


A given proton group may result from several possibilities--decays of the ${ }^{25} \mathrm{Al}$ daughter to the ground state or to excited states of ${ }^{24} \mathrm{Mg}+\mathrm{p}$. Clearly a delayed proton group with a given intensity corresponds to a much larger GT matrix element if it arises from decays feeding excited states of $24 \mathrm{Mg}$ than if it arises from ground state decays. Also note that one expects levels fed in the $\beta^{+}$decays to emit protons to excited states of ${ }^{24} \mathrm{Mg}$ since allowed $\beta^{+}$ transitions can have $\ell=0$ proton decays to excited states of $24 \mathrm{Mg}$ but only $\ell=$ 2 or $l=4$ proton decays to the $24 \mathrm{Mg}$ ground state.

We have begun careful study of the spectroscopy of the $\beta^{+}$daughters beginning with ${ }^{25} \mathrm{Al}$ and ${ }^{29} \mathrm{P}$. We obtained thin target excitation functions for polarized proton scattering at $l \mathrm{ab}$ angles ranging between $55^{\circ}$ and $155^{\circ}$. The method has been described in last year's annual report.

B. Spectroscopy of ${ }^{29} \mathrm{P}$ and the Giant Gamow-Teller Resonance in the $\beta^{+}$
Decay of ${ }^{29} \mathrm{~S}$

E.G. Adelberger, C.D. Hoyle, P.G. Tkossi, and K.A. Snover

We have continued our investigation ${ }^{1}$ of the spectroscopy of ${ }^{29} \mathrm{P}$ in order to interpret the Berkeley delayed proton spectra ${ }^{2}$ from the $\beta^{+}$decay of $29 \mathrm{~S}$. In order to extract a decay scheme from the proton spectrum following the $\beta^{+}$decay of $29 \mathrm{~S}$ one needs to know whether a given delayed proton group corresponds to the proton decay of a ${ }^{29} \mathrm{P}$ level to the ground state or an excited state of ${ }^{28} \mathrm{Si}$. We used the ${ }^{28} \mathrm{Si}\left(\mathrm{p}, \mathrm{p}_{\mathrm{o}}\right)$ and ${ }^{28} \mathrm{Si}\left(\mathrm{p}, \mathrm{p}_{1}\right)$ reactions to identify the spins, parities, excitation energies and partial widths of states in ${ }^{29} \mathrm{P}$ covering a region from $\mathrm{E}_{\mathrm{x}}=5.3$ to $\mathrm{E}_{\mathrm{x}}=10.1 \mathrm{MeV}\left(\mathrm{E}_{\mathrm{p}}=2.6\right.$ to $\left.\mathrm{E}_{\mathrm{p}}=7.9 \mathrm{MeV}\right)$. We searched for levels which can be fed in the allowed $\beta^{+}$decay of ${ }^{29} \mathrm{~S}-\mathrm{-i} . \mathrm{e}$., levels with $\mathrm{J}^{\mathrm{N}} \cdot=3 / 2^{+}$, $5 / 2^{+}$or $7 / 2^{+}$, having excitation energies and values of $\Gamma_{\mathrm{p}_{1}} / \Gamma_{\mathrm{p}_{\mathrm{o}}}$ consistent with the assignment of a given delayed proton group to ground state or excited state decays. Many of the $\beta^{+}$branches proposed in Ref. 2 are consistent with our results. However we found that in several cases proton groups, assumed in Ref. 2 to arise from ground state decays, in fact arise from decays to excited states of ${ }^{28} \mathrm{Si}$. This makes a pronounced change in the shape of the $\beta^{+}$strength function--clustering the Gamow-Teller strength in a compact region as discussed below.

Thin target excitation functions for ${ }^{28} \mathrm{Si}+\mathrm{p}$ were obtained at a number of angles at bombarding energies between 2.6 and $7.9 \mathrm{MeV}$. An example of this data 10 chown in $\mathrm{Fig}$. 3.1-2. In the following paragraphe we diccues thoce caces where our results are inconsistent with the decay scheme of Ref. 2 and our resolution of the inconsistencies. 


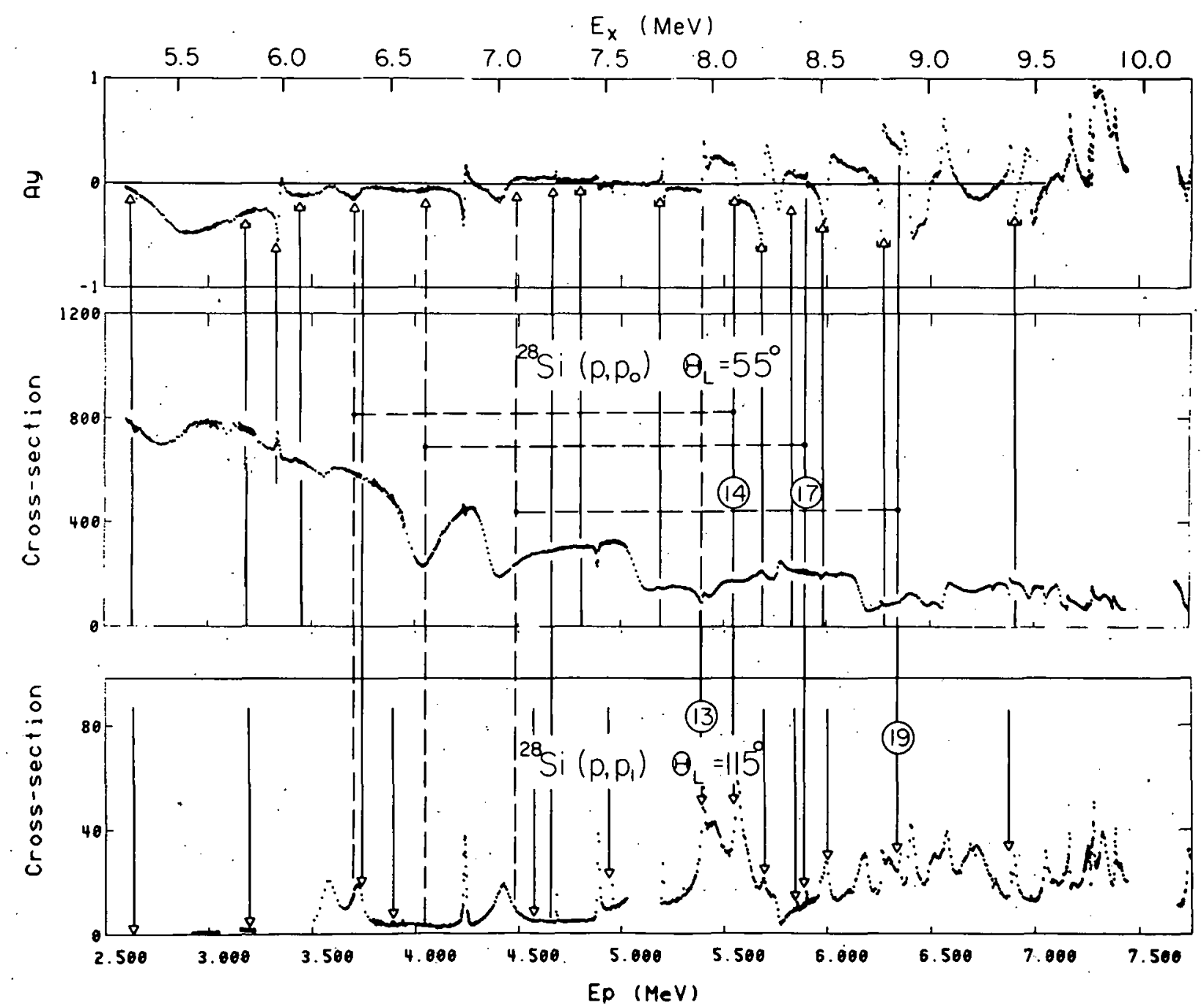

Fig. 3.1-2: Upper Part: cross sections and analyzing powers for elastic proton scattering from ${ }^{28} \mathrm{Si}$ at $\theta_{\mathrm{LAB}}=55^{\circ}$. Lower Part: cross sections at $\theta_{\mathrm{LAB}}=$ $115^{\circ}$ for the ${ }^{28} \mathrm{Si}\left(\mathrm{p}, \mathrm{p}_{1}\right)$ reaction. The solid vertical lines mark the location of the groups observed in the LBL experiment. Dotted Iines labeled with the peak numbers of the LBL work are moved to. higher excitation energies as discussed in the text. 


\section{1) The 6.33 and $8.11 \mathrm{MeV}$ Levels}

Groups 15 and 25 of Ref. 2 were assigned to the $p_{1}$ and $p_{0}$ decays of the $8.24 \mathrm{MeV}$ state $\left(\mathrm{E}_{\mathrm{p}}=5.67 \mathrm{MeV}\right)$ yielding a $10 \mathrm{~g} \mathrm{ft}$ of 4.61 . Group 24 was assigned to the $p_{0}$ decays of a state at $E_{x}=8.11 \mathrm{McV}\left(E_{p}=5.50 \mathrm{MeV}\right)$ with a corresponding $\log \mathrm{ft}=4.83$. However, we find that the resonance in the inelastic cross-section corresponding to the $8.11 \mathrm{MeV}$ state is much stronger than the one corresponding to the $8.24 \mathrm{MeV}$ state. (Preliminary analysis of our data at $55^{\circ}$ indicates $\Gamma_{\mathrm{p} 1} / \Gamma_{\mathrm{p}} \quad 1.4$ for the $8.11 \mathrm{MeV}$ state and 0.3 for the 8.24 MeV state.)

Peak 14 was assigned in Ref. 2 to the $p_{0}$ decay of the $E_{x}-6.33 \mathrm{MeV}$ state but no candidate for the $\Gamma_{p_{1}}$ decay was proposed although the $6.33 \mathrm{MeV}$ level desays $80 \%$ of the lime ${ }^{3}$ ro the first excited state of ${ }^{28} \mathrm{Si}$. While peak 4 (assigned in Ref. 2 to the $p_{1}$ decay of a postulated new state at $6.36 \mathrm{MeV}$ ) could correspond to the $p_{1}$ branch of the $6.33 \mathrm{MeV}$ state it is too weak to form a pair witli gluup 14. 'I'hus group 1/ was misasilgned in the Berkeley work. Consistency between the Berkeley data and our scattering data can be achieved if group 14 is assigned to the $\mathrm{p}_{1}$ decay of the $8.11 \mathrm{MeV}$ state. Using the observed branches of Ref. 2 we then modify the $\log \mathrm{ft}$ values for both the 6.33 and 8.11 $\mathrm{MeV}$ states to 5.74 and 4.49 , respectively.

2) The 6.65 and 8.43 states

Group 17 was assigned in Ref. 2 to the $p_{0}$ decay of a proposed new state at $E_{X}=6.653 \mathrm{MeV}$. We searched for this state unsuccessfully. However, at $E_{X}=$ 8.43 ( $1.78 \mathrm{MeV}$ higher) we find a narrow state with $\mathrm{J} \pi=5 / 2^{+}$which decays via both the $p_{0}$ and $p_{1}$ channels. We therefore conclude that group 17 is due to the $\mathrm{p}_{1}$ decay of this state. The close proximity of this new level to the $\mathrm{T}=3 / 2$ analog would make it difficult to see the $p_{0}$ branch in the Berkeley experiment. Based on the intensity of group 17 we then estimate $\log \mathrm{ft}<4.68$ for the 8.43 $\mathrm{MeV}$ state.

3) The 7.08 and $8.86 \mathrm{MeV}$ States

Group 19 was assigned in the Berkeley work to a "new" state at $7.083 \mathrm{MeV}$. We were unable to locate this state as a resonance. A better candidate for this group is the $\mathrm{P}_{1}$ decay of the state at $8.862 \mathrm{MeV}$ which we see as a $\mathrm{p}_{0}$ and $\mathrm{p}_{1}$ resonance. Our measurements indicate $\mathrm{J}^{\pi}=3 / 2^{+}$for the $8.862 \mathrm{MeV}$ state which overrides the previous $1 / 2^{+}$assignment. Then $\log \mathrm{ft}=4.91$ for this state.

With these modifications we can reconcile our scattering data with the Berkeley data at excitation energies above $7.5 \mathrm{MeV}$. At lower energies our data, itself, does not account for all the levels inferred from the delayed proton data. The only previously unobserved state postulated in the Berkeley work that we could confirm is the $\mathrm{E}_{\mathrm{X}}=6.505 \mathrm{MeV}$ level where a narrow resonance in the ${ }^{28} \mathrm{Si}\left(\mathrm{p}, \mathrm{p}_{1}\right)$ excitation function can be seen. We searched and were unable to observe as resonances the states at $E_{X}=5.293 \mathrm{MeV}$ (groups 1 and 7), $5.826 \mathrm{MeV}$ $(10,3), 6.074 \mathrm{MeV}(12), 7.148 \mathrm{MeV}(8)$, and $7.384 \mathrm{MeV}(21)$. The presence of: 
Table 3.1-1:

\begin{tabular}{|c|c|c|c|}
\hline$E_{p}(\mathrm{MeV})$ & $E_{x}(\mathrm{MeV})$ & $\begin{array}{l}\operatorname{Ref}_{\mathrm{J} \pi} \\
\end{array}$ & $\begin{array}{c}\text { Present Work } \\
\mathrm{J} \pi\end{array}$ \\
\hline 4.228 & 6.828 & $5 / 2^{+}$ & $3 / 2^{+}$ \\
\hline $\begin{array}{l}4.688 \\
4.579\end{array}$ & 7.272 & $712-$ & $5 / 2^{+}$ \\
\hline 4.948 & 7.523 & $\left(1 / 2^{-}\right)$ & $3 / 2^{+}$ \\
\hline $\begin{array}{l}5.188 \\
5.550\end{array}$ & $\begin{array}{l}7.755 \\
8.204\end{array}$ & $\begin{array}{l}3 / 2^{+} \\
(3 / 2,5 / 2)^{+}\end{array}$ & $\begin{array}{l}5 / 2^{+} \\
5 / 2^{+}\end{array}$ \\
\hline 5.670 & 8.220 & $(3 / 2,5 / 2)^{+}$ & $3 / 2^{+}$ \\
\hline $\begin{array}{l}5.750 \\
5.890 *\end{array}$ & $\begin{array}{l}8.297 \\
8.432\end{array}$ & $(1 / 2,3 / 2)^{-}$ & $\begin{array}{l}(1 / 2)^{-} \\
5 / 2^{+}\end{array}$ \\
\hline 5.990 & 8.529 & $(3 / 2,5 / 2)^{+}$ & . $3 / 2^{+}$ \\
\hline $\begin{array}{l}6.160 \\
6.340\end{array}$ & $\begin{array}{l}8.693 \\
8.867\end{array}$ & $\begin{array}{l}1 / 2^{+} \\
1 / 2^{+}\end{array}$ & $\begin{array}{l}3 / 2^{+} \\
3 / 2^{+}\end{array}$ \\
\hline $\begin{array}{l}6.390 \\
6.480 \\
6.880\end{array}$ & $\begin{array}{l}8.915 \\
9.002 \\
9.388\end{array}$ & $\begin{array}{l}(3 / 2,5 / 2)^{+} \\
(+)\end{array}$ & $\begin{array}{c}5 / 2^{+} \\
(5 / 2)^{+} \\
3 / 2^{+}\end{array}$ \\
\hline
\end{tabular}

*The excitation energy of this state is from our work.

Fig. 3.1-3: Energy-distribution of the GR strength for the $\beta^{+}$decay of ${ }^{29} \mathrm{Sj}$. Dotted lines are used to indicate the. LBL interpretation when different from the results of this study.

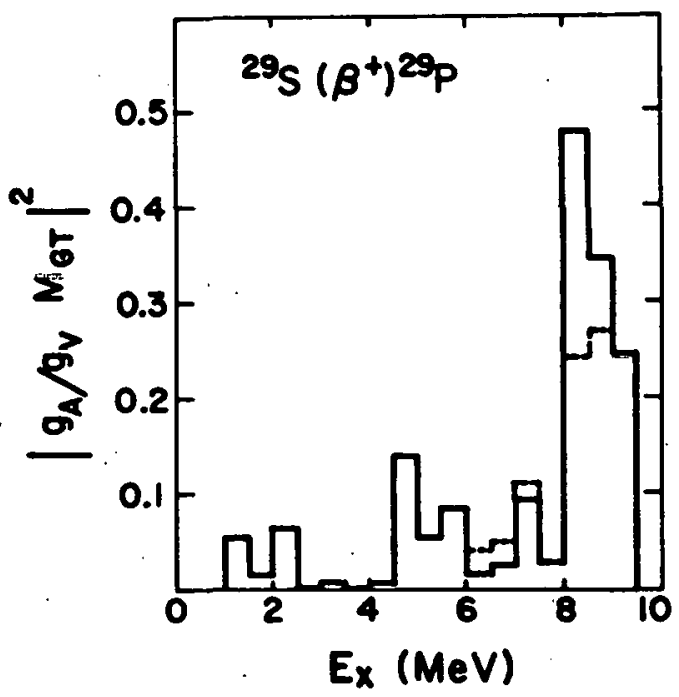


the first two of these states is well established (see Ref. 2 and references therein). Our inability to observe them merely indicates that the total width of the resonance is less than $1 \mathrm{keV}$. At this time we find no alternative candidates for groups 12,8 and 21 .

In Fig. 3.1-3 we show the distribution of the Gamow-Teller strength as a function of energy. The dotted line represents the Berkeley interpretation and the solid line the same strength corrected on the basis of the arguments above.

Further resonance analysis of our data, now in progress, w111 provide more accurate values of the branching ratios to the ground and first excited state and thus set further quantitative restrictions on the interpretation of the Berkeley experiment. This analysis will also yield spin and parities for the stronger of the $p_{0}$ resonances. In Table 3.1-1 we sumarize oome tentative splu assignments and compare with the values 1 isted in the latest compllation. ${ }^{3}$ In making these assignments for the negative parity state we are assuming that the $l$-value of the resonances is correctly given in. the literature. The behavior of the analyzing power at $55^{\circ}$ then selects the $J^{\pi}$ value:

C. JT Assignments in ${ }^{25} \mathrm{Al}$ and the Glant Gamow-Teller Resonance in the
$\beta^{+}$Decay of ${ }^{25} \mathrm{Si}$

E.G. Adelberger, C.D. Hoyle, P.G. Ikossi, and K.A. Snover

In order to study the Gamow-Teller Giant Resonance in the $\beta^{+}$decay of ${ }^{25} \mathrm{Si}$, one needs a decay scheme for ${ }^{25} \mathrm{Si}$ and the $J \pi$ values of the states in $25 \mathrm{Al}$ fed by the $\beta^{+}$decay. Previous decay schemes and $J^{\pi}$ assignments 3,4 contain some ambiguities. To resolve these ambiguities, we have begun a study of the ${ }^{24} \mathrm{Mg}(\mathrm{p}, \mathrm{p})$ reaction induced by polarized protons.

We have measured cross sections and analyzing powers with good energy resolution for the $24 \mathrm{Mg}\left(\mathrm{p}, \mathrm{p}_{\mathrm{O}}\right)$ reaction at $\mathrm{lab}$ angles of $53^{\circ}, 68^{\circ}, 83^{\circ}, 113^{\circ}$, 1380 , and $153^{\circ}$. Incident proton lab energies ranged from approximately $2.3 \mathrm{MeV}$ to $7.3 \mathrm{MeV}$. At most of these energies we also obtained cross section and aralyzing power data for the $24 \mathrm{Mg}\left(\mathrm{p}, \mathrm{p}_{1}\right)$ reaction (see Fig. 3.1-4). The targets had $24 \mathrm{Mg}$ contents ranging from about $10 \mu \mathrm{g} / \mathrm{cm}^{2}$ to about $30 \mu \mathrm{g} / \mathrm{cm}^{2}$. Targets were prepared by evaporating $1 \mu \mathrm{g} / \mathrm{cm}$ of gold onto $5 \mu \mathrm{g} / \mathrm{cm}$ carbon backings. Then without breaking the bell jar vacuum natural magnesium was evaporated onto the gold. The gold was used since $\mathrm{Mg}$ adheres to gold much better than it does to carbon. The gold also provided a convenient method for obtaining the relative normalization of the different detectors since the scattering from the gold is Rutherford. The absolute normalization was obtained from an angular distribution at $2.60 \mathrm{MeV}$. The polarized ion source was operated in the fast spin flip mode with a beam current of $\sim 40 \mathrm{nA}$ and polarization of $\sim 0.75$. The counting time for each point was $\sim 5$ minutes. 
The off line analysis of the data was done with the program DMULTS. DMULTS is a peak summing program written for the PDP $11 / 60$ which uses kinematics to obtain an energy calibration of each spectrum. This calibration is then used to shift the peak windows in each spectrum automatically with each energy change. This permits a large number of runs to be summed with a minimum of operator effort. Background subtraction was done by fitting a straight line to the peak-free region of each spectrum and then subtracting the appropriate area from each peak.

A resonance analysis of the data has not yet been performed, but by comparing the elastic scattering data to theoretically calculated shapes, 1 it appears that some of the previous $\mathrm{J} \pi$ assignments are incorrect and some tentative new assignments can be made (see Table 3.1-2). A preliminary look at the inelastic data indicate probable errors in previous decay schemes. The new data indicates, that in some of the $\beta^{+}$decays of ${ }^{25} \mathrm{Si}$, the ${ }^{25} \mathrm{Al}$ * daughter is at a higher excitation energy than was previously assigned (see Fig. 3.1-4). Reassigning the decays to higher states in $25 \mathrm{Al}$ puts more of the decay strength in the region of the expected Gamow-Teller resonance. With a more careful analysis of the data, we will be able to make more definitive statements about new $J \pi$ values and new decay schemes.

Table 3.1-2: Previous $J^{\pi}$ values and $J^{\pi}$ values from our data

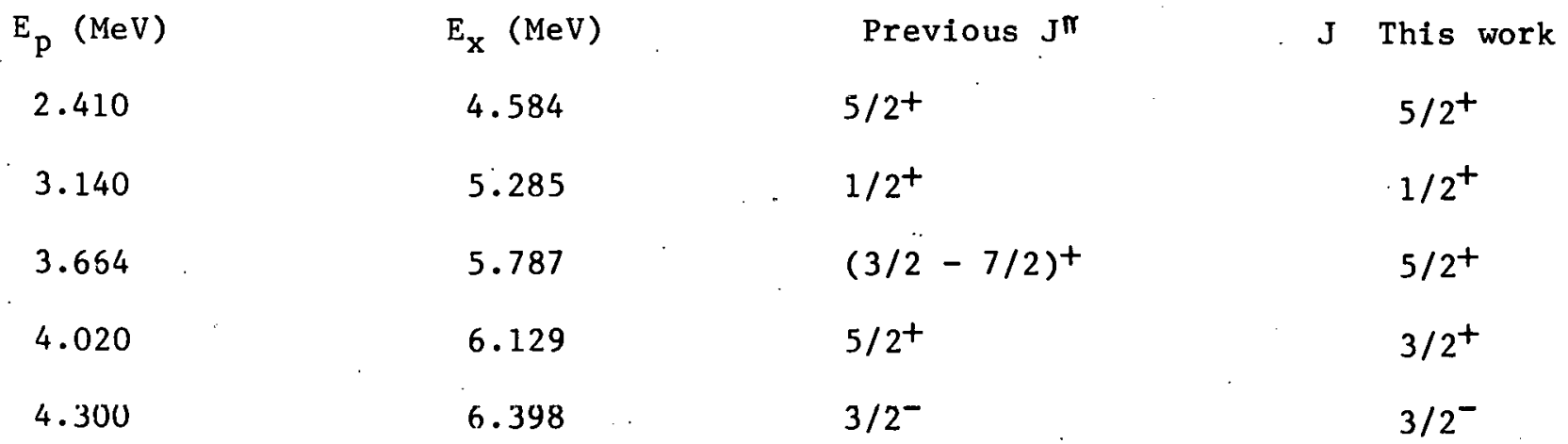

\section{References}

1. Nuclear Physics Laboratory Annual Report, University of Washington (1979), P. 47.

2. D.J. Vieira, R.A. Gough, and J. Cerny, Phys. Rev. C19, 177 (1979).

3. Endt and Van der Leun, Nucl. Phys. A310, 1 (1978).

4. R.G. Sextro, Ph.D. thesis (unpublished), University of California, Berkeley, 1973. 


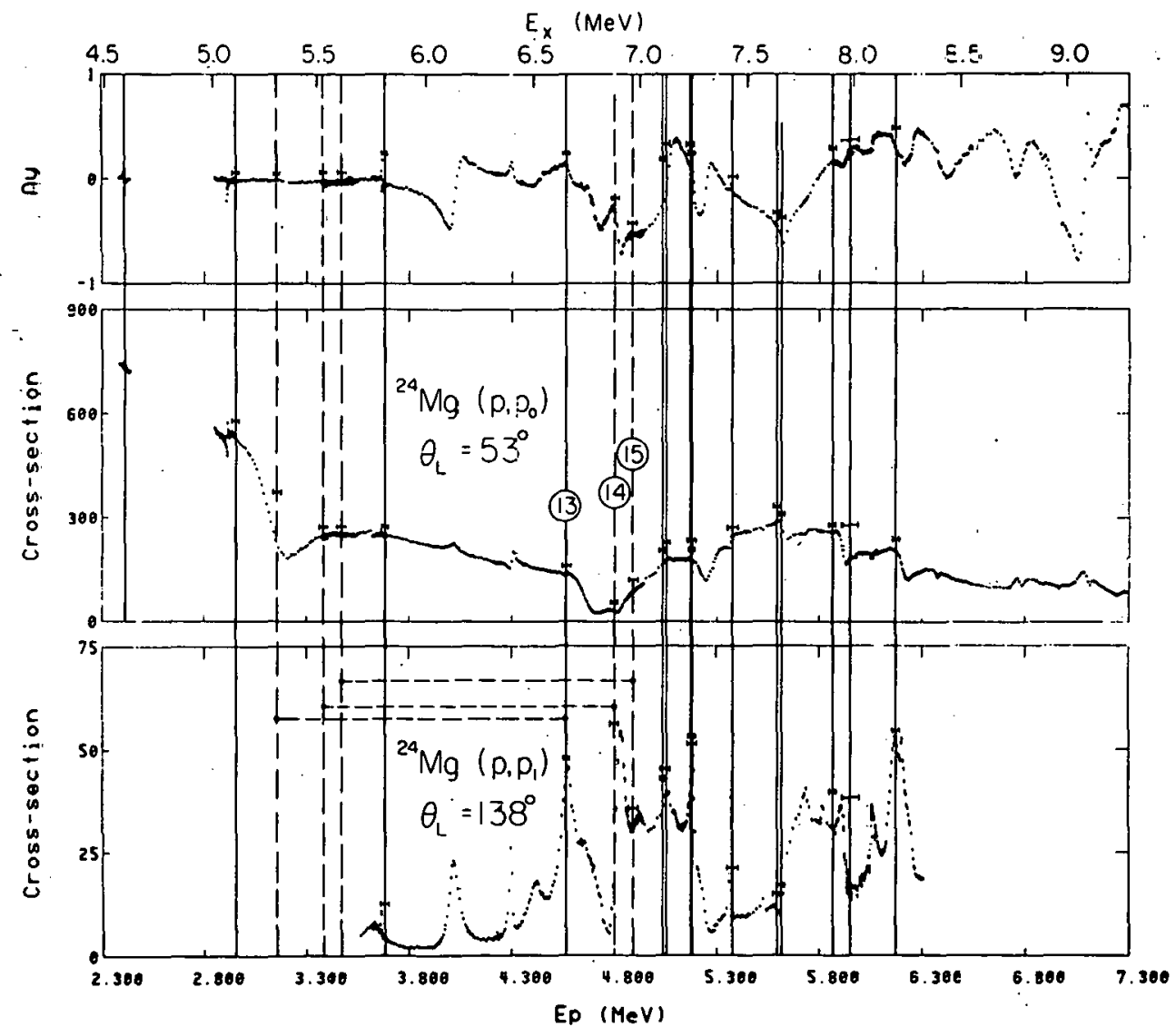

Fig. 3.1-4: The unlabeled vertical lines correspond to energies where we should see a resonance if the beta delayed proton assignments in Ref. 4 are correct. Peaks 13, 14, and 15 in Ref. 4 were assigned as Po decays. The vertical lines lateled 13, 14, and 15 correspond to the energ-es wiere we should see a resonance if these peaks were $\mathrm{p}_{1}$ decays and not $\mathrm{p}_{0}$ decays. The dashed vertical lires connected to the lines labeled 13, 14, and 15 by a horizontal line, shcw where we should see a resonance if peaks 13,14 , and 15 were Po decays. For these three peaks, we do not see resonances of the appropriate spin at the appropriate energy for a po decay. Therefoze we reassign them as $\mathrm{P}_{1}$ decays. 


\section{2. $\beta-\gamma$ Circular Polarization Correlation in the $\beta^{+}$Decay of ${ }^{24} \mathrm{Al}$}

E.G. Adelberger, C.D. Hoyle, and K.A. Snover

Fig. 3.2-1: Decay scheme for isospin forbidden Fermi transitions.

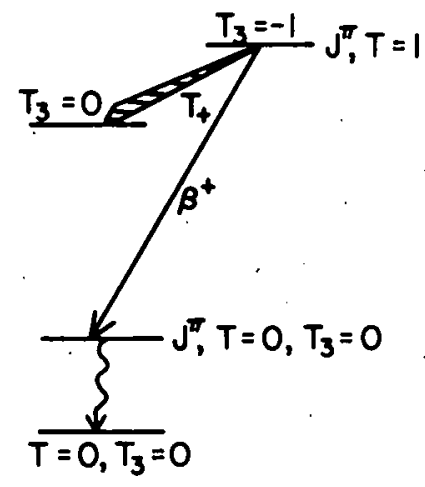

Decay scheme for isospin forbidden transitions.

If isospin is a good quantum number, Fermi transitions between states of different isospin are forbidden. We have begun a study of the $\beta-\gamma$ circular polarization correlation in the $\beta^{+}$decay of ${ }^{24} \mathrm{Al}$ which will permit us to measure the isospin forbidden Fermi matrix elements connecting the lowest $4^{+} \mathrm{T}=1$ state in $24 \mathrm{Mg}$ with several $4^{+} \mathrm{T}=0$ levels. Since we expect large analog-anti-analog isospin mixing matrix elements in selfconjugate nuclei ${ }^{1}$, we hope to use the measured isospin mixing matrix elements to probe the strength distribution of the anti-analog configuration.

Consider the simple situation in Fig. 3.2-1 with the initial state $\left.|i\rangle=J \pi, T=1, T_{3}=1\right\rangle$ and final state $|f\rangle=\left|J^{\pi} T=0, T_{3}=0\right\rangle+\alpha_{T}\left|J^{\pi}, T=1, T_{3}=0\right\rangle$. Using the well known relations

$$
f t=\frac{k}{G_{V}^{2} M_{F}^{2}+G_{A}^{2} M_{G T}^{2}}
$$

where

$$
K=\frac{2^{3}(\ln 2)^{7}}{m_{0} c^{4}}
$$


$\mathrm{G}_{\mathrm{V}}=$ weak interaction vector coupling constant

$\mathrm{G}_{\mathrm{A}}=$ weak interaction axial vector coupling constant

$M_{F}=\left\langle f\left|\sum_{f}(j)\right| i\right\rangle=\left\langle f\left|T_{+}\right| i\right\rangle$

$M_{G T}=\left\langle f \sum_{j} t_{+}(j) \quad \vec{\sigma}(j) \mid i\right\rangle$

and

$$
\begin{aligned}
M_{F} & =\left\langle T=0, T_{3}=0\left|T_{+}\right| T=1, T_{3}=-1\right\rangle+\alpha_{T}\left\langle T=T=1, T_{3}=0\left|T_{+}\right| T=1, T_{3}=-1\right\rangle \\
& =\alpha_{T} \sqrt{2}
\end{aligned}
$$

we see that, in order to measure the Fermi matrix elements $M_{F}$ and the isospin mixing amplitude $\alpha_{\mathrm{T}}$, we need to measure a quantity that depends on the relative size and phase of the Fermi and famon-Toller matrix olcmento. If we define $y=$ $\mathrm{G}_{\mathrm{V}} \mathrm{M}_{\mathrm{F}} / \mathrm{G}_{\mathrm{A}} \mathrm{M}_{\mathrm{GT}}$, equation (1) becomes

$$
f \tau=\frac{K}{G_{V}^{2} M_{F}^{2}} \frac{y}{1+y^{2}}
$$

Then $y$, along with the known ft values will yield the Fermi matrix element. For decays of the type $\mathrm{J}^{\pi} \beta_{\rightarrow}^{+} \mathrm{J}^{\pi} \mathrm{J}^{\prime} \pi^{-}$, the $\beta-\gamma$ correlation measured with a detector that is sensitive to $\gamma^{-}$s with a specific circular polarization has the form ${ }^{2}$

$$
\omega\left(\omega_{e}, \theta\right)=1+\mathcal{E} / c A \cos \theta
$$

where

$$
\begin{aligned}
& A=\frac{1}{\sqrt{2}} \frac{1}{1+Y^{2}} \frac{2}{\sqrt{J(J+1)}}+4 Y \\
& \frac{\mathrm{F}_{1}\left(\lambda, \lambda, \mathrm{J}^{\prime}, \mathrm{J}\right)+2 \delta \mathrm{F}_{1}\left(\lambda, \lambda+1, \mathrm{~J}^{\prime}, \mathrm{J}\right)+\delta^{2} \mathrm{~F}_{1}\left(\lambda+1, \lambda+1, \mathrm{~J}^{\prime} \mathrm{J}^{\prime}\right)}{1+\delta^{2}} \\
& \theta=\text { the angle between the } \beta \text { and } \gamma \\
& \bar{E}=+1 \text { for right circular polarization and }-1 \text { for left circular } \\
& \text { polarization } \\
& \delta 2=\text { mixing ratio for the } \gamma \text { transition } \\
& \mathrm{F}=\text { angular correlation } \mathrm{F} \text { coefficients }
\end{aligned}
$$


The situation in the $\beta^{+}$decay of $24 \mathrm{Al}$ is similar to the one just described. The ${ }^{24} \mathrm{Al}$ ground state has $\mathrm{J}^{\pi}=4^{+}, \mathrm{T}=1$. The $\beta^{+}$. decays of ${ }^{24} \mathrm{Al}$ feed four different $\mathrm{J}^{\tilde{\pi}}=4^{+} \mathrm{T}=0$ levels in $24 \mathrm{Mg}$. This allows. us to measure four Fermi matrix elements instead of just one. In this way we hope to "map out" the anti-analog strength distribution as a function of excitation energy.

Our experimental arrangement to measure A consists of a plastic scintillator surface barrier telescope for detecting the $\beta^{+}$s and a polarimeter and $\mathrm{NaI}$ detector for detecting the $\gamma^{-}$s of a particular circular polarization. The plastic scintillator is a cylinder 5" in diameter and 2" deep; the surface barrier detector is an Ortec model TB-18-300-700 with an active area of $300 \mathrm{~mm}^{2}$ and a sensitive depth of 700 microns. The $\mathrm{NaI}$ and polarimeter are described in Ref. 3. The detectors are positioned so that the angle $\theta$ is $180^{\circ}$. The $24 \mathrm{Al}$ is made by the reaction $24 \mathrm{Mg}(\mathrm{p}, \mathrm{n}){ }^{24} \mathrm{Al}$ induced by $18 \mathrm{MeV}$ protons. The ${ }^{24} \mathrm{Al}$ is made in the $0^{\circ}$ beam line then shuttled to cave 2 by the rabbit (the rabbit is described in Section 9.3 of this report). The $24 \mathrm{Mg}(\mathrm{p}, \mathrm{n}){ }^{24} \mathrm{Al}$ makes both $24 \mathrm{Al}$ in the ground state and the first excited state. The ground state has a half life of $2.07 \mathrm{sec}$ and the first excited state has a half-1ife of $130 \mathrm{~ms}$. Therefore, the transit time for the rabbit is set at $400 \mathrm{~ms}$ so that essentially all of the excited state has decayed away before counting is begun in cave 2 .

The polarimeter will then be flipped prior to each counting period of the rabbit so that the polarimeter will respond alternately to right circular polarization $(+)$ and left circular polarizations ( - ). Then using equation (2)

$$
A \propto \frac{N_{+}-N_{-}}{N_{+}+N_{-}}
$$

where

$$
\begin{aligned}
& \mathrm{N}_{+}=\# \text { of coincidences with the polarimeter in the }+ \text { mode } \\
& \mathrm{N}_{-}=\# \text { of coincidences with the polarimeter in the - mode }
\end{aligned}
$$

We have measured betas in coincidence with gammas transmitted through the polarimcter and achleved a time resolution of about $3 \mathrm{~ns}$ for these coincidences. However our counting rate (limited by pileup of the slow linear signals from the scintillator) was too low for a practical measurement of $A$. At present we are improving our experimental arrangement by installing a fast-gated integrating ADC to process the $\beta^{+}$energy signal. This will greatly increase our counting rate and permit us to measure $A$ in approximately one week of running time.

\section{$\underline{\text { References }}$}

1. A.B. McDonald and E.G. Adelberger, Phys. Rev. Lett. 40, 1692 (1978).

2. S. Raman et al.; Atomic and Nuclear Data Tables, 16, 451 (1975).

3. Nuclear Physics Laboratory Annual Report, University of Washington p. 13 . 
3. Elastic and Inelastic Polarized Proton Scattering Via Isobaric Analog Resonances in $207 \mathrm{Bi}$ and $209 \mathrm{Bi}$

N.L. Back, H.C. Bhang, J.G. Cramer, D. Leach, T.A. Trainor, and

R. Von Lintig

We have extended our measurements of the elastic and inelastic scattering of polarized protons from $206 \mathrm{~Pb}$ and ${ }^{208} \mathrm{~Pb} .1$ Cross-section and analyzing power excitation functions have now been measured for both targets between 14.25 and $18.00 \mathrm{MeV}_{3}$ i.e., in the region of the single-particle isobaric analog resonances (IAR) in ${ }^{209} \mathrm{Bi}^{*}$. These resonances are observed in the elastic scattering and the scattering to the first $3^{-}$state in each target, and in the scattering to the first $2^{+}$state in $206 \mathrm{~Pb}$.

A number of changes have been made in the experimental techuique. The polarized ion source was operated in the fast-flip rather than the spin-filter mode, with the spin tlipped every 100 usec. The cncrgy eignals were rnuted into spin-up and spin-down bins by the computer. Thus, systematic effects resulting from the passage of time between spin-up and spin-down runs were eliminated. Also, the background in the spectra was reduced by placing permanent magnets

Fig. 3.3-1: Cross-section and analyzing-power excitation functions for $206 \mathrm{~Pb}\left(\overrightarrow{\mathrm{p}}, \mathrm{p}_{\mathrm{o}}\right)$, The curves are a fit using nine IARs and a parameterized background.
$\operatorname{Pb} 206(p, p) \quad$ The $t a=150$
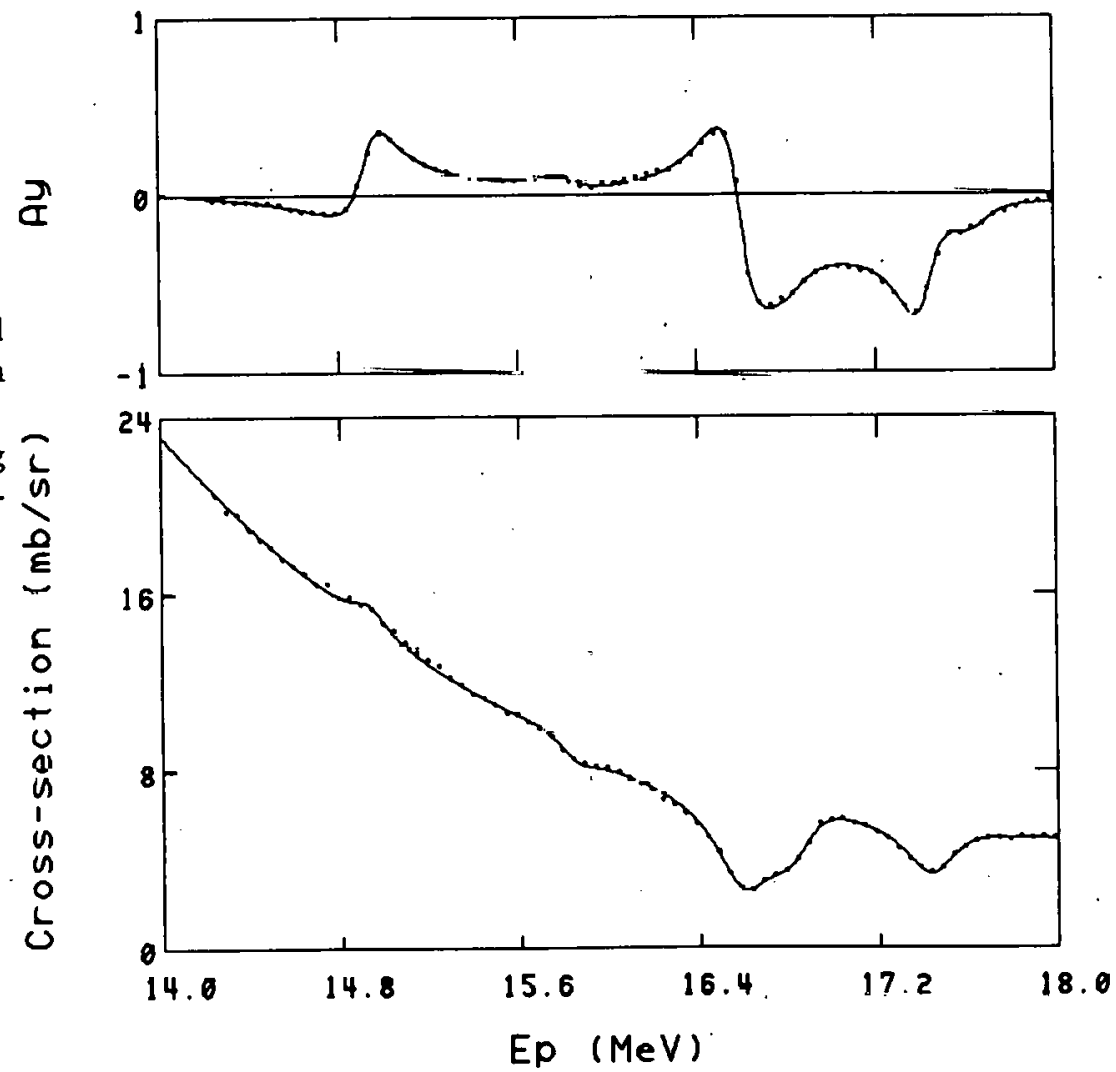
$\operatorname{Pb} 206\left(p, p^{\circ}\right) 2+(0.803 \mathrm{MeV})$ The $t a=150$

Fig. 3.3-2: Cross-section and analyzing-power excitation functions for $206 \mathrm{~Pb}\left(\overrightarrow{\mathrm{p}}, \mathrm{p}^{\prime}\right)$ to the $21^{+}(0.8033 \mathrm{MeV})$ state.
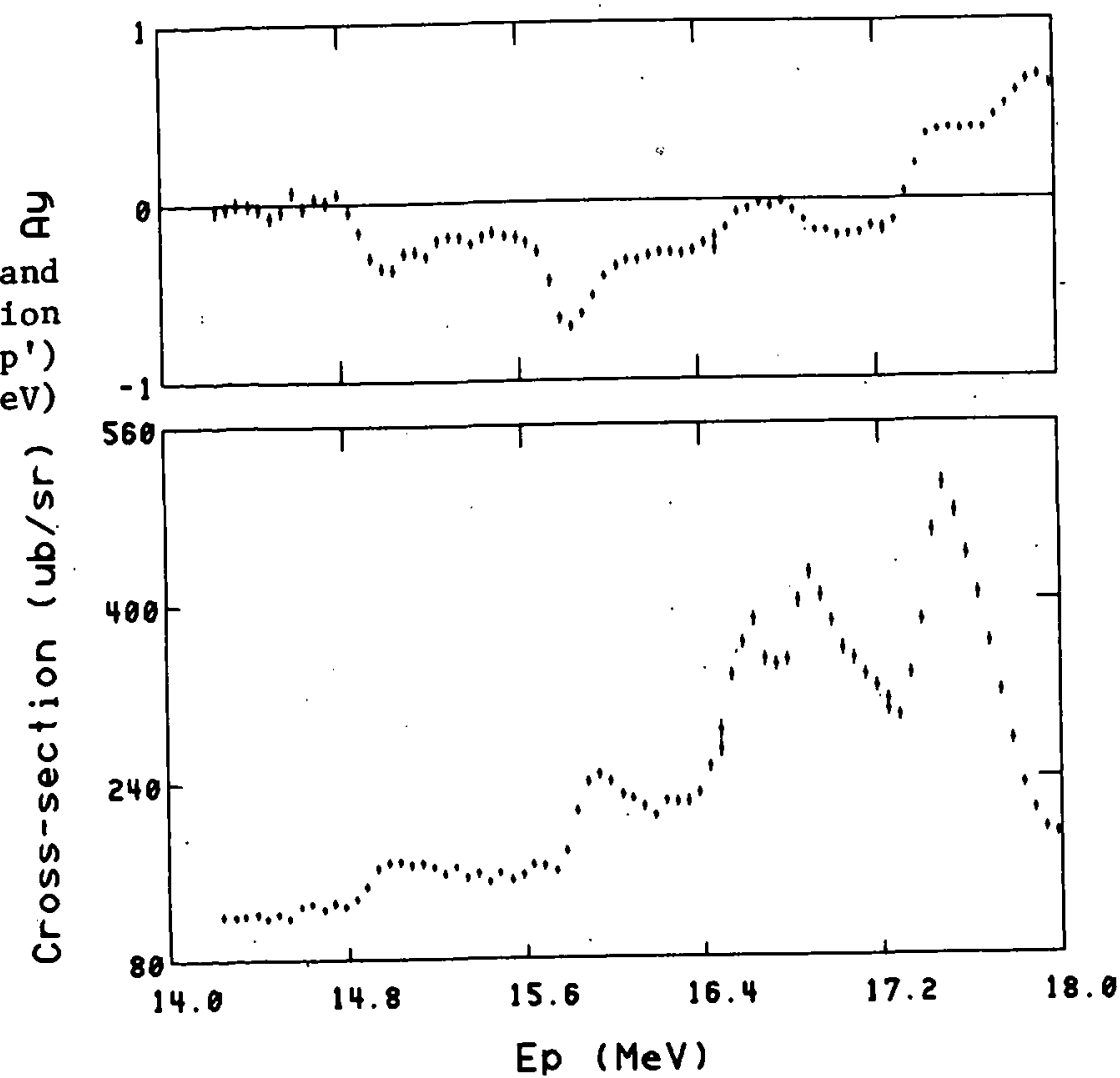

between the detectors and their. forward apertures, so that any electrons produced by the target were first collimated by the apertures. (located approximately halfway between the target and the detectors) and then deflected by the magnets.

In addition to the excitation functions, runs were taken at $4,6,8,10$, 12 , and $13.75 \mathrm{MeV}$. with unpolarized beam to aid in the normalization of the cross-section data. The data cannot simply be normalized to Rutherford scattering at $4 \mathrm{MeV}$; because of multiple scattering in the target at this energy, as much as $15 \%$ of the beam passing through the target hits the chamber walls rather than being collected by the Faraday cup. However: (a) the ratios of the solid angles of the detectors can be obtained from the $4 \mathrm{MeV}$ data; and (b) the indicated ratio to Rutherford for the monitor is constant (within $1 \%$ ) from 6 to $10 \mathrm{MeV}$, and so the $8 \mathrm{MeV}$ monitor data were used for the absolute normalization.

Angular distributions were also measured for each target at $13.75,15.50$, and $18.00 \mathrm{MeV}$. These will be used to find the best optical-model parameters for the analysis of the excitation-function data. 
$\operatorname{Pb} 206\left(p, p^{\circ}\right) \quad 3-(2.647 \mathrm{MeV}) \quad$ The $t a=156$

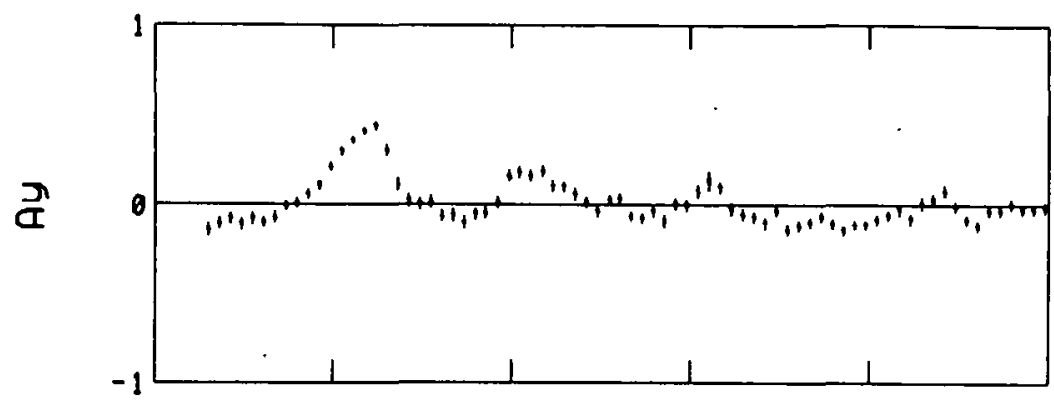

Fi.g, 3.3-3: Cross-section and analyzing-power excitacion functions for ${ }^{206} \mathrm{~Pb}\left(\overrightarrow{\mathrm{p}}, \mathrm{p}^{\prime}\right)$ to the $31^{-}(2.647 \mathrm{MeV})$ stale.

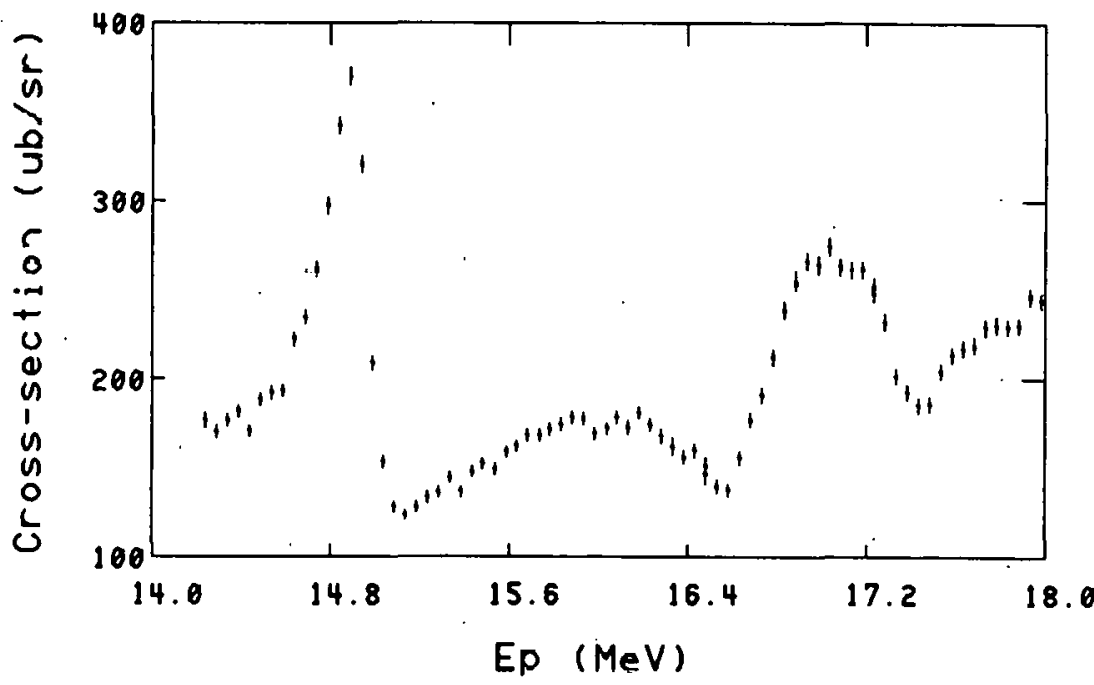

The data for $206 \mathrm{ph}$ at $150^{\circ}$ are shown in Figs. 3.3-1 through 3.3-3. The smooth curves shown in Fig. 3.3-1 represent a tit ro Llie data using 3 parameterized-background approach. In this approach, each background amplitude is expressed as a Taylor series with terms up to second order in $\left(E-E_{O}\right)$, where $E_{o}$ is the average of the highest and lowest energies being considered (except that the non-spin-fl1p amplitude also contains a term proportional to $\mathrm{E}^{-1}$ ). The background coefficients and resonance parameters are allowed to vary simultaneously to fit the data. This procedure is followed for each angle separately, and then the results are compared for consistency. It should be pointed out that the fits are not as good at other angles, especially at $165^{\circ}$; more detailed analysis is required before unambiguous spin assignments are made or final resonance parameters are extracted.

The results obtained so far for ${ }^{209} \mathrm{Bi} *$ are identical to those found by Baker et al. ${ }^{2}$ The resonances observed are the isobaric analogs of the low-lying single-neutron states in $209 \mathrm{~Pb}$, although the $j_{15 / 2}$ IAR is too weak to be seen.

The resonance structure in $20{ }^{\mathrm{Bi}}$ * is considerably more complicaled. Ilere there is considerable mixing between single-particle states built on a $206 \mathrm{~Pb}$ ground-state core and those built on a $2^{+}$core. As a result, the single-particle strength for each $(\ell, j)$ is split among several IARs. This mixing is also the reason for the appearance of IARs in the scattering to the $2^{+}$ state of $206 \mathrm{~Pb}$. 
Preliminary results for $207_{\mathrm{Bi}}$ * are listed in Table 3.3-1. The resonance energies (relative to the $g_{9 / 2}$ IAR) were determined by identifying the IARs with some of the states observed in the ${ }^{206} \mathrm{~Pb}(\mathrm{~d}, \mathrm{p})$ reaction. ${ }^{3}$ IARs corresponding to many of the weaker states have not yet been identified, but some of them will be required to improve the fit to the data. The column labeled "Previous Spin Assignment" contains the spin-parity assignments given in.Ref. 3 . Where the numbers are in parentheses, the assignments are uncertain.

When the analysis of the elastic scattering is completed, it will be possible : to determine the partial widths for decay of the IARs to the $2^{+}$and $3^{-}$ states. For this purpose, the "best" set of optical-model parameters will be used to generate the DWBA amplitudes, which interfere with the resonance amplitudes to produce the observed excitation functions.

\section{References}

1. Nuclear Physics Laboratory Annual Report, University of Washington (1979), p. 53.

2. Nuclear Physics Laboratory Annual Report; University of Washington (1974), p. 85.

3. Table of Isotopes, 7 th Edition, edited by C.M. Lederer and V.S. Shirley (John Wiley and Sons, New York, 1978). 
Table 3.3-1: Resonance Parameters for IARs in ${ }^{207}{ }_{B i *}$ (Pre1,iminary)

\begin{tabular}{|c|c|c|c|c|}
\hline$\underline{l_{1}}$ & $\begin{array}{c}\begin{array}{c}\text { Previous } \\
\text { Spin } \\
\text { Assignment }\end{array} \\
\end{array}$ & $\Gamma_{\mathrm{p}}(\mathrm{keV})$ & $\Gamma(\mathrm{keV})$ & $\mathrm{E}_{\mathrm{R}}\left(\mathrm{MeV}-\mathrm{c} \cdot \mathrm{m}_{\cdot}\right)$ \\
\hline$g_{9 / 2}$ & $g_{9 / 2}$ & 19.7 & 191 & 14.851 \\
\hline $\mathbf{i}_{11 / 2}$ & $\left(i_{11 / 2}\right)$ & 2.2 & 338 & 15.632 \\
\hline $\mathrm{d}_{5 / 2}$ & $d_{j / 2}$ & 5.0 & 229 & 15.7 .57 \\
\hline$d_{5 / 2}$ & $\left(d_{5 / 2}\right)$ & 0.7 & 269 & 16.442 \\
\hline$d_{5 / 2}$ & $\mathrm{~d}_{5 / 2}$ & 32.5 & 269 & 16.510 \\
\hline $\mathrm{s}_{1 / 2}$ & $\mathbf{s}_{1 / 2}$ & 44.7 & 275 & 16.750 \\
\hline$g_{7 / 2}$ & $(87 / 2)$ & 15.6 & 288 & 17.304 \\
\hline$d_{3 / 2}$ & $\left(d_{3 / 2}\right)$ & 43.2 & 279 & 17.342 \\
\hline $\mathrm{g} 7 / 2$ & $\left(\mathrm{~d}_{5 / 2}, \mathrm{~g}_{7 / 2}\right)$ & 8.3 & 288 & 17.551 \\
\hline
\end{tabular}

4. Low Lying Vibrational States in $6.4 . \mathrm{Zn}$

I.S, Blair, K.J. Davis, D.W. Storm, and T.A. Trainor

The ${ }^{64} \mathrm{Zn}$ nucleus exhibits $0^{+}-2^{+}-4^{+}$triplet of states near $1.91 \mathrm{MeV}$ excitation energy which is thought to be a set of "two-phonon" vibrational states built on the "one phonon" $2^{+}$state at $1.0 \mathrm{MeV}$. The $2^{+}$and $4^{+}$members of this triplet have been seen in a previous experiment using inelastically scattered alpha particles. 1 In that experiment the oscillations in the angular distributions of the $2^{+}$and $4^{+}$states were found to be out of phase with those predicted for a one-step excitation process. Such an angular distribution is qualitatively consistent with a cwo-step exeitation model. Our experiment is an attempt to resolve the relatively weak $0^{+}$peak, along with the $2^{+}$and $4^{+}$peaks, and make an angular distríburlon mea surement. 
In order to maximize the yield in this reaction we use alpha particles of $27 \mathrm{MeV}$, the highest energy available from our accelerator. The yield from the elastic peak is much greater than that, of the $0^{+}$inelastic peak $\left(\sigma_{\text {elastic }} / \sigma_{\text {inelastic }} \sim 10^{5}\right.$ at $\left.\theta=16^{\circ}\right)$. Consequently, special care must be taken to avoid slit scattering which can produce a long tail on the elastic peak.

Elastic peaks from contaminant $16_{0}$ and ${ }^{12} \mathrm{C}$ are also a problem, since they are in the vicinity of the two-phonon triplet for angles between $25^{\circ}$ and $45^{\circ}$. In our first run we found the contaminant peaks to be about two orders of magnitude larger than the $0^{+}$peak at $50^{\circ}$. Kinematic broadening is also fairly serious $\left(\sim 40 \mathrm{keV} / \mathrm{deg}\right.$ at $\theta=50^{\circ}$ ) so it is necessary to use small beam defining and detector apertures.

In our first run we were able to get an overall resolution of about $55 \mathrm{keV}$. This was sufficient for us to resolve the $0^{+}$and $2^{+}$peaks (at $50^{\circ}$ ) which are separated by $111 \mathrm{keV}$. However, the counting rate in that experiment was quite low ( 300 counts in the $0^{+}$peak after 8 hours of counting with a $200 \mathrm{nA}$ beam on a $100 \mathrm{\mu g} / \mathrm{cm}^{2} 64 \mathrm{Zn}$ target). Our efforts since then have been directed towards increasing the counting rate. We feel that a factor of 10 increase can be achieved by increasing the detector aperture width, increasing the target thickness and increasing the beam current. The last can be achieved only by enhancing target cooling since the target has a low melting point. This can be accomplished by making a smaller hole in the target frame in order to improve heat conduction.

Our future work will include, in addition to a measurement of angular distributions, an investigation of the excitation functions to check for the possible presence of energy fluctuations.

Granted that such fluctuations are absent, we will relate the measured angular distributions to coupled channel calculations which allow for direct two-step as well as one-step collective excitations, using either code JUPITOR ${ }^{2}$ or ECIS. 3

\section{References}

1. N. A1pert et a1., Phys. Rev. C2, 974 (1970).

2. T. Tamura, Rev. Mod. Phys. 37, 679 (1965).

3. J. Raynal, Saclay Report No: $\overline{\mathrm{DPh}}-\mathrm{T} / 71-48,1971$ (unpublished). 


\section{Depolarization in the Inelastic Scattering of Protons from Copper}

H. Bhang, S.K. Lamoreaux, S. Laubach, ${ }^{+}$I. Hal pern, T.A. Trainor, and U.G. Weitkamp

We are continuing an experimental survey of the depolarization parameter in inelastic scattering of protons from continuum states in medium weight nuclei such as copper. This survey is intended to provide additional information on the mechanism of inelastic scattering. There are many studies of spin observables such as the analyzing power or the depolarization to discrete states in nuclei, especially light nuclei, and there are now several recent measurements of analyzing power in inelastic scattering to the continuum, but the measurements reported here constitute the first measurements of the depolarization in inelastic scattering to the continum that we are aware of.

The depolarization parameter $\mathrm{D}$ ( or $\mathrm{K}_{\mathrm{y}} \mathrm{y}^{-}$) is approximately the ratin of incident beam polarization to outgoing particle polarization. More precisely, it is given by

$$
\mathrm{D}=\left[\mathrm{P}_{\mathrm{y}}-\left(1+\mathrm{P}_{\mathrm{y}} \mathrm{A}_{\mathrm{y}}\right)-\mathrm{P}^{-}\right] / \mathrm{p}_{\mathrm{y}},
$$

where $p_{y}$ and $p_{y}$ - are the polarizations of the incident and emitted protons respectively, $A_{y}$ j.s the analyzing power, and $\mathrm{Py}^{\prime}$ is the polarization resulting from an unpolarized incident beam. In inelastic scattering from medium weight nuclei, both $A_{y}$ and $P^{-}$are essentially zero. D is related to the spin flip probability S:

$$
S=(1-D) / 2 \text {. }
$$

The depolarization parameter has a value of unity when no change in the polarization occurs, and a value of -1 when the spins of all the protons are flipped in the scattering process. If the protons are completely depolarized, the depolarization parameter is zero.

Experimental Technique

To measure the depolarization, we need a beam of polarized protons, which our tandem produces in intensities up to $150 \mathrm{nA}$ at $18 \mathrm{MeV}$. The beam polarization is measured with a polarimeter described in sec. 9.11 of this report.

To measure the polarization of the protons leaving the nucleus, we use a helium polarimeter, which has been described previously. ${ }^{2}$ The polarimeter has been designed to maximize the counting rate and minimize the background. To maximize the counting rate we make the target thick, 14 atmospheres of helium, and the angular spread large. This leads to rather poor resolution: from 1 to $1.7 \mathrm{MeV}$ FWHM. To minimize background, we use a counter telescope consisting of two proportional counters and a silicon detector. The entire polarimeter is made of iron to reduce the flux of neutrons from the target striking the detectors. There is a door in front of the polarimeter that can be closed to exclude charged particles for measurement of neutron-induced backgrounds. 
The helium polarimeter works quite well. The peak-to-background ratio observed when measuring protons entering the polarimeter after scattering from gold is about 50:1 so that we need not worry about energy degraded protons interfering with the measurement. The energy calibration of pulses from the silicon detectors in the polarimeter is determined with protons scattered from carbon, leaving the carbon in the ground, 4.439 or $9.632 \mathrm{MeV}$ states.

The analyzing power calibration of this polarimeter is described in Sec. 10.8 of this report. The computer programs used to process data from the polarimeter and calculate values of the depolarization parameter as a function of excitation energy are described in Sec. 10.9 of this report.

Data

Figure 3.5-1 shows some of the data we have taken. At the low excitation energy end of the spectrum, the depolarization parameter is essentially unity, indicating that spin flip is not a significant factor in exciting the majority of states here. Note that the resolution of our polarimeter completely wipes out any structure; we average over about $1.7 \mathrm{Mev}$ at low excitation energies. At the high excitation energy end, the depolarization has a value near zero, indicating that the outcoming protons are essentially unpolarized. In between the depolarization drops fairly smoothly with increasing excitation energy. The data show essentially the same shape at all angles. This is surprising, since the inelastic cross sections show somewhat different shapes at $45^{\circ}$ and $135^{\circ}$.

Fig. 3.5-1: Depolarization in proton scattering from $63 \mathrm{Cu}$

Interpretation

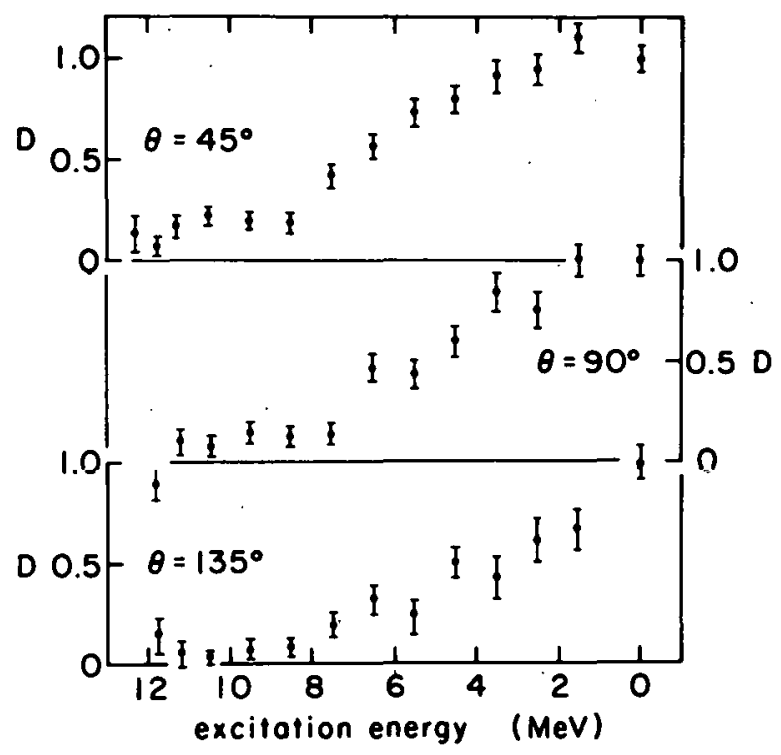

For reference, the proton spectrum resulting from bombarding ${ }^{63} \mathrm{Cu}$ with 18 $\mathrm{MeV}$ protons is shown in Fig. 3.5-2, plotted as a function of excitation energy. It is convenient to divide the excitation energy into three regions (depending on the reaction mechanism involved in the inelastic scattering): the direct reaction region from 0 to about $6 \mathrm{MeV}$ excitation, the evaporation region above $12 \mathrm{MeV}$, and the pre-equilibrium region from about 6 to $12 \mathrm{MeV}$. The depolarization parameter is expected to show rather different behavior in these three regione. 
Fig. 3.5-2: Cross section for proton scattering from ${ }^{63} \mathrm{Cu}$

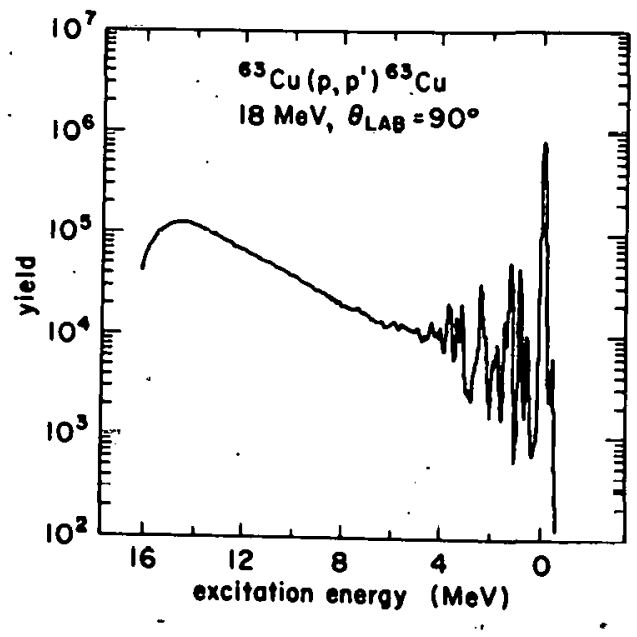

Direct Reaction Region. In the region of lowest excitation, the yield is dominated by scattering to discrete states. As mentioned above, it is clear from the data that spin flip is not an important process in exciting these states. This is to be expected since in those cases where spin flip in inelastic scattering to discrete states has been measured, it is generally quite small, i.e., D is approximately unity. ${ }^{3}$

The Evaporation Region. At excitation energies from 12 to $16 \mathrm{MeV}$, the highest energies at which a proton can still escape from the nucleus, the spectrum is a continuum as a function of energy and the cross section is well1 described by statistical theory. ${ }^{4}$

One can make a simple minded prediction for the depolarization parameter in the evaporation region. If $z_{e}$ is the number of protons in the target nucleus which can evaporate, and if one assumes that the incident proton is not depolarized, the depolarization parameter should be approximately $1 /\left(z_{e}+1\right)$, since half of the protons which can evaporate have their spins pointing in the direction opposite to the incident proton. Our data give a hint that the depolarization parameter doesn't quite go to zero at high excitation energies.

The Pre-equilibrium Region. The reaction mechanlsw in the pre-equilibrum region between 5 and $12 \mathrm{MeV}$ is not well understood. There are theoretical calculations predicting the cross section, but no predictions of the depolarization parameter. 
In the pre-equilibrium region, the depolarization decreases with increasing excitation energy. This is in part due to the increasing admixture of evaporated protons (presumably unpolarized) with directly scattered protons (presumably polarized). One can use statistical theory to "correct" $D$ for the effects of these evaporated protons. A preliminary calculation shows that $D$ so corrected still falls toward zero with increasing excitation energy. The "corrected" D, for example, is about 0.2 higher at $6 \mathrm{MeV}$ excitation than the uncorrected value.

There are several possible explanations for the observed decrease in $D$ with excitation energy. One is that the depolarization results from spin flip in the nucleon-nucleon interaction. It may be possible to relate available data to the scattering process involved in the nucleus and calculate $D$.

A second explanation is based on conventional theories of pre-equilibrium proton emission. 6 It is hypothesized that an incident proton gives up energy to the nucleons in the nucleus by exciting particle-hole states. The more energy the proton loses, the larger the number of states which can be excited. At some point in the process, states, will be excited in which one of the protons in the nucleus is above the separation energy, so that the nucleus can decay by proton emission. Even if we assume that the incident proton experiences no spin flip, we can understand the depolarization falling off uniformly with increasing excitation energy by realizing that any proton leaving the nucleus, other than the incident proton, will have its spin in an arbitrary direction. Thus the outgoing protons will be less highly polarized than would have been the case if o.ly the incident proton had left the nucleus.

In the near future, we expect to extend the measurements to nuclei with somewhat higher and lower $Z$. We also hope to be able to develop a quantitative explanation for the features of these data.

\section{$\underline{\text { References }}$}

+ Visitor from the University of Darmstadt, Germany.

1. Sec. 3.7 of this report.

2. Nuclear Physics Laboratory Annual Report, University of Washington, 1979, p. 157 .

3. J.M. Moss, W.D. Cornelius and D.R. Brown, Phys. Lett. 71B, 87 (1977).

4. A. Sprinzak, A.J. Kennedy, J.C. Pacer, J. Wiley, and N.T. Porile, Nuc1. Phys. A203, 280 (1973).

5. T.C. Griffith, D.C. Imrie, G.J. Lush, and A.J. Metheringham, Phys. Rev. Lett 10, 444 (1963). br 6. E. Gadioli and E. Gadioli Erba, Nucl. Inst. and Meth. 146, 265 (1977). 


\section{Analyzing Power in the Continuum Portions of Particle Spectra}

H.C. Bhang, I. Halpern, and T.A. Trainor

In earlier Annual Reports ${ }^{1}$ we have described the method and the results of our studies of analyzing powers of $18 \mathrm{MeV}$ polarized protions on various targets. Very briefly, our findings for the $\left(p, p^{-}\right)$reaction to residual excitation energies between $5-10 \mathrm{MeV}$ is that the mean analyzing power in this interval is negative between $30^{\circ}-90^{\circ}$. It has a maximum in the middle of this angular interval of about 3\%. At angles in the backward hemisphere, the analyzing power is smaller and positive. These patterns apply to each of the nuclei we have studied in detail, ${ }^{63} \mathrm{Cu},{ }^{64} \mathrm{Zn}$, and ${ }^{103} \mathrm{Rh}$.

The fact that the analyzlug power does not fluctuate rapidily with angle or residual energy must be due to the averaging over spins and parities of the many final states that are reached. In any event, the uniformity of results with energy, angle and target nucleus suggests that these results should prove to be understandable in simple terms, and therefore that they would hopefully shed some light on the mechanism of the reactions being studied.

During the past year we have worked on the understanding of the observed analyzing powers. Recently there have appeared some results ${ }^{2}$ from 0 saka on proton analyzing powers to the continuum at an incident energy ( $65 \mathrm{MeV}$ ) higher than ours. To our knowledge, these data and ours are the only systematic data on this subject and we have examined both, in our efforts to understand the origin of the analyzing powers. The higher energy data have the oame otability with respect to target, and simplicity of pattern as ours do. The patterns however are not exactly the same.

We are currently in the midst of our studies of these patterns. The general program is this: Cross sections and analyzing powers are calculated with the DWBA code HEIMY. ${ }^{3}$ This code usos a collective form factur for ( $\ddot{p}, \mathrm{p}^{\prime \prime}$ ) and includes spin-orbit terms in their full Thomas form. The analyzing power at any excitation energy is an incoherent sum over momentum transfers $\mathrm{L}$.

$$
A_{y}(\theta)=\left(\frac{1}{\sigma_{0}}\right) \sum_{L} \beta_{T}^{2} \sigma_{L}(\theta) A_{L}(\theta)
$$

where

$$
\sigma_{0}=\sum_{L} \beta_{L}^{2} \sigma_{L}(\theta)
$$

Values of $\sigma_{L}(\theta)$ and $A_{L}(\theta)$ are computed using the DNBA code and then $A_{y}(\theta)$ values are computed under various assumptions about the $L$ dependence of the $\beta_{L}{ }^{2}$. 
Figure 3.6-1:

(a): Analyzing power for two strength distributions, $\left(\beta_{L}{ }^{2}\right)$, of the reaction $58 \mathrm{Ni}\left(\mathrm{p}, \mathrm{p}^{\prime}\right), \mathrm{Q}=22 \mathrm{MeV}$. Solid line, $B_{L}=$ constant. Dotted line, $\beta_{L}{ }^{2}(2 L+1)$ for $\mathrm{L}=0 \sim 6$.

(b): Solid line includes even L's only and dotted line inçludes odd L's only. Constant $\beta_{\mathrm{L}}{ }^{2}$ was used for both.

(c): Q-value depentence of $A_{y}$; solid line for $Q=14 \mathrm{MeV}$, fine dotted $\mathrm{Q}=22 \mathrm{MeV}$ and dot-dash line $\mathrm{Q}=$ $30 \mathrm{MeV}$.
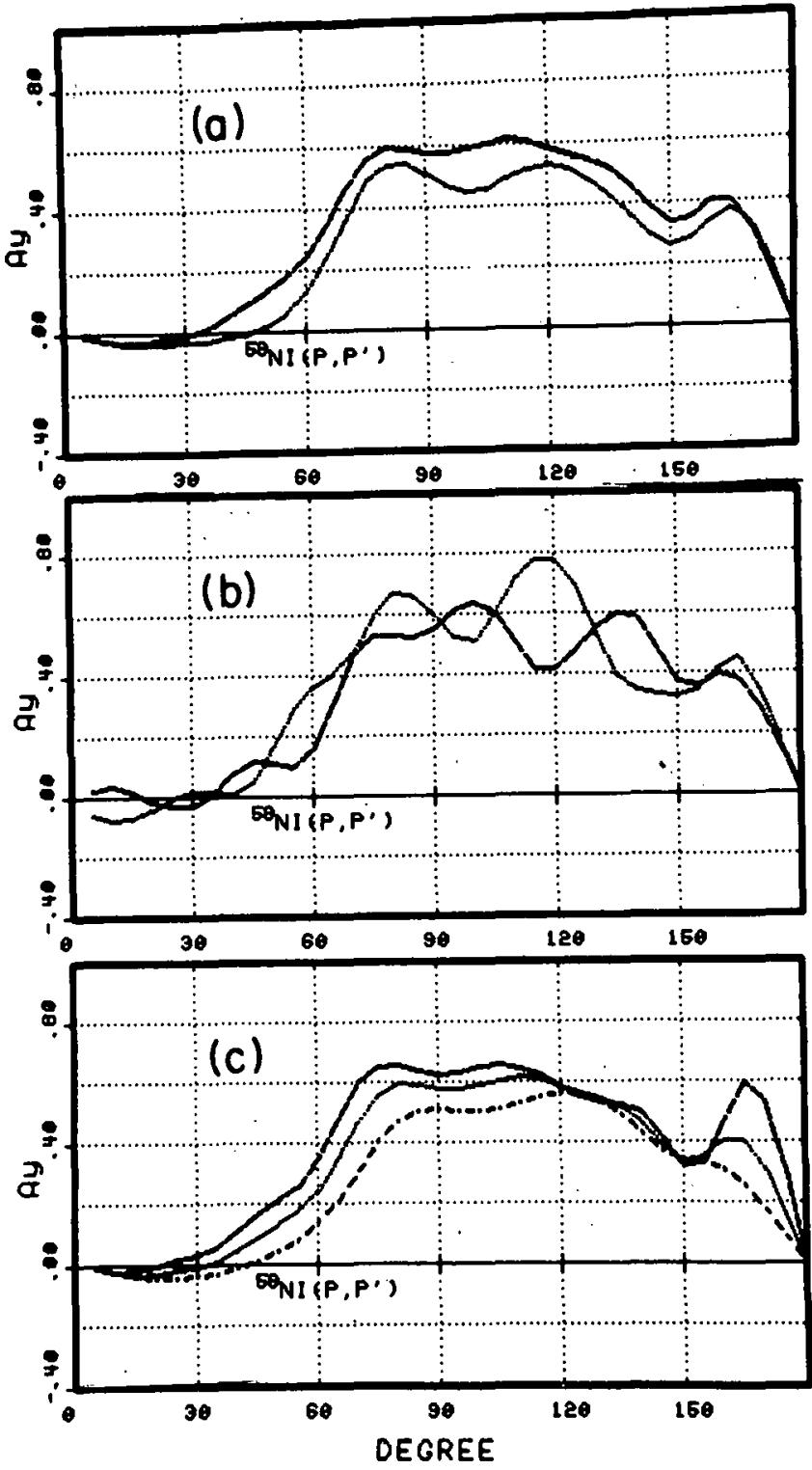

We should say at the outset that when reasonable values are used for various parameters in the calculation, the results reproduce the qualitative features of the experimental observations. In order to determine which physical parameters are critical for agreement with observations, we are engaged in exercises in which we change various parameters to see how they affect the computed analyzing powers. At the same time we are considering some quasi-classical models and hopefully we will include in these conceptually simple models all of the physical elements which the DWBA exercises show are required to reproduce the observed analyzing power patterns. 
In Fig. 3.6-1 we have illustratcd some of the results of these exercises which were carried out with parameters to match the Osaka data on Nickel. We use the Osaka data rather than our own for first comparisons because they cover a larger range of excitation energy and a bigger spread in $L$ values. The calculated $A_{y}(\theta)$ are qualitatively similar to the observed ones. Figure 3.6-la shows results where the $\beta_{\mathrm{L}}{ }^{2}$. were all assigned the same value (solid line) and where they were weighted by $2 L+1$ (dotted line). The point to be made is that the actual $\beta_{L}{ }^{2}$ distribution does not matter much as long as it is smooth and includes enough $\mathrm{L}^{-} \mathrm{s}$. This is shown in another way in Fig. $3.6-1 \mathrm{~b}$ where only even L's were used in one calculation (solid line) and only odd in another (dotted line): Again the general patterns are similar to each other and to those in Fig. 3.6-la. One can clearly see in Fig. 3.6-1b, however, some not fully damped oscillations which are connected to the parity of the L transfer and which romind one of analyzing power curves for final states of definite spin and parity.

Figurc 3.6-le shnws the calculated analyzing power as a function of $Q$, the proton energy lost in the scattering. Aydin the pattern is falrly stable with the analyzing power falling off slowly with increasing energy loss. The measured analyzing powers actually fall off more sharply with energy loss than the calculated ones. Sone of this may be due to contributions of two-step processes which are neglected in our calculation.

It must be pointed out that although the calculated patterns of analyzing power resemble those observed, there is a discrepancy as to magnitude. The calculated analyzing powers at backward angles are about three times larger than the measured ones. We thought at first that this discrepancy might be due to our failure to include multi-step scattering in the calculations and to our use of a collective form. factor for the scattering. We have recently learned that T. Tamura ${ }^{4}$ has also been looking at this problem and has included two-step scatterings. Tamura and his co-workers find that the inclusion of two-steps has little effect on $A_{y}$. They also find that the use of a microscopic form factor does reduce the calculated $A_{y}{ }^{\prime} s$ but not as much as is required by the data. Thus the discrepancy between theory and experiment in magnitudes of these back angle analyzing powers remains not fully accounted for.

Since the analyzing powers are rather flat with angle at these back angles, and since the angular distribution in $\left(p, p^{-}\right)$falls rather exponentially, i.e., $\sigma(\theta) \simeq \sigma_{0} \exp \left(-\theta / \theta_{0}\right)$ where $\theta_{0}=25^{\circ}$ for the Osaka data, one can express the back angle analyzing powers in terms of an angular displacement, $\Delta \theta=2 \theta_{0} A_{y}$, between the left and right scattering distributions. An analyzing power of 0.20 , for example, corresponds to an angular shift of $10^{\circ}$ between left and right curves. In the semi-classical models fur the analyzing power, it is often easiest to discuss the observation in terms of such an angle shift.

These considerations call attention to the point that a plot of $A_{y}$ vs. $\theta$ displays analyzing powers in regions of very small cross-section equally with those in regions of large cross-section. From the puint of vicw of dealing with average analyzing powers, a more even-handed way to display analyzing power is to plot of vs. $\theta$. The calculation shown in Fig. $3.6-1 c$ is replotted in this 


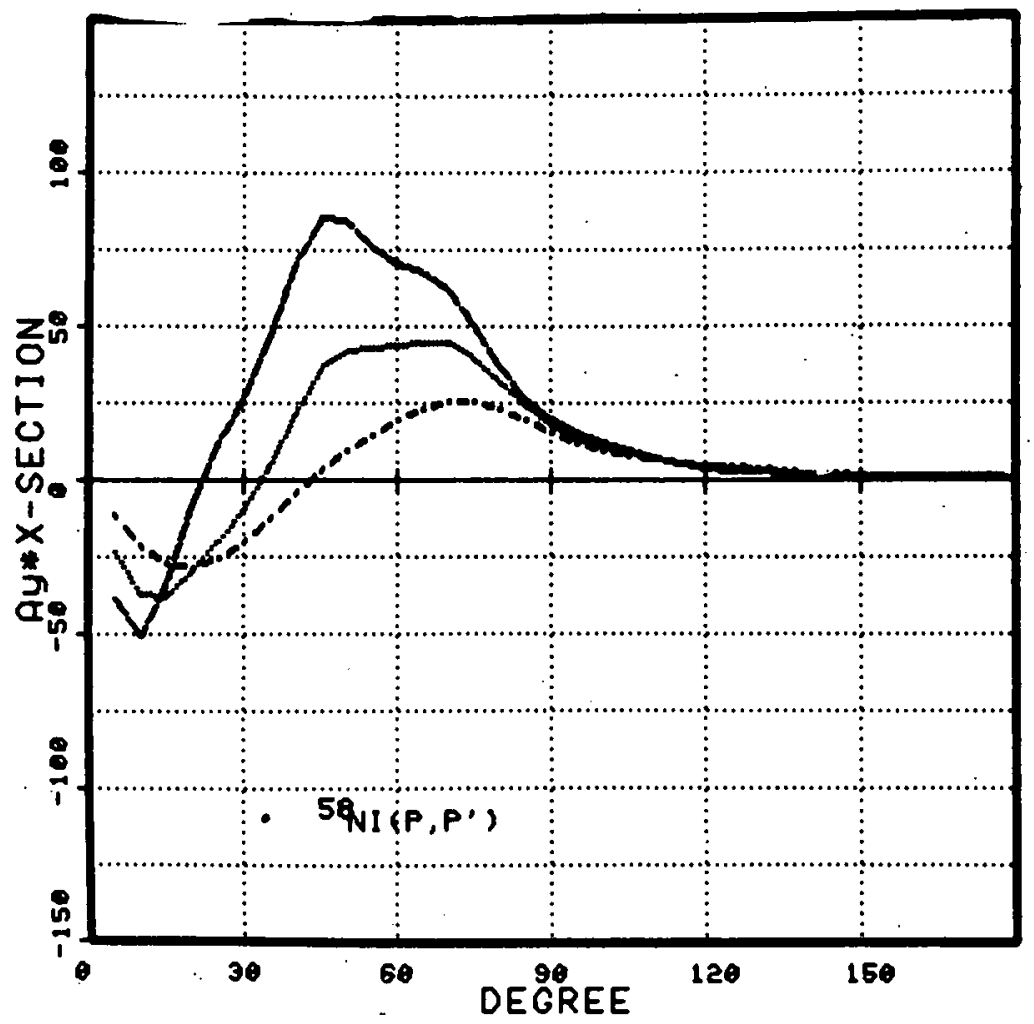

Fig. 3.6-2: Q-value dependence of $\sigma A_{y}$ solid line for $Q=14 \mathrm{MeV}$, fine dotted line $Q=22 \mathrm{MeV}$ and dot-dash line $Q=30 \mathrm{MeV}$.

Fig. 3.6-3: Analyzing powere calcul ated with DWBA for 5 different incoming energies from the top, $E_{p}=18 \mathrm{MeV}$ $(\mathrm{Q}=8 \mathrm{MeV}), 24 \mathrm{MeV}(\mathrm{Q}=10 \mathrm{MeV})$, $30 \mathrm{MeV} \quad(Q=12 \mathrm{MeV}), 40 \mathrm{MeV} \quad(Q=$ $17 \mathrm{MeV})$, and $50 \mathrm{MeV}(\mathrm{Q}=19 \mathrm{MeV})$. For 40 and $50 \mathrm{MeV}$ the optical potential adopted was that of Menet et al. 5 and Greenlees-Becchetti's global potential 6 was used for the 18, 24, and $30 \mathrm{MeV}$ calculations.

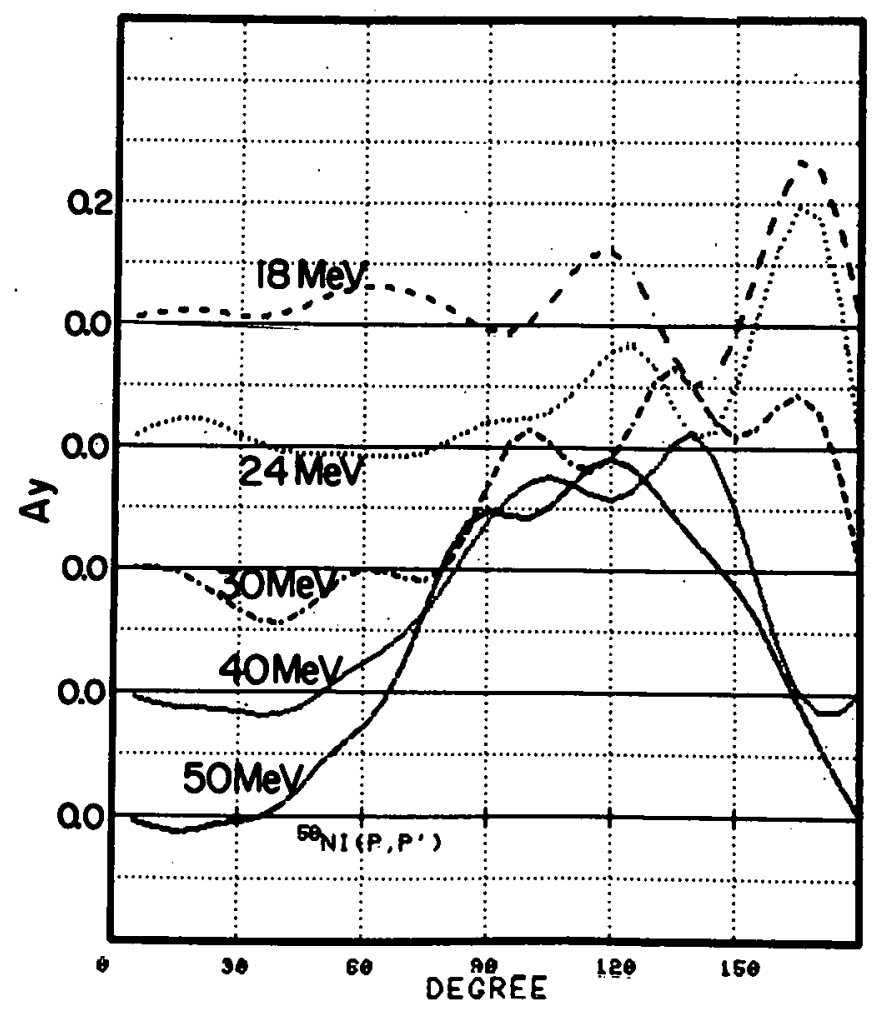


way in Fig. 3.6-2. It is seen that as the inelasticity of the scattering increases, the angle at which $\sigma \mathrm{A}$ goes from minus to plus moves to larger angle and that the relative amount of positive analyzing power falls off. These features can be reproduced by some very simple classical models for the scattering, but we have yet to establish that these models properly correspond to the physical content of the DWBA calculations which led to Fig. 3.6-2.

It has not been as straightforward to deal with our own $18 \mathrm{MeV}$ data as with the Osaka data because with lower incident energy (and lower $\mathrm{L}^{-} \mathrm{s}$ ) the calculated patterns tend to oscillate more. This feature is illustrated in plots of $A_{y} v s$. $\theta$. for different incident energies and residual energies of Fig. 3.6-3. By the time one gets down to $18 \mathrm{MeV}$, the analyzing power is very oscillatory and does not resemble our observations, which look somewhat more like those calculated tor $25 \mathrm{MeV}$. In any event the $18 \mathrm{MeV}$ data differs from the $65 \mathrm{MeV}$ data in having a longer angular region of negative analyzing power at forward angles. The DWBA calculations reproduce this trend as one decreases the incident energy.

The fact that our $\left(p, p^{-}\right)$and $(p, \alpha)$ data show similar analyzing power patterns and that the Osaka workers see ( $p, d)$ patterns like their $\left(p, p^{\prime}\right)$ patterns suggests that entrance channel effects, rather than exit channel or interaction effects, may be controlling as regards analyzing power in this energy range. We are continuing our calculational exercises to study the relative importance of spin-orbit effects at the different stages of the reaction. Our overall goal is to see what is the smallest number of simple and reasonable assumptions that are required to give a decent account of analyzing powers to the continuum. We have been helped immeasurably in these efforts hy discussions with J.S. Blair, G.A. Miller and H. Sherif.

\section{$\underline{\text { References }}$}

1. Nuclear Physics Laboratory Annual Report, University of washington (1979), p. 58 .

2. H. Sakai et al., Contribution to the International Symposium on Continuum Spectra of Heavy Ion Reactions, San Antonio, Dec. 1979.

3. H. Sherif, Dissertation, University of Washington (1968).

4. T. Tamura et al., Annual Report of Theorctical Nuclear Physics Group, University of Texas, 1980.

5. J.J.H. Menet et a1., Phys. Rev. C4, 111.4 (1971).

6. F.D. Becchetti, Jr. and G.W. Greenlers, Phyo. Rev. 182, 119 U (1969).

7. Higher $\mathrm{T}=3 / 2$ States in ${ }^{29} \mathrm{P}$ and ${ }^{25} \mathrm{Al}$

E.G. Adelberger, C.D. Hoyle, and P.G. Ikossi

The reduced elastic proton widths of the ground state analogs of $T=3 / 2$ states in $4 \mathrm{n}+1 \mathrm{~T}_{Z}=-1 / 2$ nuclei follow a strikingly simple dependence on the mass number. 1 The information on the widths of excited $\mathrm{T}=3 / 2$ states is limited. 
Fig. 3.7-1: Reduced elastic proton widths of $\mathrm{T}=3 / 2$ states in $17 \mathrm{~F}$ as a function of excitation energies. Data taken from Ref. 2 .

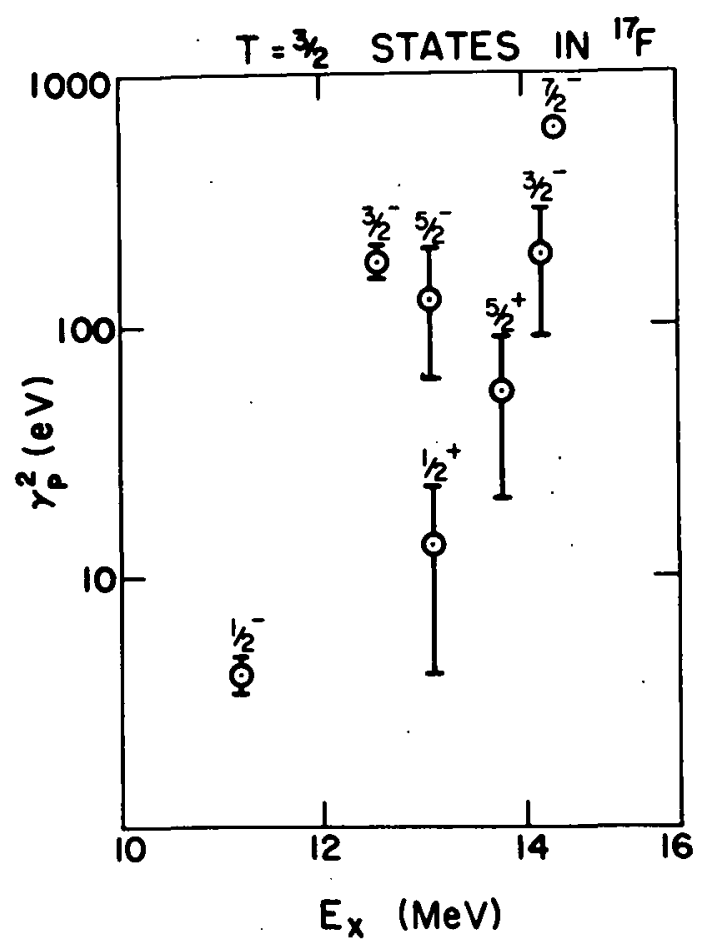

In the case of $17 \mathrm{~F}$ where several $\mathrm{T}=3 / 2$ excited states have been located the available evidence indicates that the reduced widths increase exponentially with the excitation energy of the states (Fig. 3.7-1).2,3 Whether this behavior is universal (perhaps indicating a correlation of isospin admixtures with the level density of $\mathrm{T}=1 / 2$ levels) or accidental needs to be tested in other nuclei. Another interesting question that may be answered by a systematic study of excited $\mathrm{T}=3 / 2$ states is the unexplained $\mathrm{A}=8$ periodicity observed in the reduced elastic proton widths. If this periodicity is due to the structure of the ground state of the target nuclei one may expect that on the average it will persist in the elastic proton widths of the excited $T=3 / 2$ states. Systematic studies of higher $\mathrm{T}=3 / 2$ states may thus provide useful information on the mechanism responsible for the isospin forbidden widths.

As an extension of our proton scattering experiments we have searched for excited $\mathrm{T}=3 / 2$ states in ${ }^{25} \mathrm{Al}$ and ${ }^{29} \mathrm{P}$. We have located resonances which are good candidates for the second excited $T=3 / 2$ states in both nuclei.

A. The Second Excited $\mathrm{T}=3 / 2$ state in ${ }^{25} \mathrm{Al}$

This state is the analog of the $\mathrm{J} \pi=1 / 2^{+} \mathrm{E}_{\mathrm{X}}=1.07 \mathrm{MeV}$ excited state of ${ }^{25} \mathrm{Ne}$. In ${ }^{25} \mathrm{Si}$ the corresponding state occurs at an excitation energy of 0.82 MeV. 4 Based on these excitation energies and the location of the lower $\mathrm{T}=3 / 2$ states in $25_{\mathrm{A} 1}$ we expect the $\mathrm{T}=3 / 2,1 / 2^{+}$state in $25 \mathrm{Al}$ to be located at $\sim 8.9$ $\mathrm{MeV}$. We have searched in $1.4 \mathrm{keV}$ steps using a $10 \mu \mathrm{g} / \mathrm{cm}^{2}$ thick target in the incident proton range of 6.8 to $7.3 \mathrm{MeV}\left(E_{\mathrm{x}}=8.8-9.3 \mathrm{MeV}\right)$. The only narrow resonance consistent with JII $-1 / 2^{+}$observed occurs at $E_{p}=6.857 \mathrm{MeV}$ (see Fig. 3.7-2) which corresponds to $E_{x}=(8.853 \pm 0.005) \mathrm{MeV}$. 


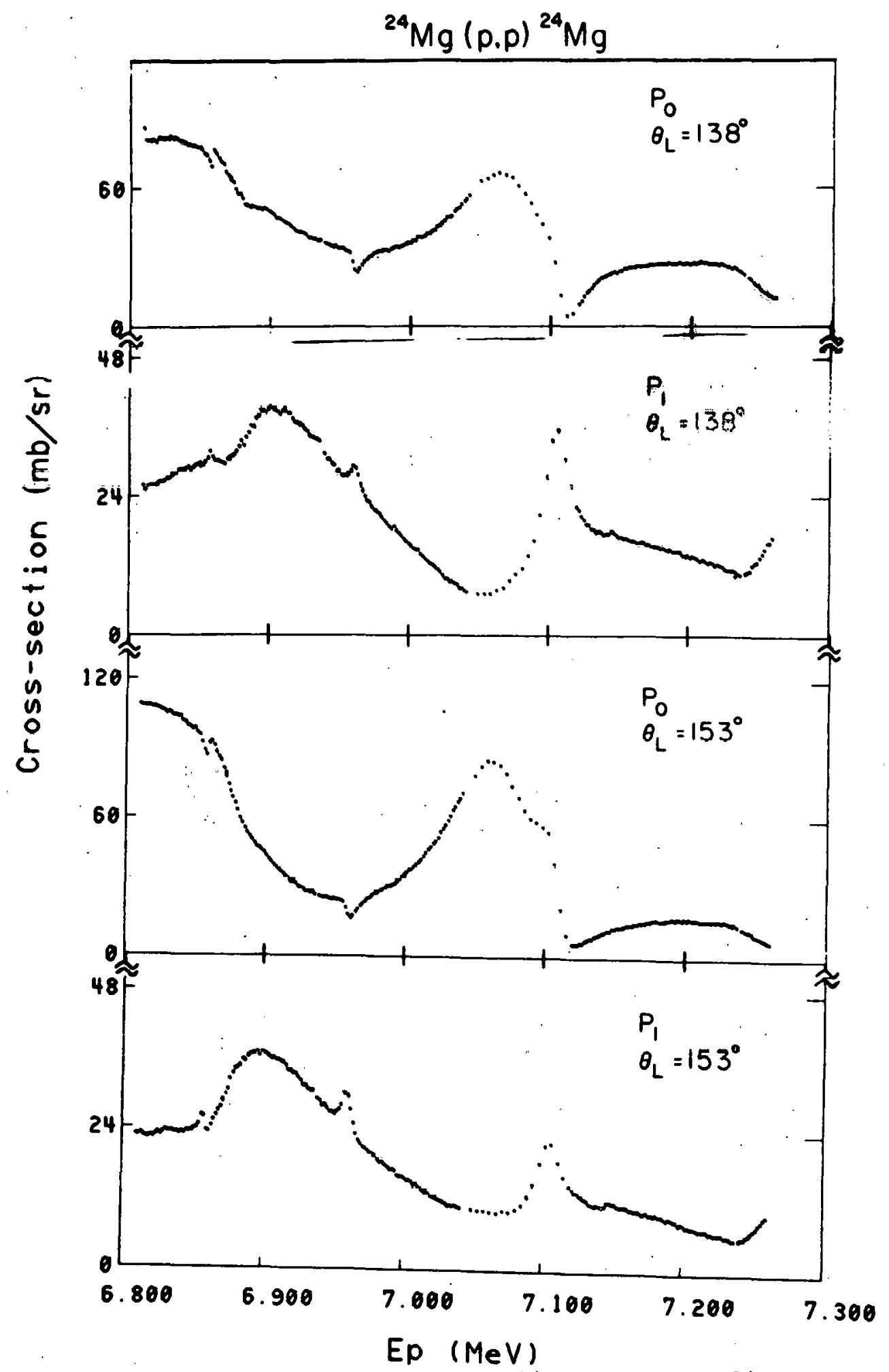

Fig. 3.7-2: Excitation functions for the $24 \mathrm{Mg}\left(\mathrm{p}, \mathrm{p}_{\mathrm{o}}\right)$ and ${ }^{24} \mathrm{Mg}\left(\mathrm{p}, \mathrm{p}_{1}\right)$ reaction in the region of the second excited $\mathrm{T}=3 / 2$ state in ${ }^{25} \mathrm{Al}$. 


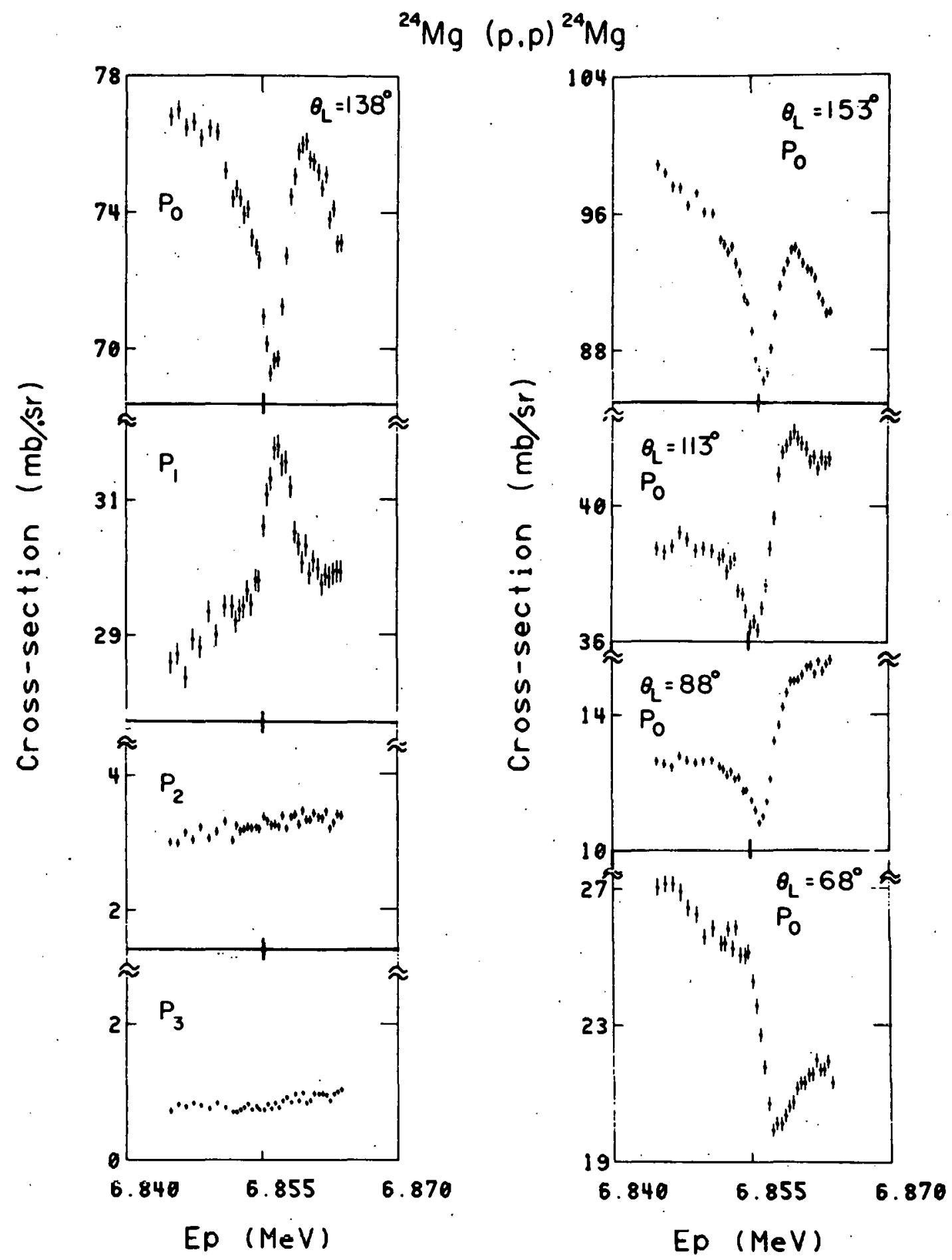

Fig. 3.7-3: Fine step excitation function over the $8.853 \mathrm{MeV}$ state. Note that the presence of a resonance effect at all angles indicating $\mathrm{J}^{\pi}=1 / 2^{+}$. 


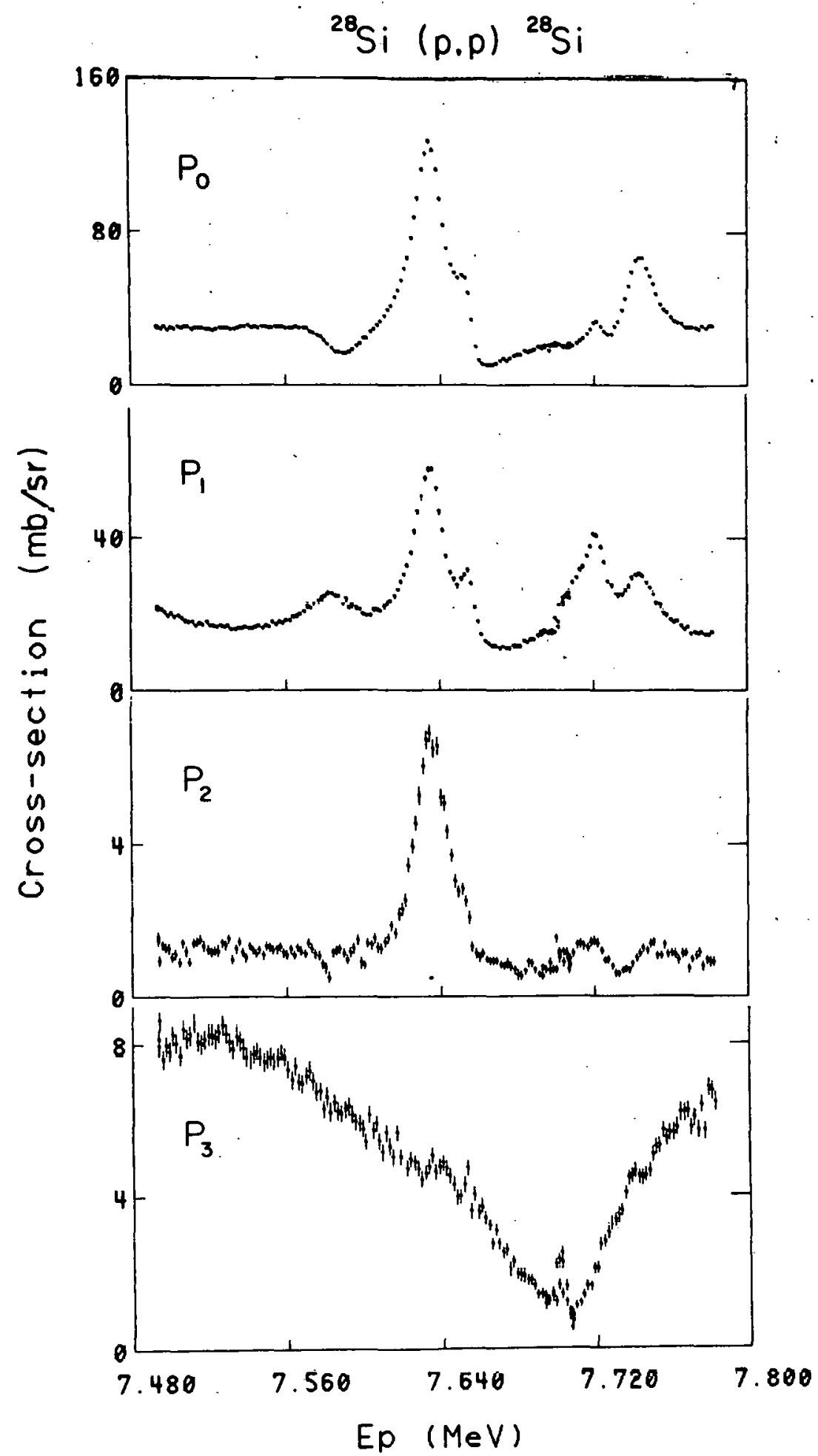

Fig. 3.7-4: Cross section excitation functions for the ${ }^{28} \mathrm{Si}(\mathrm{p}, \mathrm{p})$ reaction in the energy region of the second excited $T=3 / 2$ state. 

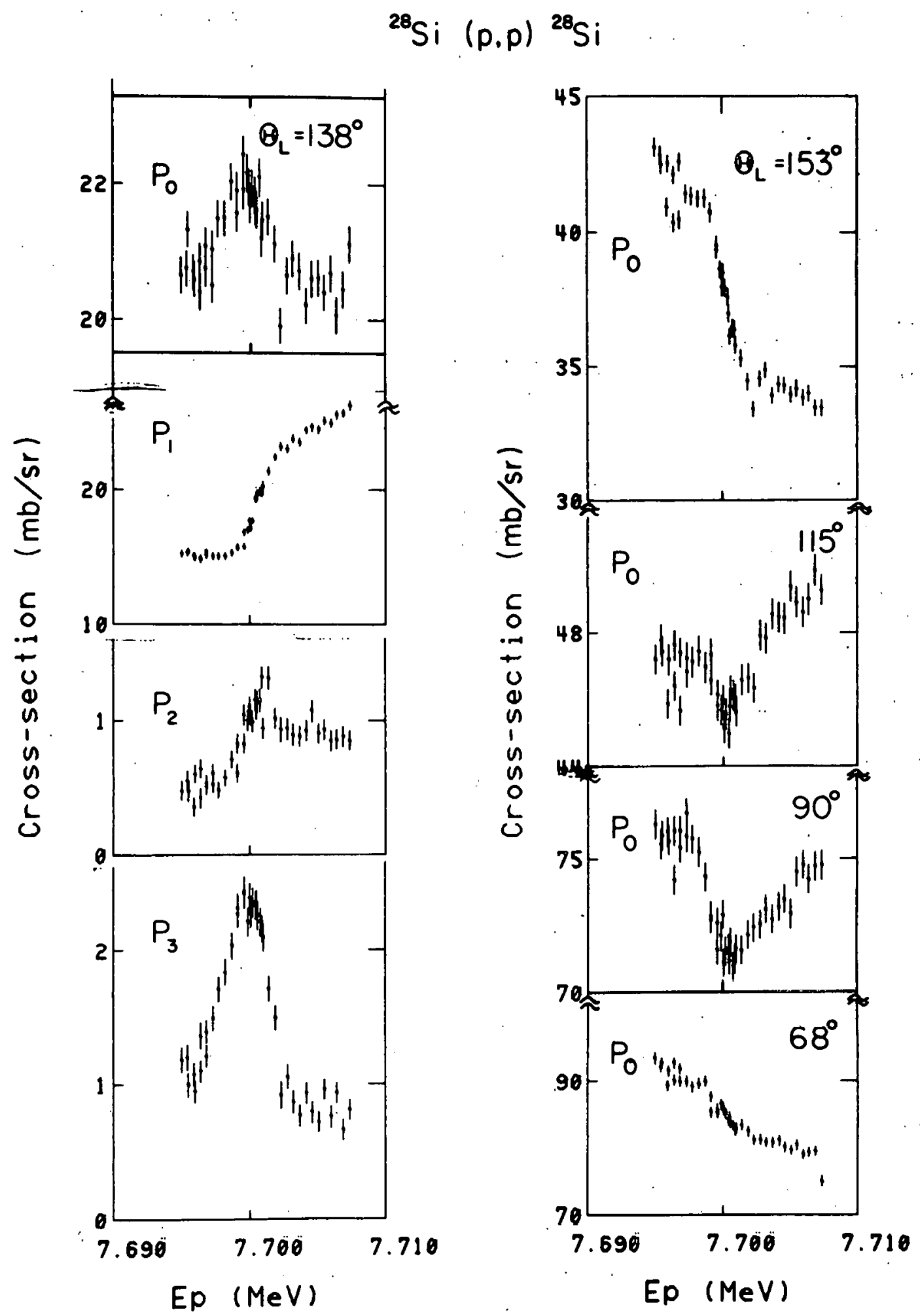

Fig. 3.7-5: Selected excitation functions over the $10.19 \mathrm{MeV}$ state. 
We measured excitation functions in $\sim 0.5 \mathrm{keV}$ steps over this resonance (Fig. 3.7-3). The observed width is $\sim 4 \mathrm{keV}$, a major part of which may be due to the beam energy and target spread. The decay proceeds via the $P_{0}$ and $P_{1}$ branch. No resonance effects were observed in the energetically available $P_{2}$ and $\mathrm{p}_{3}$ channels.

The ratios of $\Delta \sigma / \sqrt{\sigma}$ is approximately constant at all angles, indicating $\mathrm{J} \pi=1 / 2^{+}$. Analysis of these data using procedures similar to those of Ref. 1 will be undertaken soon.

B. The Second Excited $\mathrm{T}=3 / 2$ state in ${ }^{29} \mathrm{P}$

The analog of this state in ${ }^{29} \mathrm{Al}$ has a spin of $7 / 2^{+}$and 1 ies $1.75 \mathrm{MeV}$ above the ground state. Thus in ${ }^{29} \mathrm{P}$ we expect the $7 / 2^{+} \mathrm{T}=3 / 2$ analog to occur around $\mathrm{E}_{\mathrm{x}}=10.13 \mathrm{MeV}$. In a proton scattering experiment the corresponding resonance is expectod at $F_{p}=7.65 \mathrm{MeV}$. We measured excitation function in $1.4 \mathrm{keV}$ steps in the region between $\mathrm{E}_{\mathrm{p}}=7.5$ and $\mathrm{E}_{\mathrm{x}}=7.8 \mathrm{MeV}$ (Fig. 3.7-4). The target for these measurements was $30 \mu \mathrm{g} / \mathrm{cm}^{2}$ thick in ${ }^{28} \mathrm{Si}$. We find that the resonance at $E_{p}=7.701 \mathrm{MeV}\left(E_{x}=10.19 \mathrm{MeV}\right)$ could be the analog of the $T=3 / 27 / 2^{+}$state. Resonance effects were observed in all the proton channels measured at angleo ranging between $55^{\circ}$ and $155^{\circ}$ (Fig. 3.7-5). The interference effect in the elastic scattering data at $90^{\circ}$ indicates positive parity. The absence of a resonance effect at $70^{\circ}$ (the zero of $\mathrm{P}_{4}$ ) and the abrupt change in resonance shape between $115^{\circ}$ and $140^{\circ}$ is consistent with $\ell=4$. Nevertheless, cross section data with better energy resolution (thinner target) and analyzing power measurements are necessary in order to make a firm spin assignment. The observed width of $4 \mathrm{keV}$ could be due mainly to the experimental resolution.

We will be repeating our measurements of excitation function over this state with thinner targets in order to investigate this resonance in more detail. Since the level structure of ${ }^{29} \mathrm{Al}$ up to excitation energies of $3.6 \mathrm{MeV}$ is well known ${ }^{4}$ the study of even higher $\mathrm{T}=3 / 2$ states in ${ }^{29} \mathrm{P}$ could prove very fruitful in establishing the systematics of isospin forbidden widths.

\section{References}

1. P.G. Ikossi et al., Nuc1. Phys. A274, 1 (1976), Phys. Rev. Lett. 36, 1357 (1976).

2. B.M. Skwiersky, C.H. Baglin and D.P. Parker, Phys. Rev. C9, 91 (1974).

3. F. Hinterberger et a1., Nucl. Phys. A263, 460 (1976).

4. P.M. Endt and G. Van Der Leun, Nucl. Phys. A310, 1 (1978). 
8. Gamma Ray Spectrum of the $\beta^{+}$Decay of ${ }^{24} \mathrm{Al}$

E.G. Adelberger and C.D. Hoyle

We have remeasured th $\beta^{+}$decay of ${ }^{24} \mathrm{Al}$ to search for weak branches to high excitation energies in $24 \mathrm{Mg}$. The motivation is to search for transitions which may have large Gamow-Teller matrix elements but have too small branching ratios (due to small phase space) to have been detected in previous work. The ${ }^{24} \mathrm{Al}$ was produced in the reaction ${ }^{24} \mathrm{Mg}(\mathrm{p}, \mathrm{n}){ }^{24} \mathrm{Al}$ using $18 \mathrm{MeV}$ protons. A rabbit system was used to shuttle a natural $\mathrm{Mg}$ target from a bombardment station in cave 1 to cave 2 where the counting was done. Since the half-life of the ${ }^{24} \mathrm{~A} 1$ ground state is $2.07 \mathrm{sec}$, the irradiation time of the target was $\sim 4 \mathrm{sec}$ and the counting time was $\sim 5.5 \mathrm{sec}$ per cycle of the rabbit. The gamma rays were detected with an ORTEC Model 8101-1521W Ge(Li) detector. Between the ${ }^{24} \mathrm{Al}$ and the $\mathrm{Ge}(\mathrm{Li})$ detector was a 1-7/8" 1ucite absorber to stop the positrons with a minimum of bremsstrahlung due to the low $\mathrm{Z}$ of lucite. A lead absorber 3/16" thick was positioned between the $\mathrm{Ge}(\mathrm{Li})$ and the lucite to attenuate low energy gammas.

The high energy portions of two gamma ray spectra are shown in Fig. 3.8-1. Spectrum 1 was accumulated with 0.5 second delay between the end of the irradiation of the target and the beginning of each counting period. Therefore, this spectrum does not contain significant yield of gamma ray lines from the decay of the ${ }^{24} \mathrm{Al}$ m isomer (half 1 ife $=129 \mathrm{~ms}$ ) at $0.44 \mathrm{MeV}$. For spectrum 2, the counting began as soon as the rabbit was in the counting position ( 100 ms after irradiation stopped). This spectrum contains the lines from the decay of the isomer. Identification of the lines is summarized in Table 3.8-1. Peaks 18 and 19 were previously identified, and are in good agreement with this work. Peaks 21 and 22 result from a previously identified decay of the isomer. Peak 20 is new with this work.

Table 3.8-1. Gamma ray lines from the $\beta^{+}$decay of $24 \mathrm{Al}$. An * indicates a line that results from the decay of the ${ }^{24} \mathrm{Al}$ m isomer.

\begin{tabular}{|c|c|c|c|}
\hline Peak No. & $\mathrm{E} \gamma(\mathrm{keV})$ & $\begin{array}{l}\text { Assignment } \\
\mathrm{E}_{\mathrm{i}}-\mathrm{E}_{\mathrm{f}} \text { in }{ }^{24} \mathrm{Mg} \\
\end{array}$ & $\begin{array}{l}2 \text { yield from } \\
2{ }^{4} \mathrm{Al} \text { decay }(\%) \\
\end{array}$ \\
\hline 18 & 7068 & $8436 \rightarrow 1368.6$ & 41 \\
\hline 19 & 7930 & $9298 \rightarrow 1368.6$ & 1.14 \\
\hline 20 & 9442 & $10824 \rightarrow 1368.6$ & 0.16 \\
\hline $21 *$ & 8598 & $9966 \rightarrow 1368.6$ & \\
\hline $22 *$ & 9958 & $9966 \rightarrow 0$ & \\
\hline
\end{tabular}

\section{Reference}

1. Detraz, C., Nuclear Physics, A188, 513 (1972). 

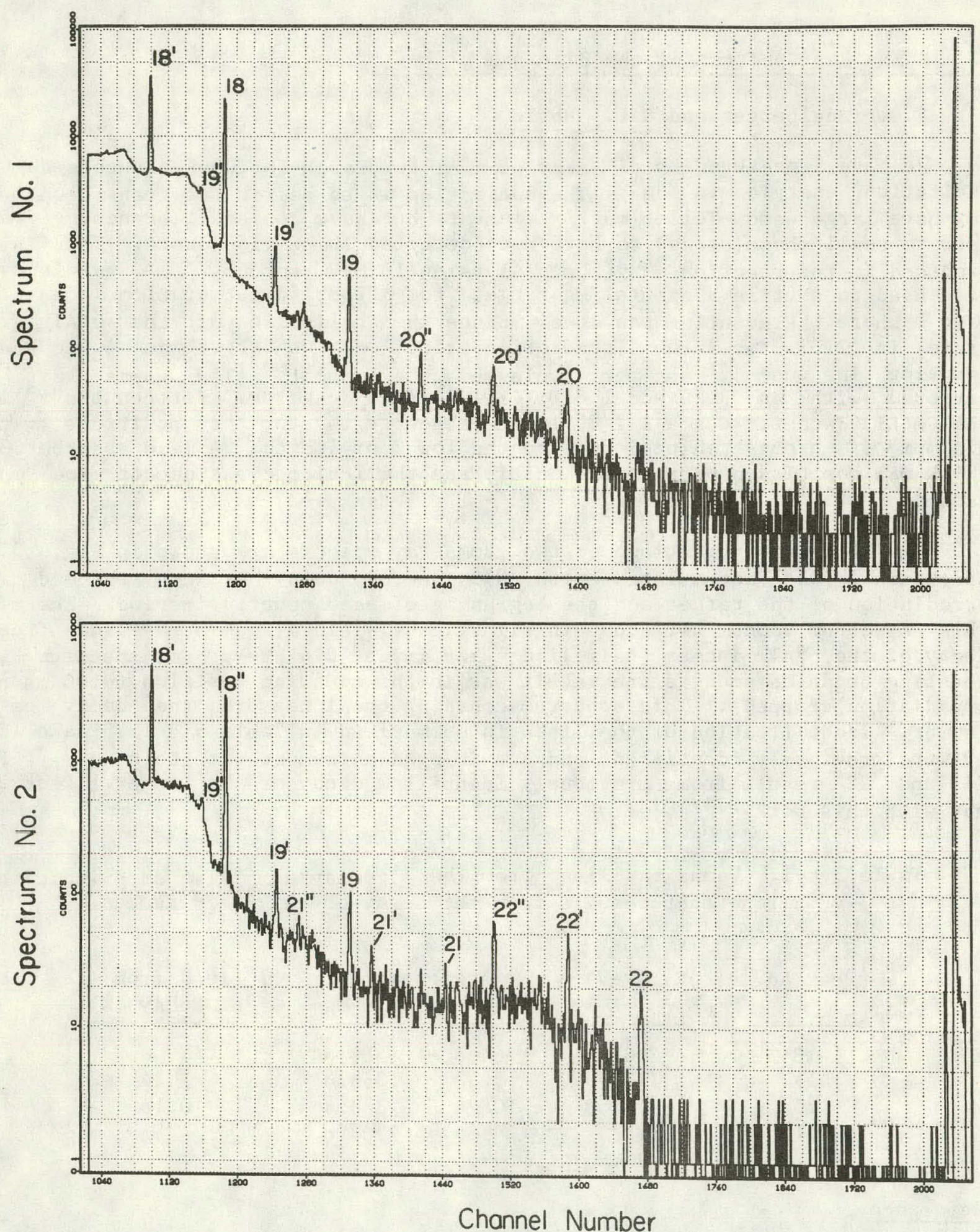

Fig. 3.8-1: Gamma ray spectrum of the $\beta^{+}$decay of $24 \mathrm{Al}$. The primed and double primed peaks are single and double escape peaks, respectively. Gamma ray lines are identified in Table 3.8-1. 


\section{RADIATIVE CAPTURE}

\section{The $12 \mathrm{c}\left(\dot{\mathrm{p}}, y_{\mathrm{O}}\right)^{13} \mathrm{~N}$ Reaction}

A. Unique Determination of the Amplitude and Phase for the Population of the Giant-Dipole Resonance in the Reaction ${ }^{12} \mathrm{C}\left(\mathrm{p}_{\mathrm{pol}}, \gamma_{\mathrm{o}}\right){ }^{13} \mathrm{~N}$

\section{E.G. Adelberger, P.G. Ikossi, K.T. Lesko, and K.A: Snover}

At low energies radiative nucleon capture is dominated by the giant electric-dipole resonance (GDR). Measurements of angular distributions in polarized-proton-capture reactions provide a useful probe of the structure. of the GDR. However, existing polarized-proton-capture studies do not uniquely determine the reaction amplitudes. Even for the most favorable spin sequences, there is an inherent two-fold ambiguity ${ }^{-3}$ which arises from the quadratic nature of the equations relating the amplitudes to the data. We have, for the first time, resolved the two-fold ambiguity in radiative capture by studying the interference between the El "background" and a known Ml resonance. Our results are in agreement with reaction-model calculations and with simple arguments based on the shell model.

The two-fold ambiguity in the case of the reaction $4,51{ }^{12} \mathrm{C}\left(\mathrm{p}_{\mathrm{pol}}, \gamma_{\mathrm{O}}\right){ }^{13_{\mathrm{N}}}$ is illustrated in Fig. 4.1.A-1. The GDR region extends from $\mathrm{E}_{\mathrm{p}} \sim 8$ to $30 \mathrm{MeV}$. $\left(p_{p o l}, \gamma_{0}\right)$ angular distribution results are available ${ }^{5}$ for $E_{p}=16-17 \mathrm{MeV}$. For the simple spin sequence of this reaction, only incoming $s-$ and d-wave amplitudes (with $j=1 / 2$ and $3 / 2$, respectively) contribute to $E 1$ capture. The shell model predicts that d-waves should dominate. Angular distribution measurements can be fitted equally well with two solutions (Fig. 4.1.A-1), one of which is predominantly $d$-wave $(d j)$ and the other predominantly $s$-wave $(s>)$.

We studied the interference between the $E_{p}=14.23 \mathrm{MeV} M 1(E 2) \quad T=3 / 2$ resonance and the $E 1$ GDR. This $T=3 / 2$ resonance ${ }$ is ideal for our purpose since all its important properties ( $\mathrm{J}^{\pi}$, width, strength, mixing ratio, etc.) are well known. 6. The dominant M1-E1 interference effects: should appear in the coefficients $A_{1}$ and $B_{1}$ in the Legendre expansions of the cross section and analyzing power, respectively. Hence we chose to measure excitation curves at $90^{\circ}$ with a polarized beam, and at $55^{\circ}$ and $125^{\circ}$ with an unpolarized beam, where

$$
\begin{aligned}
& 1 / 2\left[\sigma\left(55^{\circ}\right)+\sigma\left(125^{\circ}\right)\right]=A_{0}-0.39 \mathrm{~A}_{4} \sigma_{\text {total }} / 4 \pi, \\
& 1 / 2\left[\sigma\left(55^{\circ}\right)-\sigma\left(125^{\circ}\right)\right]=0.57 \mathrm{~A}_{1}-0.39 \mathrm{~A}_{3},
\end{aligned}
$$

and

$$
\sigma A\left(90^{\circ}\right)=\left[\sigma \uparrow\left(90^{\circ}\right)-\sigma \downarrow\left(90^{\circ}\right)\right] / 2 \mathrm{P}=\mathrm{B}_{1}-1.5 \mathrm{~B}_{3}
$$

Excitation-function datà were measured using a $\sim 200 \mu \mathrm{g} / \mathrm{cm}^{2}$ natural $\mathrm{C}$ target. The polarized-beam data were taken with a $25.4-\mathrm{cm} \times 25.4-\mathrm{cm} \mathrm{NaI}$ spectrometer at $90^{\circ}$ and $\sim 50 \mathrm{nA}$ of polarized proton beam $(\mathrm{P} \sim 0.7)$ from, the 

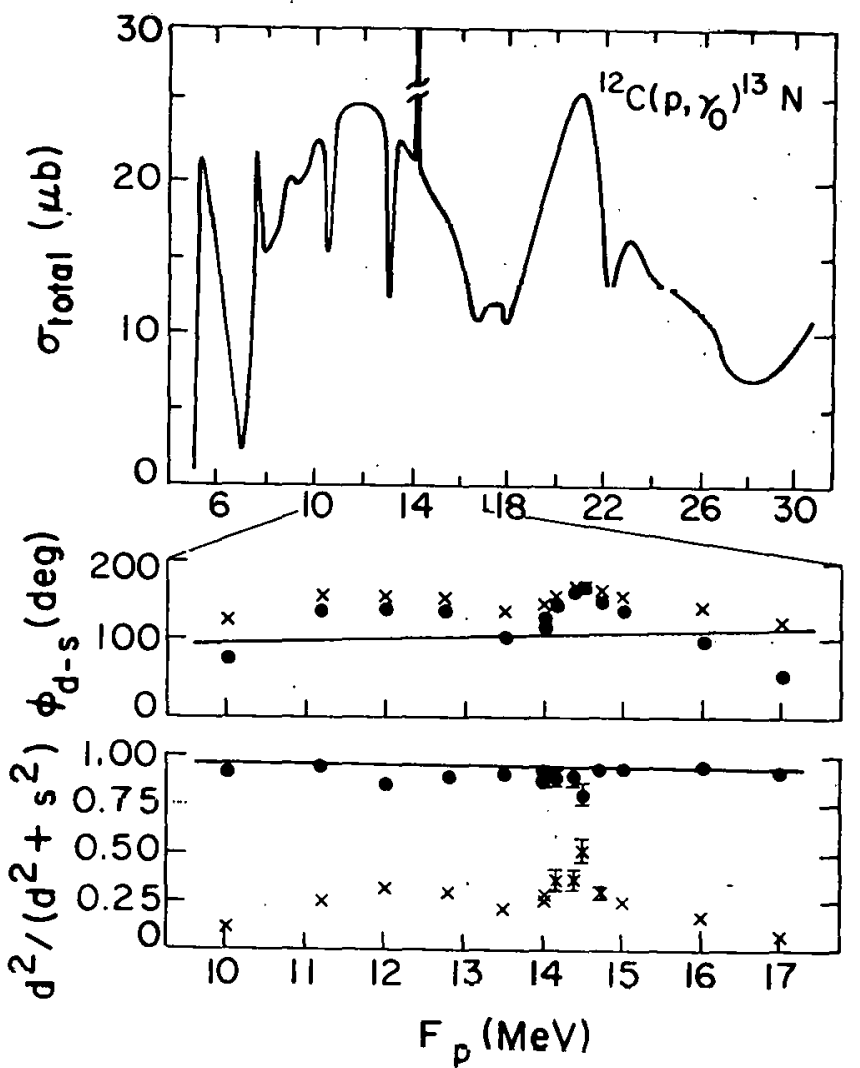

Fig. 4.1.A-1: Upper part: ${ }_{12} \sigma_{\text {total }}$
for ${ }^{2} \mathrm{C}\left(\mathrm{ppol}_{1}, \gamma_{\mathrm{o}}\right)$ for $\mathrm{E}_{\mathrm{p}}={ }_{5-30}$ $\mathrm{MeV}$ (Refs. 4 and 5). Lower part: The $d-s$ phase difference and the relative $d$-wave intensity for $\mathrm{E}_{\mathrm{p}}=10-17 \mathrm{MeV}$ (Ref. 5 plus the present work for $14<\mathrm{E}_{\mathrm{p}}<15$ $\mathrm{MeV})$. The points and crosses correspond to the two different solutions. The solid lines are DSD calculations described in Ref. 5 .

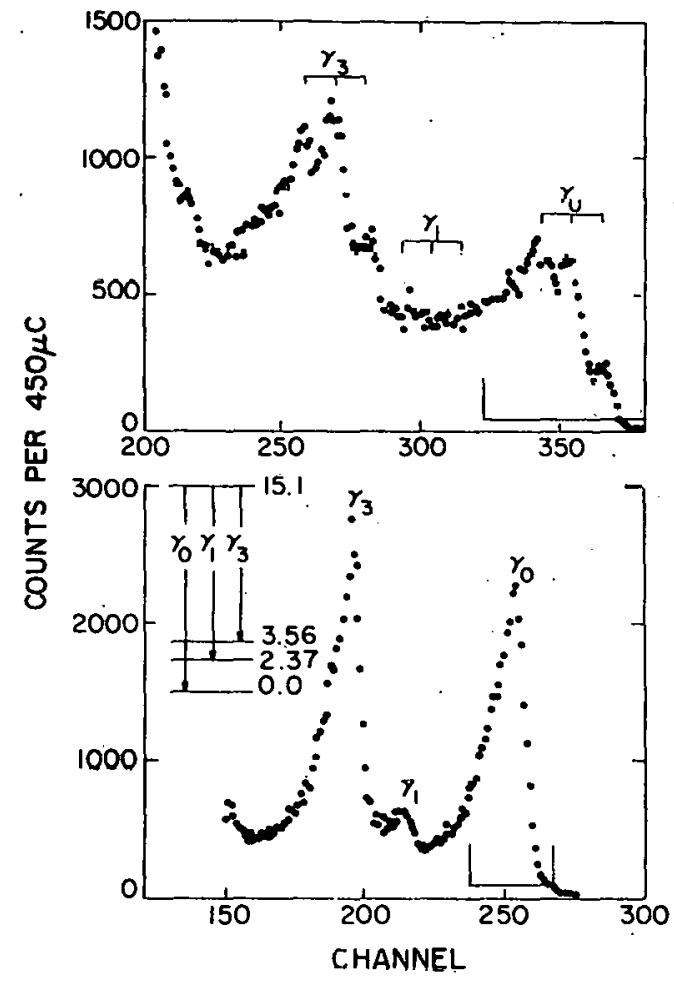

Fig. 4.1.A-2: On-resonance $\gamma$-ray spectra for the large $\mathrm{NaI}$ spectrometer at $55^{\circ}$ (bottom) and the $7.5 \mathrm{~cm} \times 7.5 \mathrm{~cm} \mathrm{NaI}$ at $125^{\circ}$ (top). The vertical bars show the region summed for the $\gamma_{0}$ analysis. 
University of Washington tandem Van de Graaffaccelerator. The unpolarized data (taken with $\sim 350 \mathrm{nA}$ of beam) were measured simultaneously at $55^{\circ}$ and $125^{\circ}$ in order to avoid spurious effects from small accelerator energy shifts. The large $\mathrm{NaI}$ spectrometer was placed at $55^{\circ}$ and a $7.5-\mathrm{cm} \times 7.5-\mathrm{cm} \mathrm{NaI}$ detector at $125^{\circ}$. Representative on-resonance spectra are shown in Fig. 4.1.A-2. The efficiencies of the two detectors were determined from thick-target resonance-yield measurements.

Our resonance measurements for the sum of the cross sections at $55^{\circ}$ and $125^{\circ}$, the $90^{\circ}$ analyzing power $\sigma \mathrm{A}\left(90^{\circ}\right)$, and the difference of the cross sections at $55^{\circ}$ and $125^{\circ}$ are shown in Fig. 4.1.A-3. We fitted these results with a Breit-Wigner resonance (whose width, strength, and E2/M1 mixing ratio are given in Ref. 6) interfering with background $E 1$ and $E 2$ amplitudes determined from a model-independent analysis of our off-resonance polarized-beam angular distributions. The (asymmetric) energy-resolution function and the resonance energy were determined from the shapes of $\sigma\left(90^{\circ}\right)$ and $\sigma\left(55^{\circ}\right)+\sigma\left(125^{\circ}\right)$. $\sigma A\left(90^{\circ}\right)$. was then fitted with the only free parameter being the phase of the M1/E2 resonance relative to the El background. The two bands in Fig. 4.1.A-3 indicate the range of $d_{>}$and $s_{>}$solutions consistent with the off-resonance angular distributions at $E_{p}=14.00,14.17,14.39$, and $14.49 \mathrm{MeV}$. Then the corresponding bands for the difference of the cross sections at $55^{\circ}$ and $125^{\circ}$ were calculated with no free parameters. These bands are insensitive to the E2 parameters, which serve only to determine the off-resonance values for $\sigma A\left(90^{\circ}\right)$ and $\sigma\left(55^{\circ}\right)-\sigma\left(125^{\circ}\right)$ : The results clearly select and $d>$ solution as the physically correct one.

Our experiment has proven that the d) E1 solution is the correct one at $E_{p}=14.23 \mathrm{MeV}$. Furthermore, it is very probable that d-wave capture dominates over the entire region from $E_{p}=10$ to $17 \mathrm{MeV}$ because the two solutions are well separated and nearly independent of energy in this region (see Fig. 4.1.A-1). Although $\left(\mathrm{p}_{\mathrm{pol}}, \gamma\right)$ results are not available for the region $17<\mathrm{E}_{\mathrm{p}}<30 \mathrm{MeV}$ , where the bulk of the El absorption strength lies, the measured ${ }_{4} \mathrm{P}_{2}\left(=\mathrm{A}_{2} / \mathrm{A}_{0}\right)$ suggests that the capture amplitudes here are similar to those in the lower-energy region.

More importantly the ${ }^{12} \mathrm{C}\left(\mathrm{p}_{\mathrm{po} 1}, \gamma_{\mathrm{o}}\right)$ data are very similar to other $1 \mathrm{p}$-she11 capture results such as ${ }^{14} \mathrm{C}\left(\mathrm{p}_{\mathrm{pol}}, \gamma_{\mathrm{o}}\right)$ (Ref. 7) and $13{ }_{\mathrm{N}}\left(\mathrm{p}_{\mathrm{pol}}, \gamma_{\mathrm{o}}\right)$ (Refs. 1 and 3 ) over similar ranges of $E_{p}$ (encompassing the GDR in these latter cases). Thus it is reasonable to infer that $d$-wave dominance is a common feature of $\left(p, \gamma_{0}\right)$ reactions in the upper 1 p shell.

These results provide a basic test of reaction-model calculations such as the direct-semidirect (DSD) mode1 3,7 (see Fig. 4.1.A-1) and the doorway-state mode 18 which predict in the above cases that $d$-wave capture should dominate over s-wave capture, in agreement with our experiment. However, one does not need a detailed calculation to understand the observed $d$-wave dominance. It follows from the basic argument that dipole matrix elements are largest when the initial- and final-state wavefunctions have the same number of nodes. Hence the GDR is expected ${ }^{9}$ to be dominated by nucleon excitations of the form $n \ell \rightarrow n^{-} \ell^{-}$ where $n \ell$ and $n^{-} \ell^{-}$are occupied and unoccupied shell-model orbits, respectively ( $n$ is the principal quantum number, $\ell$ is the orbital angular momentum) and $n^{-}=n$ and $l^{-}=\ell+1$. The same argument leads one to expect that the GDR amplitudes in the $p_{0}$ channe 1 should be dominated by $l^{-}=l+1$ (and $n^{\prime}=n$ ) when 

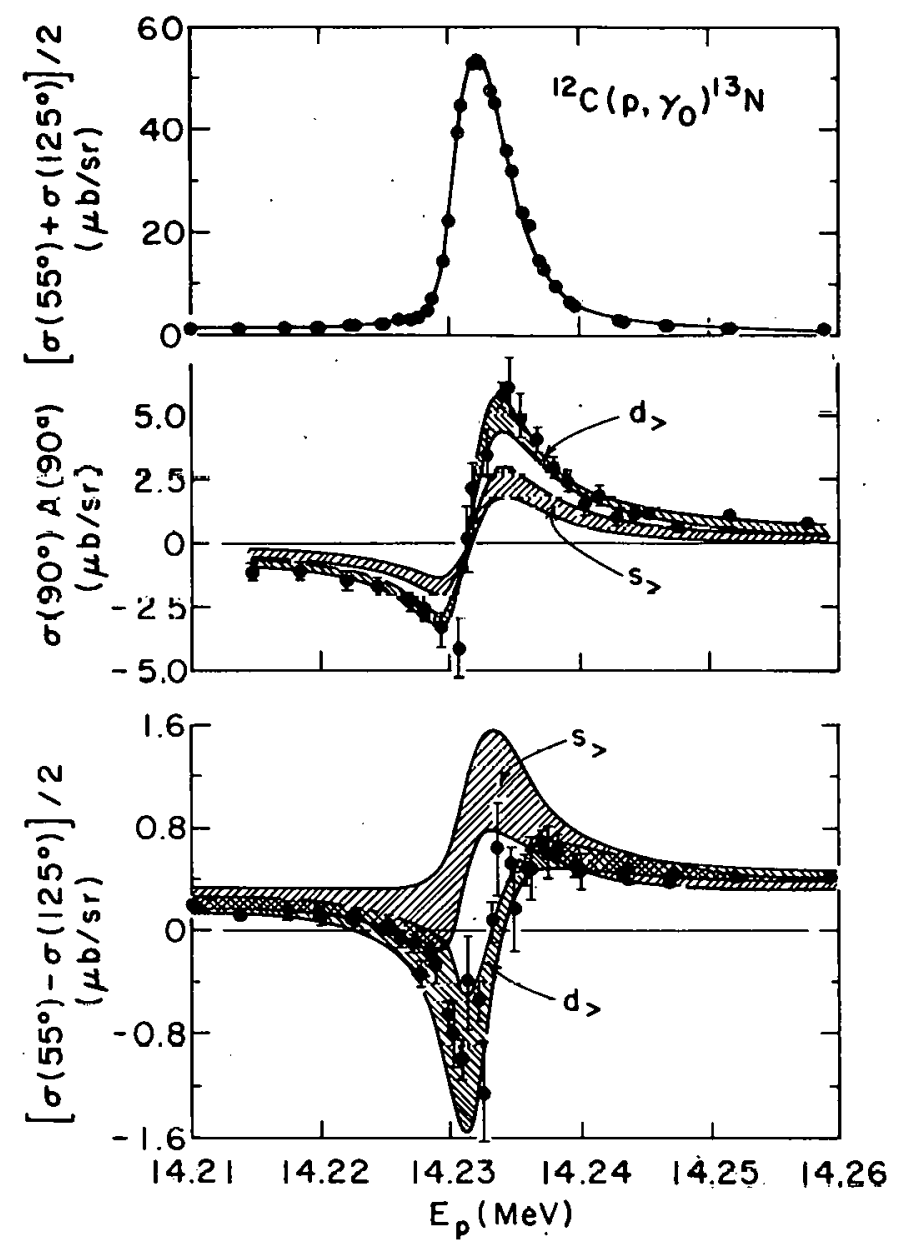

Fig. 4.1.A-3: Excitation curves taken near the lowest $\mathrm{T}+3 / 2$ resonance in ${ }^{12} \mathrm{C}\left(\mathrm{p}, \gamma_{\mathrm{O}}\right)^{13} \mathrm{~N}$. The solid curve in the top part is a calculated f1t. The bands in the lower two parts represent the spread of calculated curves for the $d_{>}$and s> solutions, consistent with the off-resonance angular distributions. 
the target nucleus is related to the final nucleus by the removal of a nucleon from orbital $n \ell$. Since $n=1$ and $\ell=1$ for $1 p$-shell nuclei, d-wave radiative capture should dominate in the GDR.

Our results also provide restrictions on the $\mathrm{E} 2$ contributions to the reaction ${ }^{12} \mathrm{C}\left(\mathrm{p}_{\mathrm{pol}}, \gamma_{\mathrm{o}}\right)$. In Ref. 5 it is shown that if one can neglect background $M 1$ contributions, then small E2 cross sections $\leq 0.5 \mu \mathrm{b}$ (roughly consistent with calculated direct E2 capture) are derived from fits to the angular distribution data. However, at some energies much larger E2 cross sections are also compatible with the data. These solutions with large E2 cross sections have El amplitudes with large s/d ratios ( 0.7$)$. If the El amplitudes are roughly energy independent, then our resonance data reject these large E2 solutions.

More extensive measurements could lead, in principle, to a unique determination of the background $\mathrm{E} 2$ amplitudes and phases near this resonance. Also, this technique can (and should) be applied to other nuclei where $(p, \gamma)$ resonances with known multipolarity occur at sufficiently.high excitation energies to be used for unique determinations of giant-resonance amplitudes and phases.

\section{References}

1. S.S. Hanna, H.F. Glavish, R. Avida, J.R. Calarco, E. Kuhlman, and R. LaCanna, Phys. Rev. Lett. 32, 114 (1974).

2. J.R. Calarco, S.W. Wissink, M. Sasao, K. Weinhard, and S.S. Hanna, Phys. Rev. Lett. 39, 925 (1977); H.R. Weller, N.R. Robertson, and S. Cotanch, Phys. Rev. 18, 65 (1978).

3.. K.A. Snover, in Proceedings of the Third International Symposium on Neutron Capture Gamma Ray Spectroscopy and Related Topics, Brookhaven National Laboratory, 1978 , edited by R.E. Chrien and W.R. Kane (Plenum, New York, 1979), p. 319.

4. D. Berghofer, M.D. Hasinoff, R. Helmer, S.T. Lim, D.F. Measday, and K. Ebisawa, Nuc1. Phys. A263, 109 (1976).

5. R. Helmer, M.D. Hasinoff, J.E. Bussoletti, K.A. Snover, and T.A. Trainor, to be published.

6. R.E. Marrs, E.G. Adelberger, and K.A. Snover, Phys. Rev. C 16, 61 (1977).

7. K.A. Snover, J.E. Bussoletti, K. Ebisawa, T.A. Trainor, and A.B. McDonald, Phys. Rev. Lett. 37, 273 1651(E) (1976).

8. D.G. Mavis, H.F. Glavish, and D.C. Slater, in Proceedings of the Fourth International Symposium on Polarization Phenomena in Nuclear Reactions, Zurich, 19.75, edited by W. Grüebler and V. König (Birkhäuser, Basel, 1976), p. 749.

9. D.W. Wilkinson, Physica (Utrecht) 22, 1039 (1956). 
B. Measurement of $\sigma\left(90^{\circ}\right)$ and $A_{y}\left(90^{\circ}\right)$ for the ${ }^{12} \mathrm{C}\left(p, \gamma_{0}\right)$ Reaction From
$E_{p}=9-14 \mathrm{MeV}$

E.G. Adelberger, P.G. Ikossi, K.T. Lesko, and K.A. Snover

We measured $90^{\circ}$ cross sections and analyzing powers for the ${ }^{12} \mathrm{C}\left(\mathrm{p}, \gamma_{\mathrm{o}}\right)^{13_{\mathrm{N}}}$ reaction in $100 \mathrm{keV}$ steps in the excitation energy range of 10.3 to $14.9 \mathrm{MeV}$. The target was on $1.7 \mathrm{mg} / \mathrm{cm}^{2}$ natural carbon foil. The purpose of the measurements was to search for E2 and $M 1$ strength by looking for resonances in the $90^{\circ}$ analyzing power.

In ${ }^{13} \mathrm{C}$, the mirror nucleus the $\mathrm{E}_{\mathrm{X}}=11.1 \mathrm{MeV}, 1 / 2^{-}$state and the $\mathrm{E}_{\mathrm{X}}=11.8$ $\mathrm{MeV} 3 / 2^{-}$state are knnwn ${ }^{1}$ to decay to the ground state of $13 \mathrm{C}$ via $\mathrm{M} 1$ transitions. One would expect the mirror states in $13_{\mathrm{N}}$ to decay in a similar manner. In lie same energy rcgion in $13 \mathrm{~N}$; states which could decay to the ground state via an $\mathrm{Ml}$ transition occur at $\mathrm{E}_{\mathbf{x}}=10.83 ;\left(1 / 2^{-}\right), \mathrm{E}_{\mathbf{X}}=11.74 \mathrm{MeV}$; $3 / 2^{-}, \Gamma=(530 \pm 80) \mathrm{keV}$ and $E_{x}=11.88 \mathrm{MeV} ;\left(3 / 2^{-}\right)>-(380 \pm 50) \mathrm{keV} .1$

Our analyzing power excitation function (Fig. 4.1.B-1) shows no pronounced structure which would unambiguously establish the presence of an M1 or E2 resonance. However, near $\mathrm{E}_{\mathrm{X}}=11.1$ and $11.8 \mathrm{MeV}$ the analyzing powers show some evidence of structure which should be further investigated. For broad states of the sort listed above, a measurement of $A_{y}\left(90^{\circ}\right)$ as a function of energy may not be sufficient to demonstrate the presence of M1/E2 strength. Detailed measurements of polarized beam angular distributions may be required to make such an identification. It is instructive to note the absence of resonance structure in $\mathrm{A}_{\mathrm{y}}\left(90^{\circ}\right)$ near the minima in $\sigma\left(90^{\circ}\right)$ at $\mathrm{E}_{\mathrm{p}}=10.6$ and $13.1 \mathrm{MeV}$. The presence of destructive interference effects in $\sigma\left(90^{\circ}\right)$ indicates clearly that these two resonances are due to $\gamma$-decays with the same parity as the dominant multipole in the background, i.e., El. In this case, a strong resonance effect in $A_{y}(90)$ is not possible. This is consistent with our data.

\section{References}

1. F. Ajzenberg-Selove, Nuc1. Phys. A268, 1 (1976). 


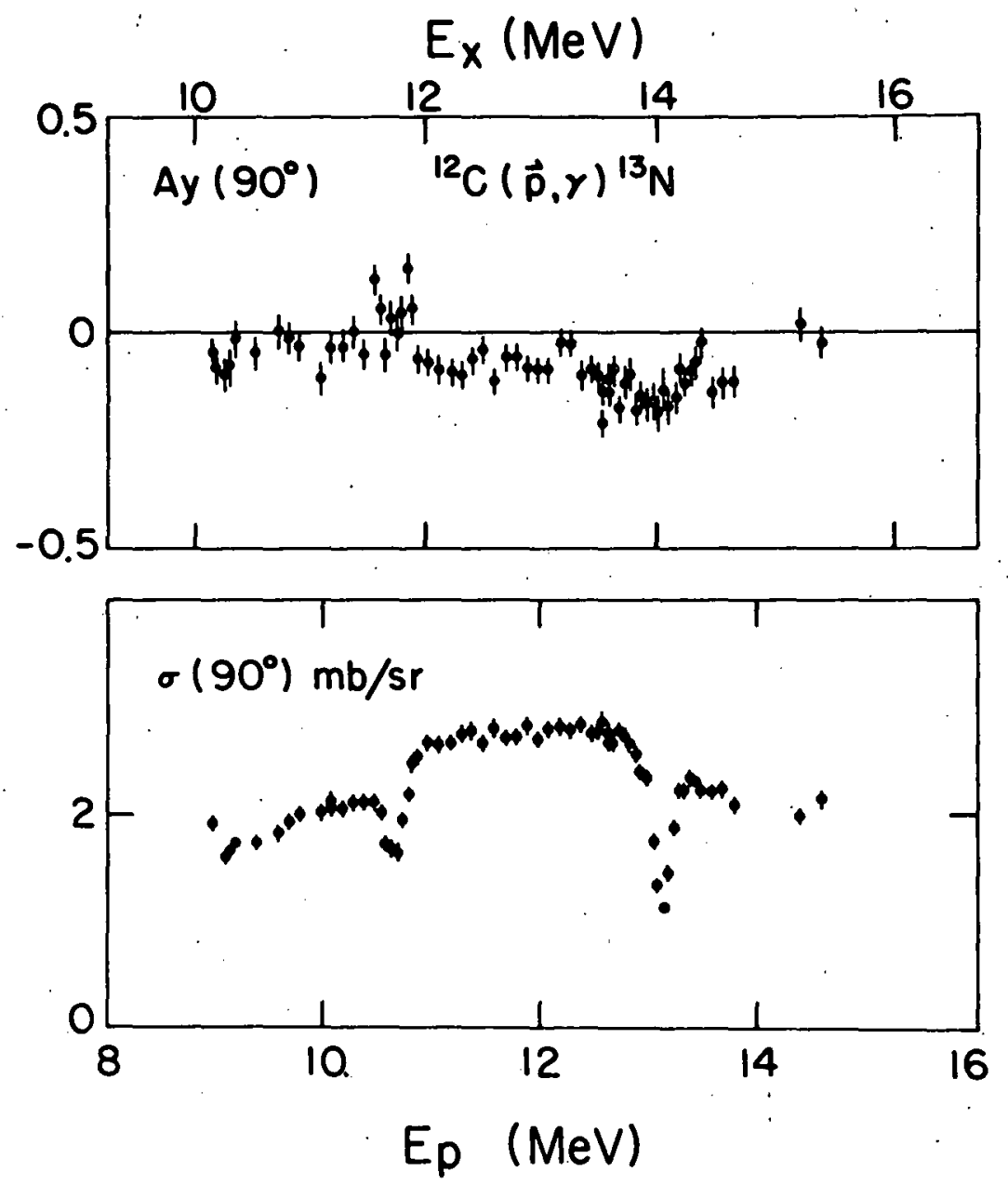

Fig. $4.1 . \mathrm{B}-1:{ }_{12}^{9} 90^{\circ}$ cross section and analyzing power
for the ${ }^{2} \mathrm{C}\left(\mathrm{p}, \gamma_{\mathrm{o}}\right)$ reaction

79 
2. Further Studies of M1 and E2 Resonances in the ${ }^{15_{N}}\left(p, \gamma_{0}\right){ }^{16} 0$ Reaction

P.G. Ikossi, K.T. Lesko, and K.A. Snover

Following our discoveryl of ground-state $M 1$ decays from $I^{+}, T=1$ resonances at $\mathrm{E}_{\mathrm{x}}=16.22,17.14$ and $\sim 18.8 \mathrm{MeV}$ in $16_{0}$, shel1, model calculations were performed ${ }^{2}$ which are consistent with our results and predict substantial fragmented $M 1$ strength at energies up to $28 \mathrm{MeV}_{\text {. }}$ Consequently we have extended our search for ground-state $\mathrm{Ml}$ strength in the $15_{\mathrm{N}}\left(\mathrm{p}, \gamma_{\mathrm{O}}\right) \mathrm{\sigma}_{\mathrm{O}}$ reaction to both higher and lower bombarding energies.

We have measured $\mathrm{A}\left(90^{\circ}\right)$ and $\sigma\left(90^{\circ}\right)$ in $100 \mathrm{kcV}$ steps from $\mathrm{E}_{\mathrm{p}_{15}}=9-16 \mathrm{MeV}$ using a gas cell with a 75 pinch Ni entrance foil and $10^{\prime \prime}$ of $15_{\mathrm{N}_{2}}$ pressure. Relatively suall analyzing powers were found in this energy region: at no energies was $A\left(90^{\circ}\right)>0.2$, and at most energies $A\left(90^{\circ}\right)$ was less than 0.05 . Thus our measurements failed to turn up regions of significant magnitude or structure In $\mathrm{A}\left(90^{\circ}\right)$, consistent with earlier measurements in coarser energy steps. Angular distributions were measured at $\mathrm{E}_{\mathrm{p}}=11.32,11.10,9.73,9.40$, 9.10 , and $8.70 \mathrm{MeV}$, for which good fits $\left(X_{2} \sim 1\right)$ could be obtained assuming a model-independent parameterization in terms of $E 1$ and $E 2$ amplitudes. Hence our results show no evidence for concentrated $M 1$ strength in this energy region. However, Ml resonances in this region (if they exist) may either be too broad or may have too weak a proton formation probability $\Gamma_{p} / \Gamma$ to be observed in $\left(p, \gamma_{0}\right)$. This would not be unexpected at these energies since particle decays to excited $15_{\mathrm{N}}$ (or ${ }^{15} \mathrm{O}$ ) residual states would be favored by the $2 \mathrm{p}-2 \mathrm{~h}$ or $4 \mathrm{p}-4 \mathrm{~h}$ structure of the $1^{+}, T=1$ resonances.

At lower energies we have explored the $E_{p}=1-4 \mathrm{MeV}$ region with polarized and unpolarized beam as shown in Fig. 4.2-1. The 900 cross section shows no evidence for structure in this energy, with the exception of the two well known $1^{-}$resonances at $\mathrm{E}_{\mathrm{p}}<1 \mathrm{MeV}$. More importantly, we find no evidence for a previously reported $3 \mathrm{P}^{+}$resonance at $\mathrm{E}_{\mathbf{X}}=13.68 \mathrm{MeV}$, in agreement with an earlier electron scattering experiment. 4 The data shown near this energy for $\sigma\left(90^{\circ}\right)$ in Fig. 4.2-1 include measurements with proton energy resolution (target thickness) of 20 and $40 \mathrm{keV}$. We obtain $\Gamma_{\gamma_{0}}<1 \mathrm{eV}$ for a resonance near this energy, compared to a previous poor statistics $\left(p, \gamma_{0}\right)$ experiment ${ }^{3}$ which obtained $\Gamma \gamma_{0} \sim 8.5 \mathrm{eV}$. Polarized $\left(\mathrm{p}, \gamma_{0}\right)$ angular distribution results are shown, medsured with $20^{\prime \prime}$ of $15^{\mathrm{N}_{2}}$ gas pressure, corresponding to $170-350 \mathrm{keV}$ energy loss in the target. These data points are plotted at $E_{p}=E_{p}-\Delta E$ (entrance foil) $1 / 2 \triangle \mathrm{E}\left({ }^{15} \mathrm{~N}_{2}\right)$. Polarized beam intensities were $\sim 8-30 \mathrm{nA}$ at these energies, due mainly to the drastic falloff of beam transmission through the tandem at low energics. However, the results clearly show the onset of interesting structure in the odd Legendre coefficients in the $E_{p}=1-3 \mathrm{MeV}$ region, most likely due to a broad $E 2$ resonance (note the strong effect in $a_{3}$ ). $\Lambda$ clear understanding of the parameters for such an E2 resonance, as well as for E2 strength in the $\mathrm{E}_{\mathrm{p}}=$ 4-9 MeV region requires a resonance analysis of the energy dependence of the angular distribution coefficients, which is in progress. 


\section{$\underline{\text { References }}$}

1. K.A. Snover, P.G. Ikossi, and T.A. Trainor, Phys. Rev. Lett. 43, 117 (1979).

2. A. Arima and D. Strottman, Los Alamos Preprint LA-UR-78-2969.

3. C. Rolfs and W.S. Rodney, Nucl. Phys. A235, 450 (1974).

4. M. Stroetze1, Z. Phys. 214, 357 (1968).

Fig. 4.2-1: $\sigma\left(90^{\circ}\right)$ and angular distribution coefficients for the ${ }^{15} \mathrm{~N}\left(\mathrm{p}, \gamma_{\mathrm{o}}\right)^{16} \mathrm{~N}$ reaction

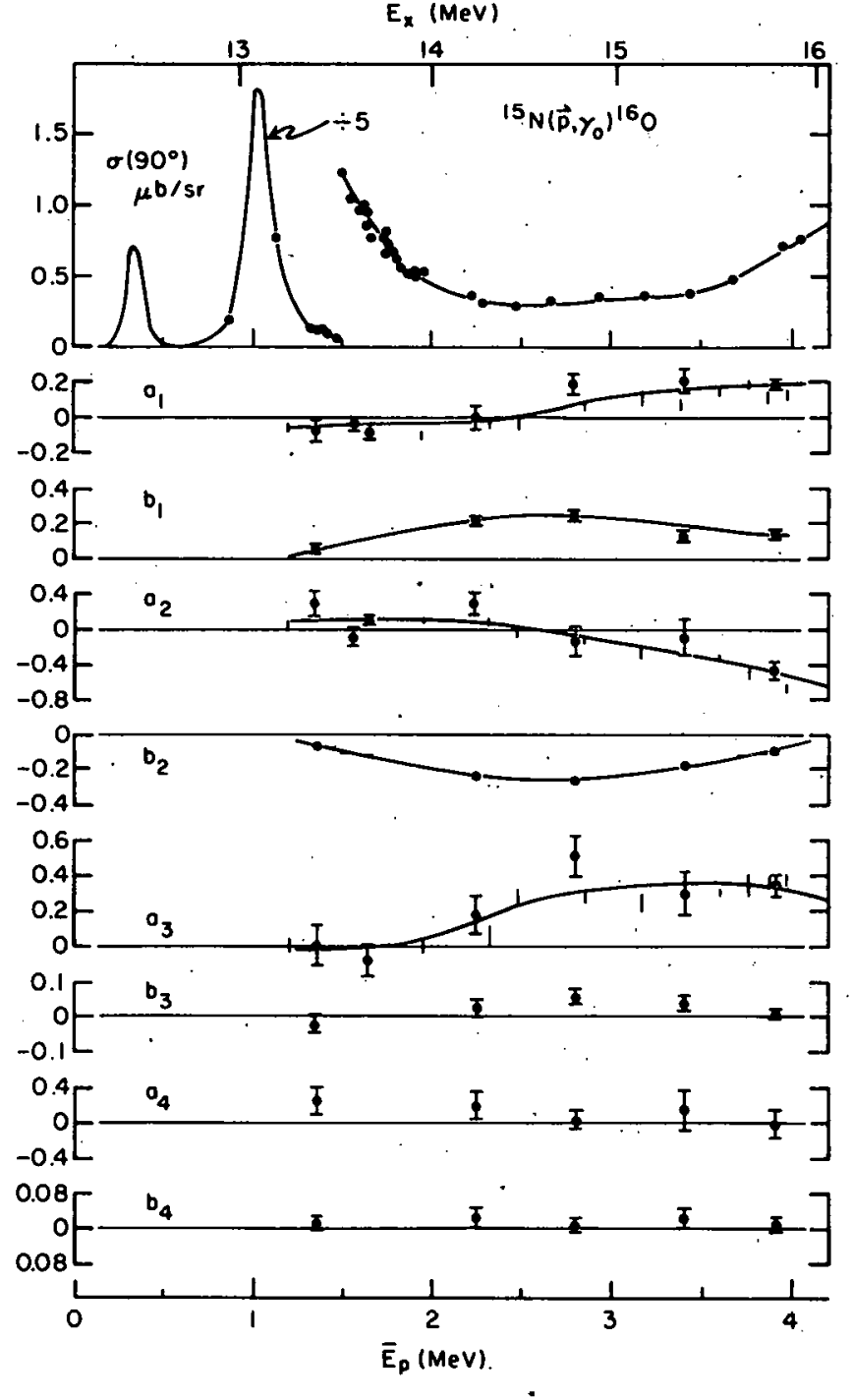


3. Gamma Decay of the $\mathrm{E}_{\mathrm{x}}=10.321 \mathrm{MeV} \mathrm{1}+\mathrm{T}=1$ Level in ${ }^{40} \mathrm{Ca}$

E.G. Adelberger, P.G. Ikossi, W. Rosch, and K.A. Snover

Until recently very little was known about $M 1$ decays in doubly magic $N=Z$ nuclei. Following our discoveryl of substantial M1 decay strength to the ground state of $16_{0}$ a definitive $M 1$ assignment ${ }^{2,3}$ was rade to the well-known $10.321 \rightarrow 0.0 \mathrm{MeV} \gamma$-transition in ${ }^{40} \mathrm{Ca}$ using inelastic electron scattering. This transition is very strong: $\Gamma \gamma_{0}=4.74 \pm 0.30 \mathrm{eV}$, corresponding to $\mathrm{B}(\mathrm{M} 1)=$ $0.37 \mu_{0}^{2}$, which is stronger than the sum of the 3 known Ml transition strengths of $>0.24^{\prime} \mu_{0}^{2}$ to the ground state of $16_{0}$.

l'his level is a well known resonance in the ${ }^{39} \mathrm{~K}(\mathrm{p}, \gamma){ }^{40} \mathrm{Ca}$ reaction at $\mathrm{E}_{\mathrm{p}}=2.043 \mathrm{MeV}$, with a quoted capture strength $\Gamma_{\mathrm{p}} \Gamma_{\gamma} / \Gamma=10.3 \pm 1.7 \mathrm{eV} .4$ This resonance strength has been used as a standard upon which other strength measurements in this mass region are based, 4 and is clearly incompatible with the radiative width quoted above. We have remeasured this resonance strength along with the relative $\gamma$-decay branches to excited final states of ${ }^{40} \mathrm{Ca}$.

He used a target of $\sim 140 \mu \mathrm{g} / \mathrm{cm}^{2}$ of natural potassium iodide $\mathrm{KI}$ evaporated onto 0.1 mil platinum backings upon which a thin layer of gold had been evaporated. Ganma rays were detected in the large NaI spectrometer and in a $15 \%$ $\mathrm{Ge}(\mathrm{Li})$ detector located at $\pm 90^{\circ}$ with respect to the beam axis. Typical beam currents were $\leq 200 \mathrm{nA}$. A troublesome high energy $\gamma$-background present in the early data and due to radiation produced by scattered beam striking the chamber walls was eliminated by stopping the beam immediately downstream of the target in a thin piece of tungsten. Figure 4.3-1 shows the resonance curve for ${ }^{39} \mathrm{~K}\left(p, \gamma_{0}\right)$ obtained in the NaI. The data shows a classic thick-target resonance response, a flat top along with some evidence for a "Lewis-effect" leading edge overshoot. The top half of Fig. 4.3-1 shows the resonance angular distribution for $\gamma_{0}$, which yields $a_{2}=0.09 \pm 0.08$. The NaI efficiency was determined at $E_{y}=15.1 \mathrm{MeV}$ from an earlier ${ }^{12} \mathrm{C}\left(\mathrm{p}, \gamma_{\mathrm{o}}\right)$ resonance yield measurement compared to the absolute strength of Ref. 5, and the relative efficiency between 4.4 and $15.1 \mathrm{MeV}$ was taken from Ref. 6. Assuming additivity to obtain the stopping power for $\mathrm{KI}$ from the tabulated stopping powers ${ }^{7}$ for $\mathrm{K}$ and $\mathrm{I}$, we obtain from the measured $\gamma_{0}$ yield a preliminary value for the capture strength $\Gamma{ }_{p} \gamma_{0} / \Gamma=4.3 \pm 0.4 \mathrm{eV}$.

Branching ratios for resonance decays to excited final states were deduced from an analysis of Ge(Li) spectra accumulated in long runs below, on and above resonance at $\theta y=90^{\circ}$. The $\mathrm{Ge}(\mathrm{Li})$ etficiency was calibrated at low $\mathrm{E} \gamma<3.5 \mathrm{MeV}$ using an absolute ${ }^{60} \mathrm{Co}$ source along with an uncalibrated ${ }^{56} \mathrm{Co}$ source. Absolute $\mathrm{Ge}(\mathrm{Li})$ efficiencies at 4.4 and $10.3 \mathrm{MeV}$ were determined by comparing $\mathrm{Ge}(\mathrm{Li})$ and $\mathrm{NaI}$ yields in the ${ }^{15} \mathrm{~N}(\mathrm{p}, \alpha, \gamma)$ and $39_{\mathrm{K}}\left(\mathrm{p}, \gamma_{\mathrm{o}}\right)$ reactions, along with absolute $\mathrm{NaI}$ efficiencies determined from Refs. 5 and 6 and the ${ }^{12} \mathrm{C}(\mathrm{p}, \gamma)$ measurement discussed above. The small anisotropy observed for the $\gamma_{0}$ transition indicates that the $1^{+}$substates are nearly equally populated, as expected for predominantly s-wave capture. Hence the branching ratios for excited state 


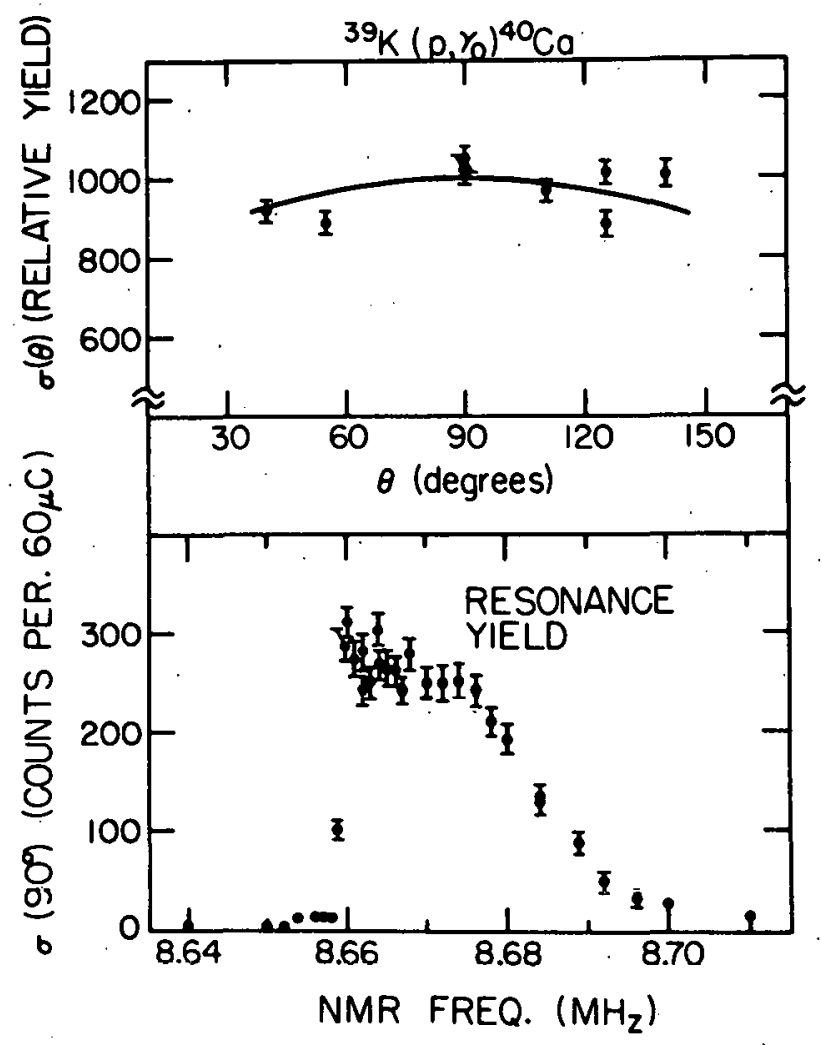

Fig. 4.3-1: UPPER PART: Anguiar distribution for the ${ }^{39} \mathrm{~K}\left(\mathrm{p}, \gamma_{0}\right){ }^{40} \mathrm{Ca}$ reaction on resonance, $f=8.6650 \mathrm{MHz}$. LOWER PART: Resonance yield at $\theta_{\gamma}=90^{\circ}$. 
decays may be reliably estimated from our 900 data. Decays were observed from the resonance $R \rightarrow 0.0 \mathrm{MeV}, R \rightarrow 3.352 \mathrm{MeV}, \mathrm{R} \rightarrow 3.905 \mathrm{MeV}$, and $3.905 \mathrm{MeV} \rightarrow 0.0$ $\mathrm{MeV}$, from which we deduced decay probability (relative to the $R \rightarrow 0.0 \mathrm{MeV} \gamma_{0}$ intensity) of $0.038 \pm 0.004$ and $0.138 \pm 0.009$ for $R \rightarrow 3.352\left(0_{2}^{+}\right)$and $\mathrm{R} \rightarrow 3.905\left(21^{+}\right)$respectively. These strengths correspond to $\mathrm{B}(\mathrm{M} 1$, $\left.1^{+} \rightarrow \mathrm{O}_{2}^{+}\right) / \mathrm{B}\left(\mathrm{M} 1,1^{+} \rightarrow \mathrm{O}_{1}^{+}\right)=0.45 \pm 0.03$ and $\mathrm{B}\left(\mathrm{M} 1,1^{+} \rightarrow 21^{+}\right) / \mathrm{B}\left(\mathrm{M} 1,1^{+} \rightarrow \mathrm{O}_{1}^{+}\right)$ $=0.16 \pm 0.02$, respectively (we assume $\mathrm{E} 2 / \mathrm{M} 1=0$ for $1^{+} \rightarrow 21^{+}$).

It is remarkable that the reduced $B(M 1)$ branching ratios for decays to the excited $\mathrm{O}_{2}^{+}$state relative to the $\mathrm{O}_{1}^{+}$ground state are equal within errors for the three $1^{+}$states studied in doubly magic $\mathrm{N}=\mathrm{Z}$, nuclei (the 16.22 and 17.14 $1^{+}, 1$ states in $16_{0}$ and the $10.321^{+}$, 1 state in ${ }^{40} \mathrm{Ca}$ ).

\section{$\underline{\text { References }}$}

1. K.A. Shuver, P.G. Ikossi, and 'I.A. 'l'rainor, Phys. Rev. Lett. 43 , 117 (1979).

2. W. Gross, D. Meuer, A. Richter, E. Spamer, O. Titze, and W. Knupfer, Phys. Lett. 84B, 296 (1979).

3. P. Burt et al., contributed paper to Internat. Conf. on Nuclear Physics with Electromagnetic Interactions, Mainz, Germany, June 1979 .

4. See, e.g., P.M. Endt, Atomic and Nuclear Data Tables 23, 3 (1979) and references therein.

5. R.E. Marrs, E.G. Adelberger, and K.A. Snover, Phys. Rev. C16, 61 (1977).

6. R.E. Marrs, Ph.D. Thesis, University of Washington, unpublished.

7. C.F. Williamson, J.P. Boujot and J. Picard, Rapport CEA-R3042, Commissariat e $1^{\prime}$ energie atomic.

4. Proton Capture to Excited States in ${ }^{16} 0$

We have measured excitation functions of the $\gamma$-rays resulting from bombardment of $15_{\mathrm{N}}$ by protons in the energy range $2.5-9.5 \mathrm{MeV}$ with emphasis on identifying $M 1$ decays to the first $\left(0^{+}\right)$and second $\left(3^{-}\right)$exrited states in $16_{0}$.

A. M1 $\gamma$-Decay of the 4- $T=1$ Particle-Hole State at $18.98 \mathrm{MeV}$ and the $3^{-}$ $\mathrm{T}=1$ State at $18.03 \mathrm{MeV}$

E.G. Adelberger, P.G. Ikossi, and K.A. Snover

The 4- $\mathrm{T}=1$ state in $16_{\mathrm{O}}$ at $18.98 \mathrm{MeV}$ is particularly interesting since its wavefunction is believed to be predominant $1 \mathrm{y}\left(\mathrm{p}_{3} / \mathrm{f}^{-1}, \mathrm{~d}_{5 / 2}\right)$. A measurement of the strength of its $y$-decay to the $3^{-}(6.13 \mathrm{MeV})$ state yields valuable information on the purity of the $4^{-}, T=1$ shell-model wavefunction. We have studied this state as well as the $3^{-} \mathrm{T}=1$ state at $18.03 \mathrm{MeV}$ as resonances in the $15_{\mathrm{N}}+\mathrm{p}$ entrance channel. Both of these states were previously seen as $\left(p, \gamma_{12}\right)$ resonances.1,2 In a $(p, \gamma)$ coincidence experiment it was shown that the 
observed resonance is due to a decay to the $3-$ state. Using the $(d, t)$ reaction Mairle et al. ${ }^{3}$ assigned $\mathrm{J}^{\pi}=4^{-}$to the $18.98 \mathrm{MeV}$ state and $\cdot \mathrm{J}^{\pi}=3^{-}$to the 18.03 $\mathrm{MeV}$ state. The same authors, ${ }^{4}$ using coincidence techniques, measured the particle decays of these states.

In Fig. 4.4.A-1 we present excitation functions measured at $90^{\circ}$ using polarized protons. The data below $7.4 \mathrm{MeV}$ in this figure were, taken with a $15_{\mathrm{N}}^{\circ}$ ( $99.9 \%$ purity) gas target $\left(0.3 \mathrm{mg} / \mathrm{cm}^{2}\right)$ with $\mathrm{Ni}$ entrance $\left(0.6 \mathrm{mg} / \mathrm{cm}^{2}\right)$ and exit foils. At higher energies a thicker ${ }^{15} \mathrm{~N}$ target $\left(0.64 \mathrm{mg} / \mathrm{cm}^{2}\right)$ and thicker $\mathrm{Ni}$ foils $\left(0.9 \mathrm{mg} / \mathrm{cm}^{2}\right)$ were used. The high energy $\gamma$-rays following the proton capture were detected with the $24 \times 24 \mathrm{~cm} \mathrm{NaI}$ spectrometer with anticoincidence shield. These spectra were used to obtain information of the $\gamma_{0}$ and $\gamma_{12}$ decays. The $\gamma_{34}$ transitions occur in an energy range where there were also $\gamma$-rays from the $\mathrm{Ni}$ foil. Additional data on the $\mathrm{E}_{\mathrm{x}}=18.98 \mathrm{MeV}$ and $\mathrm{E}_{\mathrm{x}}=18.03 \mathrm{MeV}$

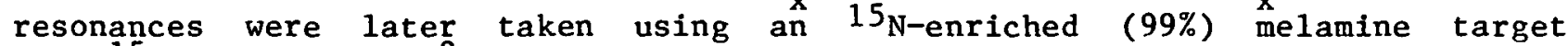
$\left(\mathrm{C}_{3} \mathrm{H}_{6}{ }^{15} \mathrm{~N}_{6} \sim 0.29 \mathrm{mg} / \mathrm{cm}^{2}\right)$. The spectra obtained with the melamine targets allowed extraction of the $\gamma_{34}$ cross-sections. The lower energy part of the spectrum prescaled by a factor of ten and without the anticoincidence requirement was digitized at the same time as the normal high energy portion. This provided information on the $\gamma$-rays from the $\alpha_{1}, p_{12}$, and $p_{3}$ exit channels.

The areas were extracted by performing lineshape fits to the data. The lineshapes were empirically deduced from the data. No background subtraction was necessary for the capture $\gamma$-rays. For the reaction $\gamma$-ray spectra an empirical background lineshape determined at bombarding energies below the $p_{12}$ threshold was used. Typical spectra with the corresponding fits are shown in Fig. $4.4 \cdot \mathrm{A}-2$.

In all the fits the relative separation of the observed lines was fixed by the known energy differences of the final states. The width of the lineshape for the capture $\gamma$-rays was assumed to increase linearly with energy whereas for the reaction $\gamma$-rays the width was kept constant. The absolute normalization ( \pm $10 \%$ ) of the data was deduced by normalizing the $\gamma_{0}$ yields to our previously measured $^{5}$ absolute cross-section at $7.3 \mathrm{MeV}$. $\AA 3 \%$ per $\mathrm{MeV}$ correction to the data was performed to account for the change in the accept efficlency as a function of energy. The agreement between our present $\gamma_{0}$ data and those of Ref. 5 is very good. The $\gamma_{0}$ and $\gamma_{12}$ data are also in good agreement with those of Refs. 1 and 2. To obtain the normalization of the reaction $\gamma$-rays we used information on the relative efficiency of the $\mathrm{NaI}$ at 4.4 and $15.1 \mathrm{MeV}$ for the accept and reject spectra extracted from Ref. 6. This procedure was followed independently for the gas-cell and melamine target data. The resulting cross-section for the $4.4 \mathrm{MeV} \gamma$-rays thus obtained at $\mathrm{E}_{\mathrm{p}_{2}} \sim 6.3$ and $\mathrm{E}_{\mathrm{p}} \sim 7.3$ are in good agreement indicating that the contribution from ${ }^{12} \mathrm{C}\left(\mathrm{p}, \mathrm{p}^{-} \gamma\right)$ is small at these energies. However the off resonance cross-sections obtained for the 5.3 $\mathrm{MeV} \gamma$-rays for $\mathrm{E}_{\mathrm{p}} \sim 7.3 \mathrm{MeV}$ differ in the melamine and the gas cell data by $\sim 20 \%$. Resonance strengths deduced from the melamine and the gas cell data are consistent, indicating the higher yield of $5.3 \mathrm{MeV} \gamma$-rays in the gas cell data is due to some contaminant background near $5.3 \mathrm{MeV}$ which is present in the gas-cell data and absent in the melamine data. All data used to determine resonance strengths were internally consistent, and have a $+10 \%$ uncertainty in absolute normalization. 


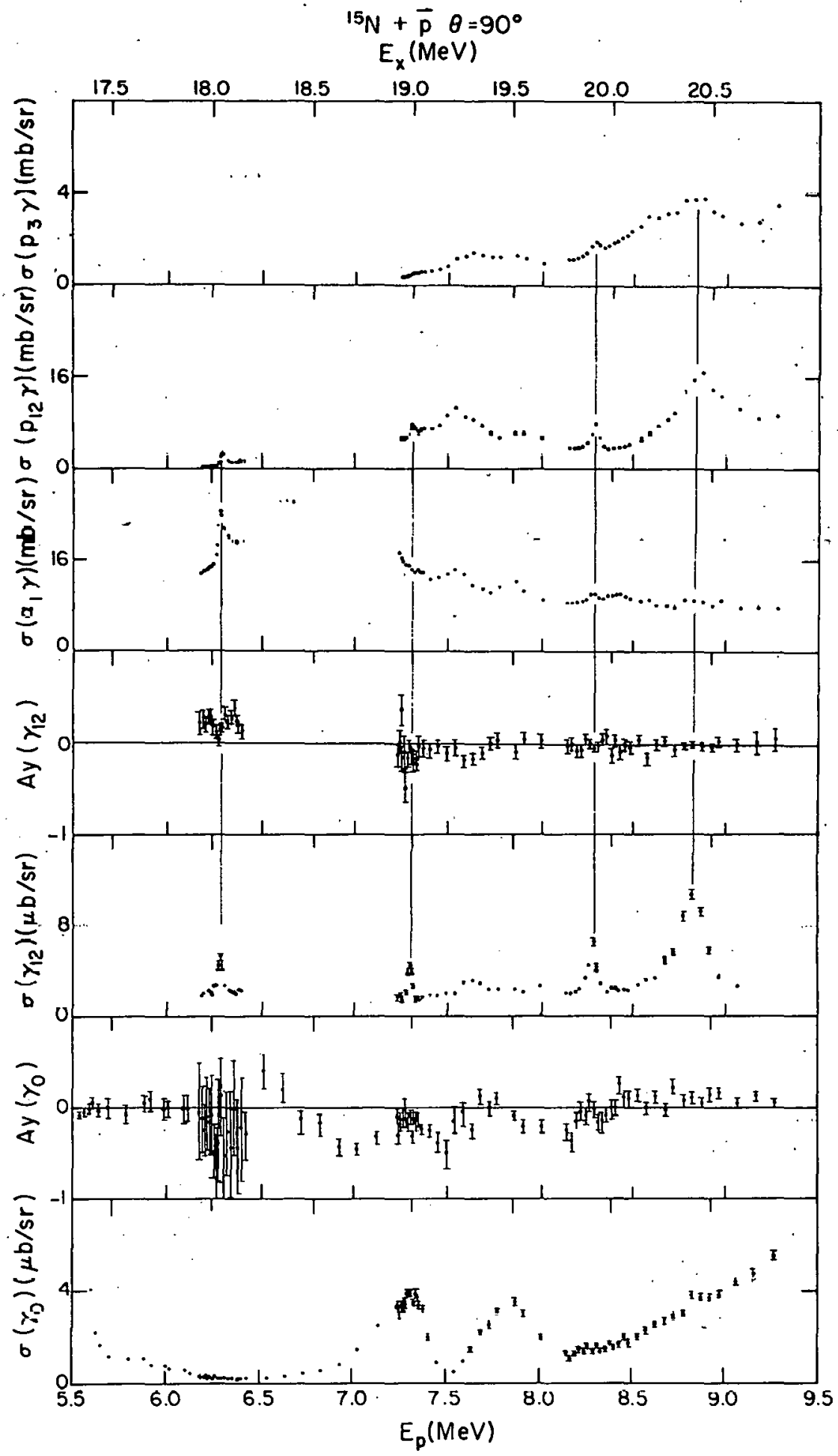

Fig. 4.4.A-1: Excitation functions for the ${ }^{15} \mathrm{~N}\left(\mathrm{p}, \gamma_{0}\right)^{16} \mathrm{O},{ }^{15} \mathrm{~N}\left(\mathrm{p}, \gamma_{12}\right)^{16} \mathrm{U}$ $\left(E_{X}=6.05,6.13 \mathrm{MeV}\right),{ }^{15} \mathrm{~N}\left(\mathrm{p}, \alpha_{1} \gamma\right)^{12} \mathrm{C},{ }^{15} \mathrm{~N}(\mathrm{p}, \mathrm{p} 12 \gamma){ }^{15} \mathrm{~N}$, and ${ }^{15} \mathrm{~N}\left(\mathrm{p}, \mathrm{p}_{3} \gamma\right)^{15} \mathrm{~N}$ measured using a gas target. 


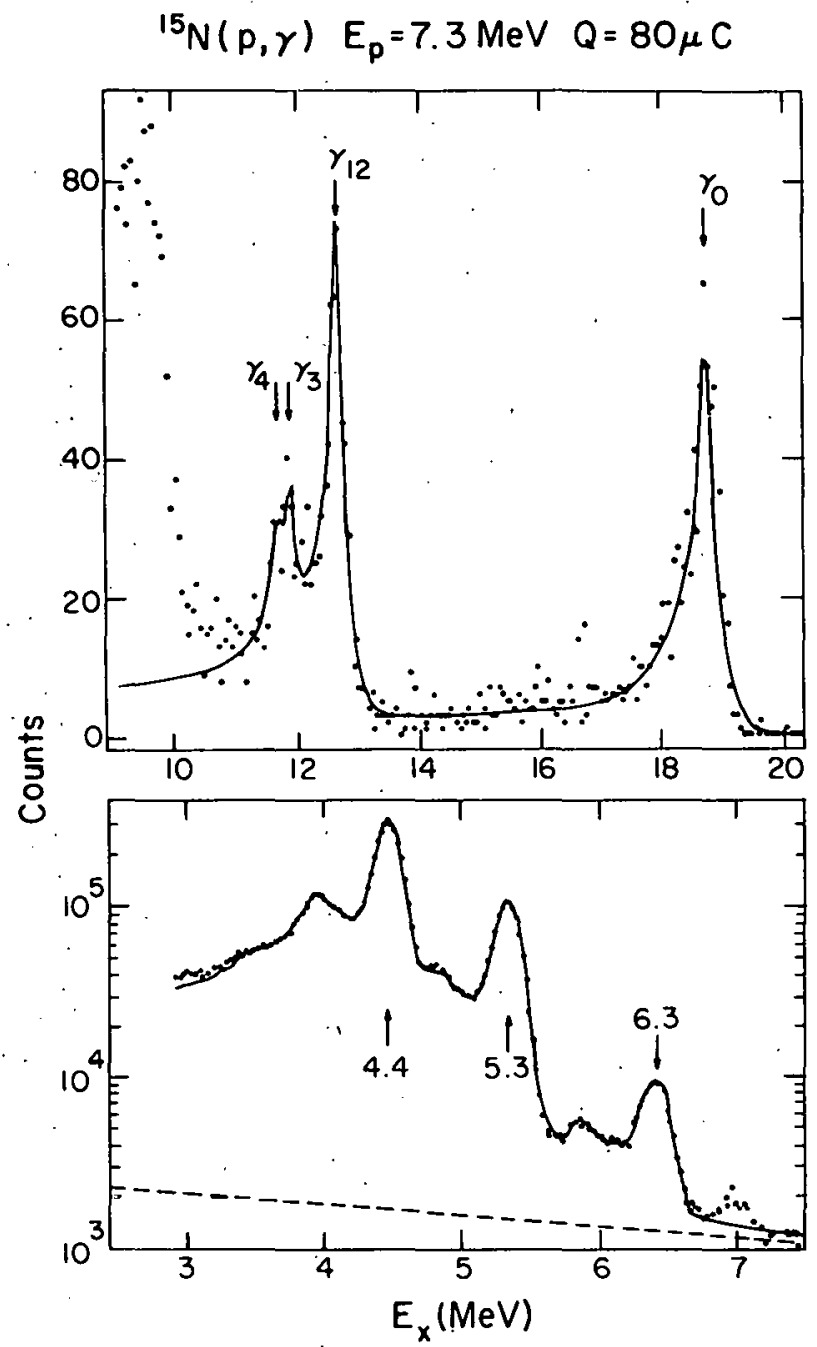

Fig. 4.4.A-2: Typical spectra obtained with a melamine target. The solid lines are the result of a line shape fit used in the area extraction.

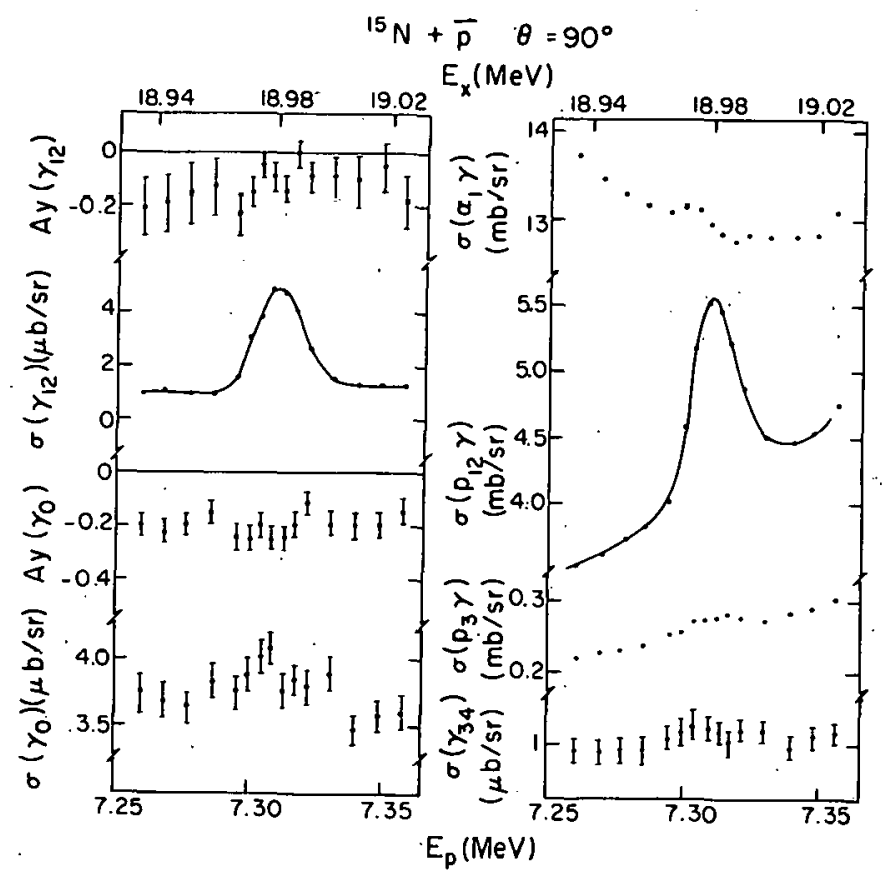


Our more detailed excitation function over the $18.98 \mathrm{MeV}$ and $18.03 \mathrm{MeV}$ states are shown. in Figs. 4.4.A-3 and -4 , respectively. The $\gamma_{12}$ analyzing power data over these resonances show little evidence of Ml-E1 resonance-background interference. Although the presence of analyzing powers at 900 would be a definite indication that these states decay via M1 or E2 transitions, its absence does not exclude this possibility. This is due to the uncertainty in the nature of the background cross-section. If one assumes that the off-resonance $\gamma_{12}$ cross-section is due primarily to E1 decays to the $3^{-}$ state then it may contain contributions from $2^{+}, 3^{+}$, and $4^{+}$initial states. Thus for a $3^{-}$state populated by $d_{5 / 2}$ and $g_{7 / 2}$ entrance partial waves, one obtains 10 terms contributing to $b_{1}$, so that strong cancellations are possible.

Our angular distribution measurements for $\gamma_{12}$ near the $18.03 \mathrm{MeV}$ state (Fig. 4.4.A-5) yip1d $a_{2}$ (resnnance) $=0.55 \pm 0.06$ and $a_{1}, a_{3}, a_{4}$ consistent with zero. This is consistent with pure dipole decay. Assuming pure dipole decay, the a coefficient restricts the angular momentum of this state to $\mathrm{J}=3$, for which $0.375<a_{2}<0.50$, in agreement with Ref. 1. The measured a given above suggests the presence of both $\mathrm{d}_{5 / 2}$ and $g_{7 / 2}$ entrance channels, with $\mathrm{d}_{5 / 2} / \mathrm{g}_{7 / 2} \sim 0.9$, assuming negative parity as assigned in Ref. 3. Our measured resonance strengths (Table 4.4.A-1) for the $\mathrm{p}_{12}$ and $\alpha_{1}$ channels are consistent with the branching ratios of Ref. 4 and confirm the identification of this resonance as the same level populated in the direct reaction studies of Refs. 3 and 4. Similarly, for the $E_{x}=18.98 \mathrm{MeV}$ we find $a_{2}=-0.284 \pm 0.114$ and again $a_{1}, a_{3}, a_{4}$ consistent with zero, indicating pure dipole decay- The theoretical limits for the dipole decay of a $\mathrm{J}=4$ resonance are $-0.304<\mathrm{a}_{2}<-0.357$ and for $J=2-0.0715<a_{2}<0.143$. Hence our measurement favors a $J=4$ assignment, in agreement with Ref. 1. Again the negative parity assignment follows from Ref. 3. The results are summarized in Table 4.4.A-1. The absolute widths are determined from combinations of our strength measurements and the branching ratios of Ref. 4. The uncertainty in the absolute widths is dominated by the uncertainties in the branching ratios.

$\Lambda$ shell model calculation of Millener assuming a $1 \mathrm{p}-1 \mathrm{~h}$ basis for the $4^{-}$ states gives $\Gamma_{\gamma_{2}}=10 \mathrm{eV}$ for the $4^{-}, 1 \rightarrow 3,0^{\prime}$ decay strength, in agreement with the measured value.

\section{References}

1. S.H. Chew, J. Lowe, and J.M. Ne1,son, Nuc1. Phys. A286, 451 (1977).

2. S.H. Chew, J. Lowe, and J.M. Nelson, Nucl.: Phys.. $\overline{\text { A229 }}, 241$ (1974).

3. G. Mairle, G.J. Wagner, P. Doll, K.T. Knöpfle, and H.Breuer, Nucl. Phys. A299, 39 (1977).

4. H. Breuer, P. Doll, K.T. Knöpfle, G. Mairle, and G.J. Wagner (to be published).

5. K.A. Snover, P.G. Ikossi, and T.A. Trainor, Phys. Rev. Lett. 43, 117 (1979).

6. R. Marrs, Ph.D. Dissertation, University of Washington (1975).

7. Millener, private communication. 


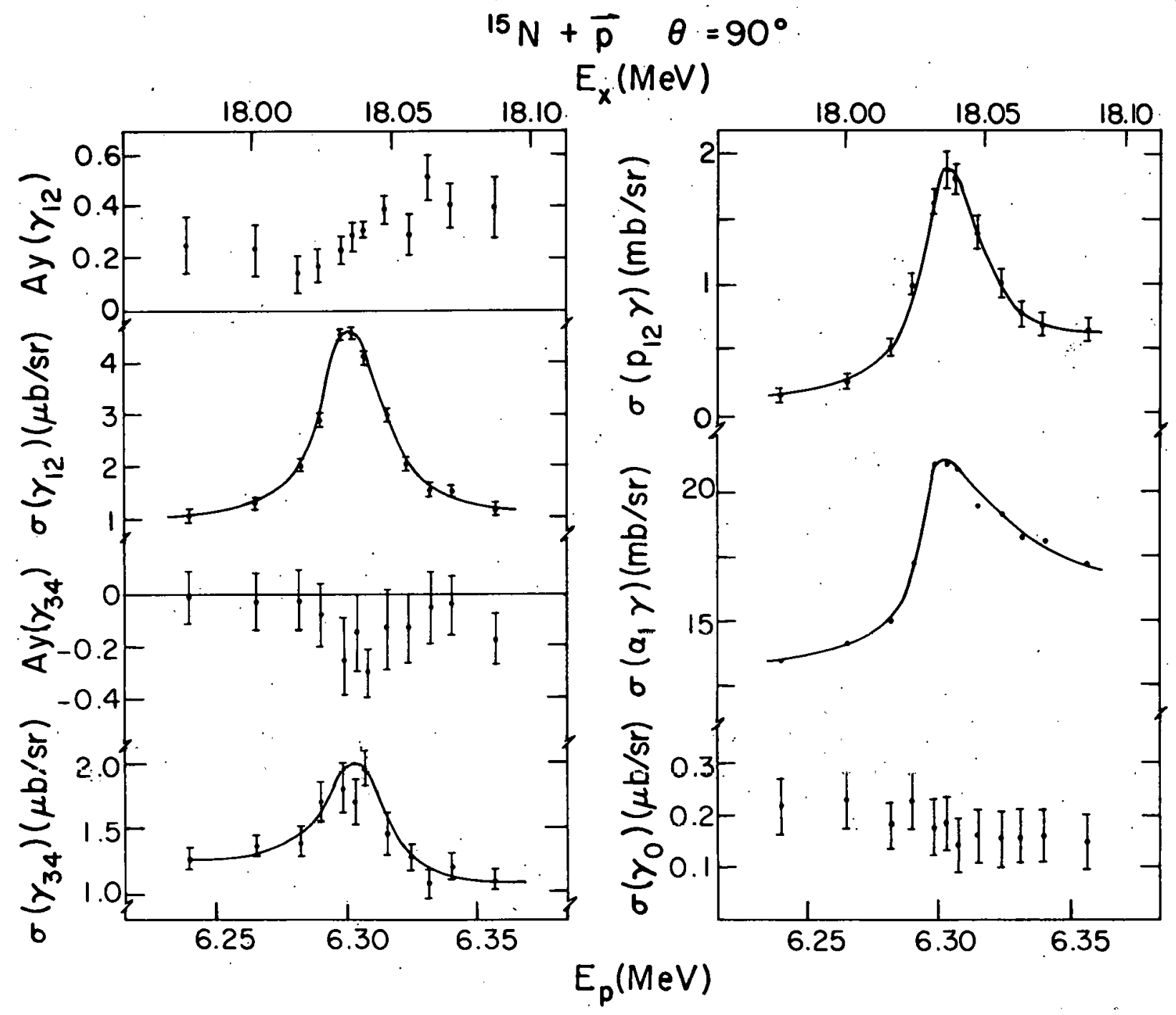

Fig. 4.4.A-4: Excitation functions in the vicinity of the $18.03 \mathrm{MeV}$ $3^{-}$state taken with a melamine target. 


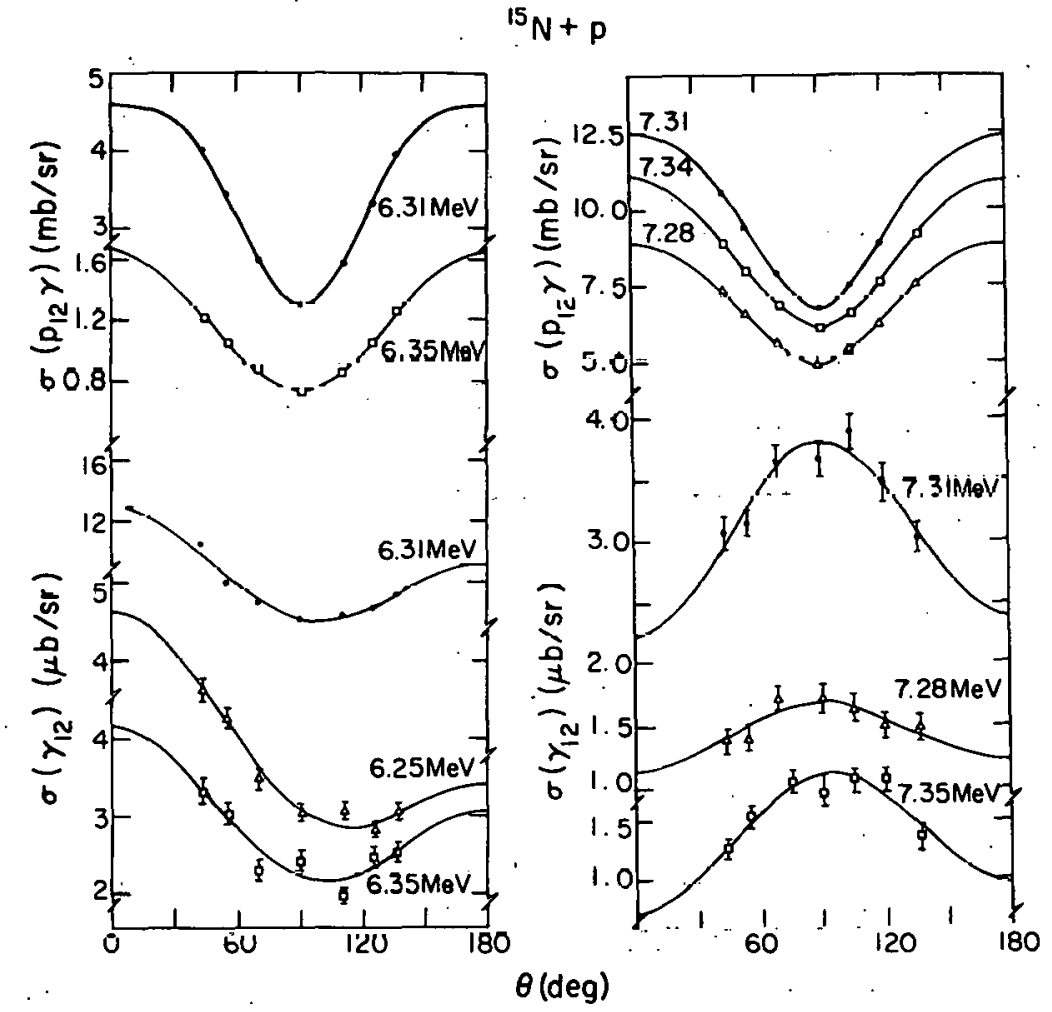

Fig. 4.4.A-5: Angular distributions for the ${ }^{15} \mathrm{~N}\left(\mathrm{p}, \gamma_{12}\right)$ and ${ }^{15} \mathrm{~N}\left(\mathrm{p}, \mathrm{p}_{12} \gamma\right)$ cross section in the vicinity of the 18.03 and $18.98 \mathrm{MeV}$ states. The data close to $18.98 \mathrm{MeV}$ were taken with a gas target, the rest with a melamine target. 
Table 4.4-1: Properties of High Spin "Particle-Hole" States in ${ }^{16} 0$

$E_{p}=6.31 \pm 0.01$

(a)

$\frac{\Gamma_{P} \Gamma_{P_{12}}}{\Gamma}=(1.105 \pm 0.26) \mathrm{keV}$

$\frac{\Gamma_{p}{ }^{r} \alpha_{1}}{\Gamma}=(4.25 \pm 1.00) \mathrm{keV}$

$\frac{{ }^{\Gamma}{ }_{p} \gamma_{12}}{\Gamma}=(1.96 \pm 0.27) \mathrm{eV}$.

$\frac{{ }^{r}{ }^{\Gamma} \gamma_{34}}{\Gamma}=(0.31 \pm 0.11) \mathrm{ev}$

$E_{p}=7.31 \pm 0.01$

(a)

$\frac{\Gamma_{p}{ }^{r} p_{12}}{\Gamma}=(0.618 \pm 0.086) \mathrm{keV}$

$\frac{\Gamma_{p} \Gamma_{\alpha}}{\Gamma} \leq(0.066 \pm 0.021) \mathrm{keV}$

$\frac{\Gamma_{p}{ }^{\Gamma} \alpha_{12}}{\Gamma}=0.85 \pm 0.10 \mathrm{eV}$

$\frac{\Gamma_{p}{ }^{\Gamma} \gamma_{34}}{\Gamma} \leq 0.03 \mathrm{eV}$

$$
E_{x}=18.03 \quad J^{\pi}=3^{-}
$$

(b)

(d)

$$
r_{p} j r=0.41 \pm 0.15
$$$$
\Gamma_{T}=23 \pm 72 \mathrm{keV}
$$

$\Gamma_{a_{1}} / \Gamma=0.46 \pm 0.15$

(c)

$$
\Gamma_{p_{0}}=(9.2 \pm 3.7) \mathrm{keV}
$$

$a_{2}\left(\gamma_{12}\right)=0.55 \pm 0.06$

$$
r_{p_{12}}=(2.7 \pm 1.2) \mathrm{kev}
$$

$a_{2}\left(p_{12}\right)=0.83 \pm 0.01$

$\Gamma_{\alpha_{1}}=(10.4 \pm 4.5) \mathrm{keV}$

$a_{2}\left(\alpha_{1}\right)=-0.41 \pm 0.15$

$\Gamma_{\gamma_{12}}=(4.8 \pm 1.9) \mathrm{eV}$

$\Gamma_{\gamma_{34}}=(0.76 \pm 0.39) \mathrm{eV}$

$E_{x}=18.98 \quad J^{\pi}=4^{-}$

(b)

(d)

$$
\Gamma_{\mathrm{P}_{12}} / \Gamma=0.63 \pm 0.08
$$

$\Gamma=(8.2 \pm 3.8) \mathrm{keV}$

$\Gamma_{p} / \Gamma=0.12 \pm 0.05$

(e)

$$
r_{\gamma_{12}}=(7.1 \pm 3.1) \mathrm{eV}
$$

$a_{2}\left(\gamma_{12}\right)=-0.284 \pm 0.114$

$r_{34}<0.3 \mathrm{eV}$

$a_{2}\left(p_{12}\right)=0.7 .19 \pm 0.001$
$\Gamma_{P_{1} ?}=(5.2 \pm 2.3) \mathrm{keV}$

$\Gamma_{\alpha_{1}} \leq(0.55 \pm 0.26) \mathrm{keV}$

$r_{p}=(0.98 \pm 0.19) \mathrm{kev}$

(a) Present results

(b) Ref, 3

(c) Present results

(d). From combining (a) and (b) 
B. M1 $\gamma$-Decay of the $1+16.22$ and $17.14 \mathrm{~T}=1$ States to the Excited $0^{+}(6.05$

MeV) Final State in $16_{0}$

\section{E.G. Adelberger, P.G. Ikossi, and K.A. Snover}

With the use of ${ }^{15} \mathrm{~N}$-enriched solid melamine targets as described in the previous section, we were able to obtain reliable measurements of the $\gamma_{12}$ and $\gamma_{34}$ cross-sections from the $15_{\mathrm{N}}(\mathrm{p}, \gamma)$ reaction down to $\mathrm{E}_{\mathrm{p}}=4 \mathrm{MeV}$. These results are shown in Fig. 4.4.B-1. Below this energy the 8 -rays due to primary and secondary decays involving the $8.88 \mathrm{MeV}$ level fall in the same energy range as the $\gamma$-rays of interest and it becomes difficult to extract $\gamma_{12}$ and $\gamma_{34}$ yields. Below the neutron threshold the spectra become very clean, and une can identify both the primary and secondary $\gamma$-rays of the decays to the excited states of 160 . The evidence from the lower energy data $\left(2.6<\mathrm{E}_{\mathrm{p}}<4 \mathrm{MeV}\right)$ is that no sharp resonances exist in the $\gamma_{0}$ and $\gamma_{12}$ excitation functions with the exception of the region around $E_{p}=3 \mathrm{MeV}$ where pile-up from the $4.4 \mathrm{MeV} \gamma$-rays dominate the spectra and no conclusions can be made. The same procedure in extracting the cross-sections was followed for these data as for the data described in Section 4.4.A.

Our data overlap those of Barnett et a1. ${ }^{1}$ Although the agreement in the energy region above $6 \mathrm{MeV}$ is reasonable we observe very poor agreement in the lower energy data. The rise at lower energies of the Ref. 1 cross sections relative to ours demonstrates that the data of Ref. 1 suffer from strong background contributions below $6 \mathrm{MeV}$.

The most interesting feature of the excitation functions shown in Fig. 4.4. B-1 is the observed decay of the $1^{+}$states at 16.22 and $17.14 \mathrm{MeV}^{2}$ to the $6.050^{+}$first excited state of 160 . We have measured excitation functions at $\theta=90^{\circ}$ over these two resonances in $\sim 5 \mathrm{keV}$ steps (Fig. 4.4.8-2). We also measured angular distributions on and off the resonances to determine the resonant Legendre coefficients. The even Legendre coefficients for the resonance must be the same for the $\gamma_{0}$ and the $\gamma_{1}$ decay since the final states both have spin zero and hence the $\gamma_{1} / \gamma_{0}$ branching ratios may be determined from the $90^{\circ}$ data. Dur 12 resonance angular distributions are consistent with the $\gamma_{0}$ resonance angular distributions, but the errors are quite large $\left(a_{2} \sim 0.2 \pm\right.$ 0.3 for the 17.14 resonance and $-0.5 \pm 0.3$ for the $16.22 \mathrm{MeV}$ resonance). The resonance strength of the $\gamma_{12}$ decays rules out a significant contribution from $\gamma_{2}$, which would be $M 2$ or $E 3$. In extracting the resonance strengths we used the a 2 coefficient as determined from the $\gamma_{0}$ data. The present data combined with those of Ref. 2 result in improved values for these a 2 coefficients: $-0.761 \pm$ 0.061 and $0.30 \pm 0.10$ for the $16.22 \mathrm{MeV}$ and $1 / .14 \mathrm{MeV}$ resonances, respectively. The absolute normalization of these data was determined by matching the $\gamma_{0}$ yields at $7.33 \mathrm{MeV}$, taken at the same time, to the absolute cross-sections of Ref. 1. From the resonance areas of Fig. 4.4.B-2 we obtain $\Gamma \mathrm{p} / \Gamma \gamma_{\mathrm{o}} / \Gamma=2.77 \pm 0.34$ and $\Gamma_{\mathrm{p}} \Gamma_{\gamma_{1}} / \Gamma=0.34 \pm 0.05 \mathrm{keV}$ for the $16.22 \mathrm{MeV}$ state and $\Gamma_{\mathrm{p}} \Gamma \gamma_{\mathrm{O}} / \Gamma=4.12 \pm 0.58 \mathrm{eV}$ and $\Gamma_{\mathrm{p}} \Gamma \gamma_{1} / \Gamma=0.60 \pm 0.08 \mathrm{eV}$ for the $17.14 \mathrm{MeV}$ state. The ratio of radiative widths $I_{1} / \Gamma_{\gamma}$ is extracted more accurately from the ratio of the $90^{\circ}$ resonance cross-sections. For the $16.22 \mathrm{MeV}$ state we get 


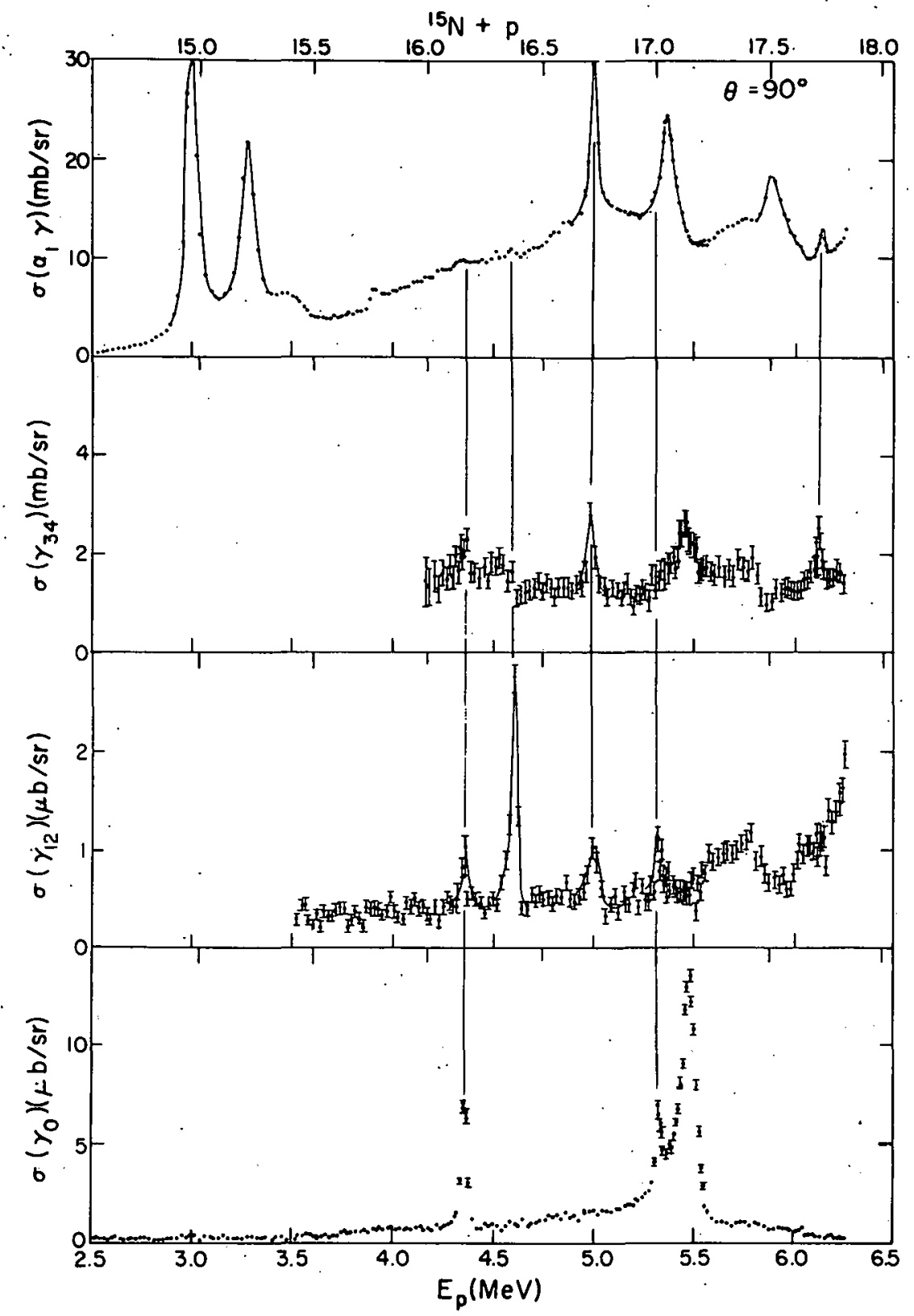

Fig. 4.4.B-1: Excitation function of the $\gamma$-rays resulting from the bombardment of ${ }^{15} \mathrm{~N}$ protons at energies below $6.2 \mathrm{MeV}$. Cross sections labeled $\sigma\left(\alpha_{1} \gamma\right)$ include contributions from the ${ }^{12} \mathrm{C}\left(\mathrm{p}, \mathrm{p}^{\prime} \gamma\right)$ reactions. 


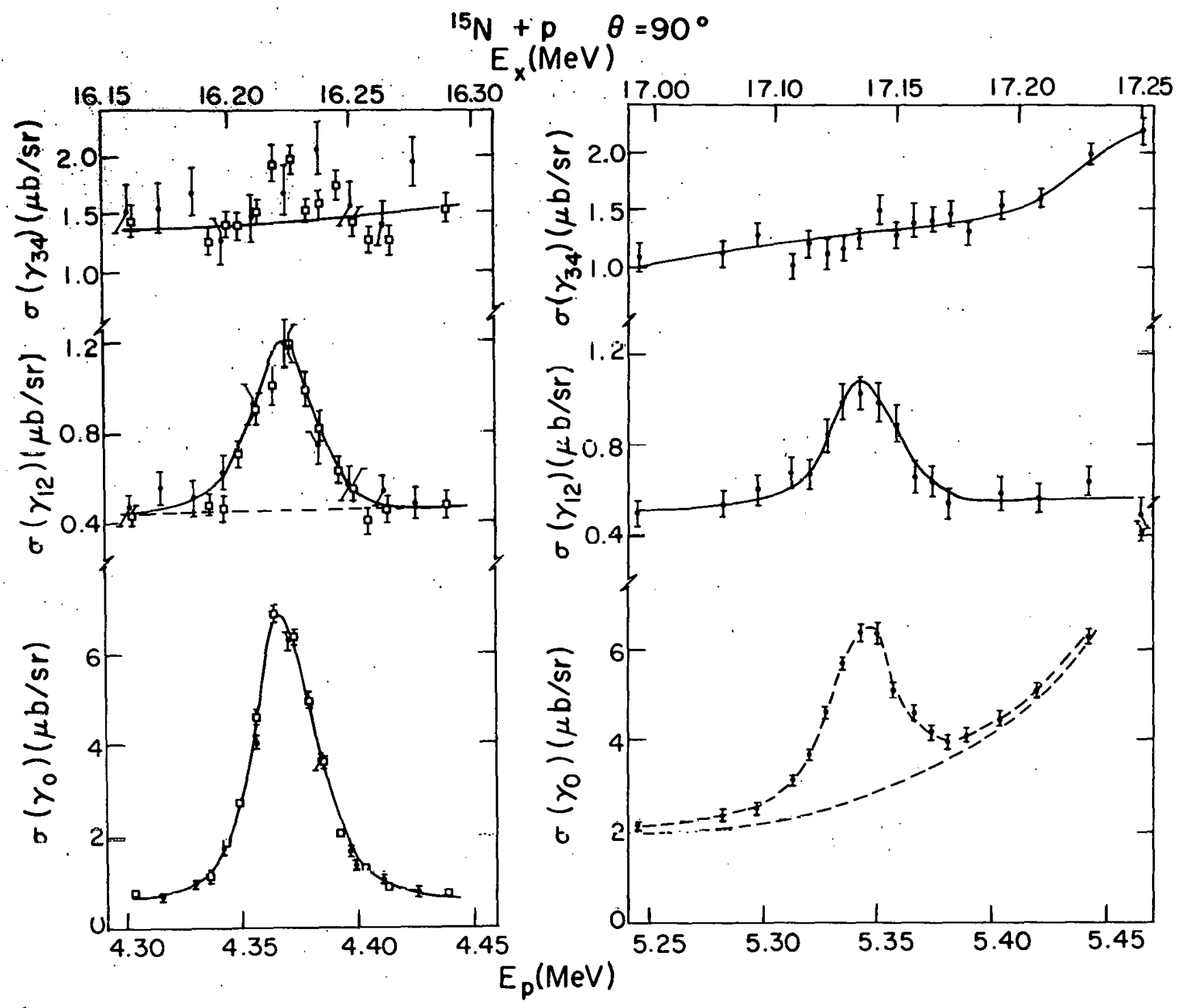

Fig. 4.4.B-2: Excitation functions in the vicinity of the 16.22 and 17.14 $\mathrm{MeV}$ states. 
$\Gamma \gamma_{1} / \Gamma_{\gamma_{O}}=0.110 \pm 0.007$ corresponding to a ratio of $\mathrm{B}\left(\mathrm{M1}, \gamma_{1}\right) / \mathrm{B}\left(\mathrm{M} 1, \gamma_{0}\right)=0.45 \pm$ 0.63 . The same ratios for the $17.14 \mathrm{MeV}$ state are $0.149 \pm 0.017$ and $0.55 \pm$ 0.04 , respectively. Thus we observe that the ratio of reduced strengths of the decay to the lst excited $0^{+}$state relative to the decay to the ground state is aproximately the same for the 16.22 and $17.14 \mathrm{MeV}$ initial states. It will be interesting to see if theoretical calculations can reproduce this result.

$\underline{\text { References }}$

1. A.R. Barnett, and M.W. Tanner, Nuc1. Phys. A152, 257 (1970).

2. K.A. Snover, P.G. Ikossi, and T.A. Trainor, Phys. Rev. Lett. 43, 117 (1979).

5. The ${ }^{54} \mathrm{Fe}\left(\alpha, \gamma_{0}\right)^{58} \mathrm{Ni}$ Reaction

K.J. Davis, P.G. Ikossi, and K.A. Snover

A. Introduction and Motivation

This experiment investigates the multipolarities of the gamma rays from the decay of the giant resonance region in ${ }^{58} \mathrm{Ni}$ to the ground state. This is accomplished by measuring the angular distribution of gamma rays produced in the reaction ${ }^{54} \mathrm{Fe}\left(\alpha, \gamma_{0}\right){ }^{58} \mathrm{Ni}$ for various al pha particle energies $(8.9-12.3 \mathrm{MeV})$ corresponding to the excitation of the giant resonance region. Our experiment is a repeat of one performed by Meyer-Schutzmeister et al. at Argonne National Laboratory. 1

Our experiment is motivated by an outstanding discrepancy between the E2 cross sections deduced by Meyer-Schutzmeister et al. ${ }^{1}$ for the ${ }^{54} \mathrm{Fe}\left(\gamma, \alpha_{0}\right){ }^{58} \mathrm{Ni}$ and the E2 strength observed in the ${ }^{58} \mathrm{Ni}\left(\alpha, \alpha^{-}, \alpha_{0}\right)^{54} \mathrm{Fe}$ reaction by Collins et a1.2 (of the University of Maryland). The Argonne group found that for excitation energies between 13.5 and $18.3 \mathrm{MeV} 3.9 \% 3$ of the EWSR is exhausted by this reaction. The Maryland investigation examines coincidences in the reaction ${ }^{58} \mathrm{Ni}\left(\alpha, \alpha^{-}, \alpha_{0}\right){ }^{54} \mathrm{Fe}$ from which they deduce the $\mathrm{E} 2$ cross section that would be observed in the ${ }^{58} \mathrm{Ni}\left(\gamma, \alpha_{0}\right){ }^{54} \mathrm{Fe}$ reaction. They find that in the giant resonance energy range the $E 2$ contribution in the $\alpha_{0}$ channe1 accounts for only $\sim 1 \%$ of the EWSR assuming both the resonance and the underlying continuum background decay in the $\alpha_{0}$ channel with the same probability. Furthermore, they set an upper limit of $3 \%$ of the EWSR for $\alpha_{0}$ decay of the giant E2 resonance by assuming no contribution from the background. In light of this discrepancy, we have undertaken a remeasurement of the ${ }^{54} \mathrm{Fe}\left(\alpha, \gamma_{0}\right){ }^{58} \mathrm{Ni}$ reaction.

B. Experimental Methods and Results

Our measurements were made using a target of $97 \%$ isotopically pure ${ }^{54} \mathrm{Fe}$. The target thickness was determined by measuring the energy loss of alpha particles from an ${ }^{251} \mathrm{Am}$ source passing through the target foil. The average 
thickness of the target over the beam spot was found to be $1.27 \pm 0.06 \mathrm{mg} / \mathrm{cm}^{2}$. The uncertainty in this measurement is dominated by the uncertainty in the stopping power data. 4

The target was bombarded with alpha particles at ten different energies between 8.9 and $12.3 \mathrm{MeV}$. At each energy gamma rays were detected using a $10^{\prime \prime} \mathrm{x}$ $10^{\prime \prime}$ NaI detector placed 18" from the target and at the angles $40^{\circ}, 60^{\circ}, 90^{\circ}$, $120^{\circ}$, and $140^{\circ}$ as measured from the beam axis.

The total detection efficiency of the NaI detector was measured with a thick carbon foil using the $15.1 \mathrm{MeV}$ gamma ray from an isolated resonance in the ${ }^{12} \mathrm{C}(\mathrm{p}, \mathrm{y}){ }^{13} \mathrm{~N}$ reaction. ${ }^{5}$ The product of the solid angle and detector efficiency was found to be $(2.58 \pm 0.13) \times 10^{-3} \times(4 \pi)$ ster.

A typical 8 -ray spectrum is shown in Fig. 4.5-1. The two peaks at the high energy end of the spectrum result from the decay of the giant resonance to the ground state $\left(\gamma_{n}\right)$ and to the first excited state. These two peaks overlap and it was necessary to use a peak fitting program (GFITV), to reliably extract the areas of the $\gamma_{0}$ peak. An empirical line shape based on the $15.11 \mathrm{MeV}$ gamma ray from the ${ }^{12} \mathrm{c}(\mathrm{p}, \gamma)$ reaction was used to fit these two gamma rays. The line drawn through the data is the best fit for two gamma rays with a fixed energy difference of $1.454 \mathrm{MeV}$, which is the difference in energy of the ground state and first excited state of $58 \mathrm{Ni}$.

A plot of the differential cross sections at $90^{\circ}$ versus incident alpha particle energy is shown in Fig. 4.5-2. The origin of the "discontinuity" between 10.7 and $11.0 \mathrm{MeV}$ is not known, but may be due to Erikson fluctuations or intermediate structure.

The angular distributions at each energy were analyzed to determine the relative strength of $E 1$ and E2 contributions to the total yield. The angular distributions were fit with a computer program assuming them to be due to only E1 and E2 amplitudes with an arbitrary relative phase angle. Finite angular attenuation coefficients were included in the analysis. The ratio of $\sigma(E 2)$ to $\sigma(E 1)$ was found to be less than 0.12 in all cases and the phase angle in general was near $90^{\circ}$ as typically found in other $\left(\alpha, \gamma_{0}\right)$ studies in this mass region. The angular distribution data points are shown with the fitted curves in Fig. 4.5-3. The error bars include only statistical errors.

The extracted $\left(\alpha, \gamma_{0}\right)$ E2 cross sections can be related to the cross section for the inverse reaction $58 \mathrm{Ni}\left(\gamma, \alpha_{0}\right)$ by detailed balance. The converted $\sigma_{E \cdot 2}\left(\gamma, \alpha_{0}\right)$ cross section is plotted versus gamma ray energy in Fig. 4.5-4. The dotted lines are an extrapolation of the data, based on the shape of the Argonne data, to cover the full energy range of the resonance. This graph may be used to calculate the E2 contribution to the EWSR from the $53_{\mathrm{Ni}}\left(\gamma, \alpha_{0}\right)$ channel. Our results indicate that only $(1.49 \pm 0.24) \%$ of the EWSR is exhausted over the energy range 14.5-17.7 MeV. The quoted uncertainty includes statistical errors as well as systematic errors related to target thickness, detector efficiency, and pile-up. 

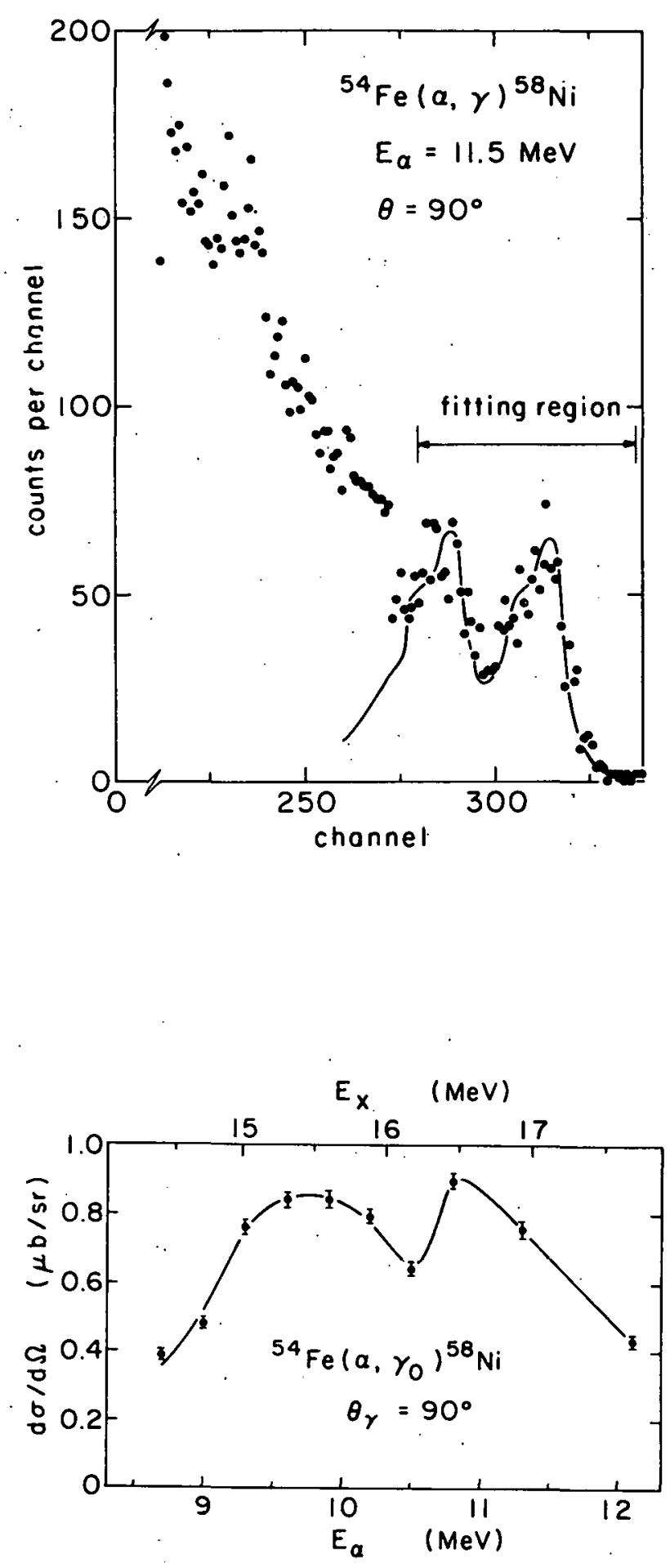

Fig. 4.5-1: The gamma ray spectrum for the reaction ${ }^{54} \mathrm{Fe}(\alpha, \gamma){ }^{58} \mathrm{Ni}$ at $\mathrm{E}_{\mathbf{X}}=11.5 \mathrm{MeV}$ and $\theta=90^{\circ}$ for $2000 \mu \mathrm{C}$ of collected charge. The solid line represents the fitted curve as described in the text.
Fig. 4.5-2: The differential crosssection at $90^{\circ}$. for the ${ }^{54} \mathrm{Fe}\left(\alpha, \gamma_{0}\right){ }^{58} \mathrm{Ni}$ reaction. The line is drawn to guide the eye. 

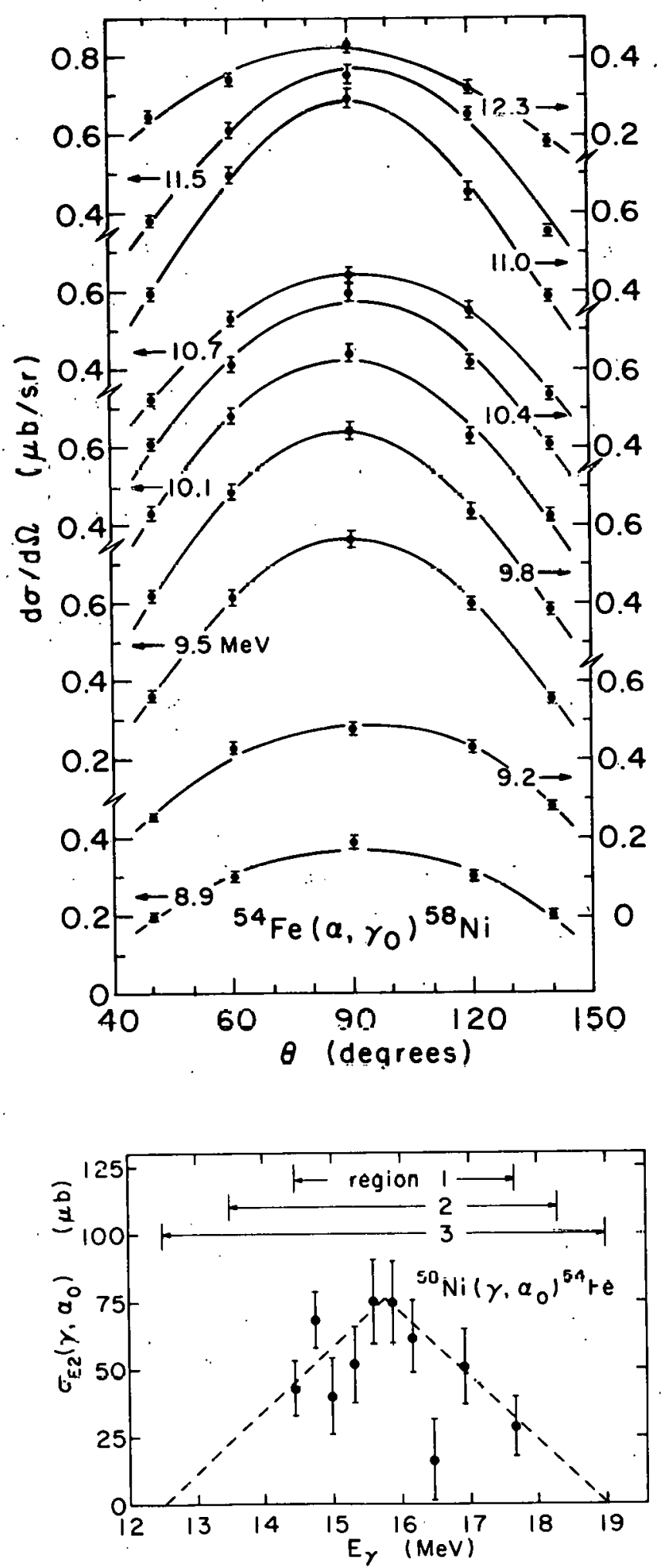

ilg. 4.5-3: Angular dislifibions for the ${ }^{54} \mathrm{Fe}\left(\alpha, Y_{0}\right){ }^{58} \mathrm{Ni}$ reaction taken at incident energies between 8.9 and 12.3 MeV. The error bars include only statistical errors. The lines are the least squares fitted curves as described in the text.

Fig. 4.5-4: The E2 cross-section for the ${ }^{58} \mathrm{Ni}\left(\gamma, \alpha_{0}\right){ }^{54} \mathrm{Fe}$ reaction as determincd from our data. The dotted lines are based on the shape of $\sigma_{E 2}$ deduced by MeyeiSchutzmeister et al. (Ref. 1) and are used to extrapolate our data tio larger energy ranges. The energy regions $1-3$ correspond to our experiment, the Argonne experiment (Ref. 1), and the Maryland experiment (Ref. 2), respectivcly. 


\section{Comparison with other Experịments}

A comparison of the differential cross section at $9.0^{\circ}$ indicates that the results of Meyer-Schutzmeister et al. are $\sim 30 \%$ higher than our values. In both experiments the resonances are centered at about the same energy, but the shapes of $\sigma\left(90^{\circ}\right)$ are somewhat different.

The average value of the ratio and error for $\sigma_{E 2} / \sigma_{E 1}$ determined from our data is $0.056 \pm 0.006$ compared with $0.10 \pm 0.05$ (over the same energy range) as determined from the data of Meyer-Schutzmeister et al. This ratio reflects only differences in angular distribution information and should be independent of any problems with absolute normalization. This difference may be due to improved peak area extraction in our analysis resulting from better gamma-ray energy resolution and lower background.

The dotted lines in Fig. 4.5-4 have been used to extrapolate our data to a wider energy range. This allows us to compare our results more directly with those of Meyer-Schutzmeister et al. and Collins et al. Table 4.5-1 shows the value of $\mathrm{S}_{\mathrm{E} 2}=\rho \mathrm{E} \sigma(E) \mathrm{dE} / \mathrm{E}^{2}$ of the three experiments in various energy ranges. We note that our value for the E2 contribution to the EWSR between 13.5 and 18.3 is $\sim 2 \%$ which is only half of the value found by the Argonne group. While this $2 \%$ result is somewhat larger than the $1 \%$ value determined by Collins et al. it is well below the $3 \%$ upper limit set by them.

\section{References}

1. L. Meyer-Schutzme1ster et al., Phys. Rev. 17C, 56 (1978).

2. M.J. Collins et a1.; Phys. Rev. Lett. 42, 1440 (1979).

3. Here we use $\left\langle\mathrm{r}^{2}\right\rangle 1+2=3.77 \pm 0.01$ in the $\overline{E W S R}$, as pointed out by G.J. Wagner in The Proceedings of the Giant Multipole Conference, Oak Ridge, Tennessee, Octoher 1979 (to be published).

4. J.F. Ziegler, Helium: Stopping Powers and Ranges in all Elemental Matter. Pergamon Press, New York, New York (1977).

5. R.E. Marrs, E.G. Adelberger, and K.A. Snover, Phys. Rev. C16, 61 (1977). See also R.E. Marrs, Ph.D. Dissertation (1975), University of Washington, unpub11shed.

Table 4.5-1. Table of percentages of EWSR for the ${ }^{58} \mathrm{Ni}\left(\gamma, \alpha_{0}\right)^{54}$ reaction over different energy ranges for various experiments

$\begin{array}{lccc} & \text { Region } 1 & \text { Region } 2 & \text { Region } 3 \\ & 14.5=17.1 & 13.5=18.3 & \underline{12: 5}=19.0 \\ \text { Washington } & 1.49 \%+0.24 \% & 1.98 \% & 2.2 \% \\ \text { Argonne } & - & 3.9 \% & 4.3 \% \\ \text { Maryland } & - & -1 \%\end{array}$




\section{MEDIUM ENERGY}

1. Continuum Structure Studies and Total Inelastic Cross Sections for Low Energy $\pi^{+}$Nucleus Scattering

K. Aniol, D. Chiang, I. Hal pern, and D. Storm ${ }^{+}$

Last year ${ }^{1}$ we reported result's of an angular distribution measurement of 67 $\mathrm{MeV} \pi^{+}$scattering on calcium. This year we have extended our results to cover more targets at more angles and we have taken data at other pion energies: 40, $50,60,75$, and especially at $85 \mathrm{MeV}$.

The emphasis last yoar wao on undessianding the location and strenglh of a bump in the $\pi$ spectrum of lighter elements that appears at the E2 giant resonance excitation energy. It was found that the angular distribution of the bump in Ni. and $\mathrm{Ca}$ (where it was most clearly seen) iequired a considerable admixture of giant dipole excitation. The description of these data and their analysis is given in the Ph.D. thesis of David Chiang. 2

The more recent runs have emphasized the gross features of the inelastic spectra, their angular distributions, their general shapes and the integrated cross-sections. It is hoped that such information at a number of energies and for a number of targets will allow us to deduce the relative probabilities for scattering events and meson absorptions in nuclear matter as a function of pion energy.

Our data taking rate was improved in last summer's run by the addition of a fast PID requirement in the hardware strobe in order to keep protons and electrons from clogging the data acquisition system. Several on-line tests confirmed that these hardware gates were not cutling 1nco the pion spectrum. A data-sorting and analysis routine was writton for our uewly acquired DEC-VAX culluter (see Sec. 10.12). It allows us to make various cuts and corrections that are required to obtain pure, clean pion spectra (see also Ref. 2).

Figure 5.1-1 shows total inelastic cross sections as a function of angle for a few targets. At both of the incident energies displayed in the figure ( 67 and $85 \mathrm{MeV}$ ) the observed spectra were integrated, for each point, from $\sim 23 \mathrm{MeV}$ (the detector cutoff) to an energy $6 \mathrm{MeV}$ below the elastic line. It was not possible, hecause of muon background, to measure forward of $40^{\circ}$. It may be true nevertheless, that we have not missed much of the total inelastic cross section since the angular distributions are so otrongly backward peaked. There are a number of factors. that contribute to the backward peaking. First, the free pion-nucleon cross section at these energies is backward peaked. Further, because the pion is light one does not get the large forward folding that one gets when transforming p-nucleon collisions in a nucleus into the laboratory frame. Finally the absorption of pions tends to make it less likely for forward than for backward scattered pions to escape following nuclear collisions with impact parameters appreciably smaller than the radius. 

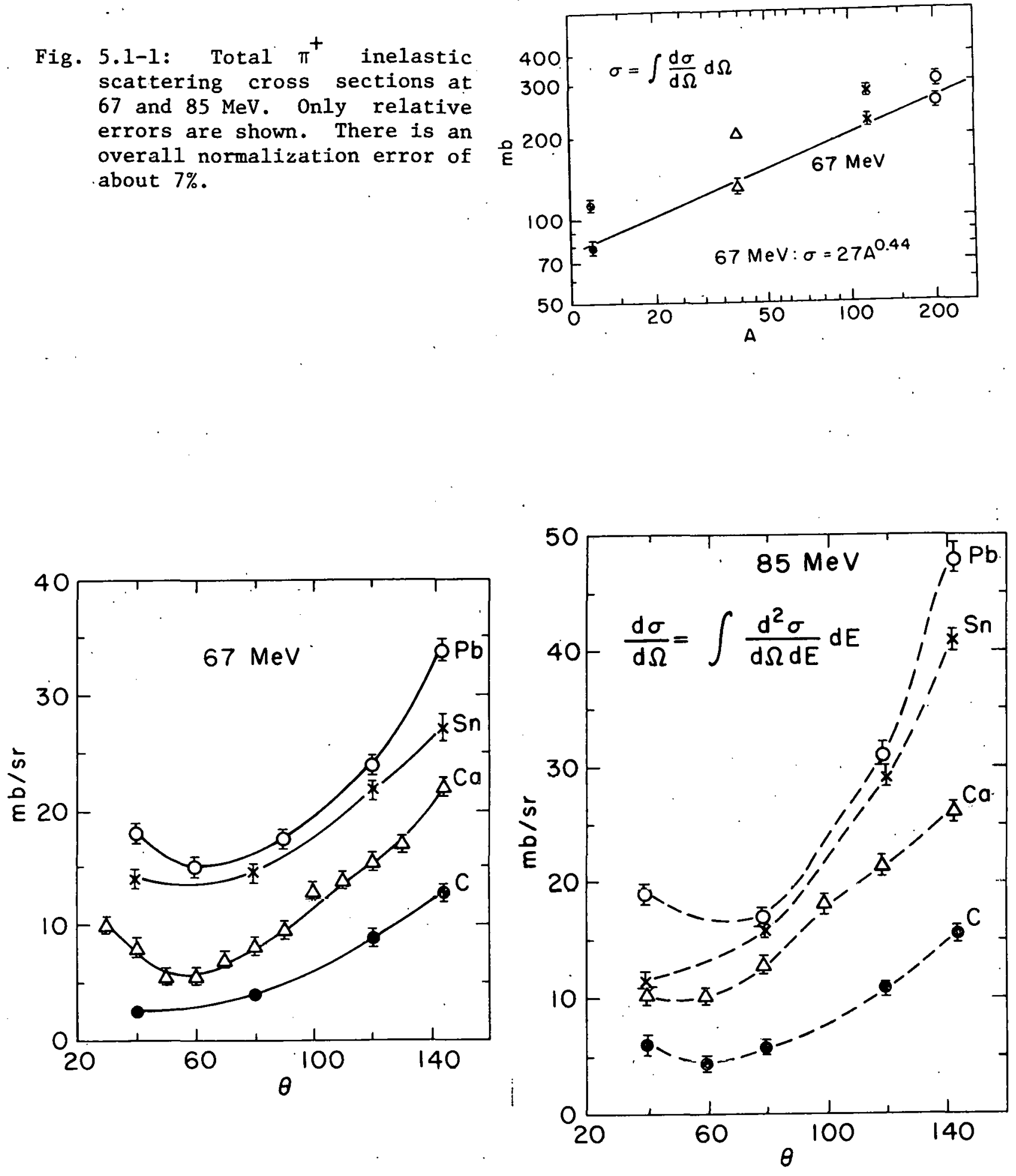

Fig. 5.1-2: Total differential cross section for inelastic scattering for 67 and $85 \mathrm{MeV} \pi^{+}$ 
We are currently making estimates of expected cross-scctions and angular distributions on the basis of nodels which consider all of the forementioned factors as well as the effects of the nucleon momentum distribution and of Pauli blocking. Some of the observable features of the cross-sections which we would like to explain with a consistent model include the more conspicuous leveling off or saturation of cross-sections with large A at $85 \mathrm{MeV}$ than at $67 \mathrm{MeV}$ (Fig. 5.1-2) and the apparent backward drift of the minimum in the angular distributions with increasing A.

In a preliminary analysis of the $67 \mathrm{MeV}$ data, ${ }^{2}$ we find that the A dependence of the total $\pi^{+}$inelastic cross-sections can be accounted for if, on the average, it is twice as likely for a pion to be absorbed in interactions that remove it from the entrance channel on its way through the nucleus as it is for the pion to be simply scattered. This analysis is based on a comparison of the energy and angle integraced inelastic crose-section and the total reaction cross-section.

Figure 5.1-3a shows some typical spectra in a heavier target. It is interesting to note that at forward angles the spectra are rather flat with energy whereas at backward angles, the spectrum has a positive slope as a function of outgoing pion energy. This is not how the inelastic spectra of protons, $\alpha$ particles and other well-studied strongly interacting projectiles behave. They have a negative slope. A good part of this negative slope is generally attributed to the effects of multiple scattering. In ( $p, p^{\prime}$ ) and $\left(\alpha, \alpha^{\prime}\right)$ one expects a larger ratio of multiple to single scattering events at backward than at forward angles and multiply-scattered projectiles tend to emerge with less energy than those that are gingly ecattered. This raises the possibility that the opposite spectral shape differences, fore and aft, which one observes for the pions are also due in part to multiple scattering. Because

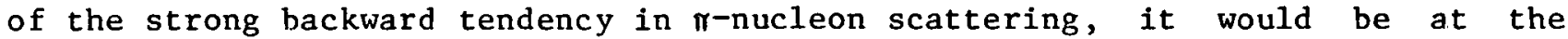
forward angles that the multiple/single ratio would be higher than average and where one would therefore expect relatively more scatterings with large energy loss.

A curious feature of the backward spectra is their striking similarity in shape and magnitude (F1gure $5.1-3 b$ ) at 67 and $85 \mathrm{MeV}$ when $\mathrm{d}^{2} \sigma / \mathrm{dO} \mathrm{dE}$ is expressed in terms of outgoing pion energy.

\section{References}

+ Our collaborators on this experiment are the Carnegie-Mellon University Group headed by Profs. P. Barnes, R. Eisenstein, and W. Wharton. We are collaborating with Prof. G. Miller of uue department on the interpretation of the results.

1. Nuclear Physics Laboratory, University of Washington Annual Report (1979).

2. David Chiang, Ph.D. Thesis, University of Washington, 1980 . 
Fig. 5.1-3a: Inelastic $\pi^{+}$scattering on $\mathrm{Sn}$ at $85 \mathrm{MeV}$. The specified shapes observed here are typical of those observed from $\mathrm{Ca}$ to $\mathrm{Pb}$.
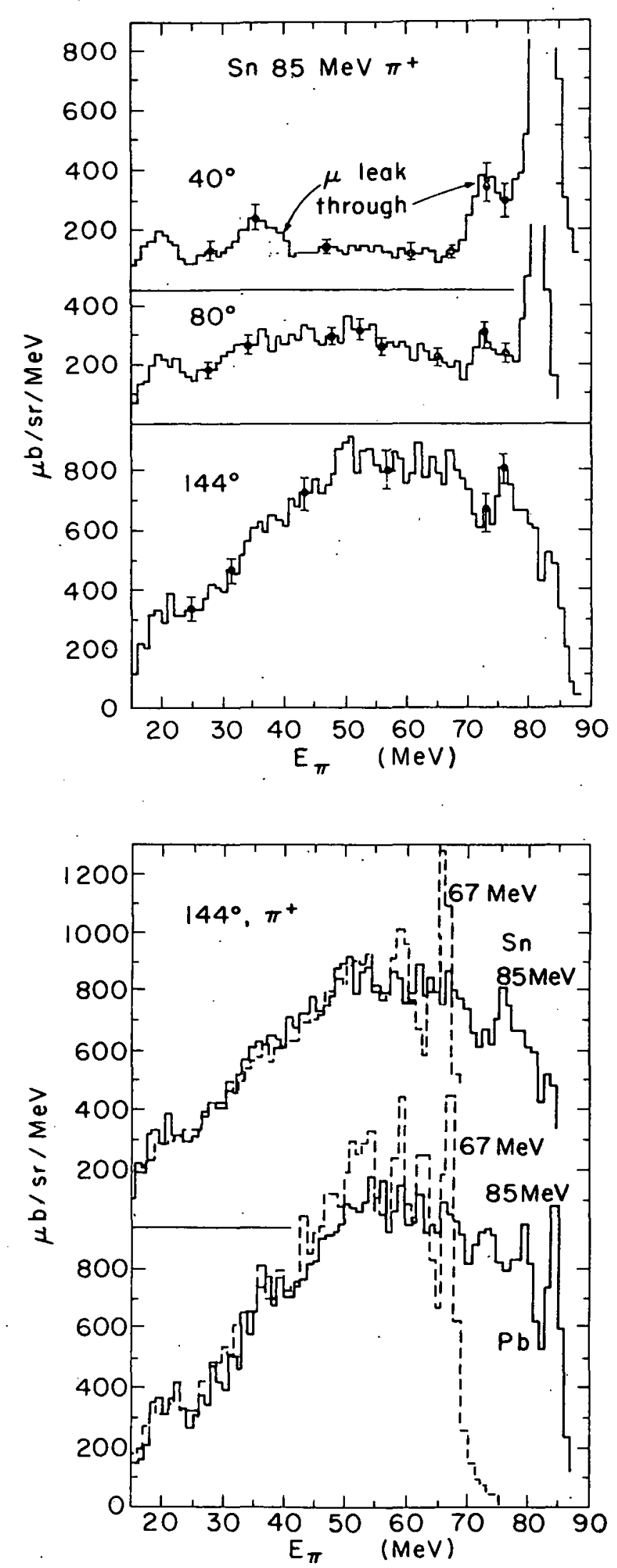

Fig. 5.1-3b: Comparison of from $\mathrm{Sn}$ and $\mathrm{Pb}$ at 67 (dashed) and 85 (solid)) MeV. For large scattering angle the spectra at the two energies match well with each other as a function of outgoing $\pi$ energy. 
2. Photoneutron Multiplicities at Medium Energies

I. Hal pern

It is only recently that reasonable values for photon absorption cross-sections at medium energies have become available. The reason that it has been difficult to measure so fundamental a quantity is that the machines which produced high energy photons, generally made them with continuous (bremsstrahlung) spectra. This feature requires subtractive techniques to determine cross-sections at a given energy. These techniques are unfortunately limited by the fact that medium energy photon cross-sections are significantly smaller than those in the lower-lying giant resonance region.

With the advent of monochromator techniques, it has become possible to measure some cross-sections up to $140 \mathrm{MeV}$ with substantial prec1sion. 1 This work is leading to a revival of interest in the processes by which medium energy photons are absorbed, a fleld that has been somewhat dormant for lack of useful experimental material.

The recent measurements at Saclay ${ }^{1}$ identify absorptions as events where one or more neutrons are detected from a nuclear interaction. One makes the very reasonable assumption that, for the heavier nuclei for which the method applies, at least one neutron is emitted for each high energy photon absorbed. In the course of counting the events where one or more neutrons are emitted to determine an absorption cross-section, one obtains a neutron multiplicity distribution. We have been looking into the problem of extracting information about the absorption process from the measurements of these measured multiplicity distributions.

. This is a brief status report on this problem. The most reliable feature of the measured neutron multiplicity distribution at any energy is its mean value, $\overline{n_{m}}$, where the subscript stands for "measured." The true mean multiplicity, $\overline{n_{t}}$ is $1 / \varepsilon \overline{n_{m}}$ where $\varepsilon$ is the mean neutron detection efficiency, typically $60 \%$ to $70 \%$ for the $4 \pi$ geometry used at Saclay. The maximum possible multiplicity at any energy is given by the maximum number of neutrons that can be evaporated from the target when it absorbs the photon. The ratio of the observed mean multiplicity to this maximum multiplicity can be used to deduce the fraction of the input energy which is carried off by fast particles in the initial direct interaction. For example for $\mathrm{Pb}$ at $\mathrm{E}=70 \mathrm{MeV}$ one finds that about $45 \%$ of the energy is carried off by fast nucleons. This number, deduced from the measured multiplicity, is in excellent accord with the fast neutron spectrum and the cross-section at this energy measured many years ago by a difference method. 2 The energy dependence of this mean multiplicity tells us about the energy dependence of the energy loss through fast nucleon emission, following photoabsorption. The interpretation of this energy dependence will depend on the nature of the primary interaction, e.g., on how many nucleons are involved. It will also depend on secondary interactions which determine the chances for escape of the fast nucleons from the nucleus. I'here is no firm theoretical model for the primary interaction and the relevant nucleon mean free paths at medium energies can only be roughly estimated at this time. 
The most popular description of the absorption of photons at medium energies is the so-called quasi-deuteron model.3 It suggests a smooth energy dependence for the absorption cross-section and is often characterized by two parameters, a damping parameter which controls the shape of the cross-section curve and an "effective number of quasi-deuterons" which controls its magnitude. Neither parameter is known with useful precision from theoretical considerations.

To use the data to evaluate these or other parameters referring to the photon absorption process, one wants to handle, in a common framework, many features of the observations--e.g., the energy and target mass dependence of $\overline{n_{t}}$.

The multiplicity data can also provide useful values for the second moment of the distribution, namely

$$
\overline{n_{t}^{2}}=\frac{1}{\varepsilon^{2}}\left(\overline{n_{m}^{2}}-(1-\varepsilon) \overline{n_{m}}\right)
$$

but it is unlikely that, with present techniques, still higher moments can be measured with adequate certainty. This second moment is very interesting since it provides a measure of the width of the multiplicity distribution. A comparison of this width with those of measured fast photoneutron and photoproton spectra can be used to deduce information about correlations between these emissions. That is, (subject to corrections for intranuclear cascading) one can learn something of the energy sharing between emitted fast particles, and in turn this can tell us about basic features of the primary absorption process. As in other medium energy reactions, final state interactions tend to obscure the features of primary interactions. Only by treating many nuclei at many energies from various viewpoints can one hope to disentangle secondary matters, like mean free paths for escape, from primary concerns.. We are hopeful that the measurement of the moments of photonentron multiplicity distributions as a function of $E$ and $A$ will provide sufficiently important constraints to help us" sharpen our picture of the interaction of medium energy photons with nuclei.

\section{References}

$+\quad$ This problem was brought to our attention last year when I. Hal pern was on sabbatical leave at Saclay. He is indebted to $R$. Bergere and A. Leprêtre for discussions of their preliminary data.

1. A. Veyssiere et al., N.I.M., 165, 417 (1979), and A. Lepretre et al., Phys. Lett. $\overline{79}, 43$ (1978).

2. N.N. Kaushal et a.1., Phys. Rev. 175, 1330 (1968).

3. J.Levinger, Phys. Lett. 82B, $181 \overline{(1979) ; ~ P h y s . ~ R e v . ~ 84, ~} 43$ (1951). 


\section{HEAVY ION REACTIONS}

\section{Angular Momentum Dependent Level Density Limitations to Fusion}

\section{A.J. Lazzarini and R. Vandenbosch}

Recent results on the fusion cross sections for several pairs of reactions leading to the same compound nuclei indicate that at bombarding energies somewhat above the Coulomb barrier the fusion cross section may be determined by properties of the compound nucleus. If all $\ell$ values up to a sharp cutoff $\ell_{c}$ are assumed to be responsible for the fusion cross section, the $l_{c}$ values deduced from the experimental cross sections for different entrance channels leading to the same compound nucleus are the same for a given excitation energy. This behavior for the $160+16_{0}$ and ${ }^{12} \mathrm{C}+{ }^{20} \mathrm{Ne}$ entrance channels to the ${ }^{32} \mathrm{~S}$ compound system is illustrated in Fig, $6.1-1$. The horizontal bars $1-2$ and open circles represent $l_{c}$ values for the $16_{0}+16_{0}$ entrance channel, while the triangles ${ }^{4}$ are for the ${ }^{12} \mathrm{C}+{ }^{20} \mathrm{Ne}$ system. A similar behavior is exhibited by the ${ }^{26} \mathrm{Al}$ compound system as shown in Fig. 6.1-2. The open circles 5,6 represent $l$ values for the ${ }^{12} \mathrm{C}+{ }^{14} \mathrm{~N}$ entrance channel, while the crosses are for the $10_{\mathrm{B}}+16_{0}$ system.

We have previously suggested ${ }^{7}$ that a plausible necessary condition for fusion to occur is that the compound nucleus have overlapping levels for all angular momenta which can lead to fusion. It has been shown that this criterion can account in a natural way for both the absolute magnitude and gross structure of the fusion excitation function of the ${ }^{12} \mathrm{C}+{ }^{12} \mathrm{C}$ system. This same criterion, expressed in sharp-cutoff form by $\Gamma / D_{J}=1$, accounts nicely for the $l_{c}$ values observed for forming the ${ }^{26} \mathrm{Al}$ and $32 \mathrm{~s}$ compound nuclei. The full curves: in the figures give the $J_{c}$ values which $D_{J}=\Gamma$, assuming a Fermi gas level density expression and taking $\Gamma$ from fluctuation studies. The level density parameters, $\dot{a}$ and $\Delta$, of Gilbert and Cameron ${ }^{8}$ were used, and the moment of inertia was calculated for a rigld body with $r_{0}=1.16 \mathrm{fm}$. The resulting $\mathrm{J}_{c}$ values account quite well for the critical angular momenta at intermediate energies, especially considering the uncertainties in the level density parameters. At low energies the entrance channel limits the fusion cross sections, as shown in Fig. $6.1-1$ by the $l_{g}$ values from optical model calculations. At the very highest bombarding energies the fusion cross sections may be limited by the maximum angular momentum for which the rotating liquid drop model predicts a finite fission barrier. This limit is included by the vertical line in Fig. 6.1-2.

It appears from the moct recent calrnlations that the yrast line itself cannot be playing a role in limiting fusion cross sections. The lower full curve in Fig. $6.1^{-1}$ shows the results of a calculation by Diebel et al. 9

\section{References}

1. D.C. Kovar et a1., Phys. Rev. C20, 1305 (1979).

2. I. Tserruya et al., Phys. Rev. $\underline{\mathrm{C} 18}, 1688$ (1978). 
3... B. Fernandez et al., Nucl. Phys. A306, 259 (1978).

4. F. Saint Laurent et a1., Nucl. Phys. A327, 5.17 (1979).

5. J. Gomez del Campo et al., Phys. Rev. Lett. 43, 26 (1979).

6. M. Con jeaud, S. Gary, S. Harar, and J.P. Wieleczko, Nucl. Phys. A209, 515 (1978).

7. R. Vandenbosch, Phys. Lett. 87B, 183 (1979).

8. A. Gilbert and A.G.W: Cameron, Can. J. Phys. 43, 1446 (1965).

9. M. Diebel, D. Glas, U. Mose1, and H. Chandra, Nuc1. Phys. (1979).

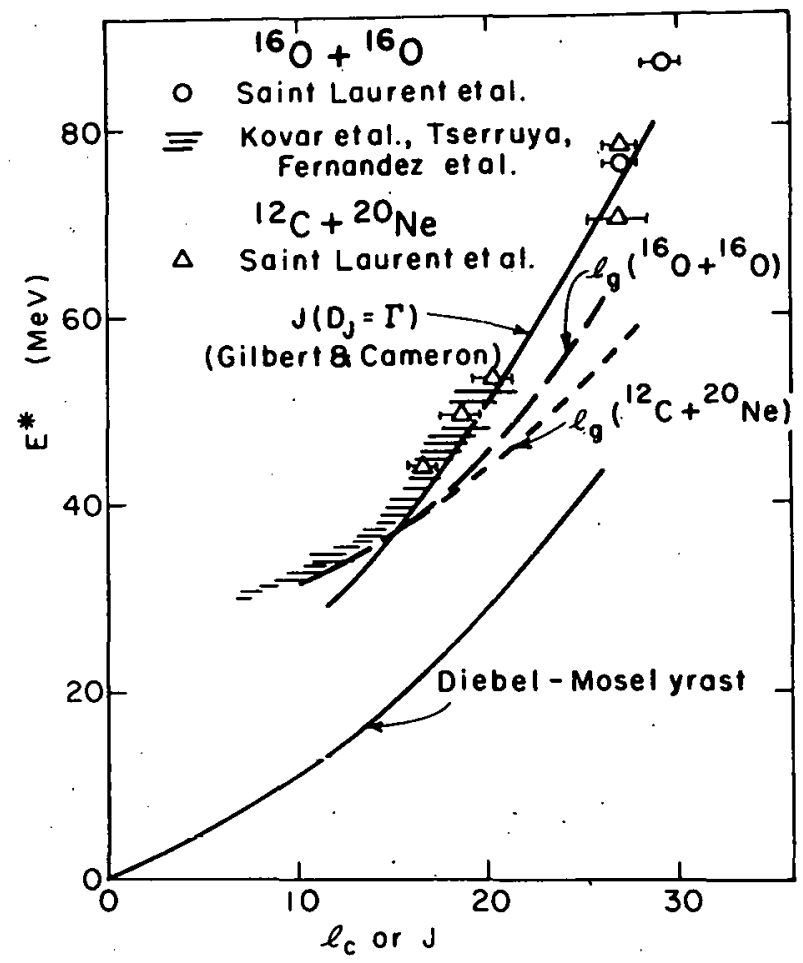

Fig. 6.1-1: Critical angular momenta derived from experimental fusion cross sections are indicated by the open symbols and horizontal bars. The upper full curve shows the limiting angular momenta derived from the criterion $\Gamma / D_{J}=1$.

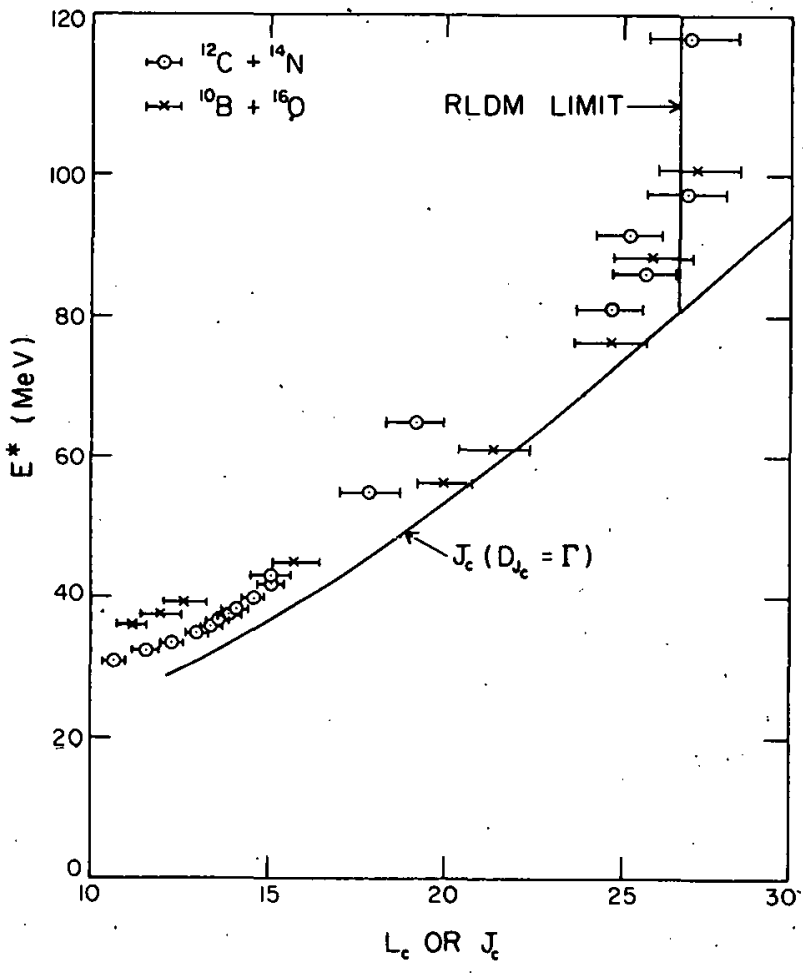

Fig. 6.1-2: Critical angular momenta derived from experimental fusion cross sections are compared with the limiting angular momenta derived. from the criterion $\Gamma / \mathrm{D}_{\mathrm{J}}=1$. 
2. Search for $\gamma$-rays from the Quasi-molecular ${ }^{12} c+{ }^{12} \mathrm{C}$ system

A. Lazzarini, K. Lesko, V. Metag, K. Snover, and R. Vandenbosch

Pronounced structures in the excitation functions for the ${ }^{12} \mathrm{C}+{ }^{12} \mathrm{C}$ elastic and inelastic scattering and various reaction channels have been interpreted using two opposing hypotheses. In the one case the observed structures are explained in a simple direct reaction model ${ }^{1}$ as arising from the dominance of certain partial waves, in the other case these structures are related to the existence of quasi-molecular excitations ${ }^{2}, 3$ corresponding to strongly deformed rotational states in $24 \mathrm{Mg}$.

A crucial test for the latter hypothesis would be the observation of collective $\gamma$-transitions between two quasi-bound states of the 24 nucleon system. Even if the $\gamma$-transitions are strongly enhanced $(\approx 150 \mathrm{Wu}$ ) as expected for very deformed nuclear statco the branching ratio for gamma to particle decay of the resonances is estimated to be only of the order of $10^{-5}$. Cousequently, the observation of these rare events of interest requires an experimental arrangement with an optimized detection efficiency which allows a kinematically complete and redundant identification of all reaction products in order to eliminate various sources of background.

The charge and energy of the reaction products, as well as their position in $\theta$ and $\phi$, are measured in coincidence with two position sensitive gas $\triangle E$, solid state E telescopes (see Sec. 9.1) subtending angles in the laboratory system between $30^{\circ}$ and $50^{\circ}$. Coincident $\gamma$-rays are detected in a 10" $x$ 10" NaI-crystal positioned $10 \mathrm{~cm}$ away from the target. A 3-fold coincident event is characterized by 11 parameters. Singles, 2-fold, and 3-fold coincidences have bccn ftored on magnetic tape event by event using the on-line data collection system described in Sec. 10.4. In the off-linc analysis, in addition to the usual time and $\triangle E / E$. constraints, all events have been checked for coplanarity and momentum conservation utilizing the position and energy information provided by the particle detectors.

Tn three separate experiments a total of $1.5 \times 10^{7}$ carbon-carbon 2-fold coincidences and $1.05 \times 10^{6} \mathrm{C}+\mathrm{C}+\gamma$ triple coincidences have been accumulated. The distribution of the $\mathrm{C}+\mathrm{C}+\gamma$ coincidences is shown in Fig. $6.2-1$ in a scatter plot of events as a function of $\gamma$-ray energy and the summed energy of the two carbon ions, which is directly related to the reaction Q-value. Two groups of events with $Q=-4.43 \mathrm{MeV}$ and $Q=-8.86 \mathrm{MeV}$ are apparent, corresponding to the excitation of one or both $12 \mathrm{C}$ jons to the $2^{+}$state at 4.43 $\mathrm{MeV}$ in the decay of the state at $\mathrm{E}_{\mathrm{cm}}=25.2 \mathrm{MeV}$. Due to Compton scattering and pair production the reglsteied $\gamma$-ray onergies are spread over a large energy range. The strons intcneity at $4.43 \mathrm{MeV}$ for $Q=-8.86 \mathrm{MeV}$ correspuids to the detection of only one of the two emitted $4.43 \mathrm{MeV} \gamma$-raye. Eventa for which the observed energy in particle and $\gamma$-ray detectors is within the particle energy and photopeak resolution of (including Doppler shift and single escape peak) 300 $\mathrm{keV}$ and $900 \mathrm{keV}$, respectively, will fall between the two straight lines shown in Fig. 6.2-1. Events beyond this 1 imit are attributed to pile up or chance coincidences. In the Q-value range between -5.7 and $-7.3 \mathrm{MeV} 16$ evcnts are observed which fulfill the total energy constraint. 


\section{PAGES 109 to 110 WERE INTENTIONALLY LEFT BLANK}




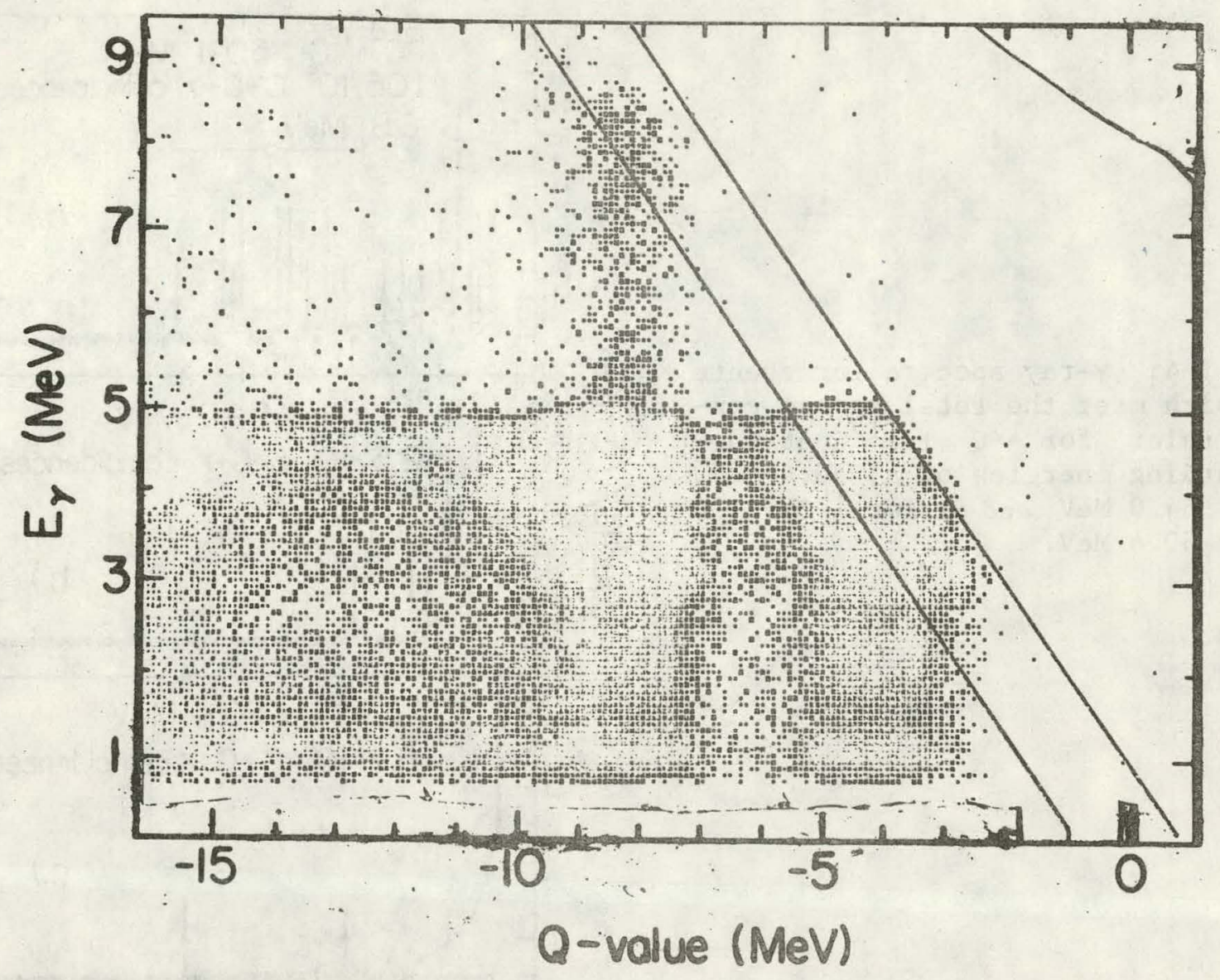

Fig. 6.2-3: Scatter plot of $\gamma$-ray energy versus the reaction Q-value for ${ }^{12} \mathrm{C}+{ }^{13} \mathrm{C}$ at a bombaring energy of $50.4 \mathrm{Mev}$. Small squares correspond to 1 event, big squares to $>2$ events. The two straight lines limit the region of events for which the total energy observed in the $\gamma$-ray and particle detectors agree within the resolution with the bombarding energy.

Assuming that $\Gamma_{c} / \Gamma_{\text {tot }}$ is the same for the $12^{+}$and $14^{+}$states the ratio of the 16 events of interest to the total number of $\mathrm{C}+\mathrm{C}+\gamma$ coincidences would imply a branching ratio of $(\mathrm{T} / \mathrm{\Gamma}$ tot $){ }_{1} 4^{+} \sim 1.5 \times 10^{-5}$. With a total width of $\Gamma$ tot $\sim 500 \mathrm{kev}$ the observed $\gamma$-ray intensity corresponds to a $B(E 2)$ value of 160 spu from which a quadrupole moment of $Q=1.4 \mathrm{~b}$ is deduced within the rotational model for the charge distribution of the 24-nucleon system. In a Strutinsky type calculation of the potential energy surface using a two-center shell model Chandra and Mose $1^{4}$ predict a quadrupole moment of $1.8 \mathrm{~b}$ for the molecular configuration of two touching spheroidal ${ }^{12} \mathrm{C}$ nuclei.

In view of the background of unknown origin mentioned above, however, we consider the present experimental result rather an upper limit on the $\gamma$-ray transition probability between the postulated $14^{+}$and $12^{+}$quasi-molecular rotational states. 
Fig. 6.1-4: $\gamma$-ray spectra for events which meet the total energy constraint for ${ }^{12} \mathrm{C}+{ }^{12} \mathrm{C}$ at bombarding energies of a) $50.4 \mathrm{MeV}$. b) $54.11 \mathrm{MeV}$, and c) for ${ }^{12} \mathrm{C}+13 \mathrm{C}$ at $50.4 \mathrm{MeV}$.

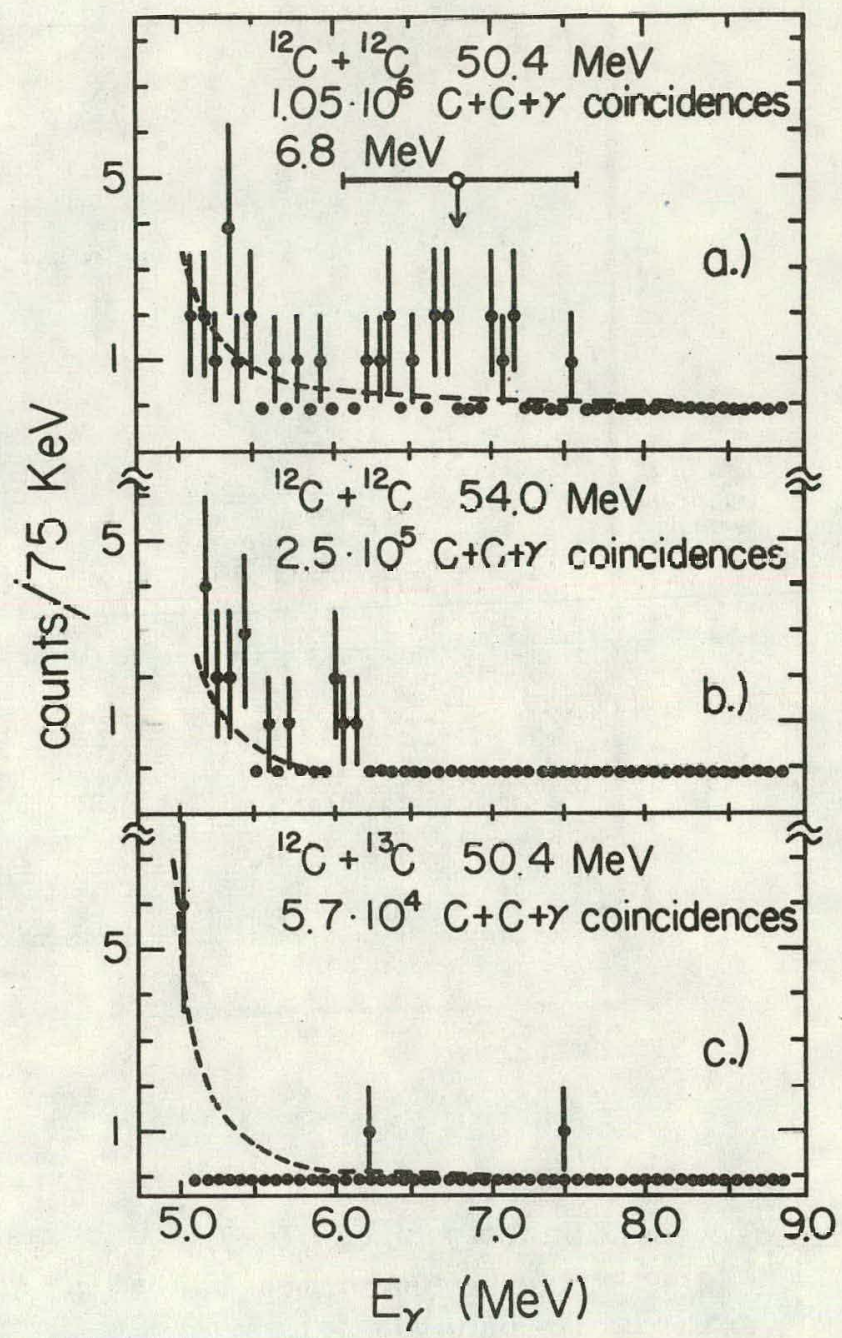

References

1. L.E. Cannell, R.W. Zurmuhle, and D.P. Balamuth, Phys. Rev. Lett. 43, 837 (1979).

2. E.R. Cosman, T.M. Cormier, K. van Bibber, A. Sperduto, G. Young, J. Erskine, L.R. Greenwood, and 0. Hansen, Phys. Rev. Lett. 35, 265 (1975).

3. T.M. Cormier, C.M. Jachcinski, G.M. Berkowitz, P. Braun-Munzinger, P.M. Cormier, M. Ga, J.W. Harris, J. Barrette, and H.E. Wegner, Phys. Rev. Lett. 40,924 (1978).

4. H. Chandra and U. Mosel, Nucl. Phys. A298, 151 (1978). 
H. Doubre,* A. Lazzarini, K.T. Lesko, D. Leach, A. Seamster, and

R. Vandenbosch

A measurement for the fusion cross section of the ${ }^{12} \mathrm{C}+{ }^{28} \mathrm{Si}$ system is in progress for center-of-mass energies between 18.4 and $28.2 \mathrm{MeV}$. This measurement was motivated by recent observation of structure in the elastic and inelastic back angle cross sections for this system.1,2,3 Presently the heaviest system exhibiting convincing evidence of structure in the fusion excitation function is the $16_{0}+16_{0}$ system.

A carbon target was bombarded with a ${ }^{28} \mathrm{Si}$ beam. The evaporation residues were observed in a position sensitive gas $\triangle E$-solid state $E$ telescope. The gas proportional counter $\Delta \mathrm{E}$ detector was masked with four vertical slits, each subtending $\sim 0.32$ degrees and separated from the next by $\sim 0.85$ degrees in the lab. The detector telescope was mounted on a movable arm in the 60 inch scattering chamber. Masking the entrance of the gas counter allowed the simultaneous measurement of four separate angles.

The data were recorded on magnetic tape in an event by event mode of the SDS 930 computer, and later analyzed off-1ine on the PDP $11 / 60$ computer. The data were sorted to separate the four angles and the resulting $\triangle E-E$ scatter plots were gated to extract the elastic and fusion yields for each angle and beam energy.

We have recently obtained a Monte Carlo code which will generate both the differential and total fusion cross sections for this system. 4 We are in the process of fitting our data to these predictions in order to obtain the excitation function. The preliminary results are indicated in Table 6.3-1 below. A more complete analysis of the data is in progress.

\section{References}

* H. Doubre visiting here from Institut de Physique Nucleaire, ORSAY, France.

1. P. Braun-Munzinger, et a1., Phyo. Rev. Lett. 38, 944 (1978).

2. J. Barrette, et al., Phys. Rev. Lett. 40, 445 (1978).

3. T. Reaner et al., Phys. Rev. Lett. 18, 1927 (1978).

4. Monte Carlo code, LPACE, written by A. Gavron at Oak Ridge National National Laboratory, Oak Ridge, Tenn. 
Table 4.3-1: ${ }^{28} \mathrm{Si}+{ }^{12} \mathrm{C}$ excitation function

$\begin{array}{cr}\text { E.C.M. (MeV) } & \text { Cross Section (mb) } \\ 18.42 & 612(23) \\ 21.21 & 930(46) \\ 22.62 & 886(43) \\ 23.07 & 1020(41) \\ 23.52 & 870(35) \\ 24.36 & 1144(44) \\ 25.20 & 1034(43) \\ 26.34 & 1070(43) \\ 28.20 & 1100(43)\end{array}$

4. Ecarch ful Instantaneous Fission in the ${ }^{208} \mathrm{~Pb}+{ }^{238} \mathrm{U}$ System

H. Doubre, A. Lazzarini, V. Metag, E.B. Norman, R.J. Puigh, A.G.

Seamster, and R. Vandenbosch

Experimental studies of heavy ion induced fission have so far provided evidence consistent with a sequential fission process.1,2 For small impact parameters, however, Deubler and Dietrich3 predict an instantaneous fission process in which fission is supposed to occur during the collision time so that angular distributions and kinetic energies of the emerging fission fragments are strongly affected by the Coulomb and nuclear forces exerted by the projectile. The experimental signature of this reaction mechanism is that--in contrast to sequential fission--the heavy fission fragment is always emitted in the forward direction close to the beam.

We have studied the $\mathrm{Pb}+\mathrm{U}$ system, for which a detalled calculation ${ }^{3}$ has been performed, at the SuperHILAC of the Lawrence Berkeley Laboratory. The experimental arrangement is shown in Fig. 6.4-1.

Fission fragments close to the beam direction are registered in an annular Si-surface barrier detector subtending $3^{\circ}-9^{\circ}$ in the laboratory system. A 45 $\mathrm{mg} / \mathrm{cm}^{2}$ thick $\mathrm{Ta}$ foil in front of the detector stops elastically scattered $\mathrm{Pb}$ ions at a bombarding energy of $1400 \mathrm{MeV}$ whereas it can be penetrated by heavy fission fragments ( $\mathrm{e} \cdot \mathrm{g} \cdot, 140 \mathrm{Xe}$ ) with energies $>1000 \mathrm{MeV}$ and 1 ight fission fragments (e.g., $98 \mathrm{Sr}$ ) with energies $>700 \mathrm{MeV}$. The charge and energy of the second flseion fragment, as well as 1 ts position in both $\theta$ and $\phi$, are measured with a position sensitive gas $\triangle \mathrm{E}$, solid state $\mathrm{E}$ telescope subtending angles in the laboratory between $200_{-40}^{\circ}$ in the reaction plane. Recoiling projectiles are recorded in a position sensitive solid state detector subtending angles of $360-84^{\circ}$ in the laboratory, corresponding to $70^{\circ}-160^{\circ}$ in the $\mathrm{CM}$ system. 

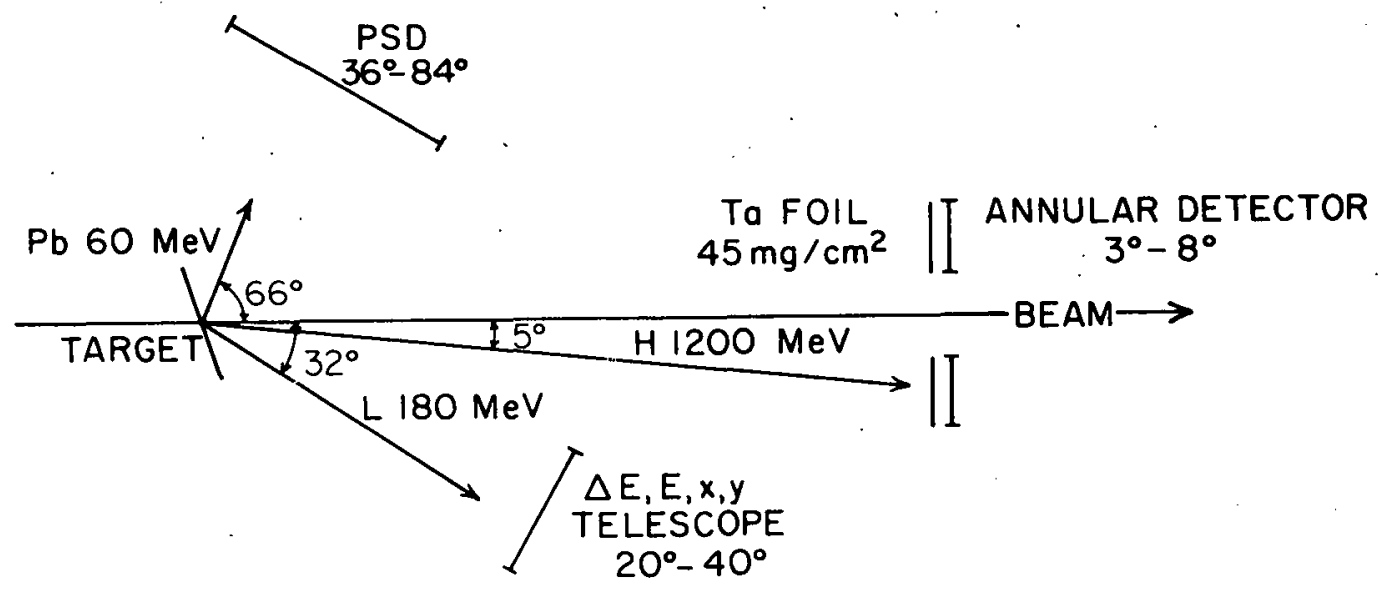

Fig. 6.4-1: The experimental arrangement. Angles and energies of reaction products expected for instantaneous fission at an. impact parameter of $1 \mathrm{fm}$ are indicated ( $\mathrm{H}$ : heavy fission fragment; L: light fission fragment).

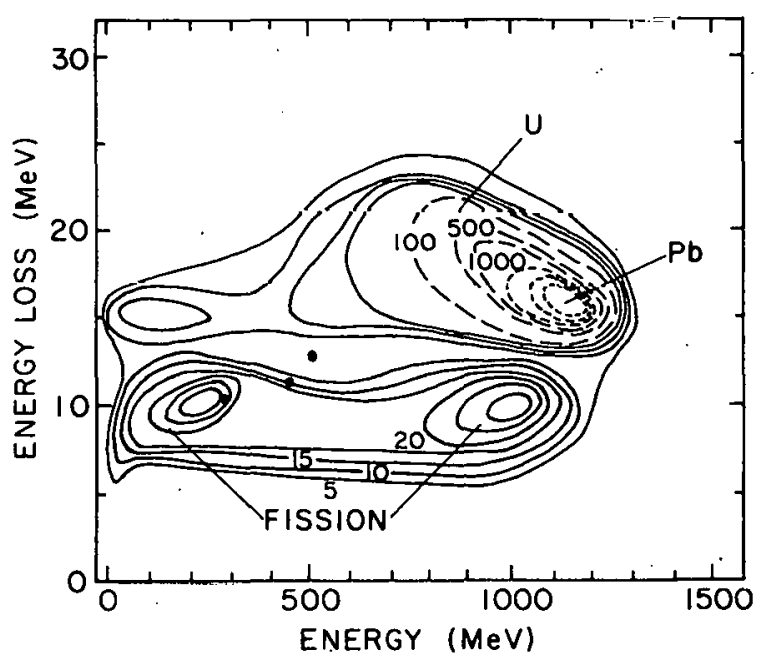

Fig. 6.4-2: Contour plot of energy loss versus residual energy of particles detected in the gas $\Delta E$, solid state $E$ telescope. Solid and dashed lines are spaced by an increase in intensity of 5 and 1000 counts, respectively. Full dots indicale events recorded in triple coincidence. 
If a projectile-like reaction product is registered in the position sensitive detector the detection efficiency for a triple coincidence--determined with a Monte Carlo calculation--is $90 \%$ in case of an instantaneous fission event for which all 3 reaction products are coplanar and $0.3 \%$ in case of a sequential fission event. The particle identification spectrum taken with the telescope is shown in Fig. 6.4-2. Apart from the two groups of fission fragments emitted forward and backward in the system of the moving $U$ nucleus, respectively, elastically scattered $\mathrm{Pb}$ and $\mathrm{U}$ nuclei are also observed. Three triple coincidences with events in the position sensitive detector at $\theta_{\mathrm{Lab}}=630,710$, and $72^{\circ}$ are indicated.

None of these events has the characteristics of instantaneous fission since the heavy fragment is registered in all cases in the lelescope and not in the annular detector. Considering the three events as an upper limit, a cross section of less than $0.5 \mathrm{mb}$ is deduced for instantaneous fission with the heavy fragment going to forward angles with high energy as predicted. This limit is two orders of magnitude smaller than the theoretically predicted cross sertion 3 nf $50 \mathrm{mb}$.

\section{References}

1. P. Dyer, K.J. Puigh, R. Vandenbosch, T.D. Thomas, and M.S. Zisman, Phys. Rev. Lett. 39, 392 (1977).

2. D. von Harrach, P. Glassel, Y. Civelekoglu, R. Manner, and H.J.

Specht, Phys. Rev. Lett. 42,1728 (1979).
H.H. Deubler and K. Dietrich, Phys. Lett. $62 \mathrm{~B}, 369$ (1976) and 2. Phys. A284, 2.37 (1978).

5. Measurement of the Non-Fusion Yield in $16_{0}+16_{0}$ at $\mathrm{E}_{\mathrm{cm}}=34 \mathrm{MeV}$

A. Lazzarini, H. Doubre, ${ }^{+}$K. Lesko, V. Metag, ${ }^{++}$A. Seamster, and

$R$. Vandenbosch

Time dependent Hartree-Fock calculations 1,2 predict that at sufficientiy high bombarding energies collisions between two heavy ions for small impact parameters do not lead to compound nucleus formation, hut rether procecd to deeply inelastic scattering with a total kinetic energy in the final state characteristic of the Coulomb barrier for the two nulcpi. At $E_{\text {ell }}=34$ MoV Koonin and Flanders have calculated that for the $16_{0}+16_{0}$ system the partial waves $\mathrm{L}-$ 0-6 do not lead to fusion. We have performed an experiment to measure the cross section for the ${ }^{16} 0\left({ }^{16} 0,16_{0 *}\right)^{16} 0^{*}$ reaction to determine whether the predictions of TDHF calculations are, in fact, borne out. Figure 6.5-1 presents the results of the TDHF calculations schematically.

Particle-particle coincidences were measured using two position sensitive gas $\triangle E$-solid state $E$ telescopes. In this manner, the final state was determined in a kinematically complete measurement. Angular distributions were measured for the inelastic yield from $\theta_{1 a b}=10^{\circ}-40^{\circ}$. 

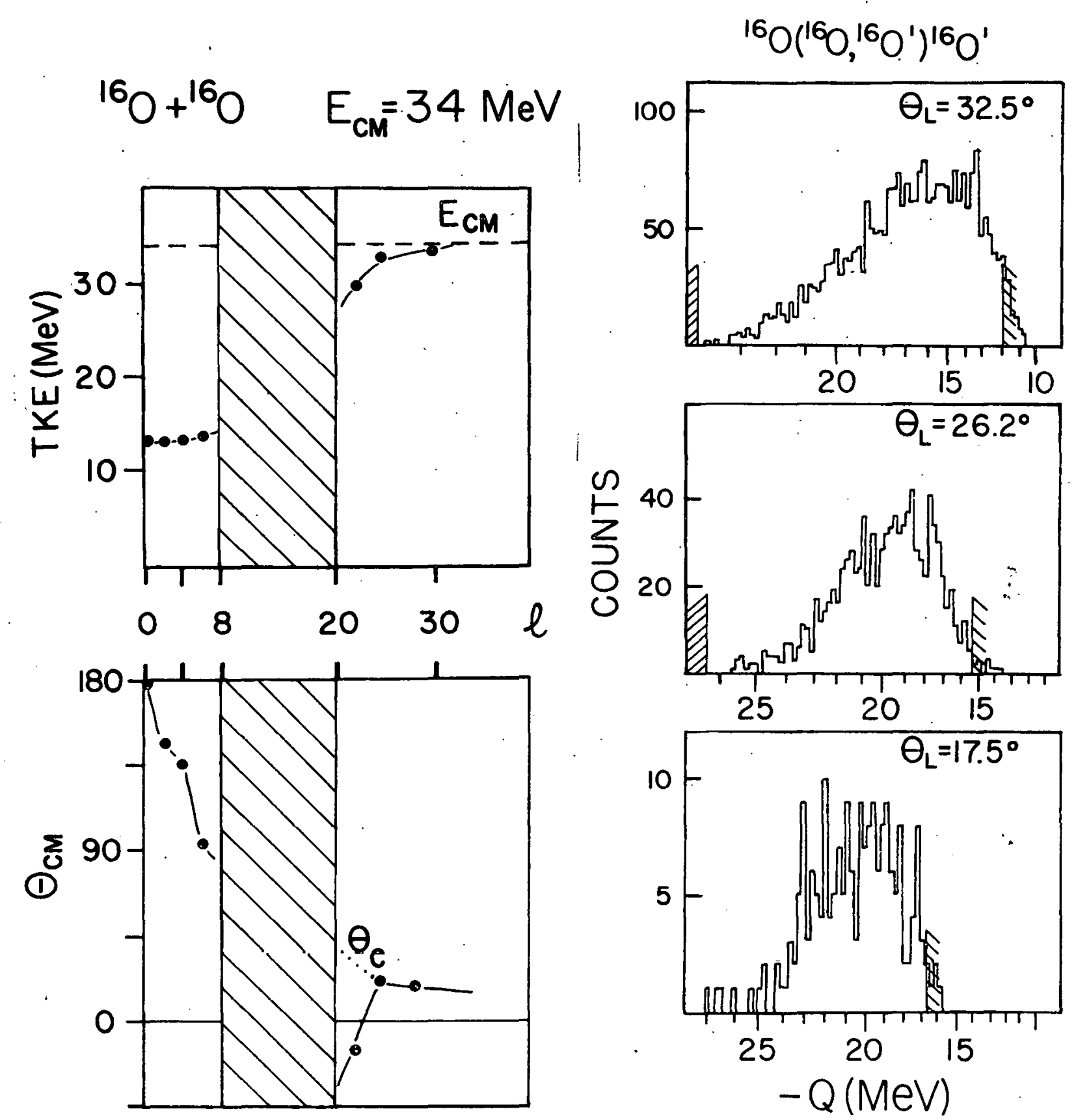

Fig. 6.5-1: TDHF calculations for $16_{0}+16_{0}$ at $E_{c m}=34 \mathrm{MeV}$ performed by Koonin and Flanders.

Fig. 6.5.2: Q-value spectra for one $16_{0}$ ion for $16_{0}+16_{0}$ coincidences at several laboratory angles. Energy scales are not linear because spectra are in laboratory frame. The hashed regions represent kinematic coincidence cutoffs. 
Figure 6.5-2 presents energy spectra for one reaction partner of the $16_{0}$ $+16_{0}$ coincidences. Because of the kinematic coincidence requirement the spectra contain limits in the reaction Q-values which could be measured. These are denoted by the diagonal hash-marks in each of the spectra. A broad inelastic bump which shifts with angle is apparent in the spectra. An enhancement of the inelastic yield in the region $-22 \mathrm{MeV}<Q<-20 \mathrm{MeV}$ which is predicted by the TDHF calculations as arising from the non-fusion of the inner partial waves is not observed here. This result is in agreement with what we determined in an earlier, less complete measurement that has been reported. Figure 6.5-3 shows the Wilczynski plot for this system. The dashed curve bounding the upper part of the contours represents the kinematic coincidence window. The entrance channel center of mass energy is marked by the dotted line. The $16_{0}+16_{0}$ Coulomb barrier is indicated by the dashed line labelled $\mathrm{V}_{\mathrm{C}}$. Several features are immediately obvinus: 1) The inelastic cross section for this reaction peaks at $\theta_{\mathrm{cm}}=90^{\circ}$. The symmetry about $90^{\circ}$ is imposed by the identical boson entrance channel. 2) A peak in the inelastic yield occurs for $Q$ $=-14 \mathrm{MeV}$. Even though the coincidence window closes rapidily for more positive Q-values, it is clear that the maximum cross section for $Q>-14 \mathrm{MeV}$ becomes significantly less than for $Q=-14 \mathrm{MeV}$.

Although the data are not in quantitative agreement with the theoretical predictions, they are qualitatively consistent. The agreement would be considerably improved if one assumed that the collison is not yet fully damped for the partial wave $L=6$. We note that the direction of this disagreement is opposite to that which has been reported in heavier systems where TDHF calculations have underpredicted the degree of damping. 3,4 The TDHF trajectory for the non-fusing partial waves $L=0,2,4,6$ is marked by the symbols " $x^{\text {' in }}$ Fig. $6.5-3$ (the impact parameter associated with $I_{1}=0$ scattcro to $0_{\mathrm{cm}}=0^{\circ}$ and that for $L=\dot{b}$ scatters to $\theta_{\mathrm{cm}}=87^{\circ}$ ). The increase of the inelastic yield towards $\theta_{\mathrm{cm}}=900$ is consistent with the fact that the partial wave $L=6$, which is predicted to scatter to $\theta_{\mathrm{cm}}=870$ carries the bulk of the deeply inelastic, non-fusing cross section. Assuming as usual a direct relationship for deep inelastic scattering between reaction $Q$-value and partial wave, $L$, the fact that we see a drop in the inelastic yield for $Q>-14 \mathrm{MeV}$ implies that the partial waves $L>6$ are proceeding to fusion.

The magnitude of the effect is in agreement with the TDHF predictions. $\sigma_{\mathrm{TDHF}}=132 \mathrm{mb}$ for $\mathrm{L}=0-6$, and we observe an experimental cross section, $\sigma_{\exp }=$ $200 \pm 50 \mathrm{mb}$. As a reference, the expected total reaction cross section is o $\sigma_{R}=$ $1450 \mathrm{mb}$ and the measured fusion-evaporation cross section is $\sigma_{\mathrm{F}}=1100 \mathrm{mb} .5^{\mathrm{R}} \mathrm{The}$ peaking of the inelastic cross section at $\theta_{\mathrm{cm}}=90^{\circ}$ is a novel result when one considers that for this light system the grazing angle is $\theta_{g}=200$. The angular dependence we observe is inconsistent with an interpretation that the deep inelastic yield arises from partial waves beyond the fuston limit $\mathrm{L}=20$.

The Wilczynski plot provides us with a tantalizing, yet still incomplete overview of the $16_{0}+16_{0}$ reaction. We hope to uncover more of this plane in an upcoming experiment. 
Fig. 6.5-3: Wilczynski plot for the $160+160$ system. Dashed lines represent kinematic coincidence window. $\theta \mathrm{g}$ is the grazing angle for the reaction. $\quad v_{C}$ is the $160+16_{0}$ Coulomb barrier.

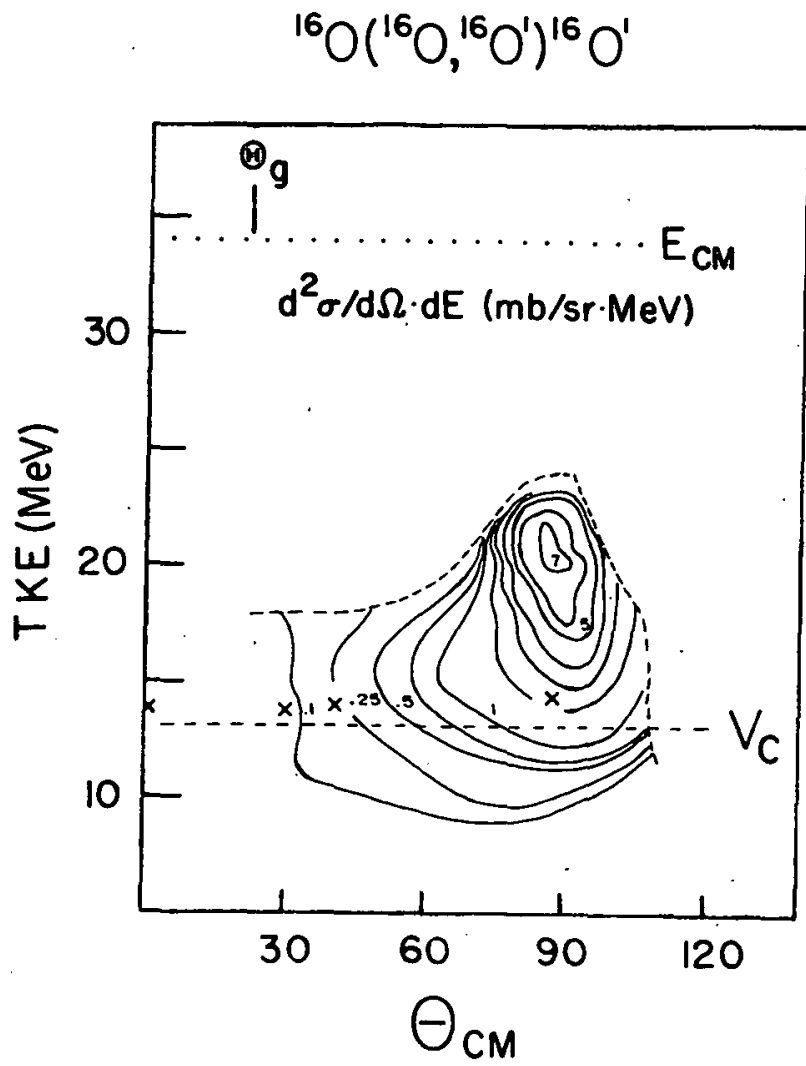

\section{References}

+ Present address: Institut de Physique Nucleaire, B.P. No. 1, Orsay 91406 France.

t+ On leave from: Max Planck Institüt für Kernphysik, D-6900 Heidelberg, P.o. Box 103980, West Germany.

1. H. Flocard, S.E. Koonin, and M.S. Weiss, Phys. Rev. C17, 1682 (1978). S.E. Koonin, B. Flanders, private communication.

3. R. Vandenbosch, M.P. Webb, P.Dyer, R.J. Puigh, R. -Weisfeld, T.D. Thomas, and M.S. Zisman, Phys.Rev C1.2, 1672 (1978).

4. K.T.R. Davies, K.R. Sandhya Devi, and M.R. Strayer, Phys. Rev. C20, 1372 (1979).

5. B. Fernandez, C. Gaarde, J.S. Larsen, S. Pontoppidan, and F. Videbaek, Nuc1. Phys. A315, 4'45 (1978). 
6. Fusion Excitation Function for $10_{B}+14_{N}$

H. Doubre, + A. Tazzarini, K. Lesko, E. Norman, A. Seamster, and

- R. Vandenbosch

The maximum fusion cross sections for the two systems $10_{B}+16_{O}$ and $12 \mathrm{C}+$ ${ }^{14} \mathrm{~N}$ have been reported to differ by $20 \%$ even though the entrance channels produce the same compound nucleus, $26 \mathrm{Al} .1$ similar, although less dramatic, differences have been reported more recently for the pair of systems $16_{0}+16_{0}$ and $12 \mathrm{C}+20 \mathrm{Ne}$ producing the compound nucleus $32 \mathrm{~s} .{ }^{2}$ If one calculates the maxinum angular momentum which contributes to fusion in a sharp cutoff approximation for the $26_{\mathrm{Al}}$ system, it is found that the curves defining the dependence of this maximum partial wave, $\ell_{c}$, with excitation energy of the compound system, $E^{*}$, are similar for both entrance channels. This behavior would indicate that the physics of the fusion process is determined by the compound nucleus rather than by the entrance channel. In this case, then, the tact that the maximum tusion cross section varies with entrance channel would reflect dynamic and/or kinematic effects.

We have measured the fusion-evaporation excitation function for the system $10_{\mathrm{B}}+{ }^{14} \mathrm{~N}$ from $\mathrm{E}_{1 \mathrm{ab}}=28-62 \mathrm{MeV}$. The compound nucleus, $24 \mathrm{Mg}$, has been much studied in this energy range via the entrance channel ${ }^{12} \mathrm{C}+{ }^{12} \mathrm{C}$. We intend to make a direct comparison of the maximum fusion cross sections for the two systems. Furthermore, since the ${ }^{12} \mathrm{C}+{ }^{12} \mathrm{C}$ system exhibits pronounced energy dependent structure in the excitation function, it is interesting to determine if any similar behavior occurs for the $10_{\mathrm{B}}+{ }^{14} \mathrm{~N}$ system. We are currently analyzing the data.

The experiment was performed using a position sensitive gas $\Delta^{E}$ solid state E proportional telescope based on the Markham design. 3 Using the position information together with a mask comprising of four aperatures, four different angles could be measured simultaneously for each counter position. Figure 6.6-1 shows a typical $\triangle E-E$ spectrum.

\section{References}

+ H. Doubre visiting here from Institut de Physique Nucleaire, ORSAY, France.

1. J. Gomez del Campo, R. $\Lambda$. Dayras, J.A. Biggerstaff, D. Shapira, A.H. Snell, P.H. Stelson, and R.g. Stokstad, Phys. Rev. Lett. 43, 26 (1979).

2. I. Tserruya, International Symposium on Heavy Ion Fusion Reactions, Bad Honnef, West Germany, March 10-13, 1980.

3. R.J. Markham, S.M. Austin, and H. Laumer, Nucl. Inst. and Meth. 129, $1.41(1975)$ : 


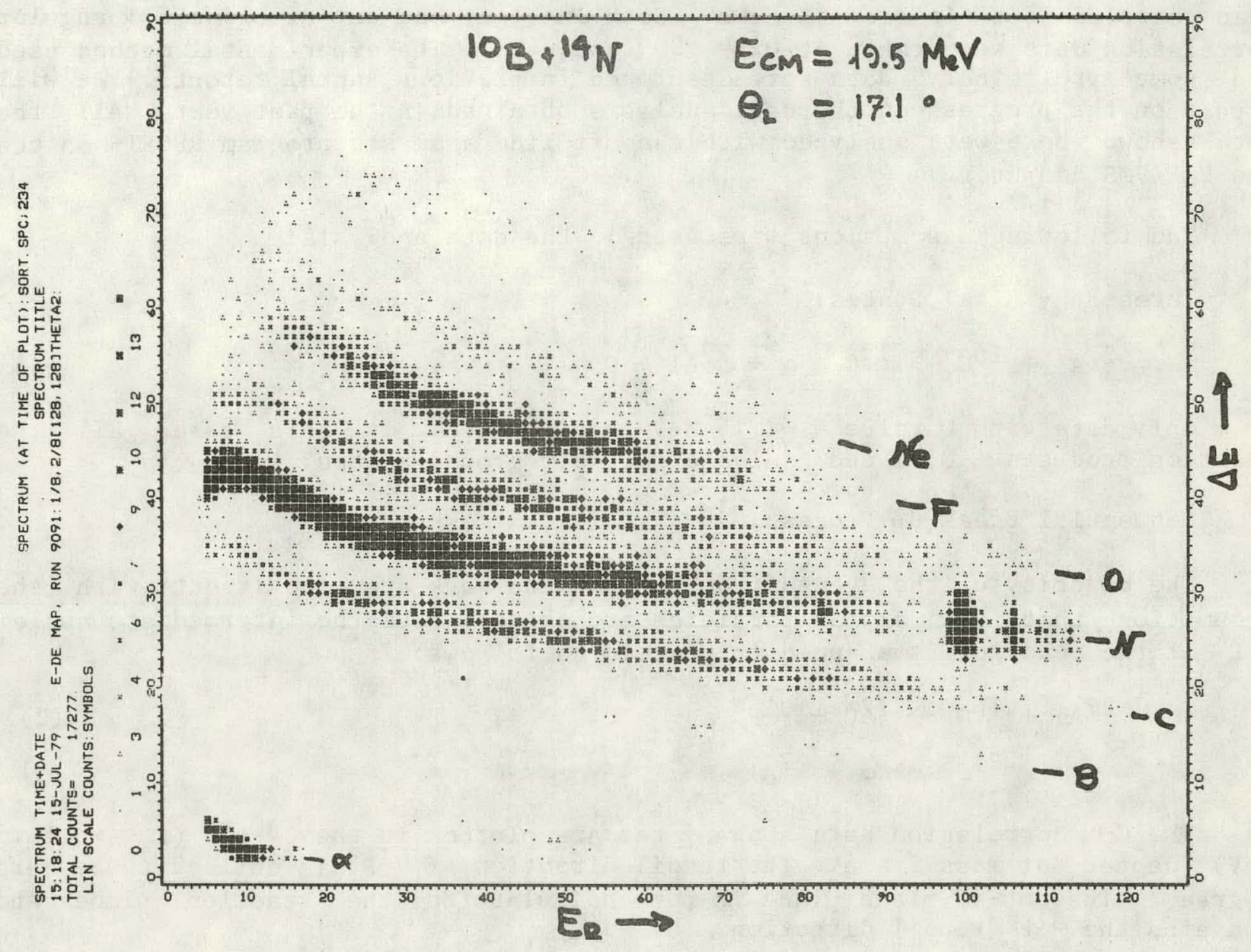

Fig. 6.6-1: $\triangle \mathrm{E}-\mathrm{E}$ plot for $10_{\mathrm{B}}+{ }^{14} \mathrm{~N}$ reaction obtained with a position sensitive gas $\Delta \mathrm{E}$ solid state $\mathrm{E}$ proportional counter. 
7. ${ }^{26} \mathrm{Al}\left({ }^{16} \mathrm{O},{ }^{12} \mathrm{C} \alpha\right){ }^{27} \mathrm{Al}$ Coincidence Study at $65 \mathrm{MeV}$

W.G. Lynch, R.J. Puigh, A.G. Seamster, M.B. Tsang, and R. Vandenbosch

\section{A. Introduction}

A comprehensive study on the coincidence reaction ${ }^{27} \mathrm{Al}\left({ }^{16} 0,{ }^{12} \mathrm{C} x\right){ }^{27} \mathrm{Al}$ has been carried out in recent years. Both in-plane and out-of-plane C- $\alpha$ angular correlation data were taken at $\theta_{c}=-30^{\circ}$ and $-40^{\circ}$. The experimental method used and some preliminary data were described in previous annual reports. ${ }^{1}$ We will report on the progress on the data analysis obtained in the past year. All the data shown here were analyzed with the off-line analysis program $\mathrm{RSORT}^{2}$ on the new VAX/VMS computer.

The following assumptions were used in the data analysis:

1. Three-Body Final States:

$$
{ }^{2 \prime} \mathrm{A} 1+{ }^{16} \mathrm{O} \rightarrow{ }^{12} \mathrm{C}+\alpha+{ }^{2 /} \mathrm{A} 1+\mathrm{Q}
$$

Only data with $Q$ value $=-7.16 \mathrm{MeV}$ is discussed. In this case, all the reaction products ${ }^{12} \mathrm{C}, \alpha$ and ${ }^{27} \mathrm{Al}$ are in their ground states.

2. Sequential Break-up Process:

The majority of the events from the present work are consistent with the assumption that the alpha particles are emitted from the intermediate nuclei ${ }^{31} \mathrm{P}^{*}$ if the reaction is assumed to proceed as follows:

$$
\begin{array}{r}
27 \mathrm{Al}+16 \mathrm{O} \rightarrow{ }^{12} \mathrm{C}+{ }^{31} \mathrm{P}^{*} \\
31_{\mathrm{P}^{*}} \rightarrow \alpha+{ }^{27} \mathrm{~A} 1
\end{array}
$$

The $\mathrm{C}-\alpha$ correlation data shown later are plotted in the $31 \mathrm{P}^{*} \quad\left(\mathrm{E}_{\mathrm{X}}=14.5\right.$ $\mathrm{MeV}$ ) center of mass frame. The recoil direction of $31_{\mathrm{P}}^{*}$ is defined to be zero degree. The out-of-plane plane is perpendicular to the reaction plane and contains the $31 \mathrm{P}^{*}$ recoil direction.

One of the goals of this work is to understand the nature of the pre-equilibrium alpha emission process. In order to extract out the pre-equilibrium component, all other processes that contribute to the alpha emission should be identified and subtracted.

B. Break Up Events from $160^{*}$

This process has been discussed briefly in last year's annual report.1 Such a mechanism can be represented as follows: 


$$
\begin{aligned}
& 160+27_{\mathrm{A} 1} \rightarrow{ }^{27} \mathrm{~A} 1+16_{0} * \\
& 16_{0} * \rightarrow \alpha+{ }^{12} \mathrm{C}
\end{aligned}
$$

In the present work, the $160^{*}$ break up process is observed at two angles $\theta_{\alpha}=-17^{\circ}$ and $\theta_{\alpha}=-43^{\circ}$ with $\theta_{c}=-30^{\circ}$.

Using kinematics, the excitation energy of oxygen $E_{o}$ for $\theta_{c}=-30^{\circ}$ and $\theta_{\alpha}=$ $-17^{\circ}$ can be deduced and it is found to be between 9.5 to $11 \mathrm{MeV}$. The lowest energy state in 160 that can decay by alpha emission is the one at $9.63 \mathrm{MeV}$. Proton decay starts to compete with the alpha decay above $12.1 \mathrm{MeV}$.

\section{Equilibrium Contribution}

Ericson and Strutinsky ${ }^{3}$ first showed that evaporation from a rotating nucleus can be treated classically. Halpern ${ }^{4}$ has developed a classical model for emission from a spherical rotating Maxwell gas. If a rotating nucleus is assumed to rotate around an axis that is perpendicular to the reaction plane, then one would expect the angular distribution of evaporated particles to be isotropic in the equatorial plane. Owing to the centrifugal force, the yield is concentrated in the equatorial plane and decreases as one goes towards the pole. If the rotating axis is normal to the reaction plane, then the equatorial plane is the reaction plane..

The yield of evaporation particles as a function of polar angle $\downarrow$ defined with respect to the axis of rotation is given by Halpern:

$$
Y(\psi)=Y_{0} \exp \left(X * \sin ^{2} \psi\right)
$$

where $Y_{0}$ is a normalization factor. $X$ is the ratio of rotational kinectic energy to the thermal nuclear energy,

$$
X=\frac{\frac{1}{2} \mu R^{2} L T^{2}}{2 T}
$$

where $\mu$ is the reduccd mass; Wf is the angular velocity and $T$ is the nuclear temperature. The radius $R$ is taken as

$$
R=1.25\left(A d 1^{3}+A_{\alpha}^{1 / 3}\right)
$$

$X$ can also be expressed in terms of the spin $(\mathrm{J})$ of the rotating nucleus:

$$
x=\frac{\frac{1}{2}\left(J+\frac{1}{2}\right)^{2} \hbar^{2}}{2 I T}
$$


where $I=\mu R^{2}$ is the moment of inertia.

The $c-\alpha$ correlation function plotted in the $31 \mathrm{P}^{*}$ center of mass frame shows a slight backward angle rise. This leads to the conclusion that the rotating axis is not perfectly aligned along the $z$ axis which is normal to the reaction plane. Simple argument suggests that the angular momentum of the $31_{P^{*}}$ is expected to lie in the plane perpendicular to the reaction plane and the momentum transfer direction. The momentum transfer direction 1 s assumed to be the recoil direction of $31 \mathrm{P}^{*}$. The angle between the rotational axis and the $z$ axis is defined to be $\gamma$. In order to estimate the contribution of the evaporation component in a simple way, $\gamma$ is assumed to be gaussian distributed. The coordinate system used is shown in Fig: 6.7-1. It is chosen to simplify the evaporation analysis and differs from that often used.

From Equation 6.7-3, the angular distribution is

$$
W(\theta, \phi)=\int_{0}^{2 \pi} d \gamma \exp \left(-\gamma^{2} / 2 \gamma_{0}^{2}\right) Y(\psi)
$$

where $\Phi$ is related to the angles $\gamma, \theta$, and $\phi$ as shown in Fig. $6.7-1$ by the cosine law:

$$
\cos \phi=\cos \gamma \cos \phi+\sin \gamma \sin \phi \sin \theta
$$

The angular distribution $w(\theta, \phi)$ used in equation $6.7-10$ is the same as the experimental quantity $\left(\mathrm{d}^{2} \sigma / \mathrm{d} \Theta_{c} \mathrm{~d} \Theta_{\alpha}\right) \mathrm{cm}$, the double differential cross-section of the coincidence $C-\alpha$ events. In order $L u$ empliasize the $\theta$ and $\phi$. Aependenre of this quantity, the more convenient notation $w(\theta, \phi)$ is adopted.

Both the out-of-plane data and the in-plane data are used to determine the two parameters $\gamma_{0}$ and $X$. The dependence of $Y_{0}$, the normalization constant of Equation $6.7-6$, is eliminated by actually fitting the calculated ratio

$$
\frac{W(\theta, \phi)}{W(0,90)}=\frac{\int_{0}^{2 \pi} d \gamma \exp \left(-\frac{\gamma^{2}}{2 \gamma_{0}^{2}}\right) \exp \left[X \sin ^{2} \psi\right]}{\exp (X) \int_{0}^{2 \pi} d \gamma \exp \left(-\frac{\gamma^{2}}{2 \gamma_{0}^{2}}\right)}
$$

to the same ratio obtained experimentally. 


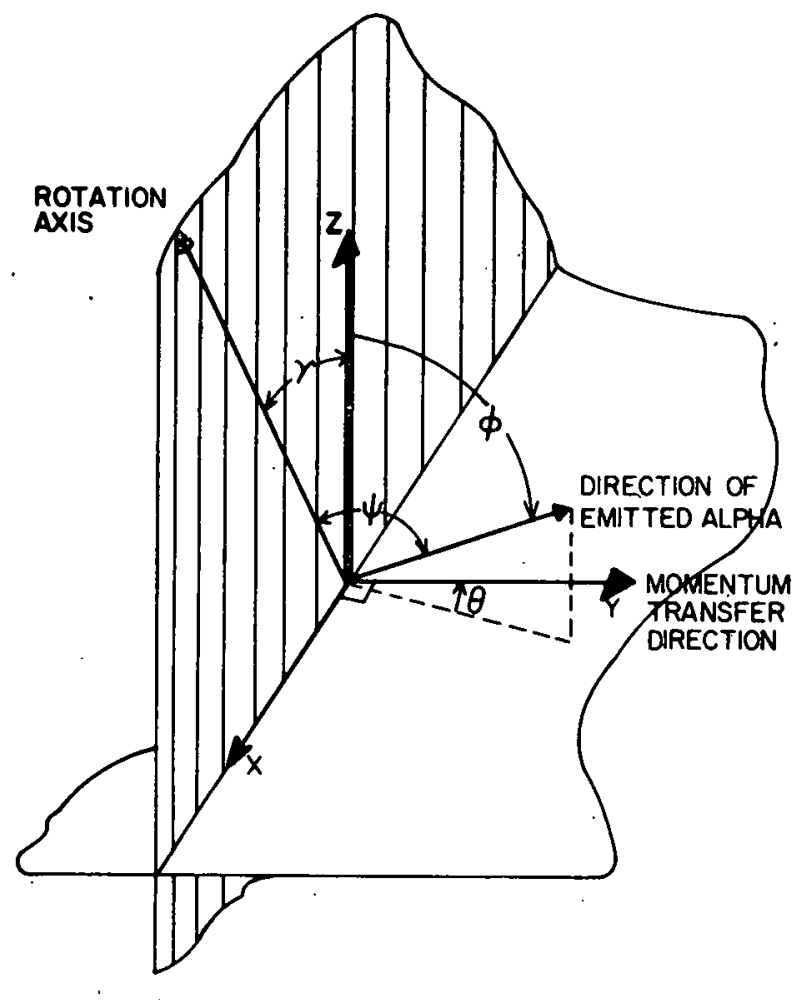

Fig. 6.7-1: Coordinate system used in describing a rotating ${ }^{31} \mathrm{P}^{*}$ nucleus emitting an alpha particle.

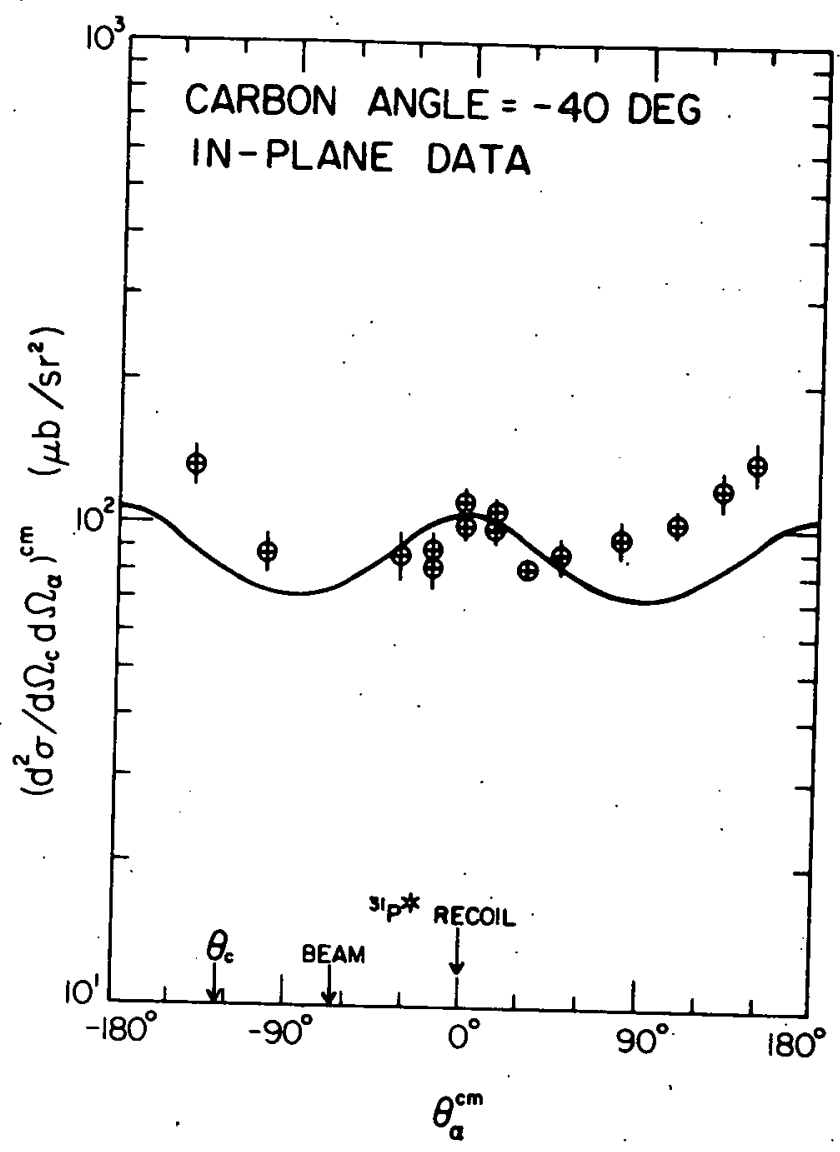

Fig. 6.7-2: In-plane $c-\alpha$ angular correlation function in the $31_{\mathrm{P}}$ center of mass system, $\theta_{c}=-40^{\circ}$. The solid line is the best fit of Equation (6).

The solid lines in Figs. 6.7-2 and 6.7-3 are the best fit of the in-plane and out-of-plane $C-\alpha$ correlation data plotted in the $31_{P^{*}}$ center of mass frame for $\theta_{c}=-40^{\circ}$. Best $X^{2}$ values for $\gamma_{0}$ and $X$ are found to be $28^{\circ}$ and 3.1 , respectively. The in-plane data is relatively featureless and would have been consistent with an evaporative origin if not for the large rise at backward angles. It is unfortunate that more data is not available at these angles. 


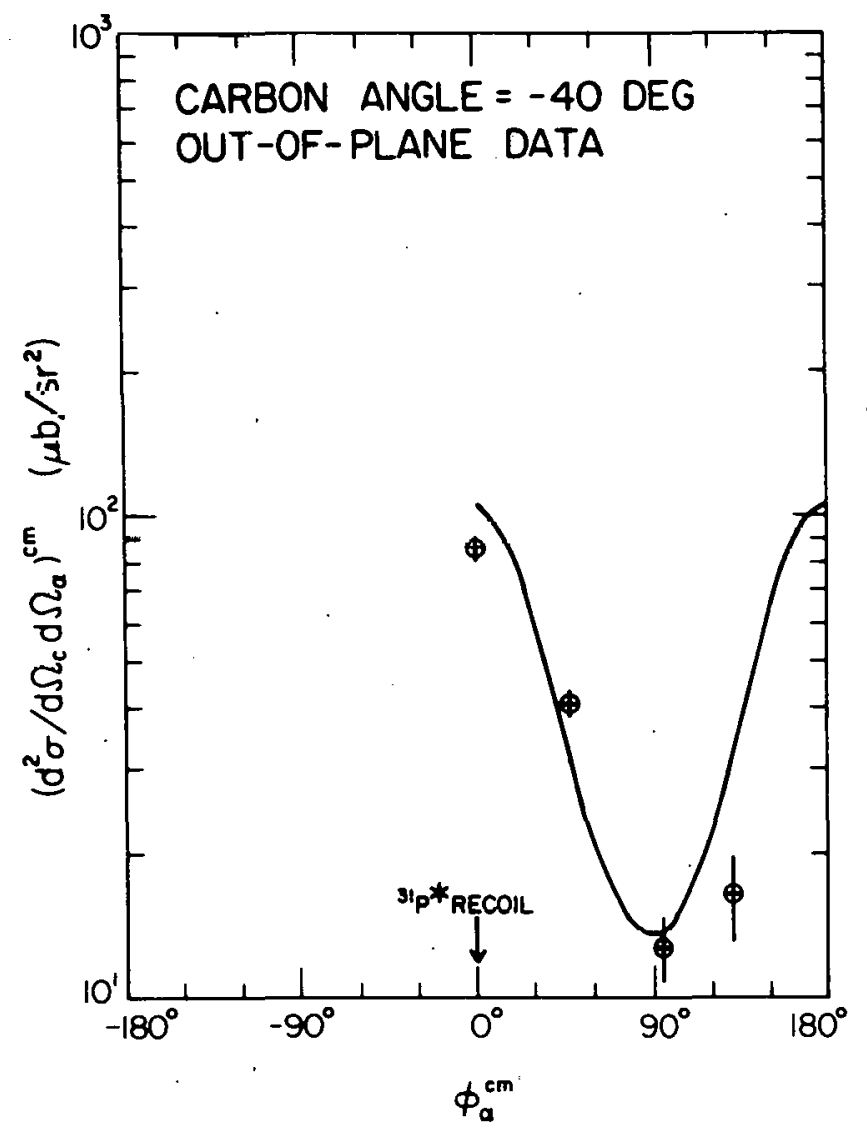

Fig. 6.7-3: Out-of-plane $c-\alpha$ angular correlation function in the ${ }^{31} \mathrm{P} *$ center of mass system, $\theta_{C}=-40^{\circ}$. The solid line is the best fit of Equation (6).

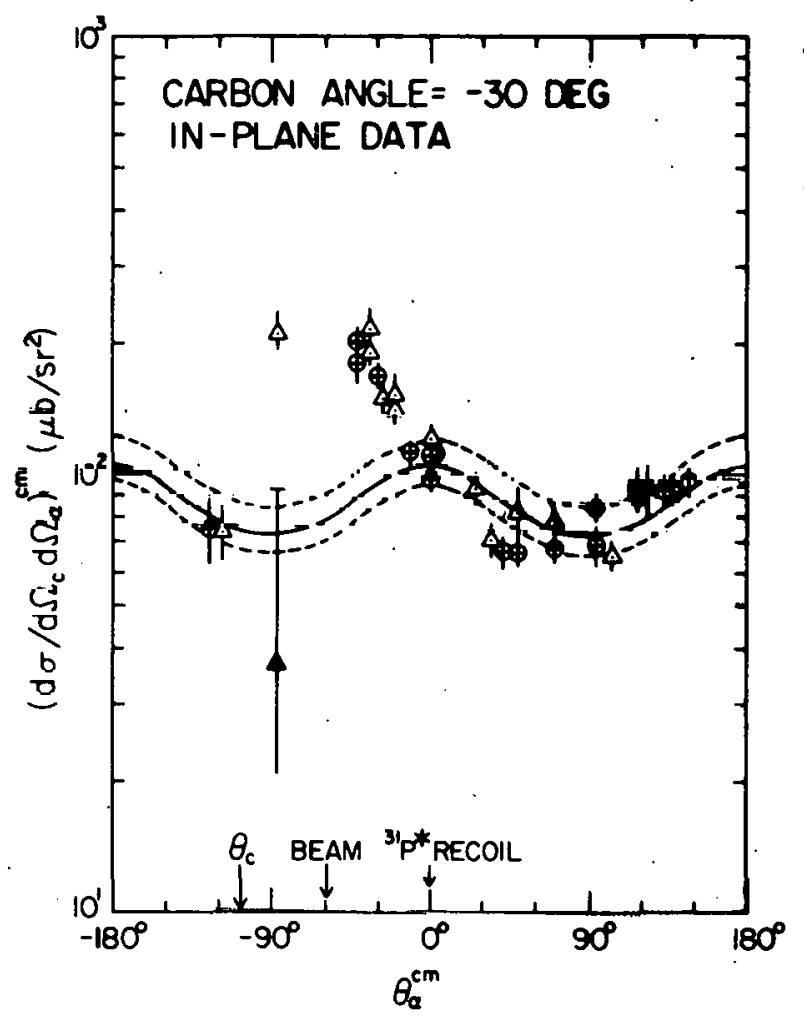

Fig, 6.7-4: In-plane $c-\alpha$ correlation function in the $31_{\mathrm{P}} *$ center of mass system $\theta_{c}=-30^{\circ}$. Upen $\mathrm{rr1-}$ angles are data taken with the two-telescope method and circles are data taken with the time-offlight method. All the data are normalized with respect to each other uding the single events detected by the carlion telescope.

Figures 6.7-4 and 6.7-5 are the in-plane and out-of-plane $c-\alpha$ angular cortelation for $\theta_{c}=-300$ plotted in the $31_{\mathrm{P}^{*}}$ center of mass frame. The solid triangle with large error bars at $\theta_{\alpha}^{c m}=-90^{\circ}$ is obtained after the $16_{0 *}$ break up contribution is subtracted. The solid lines are the best fit obtained by using only backward angles $\left(\theta_{\alpha}^{c m}>90^{\circ}\right)$ in-plane data along with the out-of-plane data since the evaporative component is expected to dominate at backward angles. The $\gamma_{0}$ and $X$ values obtained are very close to those obtained for $\theta_{c}=-40^{\circ}$ data, $\gamma_{0}=280$ and $x=2.6$. Only the in-plane data from $-30^{\circ}<$ $\theta_{\alpha}^{c m}<0^{\circ}$ are not fitted by this purely evaporative model. All other angles are seen to be fitted very well by the simple evaporative angular correlation. 


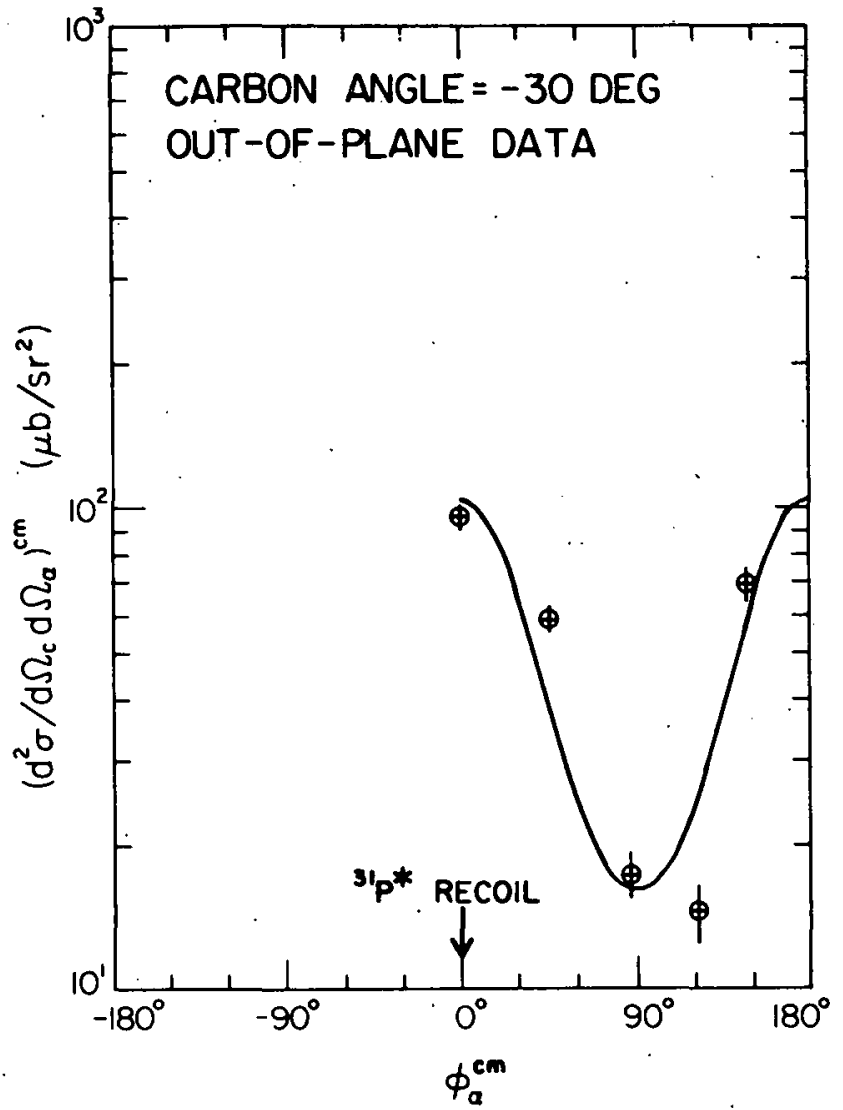

Fig. 6.7-5: Out-of-plane $c-\alpha$ angular correlation function in the center of mass system of $31_{p^{*}}, \theta_{c}=$ $-30^{\circ}, \theta_{\alpha}=43^{\circ}$. The $31_{\mathrm{P} *}$ recoil direction in the reaction plane is defined to be $\phi_{\alpha}=0^{\circ}$. The solid line is the best fit of Equation (6).

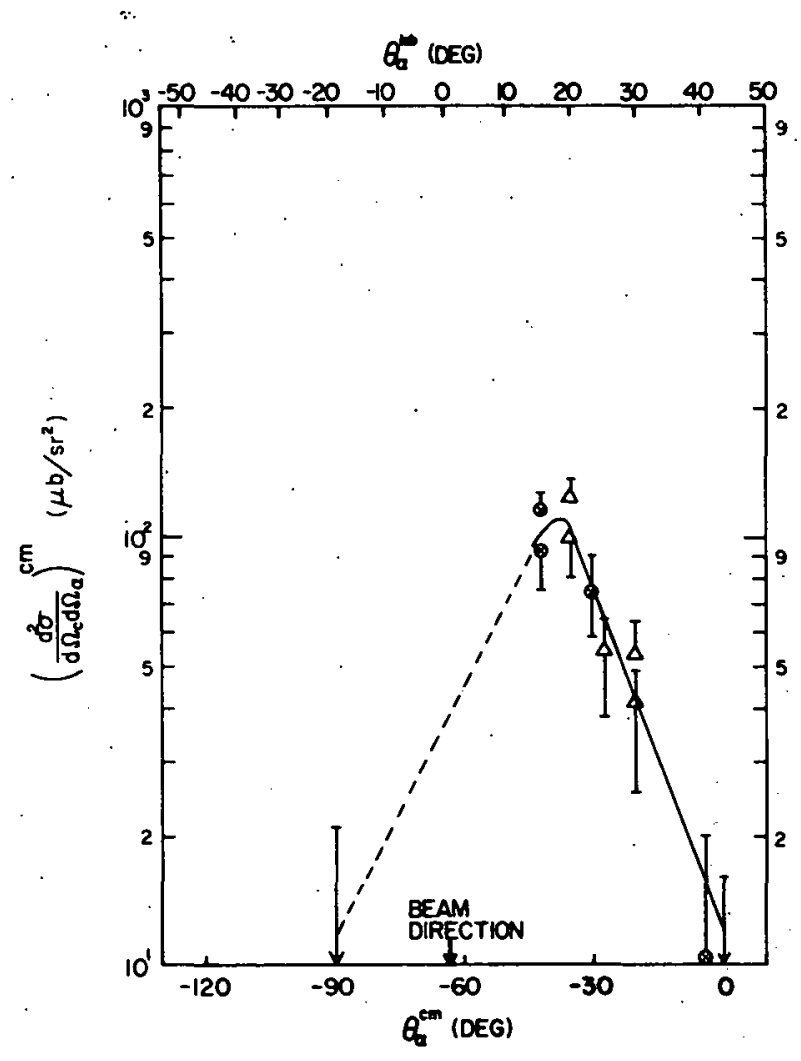

Fig. 6.7-6: Extracted pre-equilibrium alpha double differential crosssection plotted as a function of alpha angles in the ${ }^{31_{P *}}$ center of mass system. The corresponding lab. angles are also given.

From the values obtained for $\gamma_{0}$ and $X$, one can estimate the spin of the nucleus to be $J=7 . h$ using Equations $6.7-7,6.7-8$ and 6.7-9.

D. Pre-equilibrium Contribution

Since the in-plane and out-of-plane $C-\alpha$ angular correlation at $\theta_{c}=-40^{\circ}$ are consistent with evaporation, it can be assumed that the data discussed in this section was taken at $\theta_{c}=-30^{\circ}$ unless otherwise specified. 
Fig. 6.7-7: Mean $\alpha$ energy plotted as function of $\alpha$ angle in the $31_{\mathrm{P}}$ * center of mass system. The straight line denotes $\left\langle\mathrm{E}_{\alpha}^{\mathrm{cm}}\right\rangle=$ $5.2 \mathrm{MeV}$.

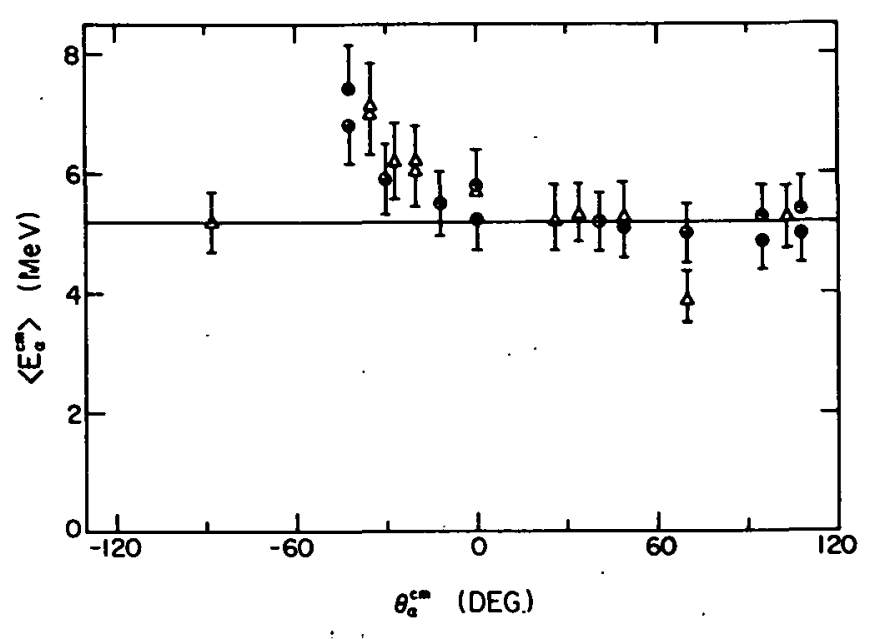

As explained in the last section, the evaporative component was extracted by fitting Equation 6.7-10 to the back angle data. The beyl fit is shown ac a solid line in Fig. 6.7-4. The dotted lines above and below the solid line represent upper and lower limits for the evaporative component by adjusting the normalization factor $Y_{0}$ in Equation 6.7-6. The pre-equilibrium alpha emission contribution is operationally defined here to be the experimental yield minus the yield from evaporation and the break up of $160^{*}$. It is plotted in the center of mass frame of $31_{\mathrm{P}^{*}}$ in Fig. 6.7-6. The corresponding lab angles are given on the top axis. Since the value of the Jacobian does not change very much over this range, the pre-equilibrium component would show very similar features in the lab frame.

The mean alpha energy in the center of mass frame of ${ }^{31} 1^{*}\left\langle\mathrm{E}_{\mathbb{X}}^{\mathrm{cm}}\right\rangle$ is plotted as a function of alpha angle in Fig. 6.7-7. The data points at the most backward al pha angles were excluded since they suffer from low alpha energy cutoff problem. Over most angles, $\left\langle E_{\alpha}^{c m}\right\rangle$ is constant as expected if the alpha particles come from the evaporation of $31 \mathrm{P}^{*}$. For the forward angles where the pre-equilibrium alpha emission is important, $\left\langle E_{\alpha}^{\mathrm{cm}}\right\rangle$ is much higher at these angles than the $\left\langle E_{\mathcal{O}}^{\mathrm{Cm}}\right\rangle$ obtained at the back angles. The mean alpha energy of the pre-equilibrium component for each alpha angle can be estimated in the following way:

$$
\left\langle\mathrm{E}_{\alpha}^{\mathrm{cm}}>=\mathrm{f} x<\mathrm{E}_{\alpha}^{\mathrm{cm}}>\text { pre }+(1-\mathrm{f}) \mathrm{x}<\mathrm{E}_{\alpha}^{\mathrm{cm}} \mathrm{eq}_{\text {eq }}\right.
$$

where $f$ is the fraction of pre-equilibrium alpha particles emftted. (Licm; is the average energy for the equilibrium component and can be determined from Fig. $6.7-7$ to be $5.2 \pm 0.5 \mathrm{MeV}$. Then $\left\langle E_{\mathrm{CX}}^{\mathrm{Cm}}\right\rangle$ pre is the average energy for the pre-equilibrium alpha and can be determined from Equation 6.7-13 and is found to be $8 \pm 2 \mathrm{MeV}$. $\left\langle\mathrm{E}_{\mathrm{OX}}^{\mathrm{Cm}}\right\rangle$ pre is found within the uncertainties to be angle independent at all alpha angles $\left(\theta_{\alpha}^{\mathrm{cm}}=15^{\circ}, 20^{\circ}, 25^{\circ}, 30^{\circ}\right)$ where the pre-equilibrium component is present. The large error in $\left\langle\mathrm{E}_{\alpha X}^{C m}\right\rangle_{\text {pre }}$ is mainly due to the uncertainty in determining $f$. 
E. Summary

The results from the present work can be summarized as follows:

1) Very few if any pre-equilibrium alpha particles are detected in coincidence with carbon particles detected at $\theta_{c}=-400$. The $C-\alpha$ angular correlation function is consistent with that of evaporation from ${ }^{31} \mathrm{P}^{\star}$.

2) At $\theta_{1}=-300$, the majority of $C-\alpha$ conicidence events come from evaporation of $31_{\mathrm{P}^{*}}$. A small amount of alpha particles from the break up of $16_{0}^{*}$ are detected at angles around the carbon detector. From the in plane $C-\alpha$ angular correlation shown in Fig. 6.7-4, one can estimate that less than $10 \%$ of the alpha particles detected in-plane are of pre-equilibrium origin. This result and the $C-\alpha$ angular correlation obtained at $\theta_{c}=-30^{\circ}$ are significantly different from the earlier results obtained by Harris et al. ${ }^{5}$

3) The pre-equilibrium alpha particle distribution extracted is forward-peaked on the opposite side of the beam direction as the carbon detector.

4) The average energy of the pre-equilibrium alpha particles are higher than that of the equilibrium alphas.

\section{References}

1. Nuclear Physics Laboratory Annual Report, University of Washington (1978), p. 65; (1979), p. 40.

2. Nuclear Physics Laboratory Annual Report, University of Washington (1979), p. 164 .

3. Ericson, T.E.O. and V.M. Strutinski, Nucl. Phys. $\underline{8}, 284$ (1958); $\underline{9}, 689$ (1959).

4. I. Halpern, private communication.

5. J.W. Harris et al., Phys. Rev. Lett. 38, 1460 (1977).

8. Deviation from Rutherford Scattering for Heavy Ions at Energies Far Below the Coulomb Barrier

T. Bertram, H. Bhang, J. Cramer, D. Leach, W. Lynch, R.J. Puigh and M.B. Tsang

Last year ${ }^{1}$ we reported on an investigation of deviations from Rutherford scattering for heavy ions at energies far below the Coulomb barrier. We pointed out that deviations from Rutherford scattering can be caused by several sources: 1) the use of a relativistic wave equation instead of the Schrofinger equation, 2) alteration of the Coulomb potential by the creation of virtual electron-positron pairs, and 3) polarization of the nuclear charge distributions by the repulsive Coulomb potential. 
In the same report we presented preliminary data on the systems ${ }^{12} \mathrm{C}+{ }^{208} \mathrm{~Pb}$ and $160+208 \mathrm{~Pb}$. These experiments were performed with detectors fixed at $\pm 30^{\circ}$, $\pm 140^{\circ}, \pm 150^{\circ}, \pm 160^{\circ}$, and $\pm 170^{\circ}$. The data were presented in the form of the ratio of the sum of the eight backward angle peak areas divided by the sum of the peak areas at $\pm 30^{\circ}$. (This experimental ratio is for most purposes an unnormalized measurement of the ratio $\sigma\left(150^{\circ}\right) / 0\left(30^{\circ}\right)$.) The absolute magnitude of this ratio has no significance; what is significant is the energy dependence of the ratio: For Rutherford scattering, this ratio should be energy independent. Instead the ratio obtained experimentally decreased with increasing energy. The data is presented: in Fig. 6.8-1. Each excitation function has an arbitrary overall normalization, which has been adjusted to make the excitation functions coincide. The abscissa of the plot is the classical turning point of the $30^{\circ}$ trajectory:

$$
r_{\min }=\frac{\mathrm{Z}_{\mathrm{p}_{\mathrm{t}} \mathrm{Z}^{2}}}{2 \mathrm{E}_{\mathrm{cm}}}(1+\csc [300 / 2])
$$

where $z_{p}, z_{t}$ are the charges of the projecrile, rargel lespectively. $E_{c m}$ is tho energy in the center of mass system.

This year similar excitation functions have been measured for the systems $16 \mathrm{O}+208 \mathrm{~Pb},{ }^{14} \mathrm{~N}+208 \mathrm{~Pb}$, and ${ }^{15} \mathrm{~N}+{ }^{208} \mathrm{~Pb}$. These three excitation functions were taken with the same experimental setup so their relative normalizations are not arbitrary but can be determined from kinematics. These data are plotted in Fig. 6.8-2 where the overall normalization has been adjusted to agree with Fig. $6.8-1$.

It is clear that there is an unambiguous projectile independent trend to the data. We are doing more detailed studies of the systematic errors which can alter the slope of the excitation function. With the exception of multiple scattering, the systematic errors appear to be insignificant. For multiple scattering, however, the systematic corrections may end up increasing the magnitude of the low energy data points as much as a few tenths of a percent, thereby increasing the slope slightly.

Through numerical studies comparing classical mechanical cross section calculations with quantum mechanical calculations we have seen that the classical approach is sufficiently accurate to. predict the deviations from Rutherford scattering for heavy ion scattering. Therefore we have written a program based on classical mechanics to calculate the deviation from Rutherford scattering where all the perturbing potentials are treated simultaneously.

The effects of: 1) use of a relativistlc wave equation; 2) vacuum polarization potential, and 3) nuclear polarization are shown by the dashed curve in Fig. $6.8-2$. It is clear that these three effects are not adequate to explain the data; particularly the deviation from Rutherford scattering at large $r_{\text {min }}$, i.e., low-energies. 


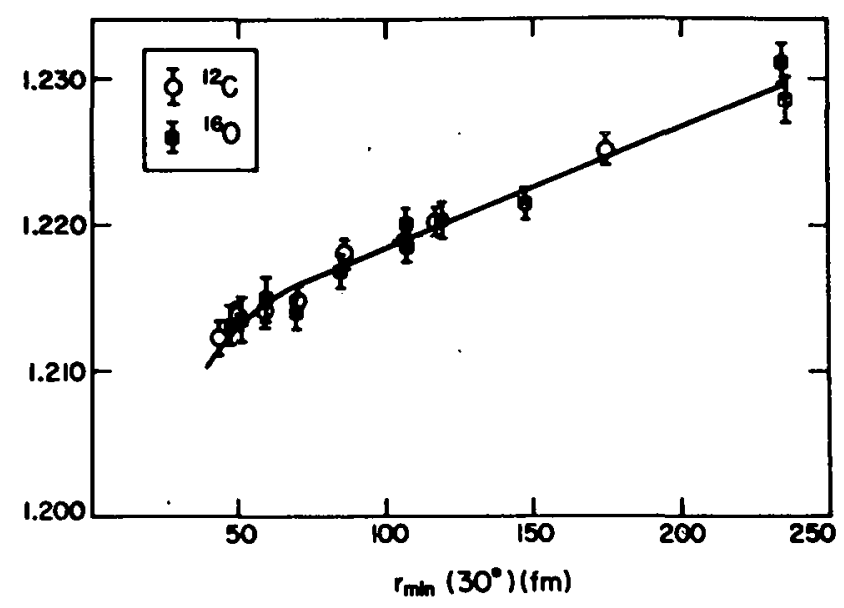

Fig. 6.8-1: $\quad{ }^{12} \mathrm{C}+{ }^{208} \mathrm{~Pb}, 16_{\mathrm{O}}+208_{\mathrm{Pb}}$ data reported on last year. Solid curve is a calculation predicting the effects of using a relativistic wave equation plus the vacuum polarization potential, nuclear polarization potential, and electron screening usine Hartree-Fock electronic wavefunctions.

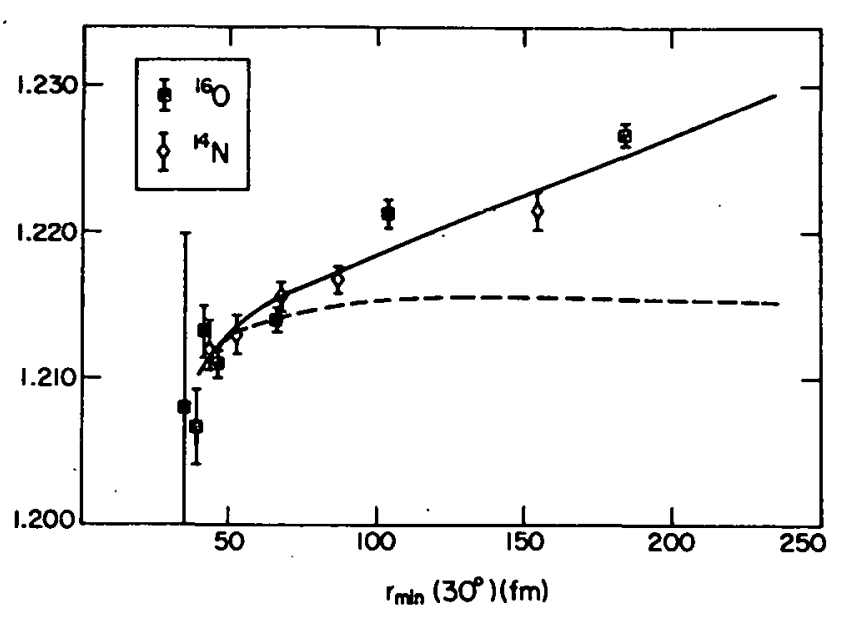

Fig. $6.8-2: \quad{ }^{14} \mathrm{~N}+208 \mathrm{~Pb}, 16 \mathrm{O}+208 \mathrm{~Pb}$ data taken this year. Solid curve is a prediction for $\sigma\left(150^{\circ}\right) / \sigma\left(30^{\circ}\right)$ which takes into consideration the effects of electronic screening, relativistic wave equation, vacuum polarization and nuclear polarization. The dashed curve is a similar calculation to the solid curve with the difference that the effects of electronic screening have been neglected.

However, when we include the effects of screening due to the inner-shell electrons around the target nucleus, the agreement is improved dramatically. The solid curve in Figs. $6.8-1$ and $6.8-2$ shows the result of a calculation similar to that described above in which the electron screening potentials of Vogel ${ }^{2}$ and of $\mathrm{Lu}$, et al. ${ }^{3}$ have been included.

It is clear that qualitative agreement between theory and experiment have been achieved. We are working to incorporate all small systematic corrections to the data in order to enable us to make quantitative comparisons between theory and experiment at the level of our statistical accuracy. It is hoped that when this is accomplished it will be possible to focus on the effects due to nuclear polarization and those due to the use of a relativistic equation.

\section{$\underline{\text { References }}$}

1. Nuclear Physics Laboratory Annual Report, University of Washington (1979), p. 93 .

2. P. Voge1, Atomic Data and Nuclear Data Tables, 14, 600 (1974).

3. C.C. Lu et al., Atomic Data, $\underline{3}$ (1971), 1 . 
J.S. Blair

Two proposals to measure reaction times in heavy-ion collisions have recently been put forward extending the earlier work of Ciocchetti and Molinari.l In these the reaction time is related to oscillatory structure either in the energy spectrum of $\delta$-electrons ${ }^{2}$ or in the probability for producing $\mathrm{K}$-shell vacancies as a function of impact parameter. 3 The crucial ingredient in both proposals is the interference between ionization amplitudes for incoming and outgoing trajectories. Specifically, the probability for exciting an electron in the lowest orbital into a continuum state with kinetic energy $\varepsilon$ is derived, in all three references, to have the form

$$
\left|a e^{-i \omega j}+b\right|^{2}
$$

Here $a$ and $b$ are semi-classical approximations to the amplitudes for ionizing an electron on the "way in" and on the "way nut", respecllvely, and $\hbar \omega=I+\varepsilon$. where $I$ is the binding energy of a K-shell electron of the united atom. The derivations leading to this result have a crucial deficiency, however in that their treatment of reaction times and the relative motion of the heavyions is purely classical.

This deficiency was encountered previously when we considered testing the proposals of Ciocchetti and Molinari through the scattering of low energy protons by nuclei. ${ }^{4}$ Here we found a number of paradoxes … and puzzles as we attempted to apply the semi-classical theory. All of these arose from employing a theory in which the notion of nuclear "time delay" was not related to any quantum-mechanical description of the nuclear reaction. To avoid these pitfalls, we constructed a completely quantum-mechanical description of the electron-nuclear system during proton-nucleus scattering and consequently derived 4,5 the following expression for the probablllty for ionizing a K-shell electron of the target nucleus while elaslically scatcoring the proton into àngle $\theta$ :

$$
|[f(E-\hbar \omega) / f(E)] a+b|^{2} \text {. }
$$

Here $f(E)$ is the amplitude for elastically scattering a proton into angle $\theta$ and $a$ and $b$ are the same ionization amplitudes which appear in the semi-classical theory of Ref. 1. This result is strikingly similar to the semi-classical expression, eq. (1); it is seen that the phase factor of the semi-classical theory is replaced by the ratio of scattering amplitudes evaluaced at differing bombarding energies.

A fully quantum-mechanical, time-independent description of ionization can also be constructed for a model of a heavy-ion collision. Two spin zero nuclei coljide and are assumed to produce binary fragments with charges, angular momenta, and energies, $Z_{1}^{\prime}, Z_{2}^{\prime}, I_{1}^{\prime}, I_{2}^{\prime}, E_{1}^{\prime}, E_{2}^{\prime}$, respectively. We consider only monopole excitation of an electron in the lowest molecular orbital to an ionized state with energy - For nuclear separations less than a matching radius $R_{m}$, we assume the electron wave functions to be those of a united atom. For nuclear 
separations larger than $R_{m}$ we assume that there are no nuclear interactions; this implies neglect of the long range Coulomb excitation of the nuclei. Keeping only 1st-order contributions to the ionization, using the WKB approximation for the relative radial motion of the separated nuclei, and matching inside and outside solutions at $R_{m}$, we derive the following formula for the joint differential cross section for producing the binary fragments and simultaneously ionizing the electron

$$
\left.\sum_{I^{\prime}, M^{\prime}} \sum_{L, L^{\prime}}\left(a(L) S_{L^{\prime} I^{\prime}}^{L}\left(E-\hbar^{\prime} J\right)+b\left(L^{\prime}\right) S_{L^{\prime} I^{\prime}}^{L}(E)\right)^{\frac{\sqrt{\pi}}{K}} \sqrt{2 L+1}\left(I^{\prime} L^{\prime} M^{\prime}-M^{\prime} \mid L 0\right) Y_{L^{\prime}}^{M^{\prime}}\right|^{2}
$$

Here $S_{L}^{L} I^{\prime}$ is an element of the S-matrix; $L$ and $L^{\prime}$ are the incident and final orbital angular momenta while the channel spin $I^{\prime}$ is the vector sum of $I_{1}^{\prime}$ and $I \frac{1}{2} ; \quad E$ and $K$ are the $c$. of $\mathrm{m}$. energy and wave number of the incident heavy ions at $R=R_{m}$; $\hbar \omega$ is the electronic energy difference for the united atom; $a(L)$ and $b(L)$ are equivalent to the incoming and outgoing ionization amplitudes of the semi-classical theory with impact parameter $L$ and $L^{\prime}$. If we assume that the nuclear partial wave amplitudes are concentrated around $L$ and $L^{*}$, we may factor out the ionization amplitudes and find that the joint cross section equals

$$
\sum_{I^{\prime}, M^{\prime}}\left|a(\bar{L}) f_{I^{\prime}, M^{\prime}}(E-\hbar \omega)+b(\bar{L}) f_{I^{\prime}, M^{\prime}}(E)\right|^{2}
$$

where $f$ is the reaction amplitude for producing the indicated binary fragments with channel spin $L^{\prime}, M^{\prime}$ at an angle $\theta$. This final expression differs from equation (2) only in the interpretation of $a$ and $b$, which here refer to excitation. of molecular orbitals, and through the increased subscripting and sumnations. This extra subscripting is important, though: Even when the final kinetic energy $E^{\prime}$ is sharp, the cross section must be summed over $I_{1}^{\prime}, I_{2}^{\prime}, E_{1}^{\prime}$. For any realistic experiment there will be a further summation over the range of $E^{\prime}, Z_{1}^{\prime}$ and $Z_{2}^{\prime}$ appropriate to the detectors. Thus the observed coincidence cross section involves the incoherent sum, over many quantum numbers, of the square of interfering amplitudes.

The probability for producing an electron with energy $\varepsilon$ is the joint cross section above, suitably summed, divided by the summed. nuclear cross section $\Sigma \sigma$. Consequently, the phase factor of the semi-classical theory, $\exp (-i \omega \tau)$, is replaced by $f(E-\hbar \omega) f^{*}(E)$, suitably summed, divided by $\Sigma \sigma$.

The similarity between the semi-classical and quantum-mechanical expressions for the probability of electron excitation is not without consequence. Through first order in $\omega$, the semi-classical phase factor equals $(1-i \dot{i} \tau)$ while its quantum-mechanical replacement becomes $(1-i \omega\langle\tau Q M\rangle)$ when one uses the proper 6 quantum-mechanical expression for the reaction.time, $\tau_{T M}=-i \hbar d /\left.d E \ln f(E)\right|_{E}$, and weights these by the relevant cross, sections. Tnis correspondence is quite general and does not require that any assumptions be made about the form of the reaction amplitudes. 
There are three features of this correspondence, however, which prevent us from going on to claim that the quantum-mechanical derivation, without further assumptions, justifies use of the semi-classical phase factor: (a) The quantum-mechanical time delay $\tau_{Q M}(E)$ which enters our expressions for the excitation probability is in general a complex quantity, in contrast to the real $\tau$ of the semi-classical description. For reaction amplitudes typical of low energy, light-ion reactions, the complex nature of $\tau_{Q M}(E)$ is very important and leads to observations in "time-delay" experiments 4 significantly different from those obtained using the semi-classical phase factor with real $\tau$. (b) The quantum mechanical time delay is a function of energy. For the example of light-ion resonance reactions, there can be large changes in ' $\tau_{\mathrm{QM}}$ even for changes in energy less than the total width of a level. (c) Only to first order in $\omega$ can the correlation function $f *(E-\hbar(j) f(E)$, without further restrictions, be related to ${ }{ }_{Q M}$. For arbitrary values of $\omega$, the correlation function rannot be constructed by exponentiation of $\left.\left(-i \omega{ }^{\prime} \tau_{Q M}\right\rangle\right)$ unless $f(E)$ has a very special leyendence on energy. A sufficient condition $w_{\mathrm{K}}$. Ald be that all essential

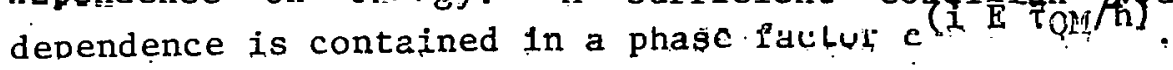

With all these points in mind we now inquire how realistic it is to anticipate oscillatory behavior in the probability for producing $\delta$-electrons or $\mathrm{K}$-shell vacancies. It appears to us that for this to occur the reaction amplitudes for energetic heavy-ion collisions must have rather special characteristics: (1) The essential $\mathrm{E}^{\text {eqergy }}$ dependence should be contained in the phase. factor suggested above $\left.e^{1\left(E^{{ }^{T}} \mathrm{QM}\right.} / \mathrm{h}\right)$, with $\tau_{2 M}$ effectively independent of energy. (2) The imaginary part of the parameter iQM should be inconsequential (i.e., the magnitude of auy partial cross sertion should not change significantly for changes in the incident energy of the order of $\pi \omega$ ). (3) Finally, the values of $\tau_{\mathrm{OM}}$ for all states significantly contributing to a measured differential cross section should be sufficiently close so that the uscillations are not washed out in taking the incoherent sum of the correlation functions to the many final states. Whether the actud nuclear amplitudes for deeply inelastic heavy-ion reactions satisfy these criteria is an open question.

\section{$\underline{\text { References }}$}

1. G. Ciocchetti and A. Molinari, Nuovo Cimento 40, 69 (1965).

2. G. Soff, J. Reinhardt, B.Müller, and W. Greiner; Phys. Rev. Lett. 43, 1981 (1979).

3. R. Anholt, Phys. Lett. 88B, 262 (1979).

4. J.S. Blair, P. Dyer, K.A. Snover, and T.A. Trainor, Phys. Rev. Lett. 41, $1712(1978)$.

5. J.S. Blair (to be published).

6. N. Austern, Direct Nuclear Reaction Theories (Wiley-Interscience, New York, 1970). 


\section{Elastic Scattering of Light Heavy Ions}

J.G. Cramer, R.M. DeVries, * D.A. Goldberg, + J.W. Watson, $X$ and M.S. Zisman + t

The understanding of elastic scattering, the simplest nuclear reaction, is central to the study of nuclear reaction mechanisms. The broad purpose of our program in elastic scattering studies of light heavy ion systems is collect enough data for the investigation of systematics, to develop global phenomenological descriptions of these data and data from other laboratories, and to investigate phenomena which are found to be deviations from such global descriptions.

We have continued the program of investigating light heavy ion elastic scattering in the region of bombarding energy where nuclear rainbow scattering influences the elastic scattering cross section. Data on the ${ }^{9} \mathrm{Be}+{ }^{28} \mathrm{Si}$ system at bombarding energies of $121.0 \mathrm{MeV}$ and $201.6 \mathrm{MeV}$, which was obtained using the LBL $88^{\circ}$ cyclotron and was reported last year, 1 has now been analyzed, and an extensive paper on the work has been accepted for publication in Phys. Rev. C. ${ }^{2}$ The data on the ${ }^{7} \mathrm{Li}+28 \mathrm{Si}$ elastic scattering at $140 \mathrm{MeV}$ obtained at LBL has been analyzed and a paper on the results is in preparation.

Since the $\alpha+{ }^{28}$ Si system had never been studied in the rainbow scattering region, we obtained new elastic scattering data on this system and the $\alpha+{ }^{27} \mathrm{Al}$ system at a bombarding energy of $142 \mathrm{MeV}$, using the University of Maryland cyclotron. These data are presently being analyzed, and should be useful in highlighting the transition from "light ion" behavior of projectiles such as ${ }^{4} \mathrm{He}$ and ${ }^{6} \mathrm{Li}$ to "heavy ion" behavior of projectiles such as $12 \mathrm{C}$ and $16^{0}$.

\section{$\underline{\text { References }}$}

* Los Alamos Scientific Laboratory, Los Alamos, NM.

$+\quad$ University of Maryland, College Park, MD.

$X \quad$ Kent State University, Kent, $\mathrm{OH}$.

+ Lawrence Berkeley Laboratory, Berkeley, CA.

1. Nuclear Physics Laboratory Annual Report, University of Washington (1979), p. 123.

2. M.S. Z1sman, J.G. Cramer, D.A. Goldberg, D.W. Watson, and R.M.DeVries, Phys. Rev. C. (in press).

11. Total Reaction Cross Section Measurements at 35 to $200 \mathrm{MeV} / \mathrm{A}$

J.G. Cramer, R.M. DeVries*, N.J. DiGiacomo*, C.R. Gruhn ${ }^{+}$, R. Loveman, J.C. Peng*, J. Sunier*, and H.E. Wiemen ${ }^{+}$

The total cross section for the nucleon-nucleon system becomes progressively smaller with energy in this energy region, presumably because of hard-core effects and the fact that the $S$-wave phase shift changes sign in this 
region. The result is that reaction cross sections for the nucleus + nucleus system should also be decreased and the very strong absorption which is a predominant characteristic of heavy ion reactions at lower energies should be reduced.

It is important to verify experimentally that such a reduction in the total reaction cross section does, in fact, exist, for if it does, the "transparency" which it gives to elastic scatter1ng at energies near $100 \mathrm{MeV} / \mathrm{A}$ will permit more detailed investigation of nuclear processes and more sensitivity to new and "exotic" phenomena than would be the case in an energy region dominated by strong absorption. As a first step to investigating the behavior of the reaction cross section in this energy region, a body of light ion ( $p, d, \alpha)$ elastic scattering data has been assembled and the optical model parameters from the analyses of these data used to infer a set of total reaction cross sprtions. 1 These inferred total reaction cross sections are found: (1) to be in good agreement with the tew measured tulal leaction crooo eactions available for these systems; (2) to be in good agreement with simple clauber-model. calculations ${ }^{2}$ based on measured electron-scattering distributions for the target and projectile and on measured nucleon-nucleon total cross sectionc; and (3) to show a clear tendency toward a significant decrease in the total reaction cross section in the region where the nucleon-nucleon total cross section becomes small. The next step in this program is to verify by direct experimental measurement that the expected reduction in reaction cross section is present.

In the past year this program has gone through a development phase, using the ${ }^{3}$ He beam of the LBL 88" cyclotron, and has had one beam-line development run and two data runs on the LBL Bevalac, all using the "1ocal" 4 He beam. The first data-collection run, which occurred in December, 1979, was successful in obtaining measured values for the total reaction cross section for $\alpha+C$ at 65 $\mathrm{MeV} / \mathrm{A}(\mathrm{cm})$ to about $8 \%$ and for $\alpha+\mathrm{Pb}$ at $85 \mathrm{MeV} / \mathrm{A}(\mathrm{cm})$ to about $13 \%$. The second $r$ un, which was in March, 1980, collected data for $135 \mathrm{MeV} / \mathrm{A} \propto$ on targets of carbon, nickel, zirconium, and lead. These data are presently being analyzed. However, preliminary analysis indicates that the total reaction cross section in this energy region is indeed considerably smaller than "geometric", and in fact appears to be significantly swaller than the Glauber-model predictions described above.

\section{References}

* Los Alamos Scientific Laboratory, Los Alamos, NM.

$+\quad$ Lawrence Berkeley Laboratory, Berkeley, CA.

1. R.M. DeVries and J.C. Peng, Phys. Rev. Lett. 43, 1373 (1979).

2. R.M. DeVries and J.C. Peag, Phys. Rev. Lett. (to be published). 
12. The Elastic Scattering Cross Section of $16_{\mathrm{O}}+{ }^{40} \mathrm{Ca}$ and the Proximity Potential

S. Gil and R. Vandenbosch

The motivation of this study was to investigate a concern, expressed in a recent report, 1 that the proximity potential ${ }^{2}$ was too diffuse to reproduce the elastic scattering for $160+40^{\mathrm{Ca}}$.

For this purpose we have modified the code HOP II 3 to include the proximity potential as an option for the real part of the potential. For the imaginary part we have used a Woods-Saxon-like function. The data used were obtained by Vigdor et al.

The cross section shows a great sensitivity to the radius of the real part. We have observed that the prescription suggested by Blocki et al. 2 for the calculation of the "Central Radii" (C) underestimate their value for $16_{0}$ and ${ }^{40} \mathrm{Ca}$, this is also observed by $\mathrm{N}$. Voa Sen et al. 4 for the case of $20 \mathrm{Ne}+40_{\mathrm{Ca}}$. This is not surprising as the masses involved are nearly outside the range of masses for which the prescription is expected to be valid.

For this reason we have used the value of the radii obtained from electron scattering, i.e.,

$$
C\left(4^{40} \mathrm{Ca}\right)=3.75 \mathrm{fm} \cdot \text { and } \quad C\left({ }^{16_{0}}\right)=2.61 \mathrm{fm}
$$

(Taken from Ref. 5). With such a choice the real part of the potential was held fixed.

The values of the parameter of the imaginary part were varied so as to minimize chi-square for the case of $\mathrm{E}_{\mathrm{LAB}}=55.6 \mathrm{MeV}$ data. The variation of these parameters was seen to produce changes in the differential cross section similar to the ones observed in the case of a pure Woods-Saxon potential. In Table 6.12-1. we show the values obtained for chi-square and $\sigma_{R E A C}$ for both the proximity and the Woods-Saxon real potentials, as well as the parameters used in each case.

It can be seen that the values of $\sigma_{R E A C}$ are very close for both potentials, especially if we take into account the fact that there is a spread in the values

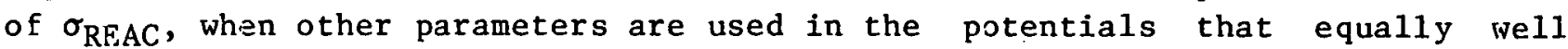
could fit, the data.

It is also instructive to see the graph of the real potential for both cases analyzed, which clearly depicts the well known fact that the elastic scattering with heavy-ions is sensitive only to the narrow part of the potential tail around the strong absorption radius (Fig. 6.12-1). 
Table 6.12-1

Woods-Saxon

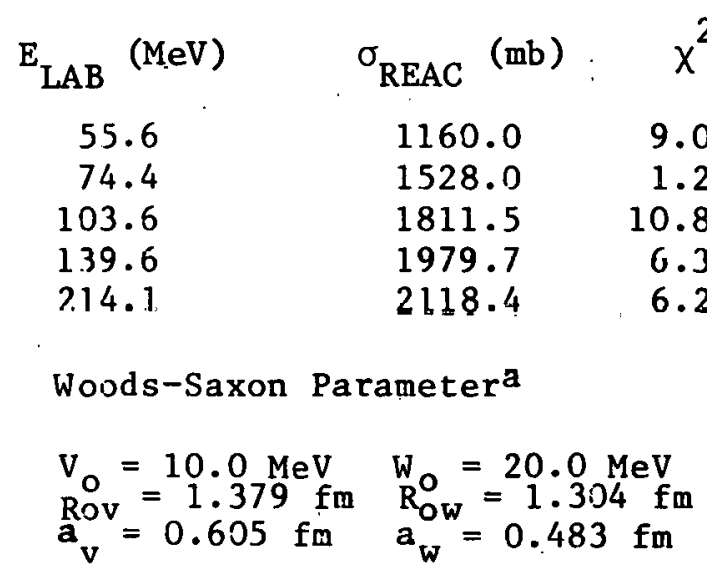

Proximity

\begin{tabular}{cr}
$\sigma_{\text {REAC }}(\mathrm{mb})$ & \multicolumn{1}{c}{$\chi^{2}$} \\
1169.3 & 10.28 \\
1531.0 & 1.67 \\
1810.0 & 14.09 \\
1970.0 & 15.70 \\
2094.0 & 52.20
\end{tabular}

Proximity ra rämeter ${ }^{a}$

$$
\begin{gathered}
C(160)=2.61 \mathrm{fm} \\
C\left(40_{C a}\right)=3.75 \mathrm{fm} \\
W=10.0 \mathrm{MeV} \\
R_{O W}=1.330 \mathrm{fm} \\
a_{w}=0.550 \mathrm{fm}
\end{gathered}
$$

a The radius parameters are to be multiplied by $\left(A_{1}^{1 / 3}+A_{2}^{1 / 3}\right)$. The Coulomb radius parameter was held fixed for all potentials at $1.25 \mathrm{fm}$. 


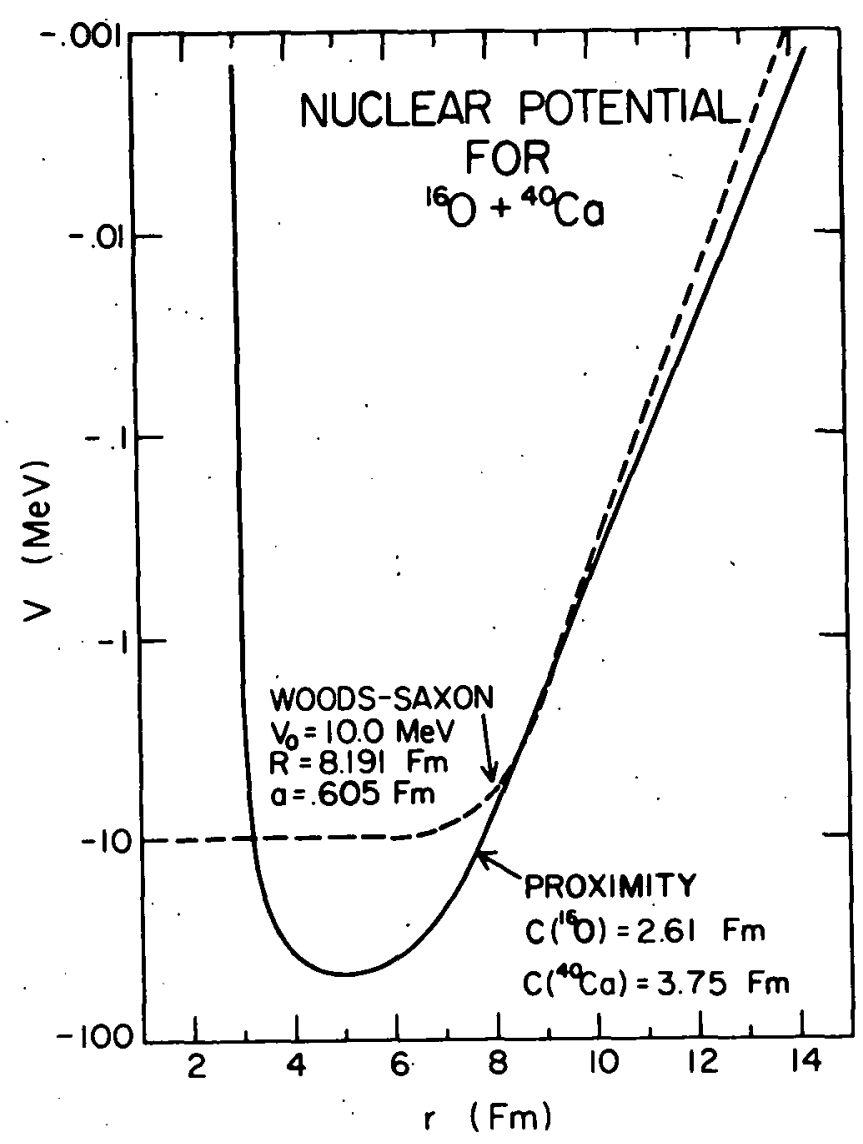

Fig. 6.12-1: Comparison of the real potential used in our calculation with the Woods-Saxon potential of Vigdor et al. The parameters are given in Table 6.12-1.

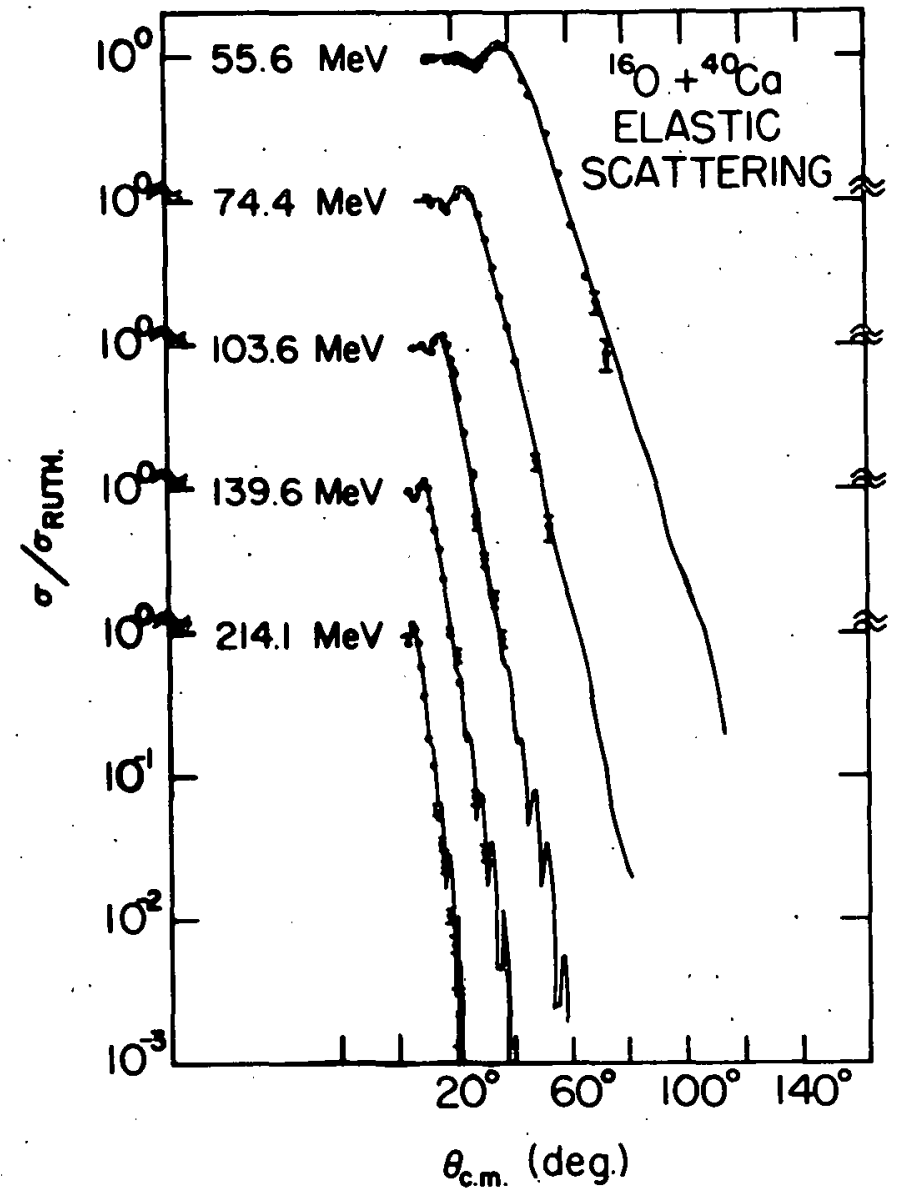

Fig. 6.12-2: Elastic scattering angular distributions for $160+{ }^{4} 0_{\mathrm{Ca}}$ compared with the optical model calculation using the proximity potential with +parameter given in Table 6.12-1. The experimental data were measured by Vigdor et al. (Ref. 1). 
Finally, it is interesting to see that the fits obtained using this proximity potential (Fig. 6.12-2) are nearly as good descriptions as the Woods-Saxon ones, with the difference that in the former case there are no adjustable parameters in the real part.

\section{$\underline{\text { References }}$}

1. S.E. Vigdor, D.G. Kovar, P. Sperr, J. Mahoney, A. Menchaca-Rocha, C. Olmer, and M.S. Zisman, Phys. Rev. 20, 2147 (19979)).

2. J. Blocki, J. Randrup, W.J. Swiatecki, and C.F. Tsang, Ann. of Phys. (NY), 105, 427 (1977).

3. Nuslear Physics Laboratory Annual Report, University of Washington (1978), p. 133 .

4. Nguyen Van Sen, R. Darves-Blane, J.C. Gondrand, and F. Merchez, Phys. Rev. C20; 969 (1979).

3. C.W. de Jager, H. deVries, and C. deVries, Atomic and Nuclear Data Tables, 14, 479 (1974).

13. Non-Resonant Behavior of the Yield of the ${ }^{28} \mathrm{Si} 2^{+}$state in ${ }^{12} \mathrm{C}+{ }^{28} \mathrm{Si}$

A.J. Lazzarini, K. Lesko, D.-K. Lock, V. Metag, and R. Vandenbosch

Recent studies of back-angle elastic and inelastic scattering excitation functions for the $12 \mathrm{C}+28 \mathrm{Si}$ reaction have revealed energy-dependent gross siructure with a periodicity of about $3 \mathrm{MeV}$. This structure is reminiscent of the behavior observed in the lighter systems, ${ }^{12} \mathrm{C}+{ }^{12} \mathrm{C}$ and $12 \mathrm{C}+16_{\mathrm{O}}$ but was not expected for such heavy systems. Various explanations for the structure have been offered. They can roughly be divided into two classes; those identifying the structures as resonances related to the ion-ion potential or band crossings, and those attributing the structure in the differential cross section to interference effects among different surface partial waves. These two classes of explanations should be distinguishable by measuring the angle-integrated strength for the inelastic scattering.

We have used a gamma ray technique to measure the angle-integrated cross section for producing the first excited ( $\mathrm{J}^{\pi}=2^{+}, \mathrm{E}=1.78 \mathrm{MeV}$ ) state of ${ }^{28} \mathrm{Si}$. A $113 \mu \mathrm{g} / \mathrm{cm}^{2}$ target mounted on a thick Ta backing was bombarded by ${ }^{12} \mathrm{C}$ ions. Two $\mathrm{Ge}(\mathrm{Li})$ detectors were placed at $90^{\circ}$ and $144^{\circ}$ with. respect to the beam. The ratio of the 1440 cross section to the $90^{\circ}$ cross section was found to be independent of bombarding energy. 'l'he angle integrated cross section was c......ed by assuming a distribution of the form a $+b \cos ^{2} \theta+c \cos ^{4} \theta$ aud deternining the coefficients from the fit to a 3-point angular distribution. This leads to an angle-integrated cross section which is $(1.07 \times 4 \pi)$ times larger than the $90^{\circ}$ differential cross section. 
The gamma ray determination of the total inelastic cross section to the $2^{+}$ state of ${ }^{28} \mathrm{Si}$ varies smoothly with energy and does not exhibit the structure seen in the inelastic differential cross section at back angles. Thus there is no structure in the energy dependence of the total inelastic scattering strength. This behavior, which is different from that exhibited by lighter systems such as ${ }^{12} \mathrm{C}+{ }^{12} \mathrm{C}$, is inconsistent with a resonance interpretation. It is more consistent with an interpretation such as that given by Kubono et al. 3 who interpret the $180^{\circ}$ structure as arising from an interference effect due to an effective potential which is parity dependent. They were led to this conclusion by an examination of the elastic scattering excitation function at $90^{\circ}$, which was found to be out of phase with that at $180^{\circ}$.

\section{References}

1. J. Barrette, M.J. LeVine, P. Braun-Munzinger, G.M. Berkowitz,. M. Gai, J.W. Harris, and C.M. Jachcinski, Phys. Rev. Lett. 40, 445 (1978).

2. M.R. Clover, R.M. DeVries, R. Ost, N.J.A. Rust, R.N. Cherry, Jr., and H.E. Gove, Phys. Rev. Lett. 40, 1008 (1978).

3. S. Kubono, P.D. Bond, D. Horn, and C.E. Thorn, Phys. Lett. $84 \mathrm{~B}, 408$ (1979). 


\section{RESEARCH BY OUTSIDE USERS}

\section{Alpha-N Yield Neutron Measurements of Importance to Reactors*}

P.J. Grant, ${ }^{+}$D.L. Johnson, ${ }^{++}$and G.L. Woodruff ${ }^{+}$

The neutron yields produced by $(\alpha, n)$ reactions of importance to reactors are to be measured with incident alpha energies ranging from $4 \mathrm{MeV}$ to $8 \mathrm{MeV}$. Initial experiments will focus on $18_{0}$ targets followed by others as interests dictate.

A large $(\sim 1.5 \mathrm{~m})$ graphite assembly has been constructed as a cylindrical. "long counter" on the zero degree beam line. I 'lhe assembly cuntuilis an array of ten $3_{\mathrm{He}}$ detectors perpendicular to and at varying angles with respect to the inroming $\alpha$-particle beam. The target is at the center of the assembly and is surrounded by a $15.2 \mathrm{~cm}$ diameter spherical shell of either he or Fe in order to flatten the detection efficiency at high energies.

A preliminary set of results for 180 is shown in Fig. 7.1-1 together with those reported by Bair and Gomez del Campo ${ }^{2}$ and West and Sherwood. ${ }^{3}$ The assembly has not yet been completely calibrated; therefore the results in Fig. 7.1-1 are normalized to the Bair volume at $7 \mathrm{MeV}$. The relative values appear. to be in fairly good agreement.

\section{References}

* Work supported by Department of Energy Contract EY-76-S-2225 TA45.

+ Department of Nuclear Engineering, University of Washington.

Hestinghouse, Hanford.

1. Nuclear Physics Laboratory Annual Keport, Untversity of Washington pp. 131,132 .

2. J.K. Bair and J. Gomez del Campo, Nuc. Sci. Eng. 21, 18 (1979).

3. D. West and A.C. Sherwood, Proceedings of Int1. Conf. on Neutron Physics and Nuclear Data for Reactors and Other Applied Purposes, Harwell, U.K. (Sept. 1978).

Fig. 7.1-1: $18_{O}(\alpha, n)$ yields vs. alpha energy.

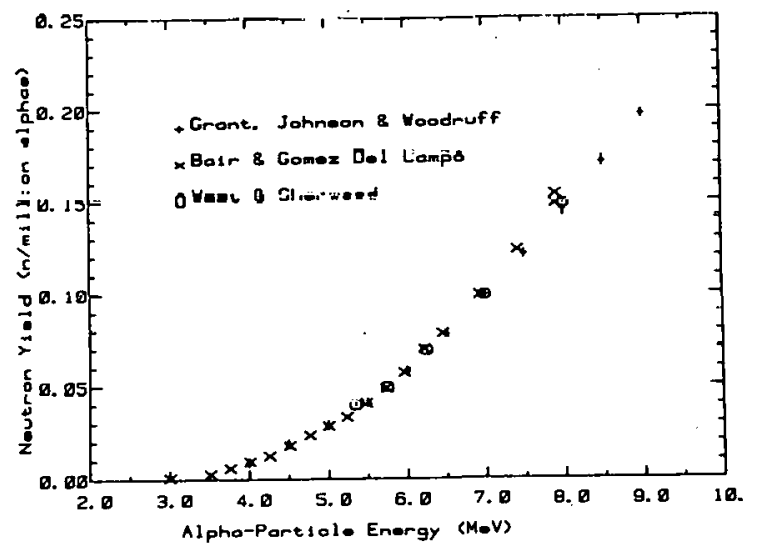


2. Fast Neutron Beam Radiation Therapy Clinical Program.

\section{G.E. Laramore ${ }^{+}$}

Early in 1979 fast neutron beam radiotherapy clinical studies were reinstituted using the University of Washington cyclotron. The majority of patients were treated according to randomized prospective protocols developed here and at other neutron radiotherapy institutions. In these studies patients were randomly assigned either neutrons alone, neutrons and photons as part of a mixed beam regime (neutrons twice a week and photons three times a week), or photons alone. A total of 109 patients was entered on these studies with a total of 65 patients receiving neutrons as all or a part of their treatment. . In addition 38 other patients received non-protocol neutron irradiation either to obtain information for future protocol development or in situations where it was felt that the benefits of neutron radiation outweighed any potential side effects. Including data from the other institutions participating in neutron radiotherapy, the following information has been obtained for various tumor sites.

1) Squamous Ce11 Carcinomas of the Head and Neck (Inoperable)

This study randomizes patients between the mixed beam regime and photon radiation alone. Preliminary results indicate about a 15-20\% greater 1ocal control rate for the mixed beam group. In fact, patients are now surviving long enough to develop problems with distant metastases which will lead to new study protocols involving chemotherapy as well as neutron radiation.

2) Malignant Glionas

In this study patients initially receive 5000 rady whole brain photon irradiation and then are randomized to either a 1500 rady photon boost or a $480 \mathrm{rad}_{\mathrm{n}}$ neutron boost to the primary tumor volume. The uncorrected data show a 33-week median survival for the group receiving the photon boost and a 39-week median survival for the group receiving the neutron boost. In the subgroup of patients surviving more than one year, 3 come from the photon boost group and 7 come from the neutron boost group, which may indicate substantially different tails on the respective survival curves.

3) Advanced Prostate Cancer

In this study patients are randomized to either mixed beam or photon irradiation. Follow-up time is short but thus far local control has been achieved in $75 \%$ of the patients treated with photons alone and in $94 \%$ of the patients treated with mixed beam regime.

4) Inoperable Non-0at Cell Lung Cancer

In this study patients are randomized among three treatment arms: neutrons alone, mixed beam or photons alone. It appears that the group treated with neutrons alone shows a significantly more rapid and greater degree of tumor regression than patients in the other treatment arms. 
Follow-up time is too short to say anything about any difference in patient survival.

Other tumors under study include carcinomas of the esophagus, uterine cervix, bladder, salivary glands, and malignant melanomas.

In the past year a significant problem has been loss of treatment time because of cyclotron malfunction. This has caused considerable patient anxiety as well as several violations of the planned protocol treatments. We have shifted treatment days to allow Monday mornings for maintenance and this may alleviate this problem during the coming year.

\section{$\underline{\text { References }}$}

* Supported by NCI Grant No. CA-12441.

+ Department of Radiation Oncology, University of Washington.

3. Total Body Calcium by Neutron Activation

D.J. Baylink, ${ }^{+}$C.H. Chesnut, ${ }^{+}$T.K. Lewellen, ${ }^{+}$R. Murano, ${ }^{+}$and

W.B. $\mathrm{NeI}^{+}$

The Division of Nuclear Medicine is continuing its studies of bone wasting disease. 1 Total body calcium is measured by neutron activation and whole-body counting. The cyclotron is used as the neutron source, and the $3.1 \mathrm{MeV}$ gamma ray of 8.7 minute ${ }^{49} \mathrm{Ca}$ is counted. 2

The drug Winstral (Winthrop Laboratories) has been tested for the rnntrol ot post-menopausai osteoporosis. Twenty-three (23) treated individuals showed an average increase of $4.2 \%$ in total body calcium over 27 months, distributed with a standard deviation of $6.9 \%$. Nineteen (19) control individuals showed no change in total body calcium. While a minority of treated individuals showed no improvement, the average increase of $4.2 \%$ is highly significant, the standard error of the mean being $6.9 \% / 23=1.4 \%$. Both groups were maintained on high calcium diets.

A similar study using the hormone calcitonin showed an increase of $2.2 \%$ over 27 months compared to a decrease of $1.6 \%$ for the control group. The difference from the other control group, if it is significant, may be due to the dietary calcium supplement.

A pilot study of the drug Dichloromethane Diphosphonate (Proctor \& Gamble) was undertaken in cooperation with $R . R$. Recker of Creighton Universily. The results on this small group of patients (16) were positive enough to justify a more extensive investigation with a larger number of patients. 


\section{References}

+ Division of Nuclear Medicine, University of Washington.

1. J.L. Marx, "Osteoporosis: New Help for thinning Bones," Science

2. Nuclear Physics Laboratory Annual Reports, 1968-1978.

4. ${ }^{81} \mathrm{Kr}^{\mathrm{m}}$ Production for Respiratory Physiology

M.M. 'Graham, ${ }^{+}$H.I. Mode11, ${ }^{++}$and R. Murano ${ }^{+}$

$4.7 \mathrm{hr}{ }^{81} \mathrm{Rb}$ is produced by the $\alpha$-bombardment of $\mathrm{NaBr}$ at the University of Washington cyclotron.1 The target is dissolved in water and adsorbed on a column. Air or nitrogen flowing through the column carries 13 second ${ }^{8} \mathrm{Kr}_{\mathrm{K}}^{\mathrm{m}}$ into a respirator from which an experimental animal breathes.

A gamma camera is used to create an image of the animal's lungs. Experiments to date have shown that the total lung activity is proportional to the ventilation, or fractional volume exchanged per unit time.

\section{$\underline{\text { References }}$}

+ Division of Nuclear Medicine, University of Washington.

+ Department of Physiology and Biophysics, University of Washington.

1. Nuclear Physics Laboratory Annual Report, University of Washington (1979), p. 128 .

5. Cyclotron Production of $105 \mathrm{Ag}$

J. Arzigian, ${ }^{+}$G. Depasquali, ${ }^{+}$and D. Lazarus ${ }^{+}$

$\mathrm{Ag}_{4} \mathrm{RbI}_{5}$ is an ionic material with unusually large values for the diffusion coefficient of silver ions near room temperature ( $\left.D=10^{-5} \mathrm{~cm} / \mathrm{sec}\right) . \mathrm{Ag}_{4} \mathrm{RbI}_{5}$ and other "superionic" materials show great promise as materials for high energy density solid state batteries. To understand the transport mechanism involved, we are making isotope effect measurements for diffusion using commercially available $110 \mathrm{~m}_{\mathrm{Ag}}$ and $105_{\mathrm{Ag}}$ produced in the reaction $103_{\mathrm{Rh}(\alpha, 2 \mathrm{n})}{ }^{105} \mathrm{Ag}$ with cyclotron alpha particles.

\section{References}

$+\quad$ University of Illinois, Urbana-Champaign, IL. 


\section{C.H. Henager, ${ }^{+}$R.G. Stang, ${ }^{+}$E.P. Simonen,* J.L. Brimhall*}

Materials placed under stress and subjected to the elevated neutron fluxes of both breeder and fusion reactors exhibit a form of accelerated deformation termed irradiation creep. Due to the difficulty, cost and time required in making precise in-reactor creep measurements, there is a world-wide interest in simulating in-reactor creep by bombarding materials of interest with energetic light ions under well controlled conditions. Such experiments enhance our understanding of the operating creep mechanisms and permit screening of potential reactor alloys. The Radiation Effects on Metals program supported by the Division of Basic Energy Sciences, Department of Energy at Battelle, Pacific Northwest Laboratories is conducting an irradiation creep experiment using the tandem Van de Graaff accelerator at the Nuclear Physics Laboratory.1

Early experiments compared the creep response of high purity $\mathrm{Ni}$ under conditions of continuous and cyclic irradiation with $17 \mathrm{MeV}$ deuterons. The cyclic irradiation approximated the expected duty cycle of a tokamak fusion power plant. The significant result of this series of experiments was that cyclic irradiation creep is greater than that expected for continuous irradiation. 2,3

At the present time a series of experiments to determine the stress dependency of irradiation creep in $\mathrm{Ni}$ is near completion. Continuous irradiations with $17 \mathrm{MeV}$ deuterons have been performed at $200^{\circ} \mathrm{C}$ with stresses ranging from 250 to $132 \mathrm{MPa}$. To date, 9 irradiations at 6 different stress levels have been completed. Preliminary analysis using earlier results indicates that the stress exponent for the continuous irradiations is about two.4 Including this later data gives a stress exponent of about 1.5 . Theoretical modelling of the irradiation creep process is being pursued at PNL. 5

Several significant changes in experimental instrumentation have been made during the past year. An in-chamber movable beam stop has been added to facilitate heam tuning without irradiating the specimen. A quadrant plate assembly was installed just upstream of this new beam stop for tuning and steering purposes. Using the quadrant output, the accelerator operator can determine beam location relative to the creep specimen. The most significant change was the addition of a PDP-11/03 computer and a NEFF System 62024 channel $\mathrm{A} / \mathrm{D}$ converter as the new data acquisition system. 6 This new system has greatly increased data accuracy and has sufficient flexibility to accommodate a greater degree of sophistication in the future, e.g., computer based parameter control.

\section{References}

+ Department of Metallurgical, Mining, and Ceramic Engineering, University of Washington, and Pacific Northwest Laboratories, Richland, Washington.

+ Department of Metallurgical, Mining, and Ceramic Engineering, University of Washington.

* Pacific Northwest Laboratories, Richland, Washington. 
1. P.I. Hendrick, Nucl. Instr. and Meth., 161, 345 (1979).

2. E.P. Simonen and P.L. Hendrick, J. Nucl. Mat., $85 \& 86,873$ (1979).

3. E.P. Simonen, J. Nucl. Mat., 1980 (accepted for putlication).

4. C.H. Henager, J.L. Brimhall, E.P. Simonen, J. Nucl. Mat., 1980 (accepted for publication).

5. E.P. Simonen, C.H. Henager, abstr. accepted for presentation at 10th International Symposium on Effects of Radiation on Materials, ASTM, Savannah, GA, June 3-5, 1980.

6. P.L. Hendrick, T.J. Whitaker, PNL-3066, UC-25, July 1979 (available from NTIS ).

7. A Comparison of the Therapeutic Effectiveness of BCNI Administered Concurrently with Neutron or Photon Exposures and with BCNU Administered One Day Following Neutron or Photon Exposures to a Rat Brain Tumor Model

J.P. Geraci ${ }^{+}$and A.M. Spence ${ }^{++}$

Between 1973 and 1977 the radiation oncologists at the University of Washington treated 22 cases of high grade astrocytic gliomas with whole-brain fast neutron beam irradiation. Patients receiving neutron treatment did have a slightly longer mean survival time than an historical control group of photon-treated patients at the University of Washington. Of particular interest were the neuropathological analyses of 15 patients who came to autopsy. The autopsies revealed that the destructive effect of neutrons on glioblastoma was substantial as compared to conventional photon irradiation. However, diffused gliosis and white matter demyelinization were found in regions far removed from the tumor which were in excess of those found with equivalent doses of photon exposure. These results suggest that a possible refinement in neutron therapy using lower neutron doses to avoid excessive normal tissue damage combined with non-toxic levels of chemotherapeutic agents may still achieve an optimum tumor response. To ascertain whether this is so we have studied the effects of cyclotron neutrons, ${ }^{137} \mathrm{Cs}$ gamma rays, and $\mathrm{BCNU}$ (the leading chemotherapeutic agent for the treatment of human gliomas) or combinations thereof on a transplantable ethylnitrosourea-induced rat astrocytoma.

The effects of administering $B C N U$ either one hour before or one day after irradiation are shown in Fig. 7.7-1. For animals treated with BCNU one day after photon irradiation, the results show approximately a constant $50 \%$ displacement of the percent survival curve as compared to the response of animals receiving photons alone, except at the highest radiation dose used. Since a $50 \%$ increase in survival time is also observed in animals receiving no radiation, these results indicated that the effects of $B C N U$ and radiation are additive when drug is administered one day after radiotherapy. In contrast, when $B C N U$ therapy precedes photon irradiation by one hour the drugs appear to yield a greater than additive effect in enhancing the survival of tumor bearing animals. At high radiation doses coupled with BCNU therapy (2000 rad) there is a significant reduction in survival of animals, presumably due to the combined insults of radiation, drug, and tumor growth. 


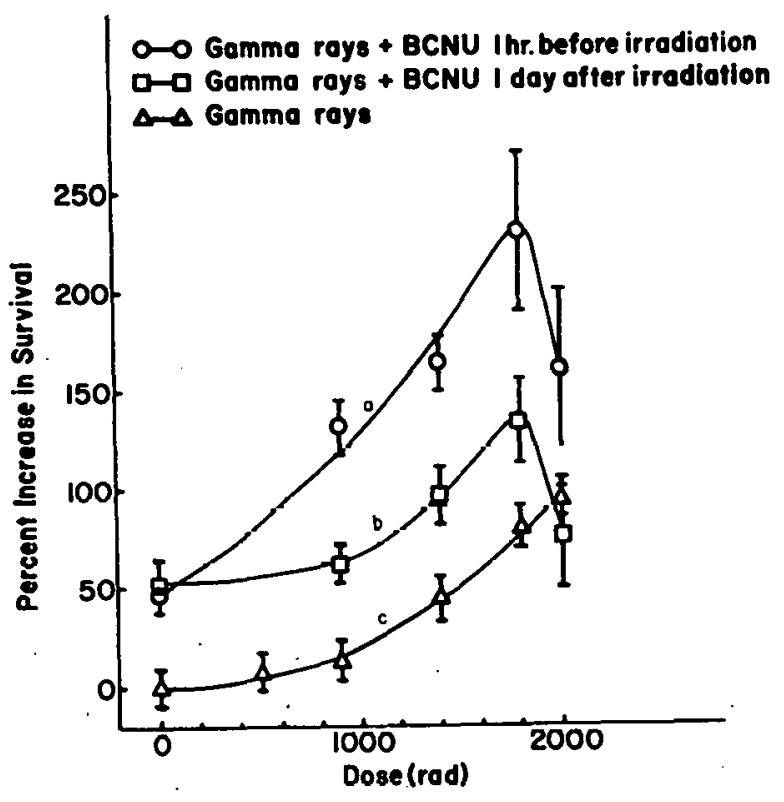

Fig. 7.7-1: Effects of administering BCNU before or after gamma irradiation

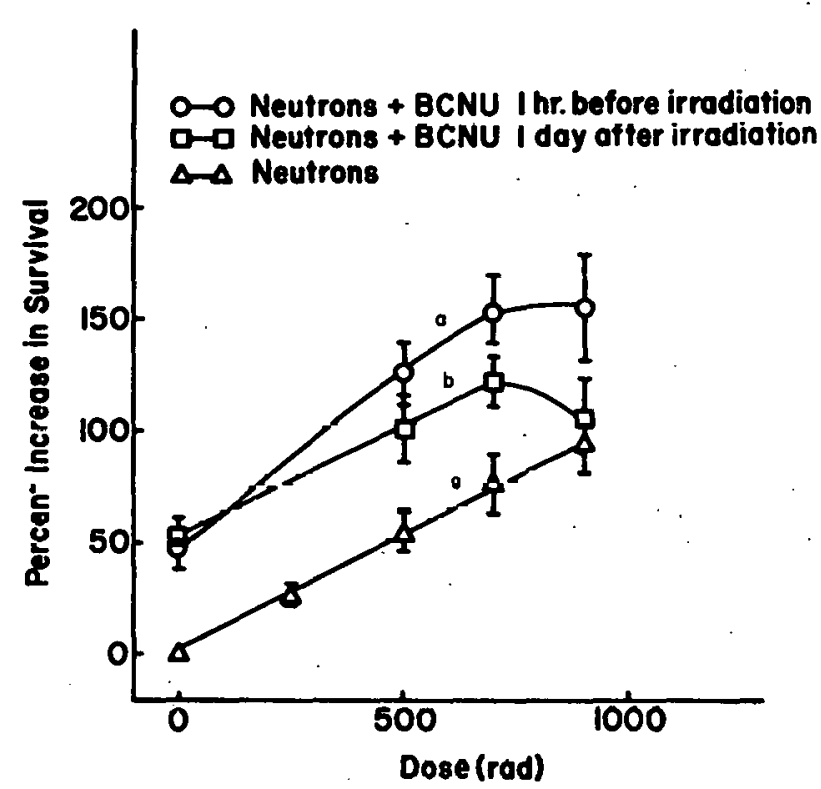

Fig. 7.7-2: Effects of administering BCNU before or after neutron irradiation

The results of a comparable experiment for neutron irradiated animals are shown in Fig. 7.7-2. These results again show a parallel dose response relationship between animals receiving neutrons alone and neutrons plus BCNI one day after exposure. The increase in survival by addition of $B C N U$ to neutron irradiated tumor bearing animals is approximately $50 \%$ for all neutron doses, which is similar to that observed for the photon tested animals. Also similar to photon experiments is that when BCNU is administered one hour before irradiation there appears to be a greater than additive effect in prolonging the survival of these animals. However, this super-additive effect appears to be less than that observed for photon treated animals. As was the case with photon treated animals, large neutron doses (1000 rad) coupled with BCNU therapy resulted in reduced survival time as compared to animals receiving lower radiation dooec.

\section{References}

+ Radiological Sciences, Department of Environmental Health, School of Public Public Health and Community Medicine.

+ Department of Medicine (Neurology), School of Medicine. 
8. Calibration of the Proton Registration Properties of CR-39 Polymer

F.H. Ruddy, ${ }^{+}$C.C. Preston, ${ }^{+}$R. Gold, ${ }^{+}$E.V. Benton ${ }^{++}$and J.H. Roberts ${ }^{++}$

In support of materials development for the Magnetic Fusion Energy Program, the United States Department of Energy is constructing an intense neutron source known as the Fusion Materials Irradiation Test (FMIT) Facility.1 The FMIT Facility will generate high energy neutrons for the systematic study, evaluation, and development of fusion reactor materials. A prototype linear accelerator will provide a high current deuteron beam ( $100 \mathrm{ma}, 20$ and $35 \mathrm{MeV}$ ) that will impinge on a target of flowing liquid lithium, producing neutrons through the $\operatorname{Li}(\mathrm{d}, \mathrm{n})$ reaction. Flux intensities of $10^{15}$ neutrons $/\left(\mathrm{cm}^{2}-\mathrm{sec}\right)$ with a most probable energy of $14 \mathrm{MeV}$ are anticipated. Current plans call for beam on target in 1984 .

No irradiation facility yet built approximates the irradiation environment planned in FMIT. Full exploitation of this unique facility requires the development and testing of dosimetry techniques applicable in intense high energy neutron fields. One of the potential methods being developed is the use of solid state track recorders.

Since the discovery 2,3 of the track recording properties of CR-39 polymer in 1978, this plastic has been shown to have a number of properties advantageous to neutron dosimetry applications. Among the most important of these are excellent optical quality (even after extensive chemical etching), with resistance to $\beta-\gamma$ radiation, 4 and sensitivity to protons over a wide energy range.5 Calibration, experiments carried out using University of Washington proton beams have shown that protons will register as tracks in the energy range from 0.2-18 MeV.6 Furthermore, the diameters of normally incident tracks have been shown to vary smoothly with energy allowing the use of this material for proton spectroscopy (see Fig. 7.8-1). Plans for neutron dosimetry involve the use of CR-39 for neutron induced proton recoil spectrometry,7,8 relying on the $H(n, p)$ cross section which is accurately known over the entire energy range of FMIT $(0-40 \mathrm{MeV})$.

The proton registration efficiency of $\mathrm{CR}-39$ polymer as a function of angle of incidence has recently been calibrated at two energies and present plans call for continued angular response calibrations as well as extension of the track diameter versus proton energy curve to higher energies.

\section{References}

$+\quad$ Hanford Engineering Development Laboratory.

+ University of San Francisco.

H Macalester College.

1. E.W. Pottmeyer, Jr. "Construction of a High Intensity Deuteron Linac with an Energy of $35 \mathrm{MeV}$ and an Intensity of $100 \mathrm{~mA} \mathrm{CW}, "$ HEDL-SA 1652, Sixth Al1-Union National Conference on Particle Accelerators, Dubna (1978)': E.W. Pottmeyer, Jr. "The Fusion Materials Irradiation Test Facility at Hanford," HEDL-SA 1734 (1979): 
2. B.G. Cartwright, E.K. Shirk, and P.B. Price, "CR-39: A Nuclear Track Recording Polymer of Unique Sensitivity Resolution," Nucl. Inst. and Methods, 153, 457 (1978).

3. R.M. Cassou and E.V. Benton, "Properties and Applications of CR-39 Polymeric Nuclear Track Detector," Nuclear Track Detection, 2, 73 (1978).

4. R. Gold, F.H. Ruddy, E.P. Lippincott, W.N. McElroy and J.H. Roberts, "Spent Thermal Reactor Fuel Assembly Characterization with Solid State Track Recorders," HEDL-TME 78-89 (1979); R. Gold, F.H. Ruddy, E.P. Lippincott, W.N. McElroy and J.H. Roberts, "Neutron Dosimetry Characterization of Spent Thermal Reactor Fuel Assemblies," Trans. Am. Nuc. Soc. 32, 634 (1979).

5. E.V. Benton, C.C. Preston, F.H. Ruddy, R. Gold, and. J.H. Roberts, "Proton and Alpha Particle Response Characteristics of CR-39 Polymer for Reactor and Dosimetry Applications," in Proceedings of the Tenth International Conference on Solid State Nuclear Track Detectors, Lyons (1979).

6. F.H. Ruddy, C.C. Preston, R. Gold, E.V. Benton, and J.H. Roberts, "CR-39 Polymer, A Promising New Solid State Track Recorder for High Energy Neutron Applications," Second Symposium on Neutron Cross Sections from 10-50 MeV, Brookhaven (1980).

7. R. Gold, J.H. Roberts, and F.H. Ruddy, "Advanced in Solid State Track Track Recorder Techniques for Dosimetry and Radiation Damage Measurements," Proceedings of the Third ASTM-EURATOM International Symposium on Reactor Dosimetry, Ispra, Italy (1979).

8. R. Gold, F.H. Ruddy, C.C. Preston, J.H. Roberts, and W.N. McElroy, "Neutron Camera for Fusion Diagnostics," Third APS Topical Conference on High Temperature Plasma Diagnostics, Los Angeles (1980).

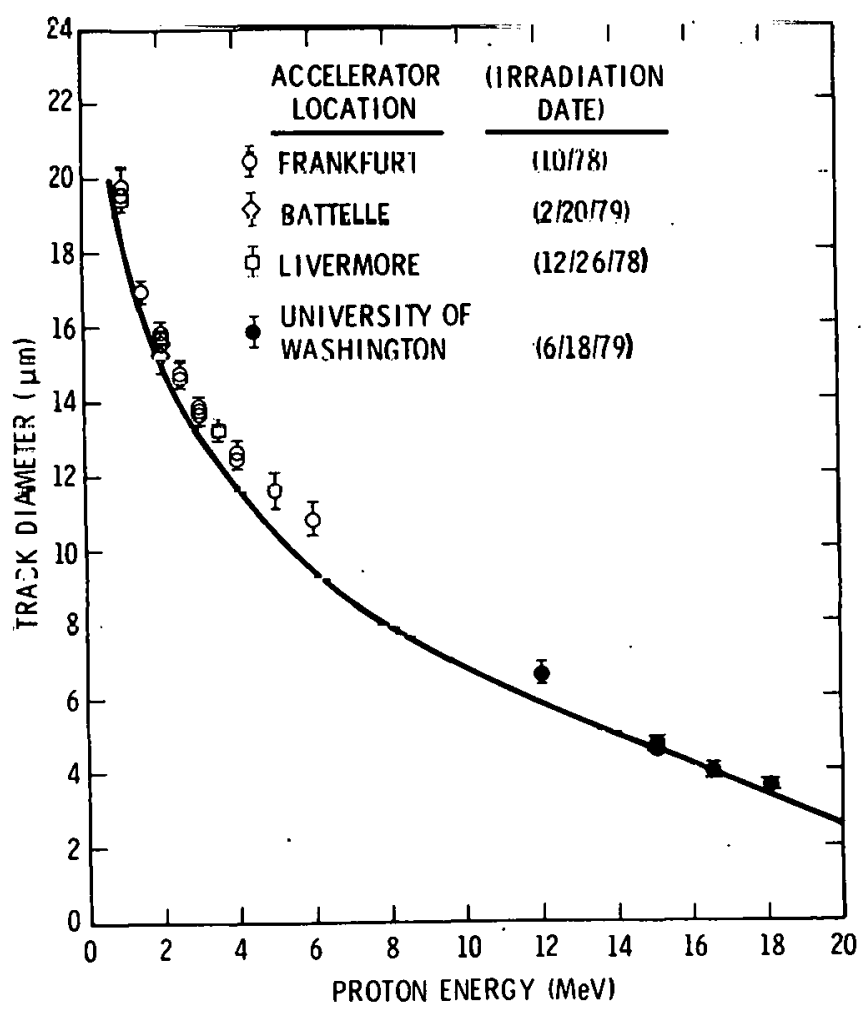


L.E. Antonuk, + and P. Kitching ${ }^{+}$

A $50.88 \mathrm{mg} / \mathrm{cm}^{2}{ }^{40} \mathrm{Ca}$ target used in a previous ${ }^{40} \mathrm{Ca}(\mathrm{p}, 2 \mathrm{p}) 200 \mathrm{MeV}$ experiment at TRIUMF was suspected to be heavily contaminated by oxygen. The analysis of these data required a knowledge of the amount of oxygen relative to calcium in this target. To measure this quantity, a singles experiment was performed in the large scattering chamber using $18 \mathrm{MeV}$ protons. Elastically and inelastically. scattered protons were measured with a solid state silicon detector capable of $50 \mathrm{keV}$ resolution. Besides the $\mathrm{Ca}$ target, energy spectra were taken for a $0.400 \mathrm{mg} / \mathrm{cm}^{2}{ }^{12} \mathrm{Ca}$ target and a $1.8 \mathrm{mg} / \mathrm{cm}^{2} \mathrm{MgO}$ target to assist in peak identification. The detector angle was chosen as $110^{\circ}$ in order to maximize the separation of the $16_{0}$ elastic peak $(15.174 \mathrm{MeV})$ from the elastic $(16.811 \mathrm{MeV})$ and first excited state $(13.572 \mathrm{MeV})$ of $40_{\mathrm{Ca}}$. The ratio of. $16_{0}$ to ${ }^{40} \mathrm{Ca}$ atoms in the $\mathrm{Ca}$ target was determined to be 1/1:9. In addition, the amounts of ${ }^{14} \mathrm{~N}$ and ${ }^{12} \mathrm{C}$ relative to ${ }^{40} \mathrm{Ca}$ were determined to be less than $1 / 14$ and $1 / 7.4$, respectively.

\section{References}

$+\quad$ Nuclear Research Centre, University of Alberta, Edmonton, Alberta.

10. Pulsed Radioluminescence Studies*

M.L. West ${ }^{+}$and J.H. Miller ${ }^{+}$

Pulsed radioluminescence techniques were used to investigate the fluorescence response of dilute solutions of benzene in cyclohexane for proton and alpha particle excitation. For both protons and alpha particle excitations, the initial rate of decay of benzene fluorescence is energy dependent and is always greater than the rate of decay observed with ultra violet excitation. A diffusion kilutlc model based on intra track quenching from radicals created along individual particle tracks has been formulated to explain these observations. The intra track quenching is observed to be proportional to the mean stopping power of the incident ion. However, for protons and alpha particles of the same mean stopping power, the amount of quenching for alpha particles is about 60 per cent of that observed for protons. These results establish a radiation quality effect in the characterization of radioluminescence that is consistent with our model predictions.

\section{Rcferences}

* This work is performed under United States Department of Energy Contract DE-AC06-76RLO.

$+\quad$ Battelle Pacific Northwest Laboratory, Richland, Washington 99352. 


\section{ACCELERATORS AND ION SOURCES}

1. Accelerator Radiochronology

G.W. Farwe11, P.M. Grootes, ${ }^{+}$W.A. Saunders, F.H. Schmidt, and M. -Y.B. Tsang

The use of the tandem Van de Graaff as an ultrasensitive mass spectrometer for the measurement of minute isotopic ratios, with particular attention to applications in radiochronology (such as ${ }^{14} \mathrm{C}$ dating), was begun in 1977 and has continued at a somewhat accelerated pace.

During 1979 we have concentrated on the following problems:

1) Improvement in general stability of the tandem accelerator and the sputter-type ion source.

2) Study of the background of unwanted ions generated in the sputter ion source and in the Van de Graaff accelerator tubes.

3) Studies of preparation of sputter source material for carbon ion and beryllium ion production.

4) Ion-optics studies of the low energy, or ion injection, portion of the source and tandem, primarily to find a solution to the important problem of normalization.

At the time of writing this report, we believe we have found adequate solutions to 1,2 , and 3 , but the last--the normalization problem--is still not satisfactorily solved. Apparatus is now under construction, and is soon to be tested, which will enable us to monitor continuously a "normalizing" beam (as, for example, ${ }^{12} \mathrm{C}^{-}$). Simultaneous measurements must be made of the normalizing beam in the low-energy section (1.e., prior to high-voltage acceleration) and of the desired radioisotope beam (as, for example, the ${ }^{14} \mathrm{C}$ ion counting rate) following acceleration and subsequent momentum selection or other ion discrimination. This avoids a serious problem: if both (say) ${ }^{14} \mathrm{C}$ and ${ }^{12} \mathrm{C}$ were simultaneously accelerated, the large ${ }^{12} \mathrm{C}$ beam would generate a tremendous background of ions which could masquerade as ${ }^{14} \mathrm{C}$ and which, in the absence of strenuous additional separation techniques, would mask or seriously distort the ${ }^{14} \mathrm{C}$ beam.

While this method of normalization avoids a serious ion background problem, it opens the possibility of erroneous ${ }^{14} \mathrm{C}$ measurements because the Van de Graaff accelerator transmission is not constant in time. To eliminate the effects of variable transmission, we intend to compare the unknown source with a standard source. The two sources must be alternated many times for each measurement in order to reduce the effects of variable transmission to a level commensurate with statistical counting errors. 
Equipment for a definitive test of this normalization scheme is under construction and will be tried soon.

Our source preparation studies have led to several new interesting results:

1) Large negative $C$ beams can be made only from "graphitized" source material. Amorphous $C$ gives $1 / 5$ to $1 / 10$ as much beam.

2) The negative $C$ beam magnitude is independent of the relative orientation of the graphite crystal planes. This latter conclusion was reached by using sources made of Grafoil 1 carbon material (a graphite foil material in which the crystals are aligned parallel to the foil surface).

3) Very pure $C$ source material can be successfully prepared and deposited on a on a sputter source disc by converting $\mathrm{CO}_{2}$ quantitatively into $\mathrm{CO}$.and cracking the $\mathrm{CO}$ in a glow discharge. While good negative $\mathrm{C}$ ion yields have been obtained with sources containing only 1 to $2 \mathrm{mg}$ of $\mathrm{C}$ deposited directly in this way, higher yields and greater durability are achieved by molding the carbon under high pressure, followed by graphitizing the deposited $C$.

4) Metallic Be sources giving $\mathrm{Be}$ ion yields ( $\mathrm{Be}^{-}$or $\mathrm{BeO}^{-}$) as large as those from commercial Be metal have been prepared from BeO. This is accomplished by heating the $\mathrm{BeO}$ with $\mathrm{Mg}$ powder to reduce the $\mathrm{BeO}$ (800-9000 C) and subsequently evaporating the Be onto a sputter source disc $\left(1300-1400^{\circ} \mathrm{C}\right.$ ). The required amount of $\mathrm{Be}$ is 10 to $20 \mathrm{mg}$ (before reduction).

We have successfully made comparisons of ${ }^{14} \mathrm{C} /{ }^{12} \mathrm{C}$ ratios for several carbon samples from woods of different origins and of $10_{\mathrm{Be}} /{ }^{9} \mathrm{Be}$ ratios for beryllium samples of various origins. The ${ }^{14} \mathrm{C}$ counting rates varied from about 40 counts/min/ $\mu \mathrm{A} \quad{ }^{12} \mathrm{C}^{-}$for 1964 wood to $<0.3$ counts/min/ $/{ }^{12} \mathrm{C}^{-}$for graphite. Although in our comparison the measured relative values of the ${ }^{14} \mathrm{C} / 12 \mathrm{C}$ ratios for the various samples agreed with the expected relative values within the statistical uncertainties, the fact that these uncertainties (counting statistics excluded) were in the range of $+5 \%$ to $\pm 15 \%$ indicates the need for a better normalization procedure as discusse $\bar{d}$ above. Somewhat more consistent results were obtained for $10_{\mathrm{Be}} /{ }^{9} \mathrm{Be}$ ratios, for which normalization was accomplished by cycling both the inflection system and the high-energy magnetic analysis system, so that the $10_{\mathrm{Be}}$ counting rates could be normalized to analyzed ${ }^{9} \mathrm{Be}^{+3}$ ion beams. Normalization studies of this method are continuing.

lihe results on carbon and beryllium, together with a description of our present radiometric dating system and further details in regard to source preparation and ion yields, were presented in two papers ${ }^{2}, 3$ given at the Tenth International Radiocarbon Conference held at Bern and Heidelberg, August 19-26, 1979. 
Work is continuing toward an automated ion source that will allow rapid alternation of samples without cross-contamination; an ion injection system with higher mass resolution to enable us to work with heavier radioisotopes such as $26 \mathrm{Al},{ }^{36} \mathrm{Cl}$, and $53 \mathrm{Mn}$; an ion velocity filter to reduce the background of unwanted ions in the radioisotope beams; and a high-resolution ion detector system for use with all elements heavier than carbon.

\section{References}

+ Department of Geological Sciences and Quaternary Isotope Laboratory, University of Washington.

1. Union Carbide Corporation, Carbon Products Division, Chicago, IL 60606.

2. G.W. Farwell, T.P. Schaad, F.H. Schmidt, M.-Y.B. Tsang, P.M. Grootes, and M. Stuiver, Radiocarbon, Vol, 22, No. 2 (1980) (in preos).

3. P.M. Grootes, M. Stuiver, G.W. Farwe11, T.P. Schaad, and F.H. Schmidt, Radiocarbon, Vol. 22, No. 2(1980) (in press).

\section{Van de Graaff Acceleratior Operations and Development}

\section{Staft}

The following major projects have been carried out during the past year to improve the Van de Graaff accelerator. All are described in other sections of this report as indicated.

1) reconstruction of the terminal voltage regulator (9.7),

2) improvements to the polarized ion source $(8.4)$,

3). improvements to the sputter sources ( 8.1 and 8.5 ),

4 ) installation of a gamma radiation monitor system (8.7), and

5) study of low energy optics (8.6).

In addition, we have made the following modifications to the accelerator:

1) improved the television readout of the injector terminal source parameters,

2) added a voltage regulator to stabilize low energy. optical element power supplies,

3) installed an improved high energy beam stop, the so-called "flap", and

4) rebuilt the vacuum control system on the 10 inch scattering chamber.

During the year from April 16, 1979 to April 15, 1980, the tandem was operated 5612 hours; and the injector was operated 60 hours. Other statistics of the accelerator operation are given in Table 8.2-1. 
Table 8.2-1: Tandem Accelerator Operations

April 16, 1979 to April 15, 1980.

\section{Activity}

A. Nuclear Physics Research

1) Light Ions

2) Polarized Ions

3) Heavy Ions

4) Radiochronology

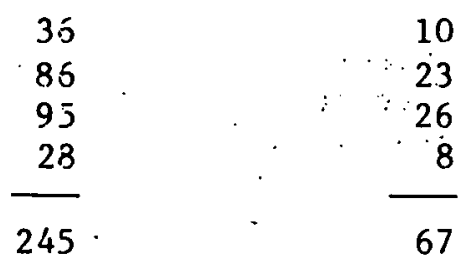

B. Outside Users

1) Battelle Northwest Laboratories

2). Hanford Engineering Development Laboratory (Westinghouse)

3) University of Washington Nuclear Engineering

4) University of Alberta

Total

$\begin{array}{lll}4 & 1 \\ 2 & \therefore<1 \\ 1 & \quad \frac{<1}{7}\end{array}$

c. Other

1) Accelerator Development

\begin{tabular}{rr}
8 & 2 \\
35 & 10 \\
51 & 14 \\
\hline 95 & 26 \\
\hline \hline 356 & 100
\end{tabular}

2) Accelerator Maintenance

3) Unscheduled Time

$$
\begin{aligned}
& \text { Total } \\
& \text { GRAND TOTAL }
\end{aligned}
$$




\section{Cyclotron Operations and Development}

H. Fauska, C. Linder, G. Rohrbaugh, G. Saling, R. Stowell,. P. Wiest, and W.G. Weitkamp

The 60 in. cyclotron, now after 30 years of continuous use the oldest operating conventional cyclotron in the world, continues to provide beams for cancer therapy and other applications described in Sec. 7 of this report.

Fearful that the 30 year old motor-generator set providing the main magnet current would fail catastrophically during the year, we replaced it with a solid state power supply. Transformers and rectifiers for this supply were purchased; error sensing amplifiers, the series pass transistor bank and transistor drivers were built in-house. A rough regulator to drive the primary power variac was also constructed and installed. The new system has operated satisfactorily for more than nine months.

In addition, we have:

1) replaced antique power supplies on the up-down and focus magnets,

2) replaced the duct diffusion pump,

3) replaced the heaters in the main diffusion pump,

4) overhauled the oscillator frequency control circuit, and

5) solved sparking problems around the main oscillator tube.

At present, we are working on an MMR based regulator for the main magnet, and installing a TI 550 controller to replace the old banks of relays controlling the vacuum and safety interlocks. Work on both these projects has been slowed by the current high demand for cyclotron beam time.

The cyclotron operated 1019 hours between April 16, 1979 and April 15, 1980. Other statistics of cyclotron operations are given in Table 8:3.1. 
Table 8.3-1: Statistics of Cyclotron Operations April 16, 1979 to April 15, 1980

\section{Activity}

Division of Medical Radiation Physics (Cancer. Therapy)

Division of Nuclear Medicine (Total Body Calcium)

Division of Nuclear Medicine (Isotope Production)

Department of Environmental Health

Oregon State University

- University of Illinois

Scheduled Maintenance

Inscheduled Maintenance

TOTALS
Days

135

15

10

5

1

3

10

20

199
5

10

Percent

68

.

8

5

2.

$<1$

2

100 
4. Polarized Ion Source Developments

W.B. Ingalls and T.A. Trainor

The failure of the high voltage insulation beneath the duoplasmatron region resulted in a two week shutdown of the ion source this year. The original insulation consisted of a $3.2 \mathrm{~mm}$ thick sheet of PVC laminated to a lower PVC sheet $9.5 \mathrm{~mm}$ thick. Coolanol trapped in the interface during the first years of operation contributed to the establishment of a carbon track through the thin sheet, along the interface to the edge, and down the thick sheet to the supporting metal undercarriage. The ion source and old insulation were supported with the overhead crane; the undercarriage was lowered to accomodate the insertion of $12.7 \mathrm{~mm}$ thick strips of PVC between the old insulation and the undercarriage to accomplish an expedient repair. The removable flooring around the ion source was modified to accommodate the new insulation, which was purposely made approximately $3.8 \mathrm{~cm}$ wider than the old to provide a longer breakdown path length.

Intermittent leaks in the forevacuum of the ion source have been eliminated by the installation of a new forevacuum manifold. The new manifold is mounted securely to the ion source frame and connected to the three diffusion pumps via $3.8 \mathrm{~cm}$ ID reinforced PVC hose.1 A spiraled $75 \mathrm{~cm}$ long piece of the same hose joins the manifold to the forepumps. The new hose is much more flexible "than corresponding ID thick-walled rubber tubing and the vibrational coupling of the forepump with the remainder of the ion source has been significantly reduced.

A low-volume stainless steel source gas manifold of hard solder and compression fitting construction was installed to eliminate recurring leak and/or contamination effects observed in the duoplasmatron operation. The manifold is connected to the gas inlet of the duoplasmatron via a stainless steel bellows and a $5 \mathrm{~cm}$ piece of thick-walled pyrex tubing.

In conjunction with the new forevacuum and source gas manifolds a simplified vacuum interlock controller was installed. The front panel also incorporates a Varian model 810 thermocouple controller for forevacuum monitoring and interlock protection and a 0 to $20 \mathrm{~mm} \mathrm{Hg}$ Hastings-Raydist ${ }^{2}$ thermocouple gauge for duoplasmatron source gas monitoring.

A major source of difficulty with the wicking cesium canal ${ }^{3}$ has been leakage of cesium into the duoplasmatron vacuum box. The molten cesium evidently travels by capillary action along the threads of the valve stem used to isolate the oven from the canal and then drips to the optical bench below the oven.

The oven-valve assembly was replaced by a new one which incorporates the stainless steel bellows assembly from a Hoke model 4251 NGY valve to provide a seal between the valve stem and the surrounding vacuum system.

We have continued to upgrade the electronics portion of the ion source with well-regulated commercial power supplies. This year we have added a 40 volt, 5 amp supply $\mathrm{y}^{4}$ to replace the duoplasmatron magnetic field supply and a $40 \mathrm{kV} \mathrm{RF}$ regulated supply $y^{5}$ for the first gap of the acceleration tube. 
$\underline{\text { References }}$

1. Trade name "Kanaflex."

2. Mode1 DV-4.

3. Nuclear Physics Laboratory Annual Report, University of Washington (1979), p. 137.

4. Hewlett-Packard, Model 6266B.

5. Spe1lman High-Voltage Electronics Corp., Model RHR 40P30.

5. Sputter Ion Source Development

J.F. Amsbaugh, D.W. Storm, and W.G. Weitkamp

Our intention at the beginning of this year was to complete the work on the developnent sputter sourcel and install it on the tandem. We have been delayed in this plan, because we found that optimizing the source geometry by trial and error was not productive. Consequent1y, we spent a considerable effort in computing the trajectories for both the cesium ions and the negative ions from the source. Along with these computations, we have carried out a series of measurements to verify the calculations and to determine optimum geometries for the cesium beam. Now that we have made this progress in obtaining a deeper understanding of the source optics, we expect to be successful in finishing the development work in the near future. Already, we have more than doubled the beam intensity produced by the development source.

Along with the work spent studying the details of the source optics, it was also necessary to make various electro-mechanical improvements in the development source to obtain satisfactory reliability of operation. A number of high voltage feed throughs were replaced with sturdier versions, and a number of electrode supports were modified to shield their insulators from the cesium. Finally, the cesium steering power supplies were modified so they could both supply and sink current. Now the source operates reliably over the entire range of the focusing and accelerating voltages.

A photograph of the ion source electrodes is shown in Fig 8.5-1. Our first calculational effort, when we found that carbon beams were 1imited to 5-6 $\mu \mathrm{A}$ for a number of trial configurations, dealt with the optics for the negative ion beams. The calculations were carried out using the program CYSYM. ${ }^{2}$ This program was modified extensively to include the reflection of the cesium beam and to deal with the small scale structure in the vicinity of the cesium ionizer and the negative ion source pellet. The program solves Laplace's equation for the axially symmctric clectrode geometry, and then carries out a numerical integration to determine particle trajectories. Another modification, done to make efficient use of the new VAX computer, involved storing the results of the electric potential calculation in a disc file, and then using these for a number of trajectory computations.

From these calculations we determined that for the various electrode configurations we had tried, the system should be operating with a narrow 


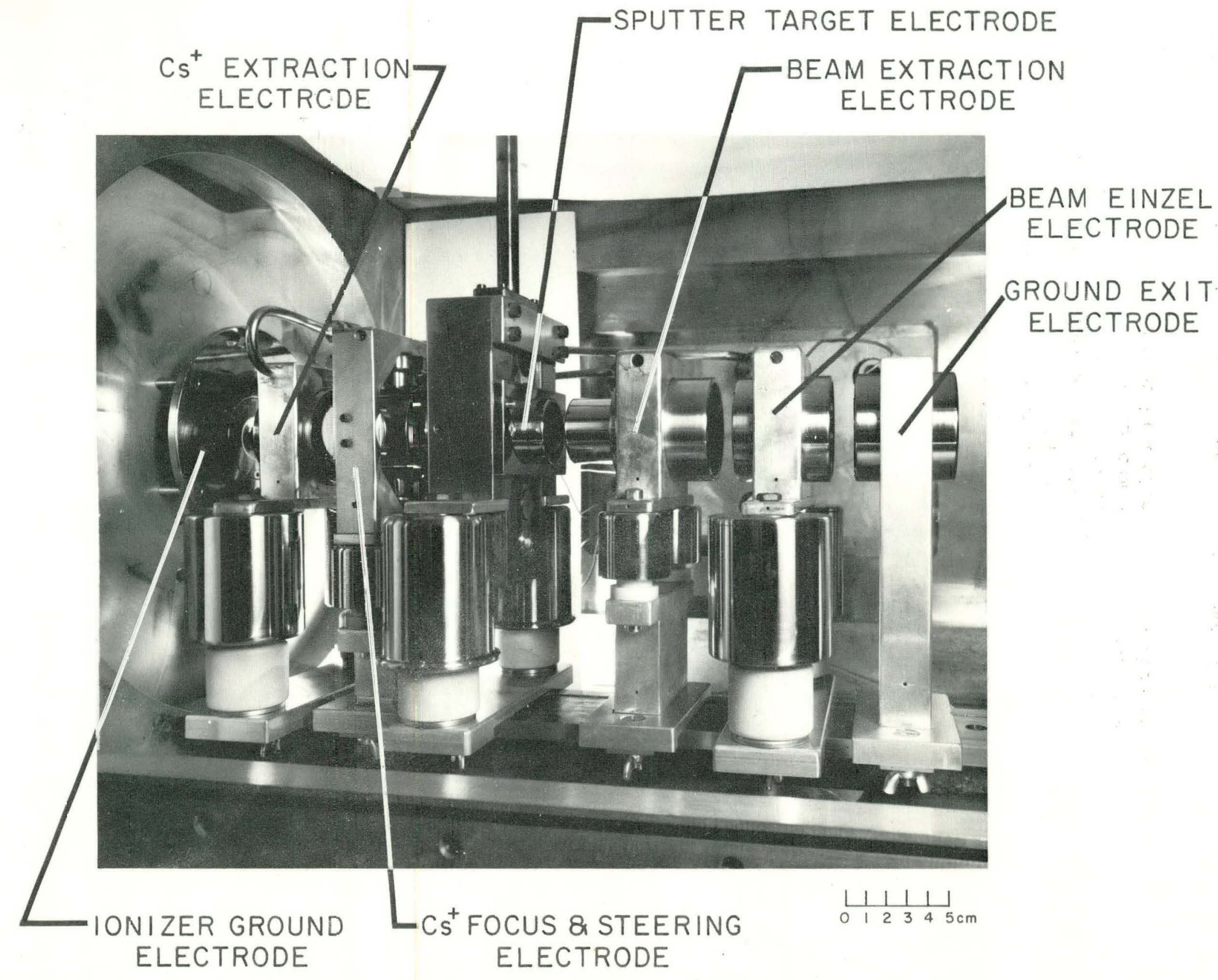


negative ion beam with negligible aberrations, and there should be no problem with any of the beam being intercepted in the ion source. Furthermore, we carried out a set of computations for the UNIS sputter source used on the tandem to determine the emittance figure of the beam from that source. The result was that the particles occupy the phase space illustrated in Fig. 8.5-2. A number of measurements on the low energy beam of the tandem gave results consistent with this prediction.

Since the optics for the negative ion beam seemed to be satisfactory, we investigated the situation of the cesium beam. Most of our tests used an off axis channel to transmit the cesium past the sputter target; the negative ion accelerating electrode was biased to reflect the cesium back onto the sputter target. Other tests with six holes symetrically placed about the pellet, as in the source on the tandem, gave similar beam intensities. The intention with the single channel geometry was to use the cesium transport system, which includes a lens with steering capability, to focus and steer the cesium beam through the channel. Then, using the variable reflection voltage, one would focus this intense cesium beam onto a small spot, since the reflection potential should act as a concave mirror.

Since it is difficult to measure the intensity of the cesium beam striking the sputter target, because there are much higher currents of electrons due to secondary emission and field emission, we approached the cesium problem from three directions. First, we measured the cesium beam passing through the channel near the sputter target. This was done by putting a Faraday cup with appropriate biased electrodes down stream of the channe1, as indicated in Fig. 8.5-3. We determined that, at a Cs oven temperature of $290^{\circ}$, we could produce $0.4 \mathrm{~mA}$ of cesium beam which could be focused through a $1.5 \mathrm{~mm}$ aperture at the exit of the channel. Normally the source operates with a range of oven temperatures between $275^{\circ} \mathrm{C}$ and $340^{\circ} \mathrm{C}$.

Second, we calculated the trajectories of the reflected cesium ions and determined what values of $r$ and $\theta$ Cs ions would have to have at the plane of the cesium channel exit in order to hit a $2.4 \mathrm{~mm}$ diameter pellet on the axis. The results of these calculations indicate that the reflection lens has very large aberrations, but that the cesium ions in a particular $35 \mathrm{~mm}-\mathrm{mr}$ area of the phase space will hit the target. Similar calculations for the UNIS reflection geometry indicate that the holes through which the cesium passes were located near the optimum position for that system.

Third, we carried out a series of calculations on the cesium transport system to see how we could match the cesium beam. to the phase space that would be reflected back onto the sputter target. The conclusion of these calculations was that the matching required changes in the geometry of the cesium ionizer. By moving the grounded electrode near the ionizer tip we can make large changes in the strength of the lens associated with the cesium acceleration gap. Consequently, we have made a mechanism that permits us to move that electrode when the source is running. According to the calculations, the adjustment of this electrode, along with the focusing and steering of the cesium einzel lens, will enable us to produce a cesium beam that will pass through the channel and be reflected to a small spot. As a test of this scheme, we have measured the emittance of the cesium beam by focusing it through a pair of holes at the channel position. These apertures were $17.8 \mathrm{~mm}$ apart, and we found that with 
EMITTANCE AT EXIT OF UNIS SPUT, ION ENERGY $10 \mathrm{eV}$

ION ENERGY $=25.01 \mathrm{heV}$

EINZEL LENSE VOLTAGE $=-16.0 \mathrm{kV}$.

NORMALIZEO EMITTANCE $=4.438 \mathrm{~mm}-\mathrm{mrod}-\mathrm{MeV}^{\mathrm{t}}$

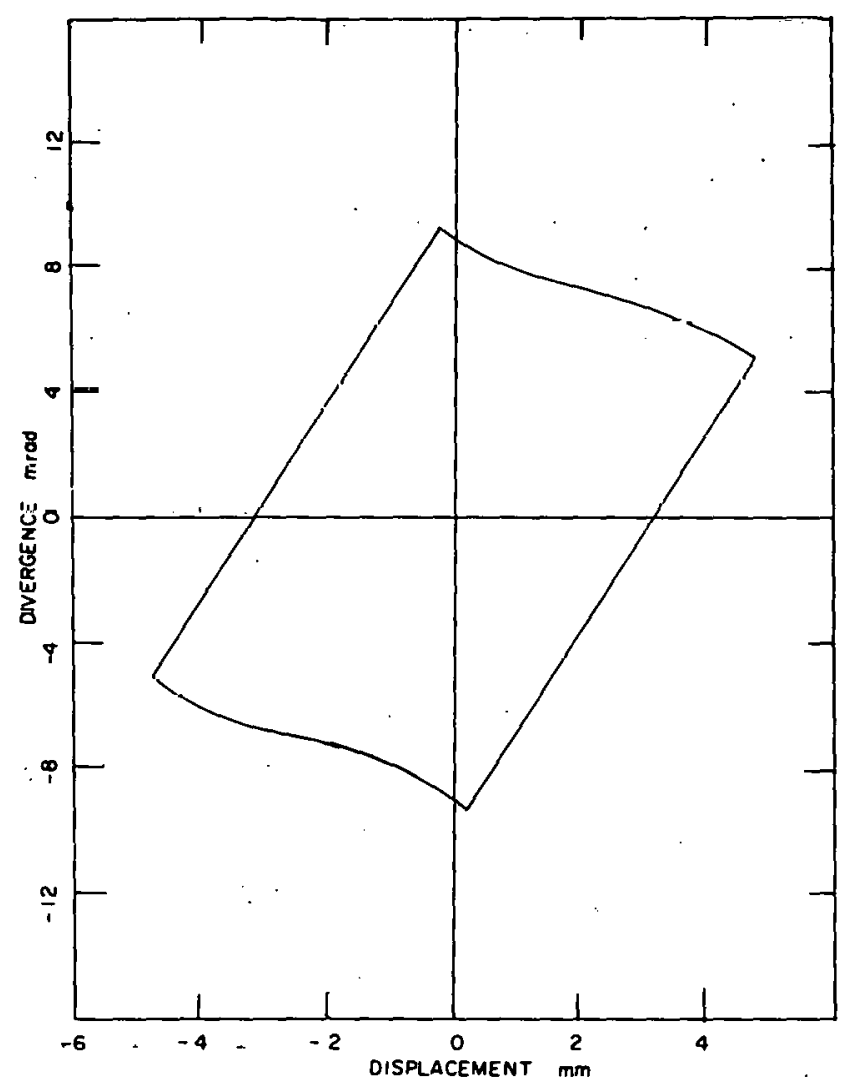

Fig. 8.5-2: Emittance figure for the UNIS ion source. used on the tandem.

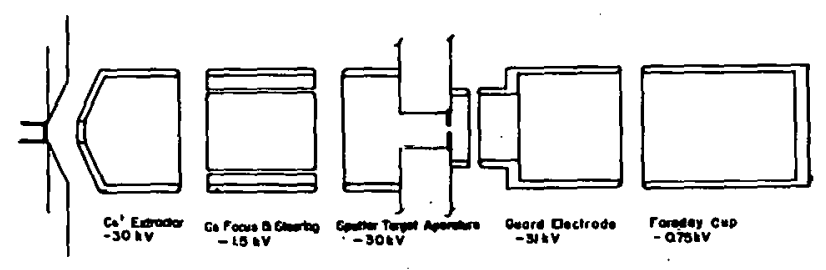

Fig. 8.5-3: Modification to the development source permitting measurement of the cesium beam transmitted through the apertures near the sputter pellet. 
appropriate settings of the ground electrode position and the focusing voltage, the entire cesium beam $(0.4 \mathrm{~mA})$ would go through a pair of $1.5 \mathrm{~mm}$ holes. This corresponds to an emittance of $126 \mathrm{~mm}-\mathrm{mr}$.

At present we have resumed tests in which the cesium is reflected back onto the sputter pellet. We plan to determine the optimum cesium beam optics to produce the maximum negative ion beam and will measure the emittance of that beam. Various configurations will be studied, including the single cesium channel and the six holes for the cesium, as well as a conventional cone geometry, in which there is no cesium reflection. Although the tests have just begun, we have already obtained a $20 \mu \mathrm{A}$ beam with good emittance from the single cesium channel geometry.

\section{References}

1. Nuclear Physics Laboratory Annual Report, University of Washington (1979), p. 138 .

2. Nuclear Physics Laboratory Annual Report, University of Washington (1972), p. 40 .

\section{Low Energy Optics Studies}

J.F. Amsbaugh, F.H. Schmidt, D.W. Storm, and W.G. Weitkamp

The present configuration for the low energy optical system for the tandem is illustrated in Fig. 8.6-1. The optical elements include the inflection magnet, which performs a weak focusing, the five inch einzel lens, and the two inch bore quadrupole triplet. The locations of these elements have been determined by various physical constraints. They are intended to function as a telescopic system, to produce a waist of appropriate size at the location appropriate to the terminal voltage. This system can prepare beams from the direct extraction ion source to yield nearly $100 \%$ transmission through the terminal. For the polarized ion source, the transmission can be as high as $90 \%$ at some terminal voltages, but it is poor at low voltage. The transmission for the sputter ion source can be over $30 \%$ at terminal voltages of $7 \mathrm{MV}$, but is less at other voltages. We have undertaken a series of measurements and calculations to determine the emittance of the sputter ion source and to determine how to improve the transmission of the sputter source. As a result of these studies, we are presently planning to replace the quadrupole doublet with a triplet, and to add a gridded lens to the end of the low energy beam tube.

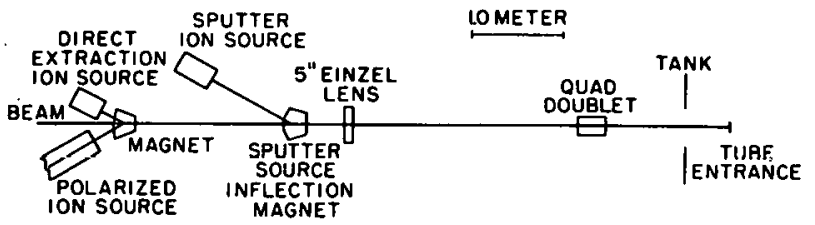

Fig. 8.6-1: Layout of the present low energy beam optical system. 
Calculations of the emittance of the sputter ion source were carried out, and are presented in Section 8.5 of this annual report. The calculations indicate that the emittance of the source is determined by the size of the source spot for the lons and by the typical sputter energy of $10 \mathrm{eV}$. Aberrations in the source optics are not important, and nearly all the ion beam will pass through the magnet apertures. The calculations predict that the maximum transmission through the magnet will occur at the same values for the source parameters for which the maximum is observed. In addition, the spot size measured at a point $35 \mathrm{~cm}$ from the source exit is consistent with the predicted value.

The acceptance of the accelerator is determined by the stripper aperture and by the entrance of the low energy beam tube, in conjunction with the immersion lens at the beginning of the acceleration tube. At all but the lowest terminal voltages, the entrance lens of the beam tube is strong, and the area of the acceptance figure is substantially larger than the emittance of the ion sources. For example, at $25 \mathrm{keV}$ ion energy, the sputter source produces a 12 $\mathrm{mr}-\mathrm{cm}$ emittance area, while the acceptance of the accelerator is greater than that figure for terminal voltages above $0.7 \mathrm{MV}$ (and for the same $25 \mathrm{keV}$ ions). 1 The size and location of the entrance pupil has been calculated previously as a function of terminal voltage and ion energy, and is indicated in Fig. 8.6-2. The same calculations were used to determine the acceptance. These calculations predict that the entrance pupil will be located at the slits on the beam line just at the tank entrance for $25 \mathrm{keV}$ ions and with $2.0 \mathrm{MV}$ on the terminal. As a check, this result was confirmed experimentally by measuring the opening at which beam began to hit the slits, as a function of terminal voltage, when the. low energy optical system was focused for maximum transmission.

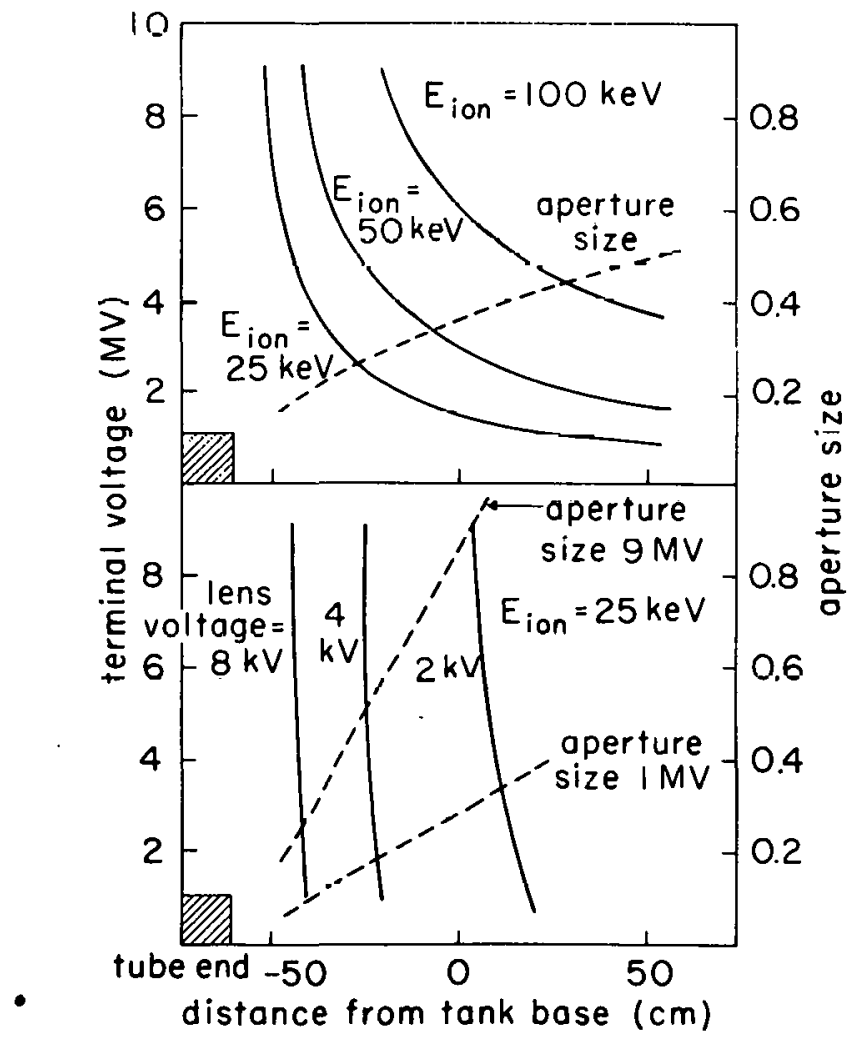

Fig. 8.6-2: In the top part the position of the entrance pupil without the gridded lens is plotted vs. terminal voltage with solid lines for thrce ion energies. Also, the size of the pupil is plotted vs. position with the dashed line. In the bottom part, the position vs. terminal voltage is indicated for $25 \mathrm{keV}$ ions with the gridded lens for a number of grid biases. The pupil size is shown with dashed lines for two limiting terminal voltages. 
Since nearly all the focusing results from the field gradient at the end of the beam tube, and since this lens strength depends on the ratio of terminal voltage to ion energy, it is a quite general result that the entrance pupil will: first, be much smaller than the stripper aperture and fairly near the tube entrance at high terminal voltage, and second, move away from the tube (toward the ion source) and get larger as the terminal voltage is lowered (or the initial ion energy is increased). Thus the task of the present low energy optical system is to produce a waist at the appropriate point, dependent on terminal voltage.

For high terminal voltage this waist is small and far from the quadrupole doublet. Under these conditions the bore of the doublet limits the convergence angles associated with the waist, so it is impossible to put the entire beam through the quadrupole and into the entrance pupil. At lower terminal voltages, the waist is larger and nearer the quadrupole lens. Thus one might expect that at some low terminal voltage, one could match the ion beam emittance to the acceptance of the accelerator. However, since the principal planes of the quadrupole doublet with short focal length are shifted outside the lens on the converging side (see Fig. 8.6-3), the result is that in one plane the waist is small, but the converging angles are too big, while in the other plane the waist is still too big.

In order to rectify this problem, we are building a quadrupole triplet lens to replace the doublet. In addition, we are preparing to install a gridded lens at the beam tube entrance. Using the gridded lens, we will be able to maintain the entrance pupil at a convenient location for all terminal voltages so that the triplet (with a three inch bore) will be able to focus all the beam from any of the ion sources into the accelerator acceptance, for terminal voltages above about 1 MV for the sputter ion source and above about 2 MV for the polarized source. An alternate approach would be to float the source at a variable potential. For maximum terminal voltage ( 9 MV) a sputter source voltage of 25 $\mathrm{kV}$ (yielding ions of $50 \mathrm{keV}$, since the source $\mathrm{r}$ uns with $25 \mathrm{kV}$ accelerating voltage) would be required to match the source emittance figure to the accelerator acceptance figure, assuming the quadrupole triplet were to be filled to the 3 inch diameter. At this voltage the source emittance would be reduced to $8.5 \mathrm{~cm} \mathrm{mr}$, and the beam convergence angle, determined by the triplet bore and entrance pupil location would be $35 \mathrm{mr}$. The radius of the entrance pupil would be $1 \mathrm{~mm}$. For lower terminal voltages the source voltage would be lowered, to prevent the entrance pupil from getting too close to the quadrupole triplet. We have decided to pursue the use of the gridded lens in order to avoid the technical difficulty of floating the entire sputter source at high voltage. Consequently we have calculated the position of the entrance pupil as a function of terminal voltage and grid voltage. The results are shown in the lower part of Fig. 8.6-2.

It is clear that the position of the pupil is nearly independent of terminal voltage with a gridded lens. It is also clear that the pupil can be located at any desirable location by applying a modest voltage to the grid. For example, with a bias of about $10 \mathrm{kV}$, the entrance pupil will be near the tank entrance, and about $3 \mathrm{~mm}$ in diameter. The maximum convergence angle permitted by the quadrupole triplet will be $35 \mathrm{mr}$, while the $12 \mathrm{~cm}-\mathrm{mr}$ emittance of the sputter source will be focused into a $4 \mathrm{~mm}$ spot with convergence angles around $20 \mathrm{mr}$. 


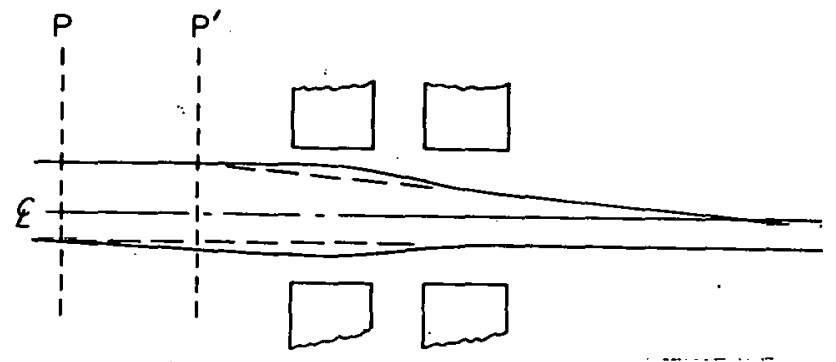

F1g. 8.6-3: The principal planes of a quadrupole doublet are located outside of the converging side when the lens is focusing to a nearby point. Thus the focal length in the convergingdiverging plane is much longer than that in the divergingconverging plane, and so the magnifications and convergence angles are quite different in the two planes.

Installation of a gridded lens does not increase the acceptance of a beam tube; on the contrary some beam is lost to the wires of the grid. But it is expected to enable us to match the source emittance figure to thc accelerator acceptance quite well over the full range of terminal voltage.

\section{Referénces}

1. Nuclear Physics Laboratory Annual Report, University of Washington (1977).

\section{Gamma Radiation Monitor System}

\section{S.K. Lamoreaux and W.G. Weitkamp}

In the past the tandem radiation system protection has been based on neulivil wonfiors. Ihese monitors give three readouts: (1) a meter reading directly in $\mathrm{mr} / \mathrm{hr}$ on a logarithmic scale, (2) a system of interlocks which turn off the beam if shielding doors are opened improperly, and (3) an audible clicking sound--the frequency of the clicks in $\mathrm{Hz}$ is equal to the radiation dose rate in $\mathrm{mr} / \mathrm{hr}$. For many accelerated beams, neutron flux is a reliable indicator of total radiation hazard. However, heavy ion beams can produce high gamma ray fluxes without producing significant neutron fluxes. Consequently we have designed and installed a gamma ray monitoring system to complement the neutron systen.

The gamma radiation intensity is measured with ion chambers salvaged from old "Culle Ple" portable detectors, with all voltages provided by regulated power supplies instead of batteries. The chamber current is measured by a MOS op-amp electrometer circuit. The output is fed into a 741 op-amp with a germanium diode in the feedback loop to provide a logarithmic response. The output voltage is displayed in the accelerator control room on a 0-1 ma meter with a logarithmic scale. 
The meter signal is compared to two different voltages corresponding to 10 $\mathrm{mr} / \mathrm{hr}$ and $100 \mathrm{mr} / \mathrm{hr}$. The comparators consist of $741 \mathrm{op}$ amps with the signal fed to one input and a settable voltage at the other. Using the $741^{\prime} \mathrm{s}$ in this manner gives a sharp transition at the threshold point. The outputs of the comparators are fed into a logic circuit which lights different colored LED's corresponding to different radiation levels, and triggers a relay when the radiation goes above the preset value $(100 \mathrm{mr} / \mathrm{hr})$. The relay activates the accelerator door interlocks.

An optional audio alarm circuit has also been designed. The meter signal is fed into a voltage-to-frequency converter. The converter consists of an integrator, a comparator, and a monostable multivibrator. The voltage is fed into the integrator. After a time interval proportional to the input voltage, the output voltage of the integrator will go above the threshold voltage of the comparator. The comparator then triggers the monostable multivibrator which generates a square pulse of precise width and height. The pulse discharges the integrating capacitor. Thus, the circuit forms an oscillator with frequency proportional to the input voltage. The multivibrator pulses provide the output of the converter. The pulses generated are fed into a pulse shaper, amplifier and loudspeaker identical to those used in the neutron monitors.

The drift of the electrometer circuit is less than $0.5 \mathrm{mr} / \mathrm{hr} / \mathrm{month}$, and seems to be tolerable. The entire system has given satisfactory performance for a substantial fraction of the year, and is now being expanded to provide monitoring in the experimental areas of the Laboratory.

\section{References}

1. Nuclear Physics Laboratory Annual Report, University of Washington (1965), p. 63 .

\section{UNIS Ion Source Improvements}

J.F. Amsbaugh, F.H. Schmidt, D.W. Storm, and W.G. Weitkamp

A number of changes were made with the Extrion UNIS (Universal Negative Ion Source) on the tandem. Previously the source had a high failure rate, with a record 210 hours between maintenance. As a result of the improvements, it has run over 600 hours, at which time the cesium supply was exhausted. More cesium was added, but the entire source was not dismantled and cleaned. It has subsequently run another 225 hours, and has not failed yet. The main failure mode previously resulted when an insulating standoff supporting the decel electrode (see Fig. 8.8-1) short circuited, because sputtered metal was deposited on it. When this electrode was shorted to the extraction voltage, negative lons would strike the cesium ionizer, sputtering a hole in it. Then the source would be flooded with cesium. To correct this problem, a shield was built to protect the insulator from direct bombardment by sputtered material. 


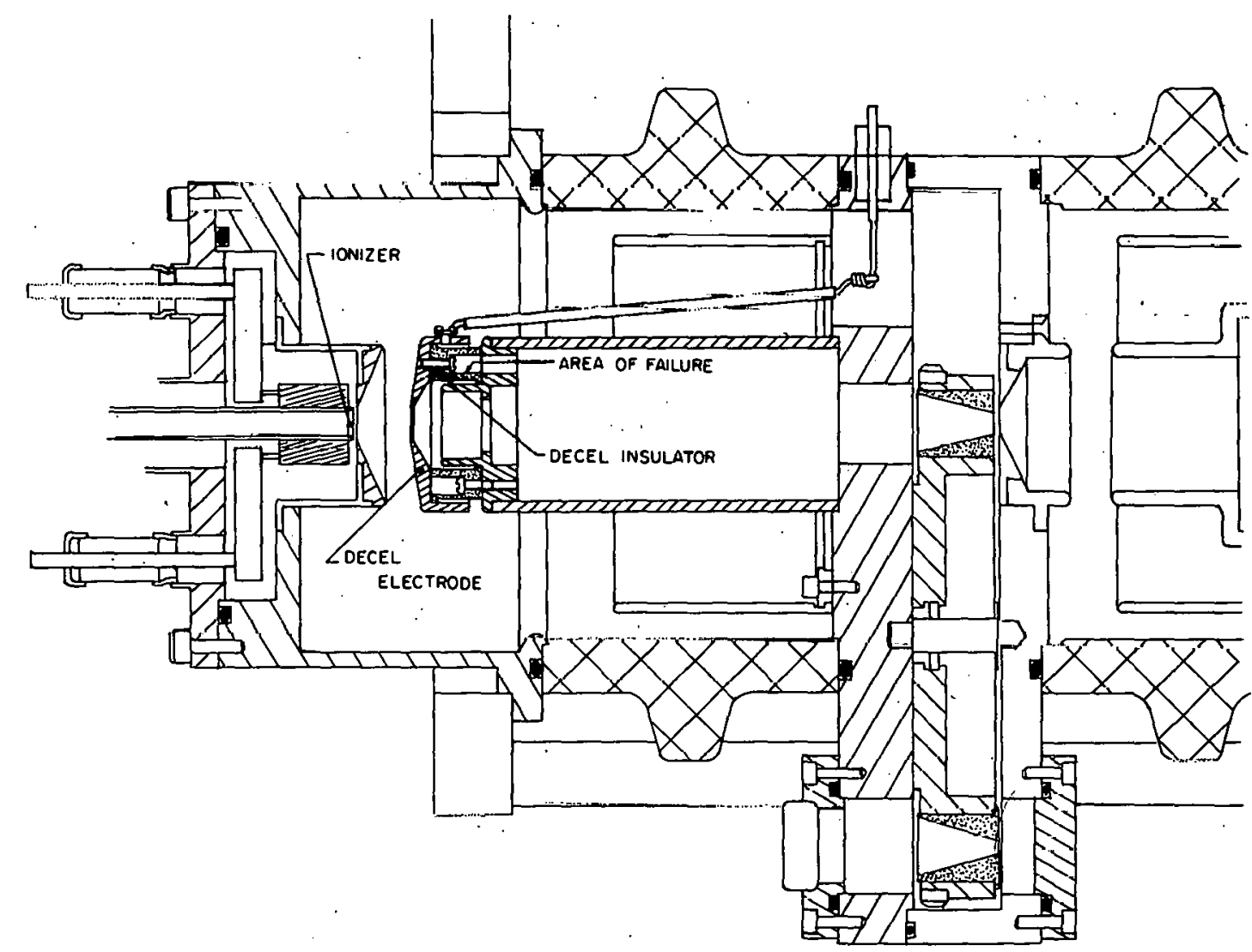

Fig. 8.8-1: Schematic diagram of the UNIS source showing the electrodes. 
Another reason for the long time between failures is probably because of the extensive use of the six hole reflection geometry, rather than the cone geometry. In the latter, the cesium beam strikes the upstream side of the material to be sputtered, and the negative ion beam is extracted through a hole through the material. In addition, there is substantial material sputtered into the part of the source upstream of the sputter cone. With the six hole reflection geometry, on the other hand, the upstream side of the source holder is tantalum, which has a low sputter yield. The cesium passing through the six holes is then reflected onto the sputter pellet. Most of the negative ions produced are accelerated. Neutral sputtered material can coat various electrodes, but the insulators are shielded along the line of sight from the sputter pellet.

A final improvement relating to the reliability of the operation of the source was the installation of a spark suppressor on the reflection electrode. Originally this electrode was at ground potential, and the $-25 \mathrm{KV}$ potential difference between it and the sputter target produced the acceleration of the negative ions. With the reflection geometry, the reflection electrode is biased at a few hundred volts positive, to reflect the cesium ions, which originate at ground potential, back onto the $-25 \mathrm{KV}$ sputter target. The spark arrestor prevents damage to the reflection supply in the event of a discharge between the reflection electrode and the sputter target electrode.

In order to improve the ease of operation and stability of the UNIS we built a feedback controlled cesium oven. The control is an Omega temperature controller, which uses a thermocouple for a sensing element, and produces a proportional error signal, which is used to drive an SCR controller which supplies the heater current. The thermocouple well is positioned between the heater and the oven, to insure stability of the system and the temperature regulation is present $1 \mathrm{y} \pm 2{ }^{\circ} \mathrm{C}$.

Finally, the freon cooling pump was replaced with a new, sturdier version, after the original pump failed. 


\section{INSTRUMENTATION AND EXPERIMENTAL TECHNIQUES}

\section{Development of a Large Area Position Sensitive Proportional Telescope}

\section{A. Lazzarini and V. Metag}

Our interests in coincidence heavy ion experiments has prompted us to produce several gas $\Lambda \mathrm{E}$-solid state $\mathrm{E}$ position sensitive proportional telescopes. Originally based on the design reported by Markham, ${ }^{1}$ we have modified this design to allow for a much larger active area. The solid state detectors we use are $900 \mathrm{~mm}^{2}, 100 \mu \mathrm{m}$ and provide us with an active area of $12 \mathrm{~mm} \times 26 \mathrm{~mm}$ for the telescopes. The charge resolution is adequate to resolve lighter elements in the $C$ and $O$ range. The limiting factor in the charge resolution arises from the large active arca; however for the experiments in which these counters have been used large solid angle was a more important consideration. In an experiment employing $1.4 \mathrm{GeV} 208 \mathrm{pb}+238 \mathrm{U}$, for example, the devices provided not only kinematic information $(\mathrm{E}, \theta, \phi)$, but also could distinguish light and heavy fission fragments from inelastically scattered target and beam particles. The photograph inFig. 9.1 shows the counters disassembled.

\section{References}

1. R.J. Markham, S.M. Austin, and H. Laumer, Nucl. Inst. and Meth. 129, 141 (1975).

Fig. 9.1-1: Photograph of disassembled counters. Metal scale is $15 \mathrm{~cm}$ long.

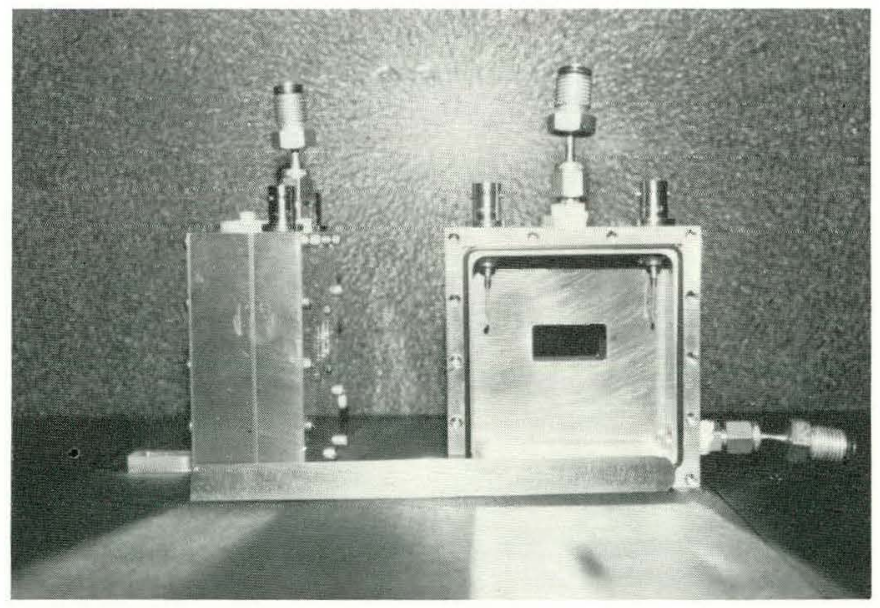




\section{Development of Cracked Ethylene Stripper Foils for the Tandem}

T. Bertram, G. Hinn, and W. Lynch

Radiation shrinkage is a major cause of stripper foil failure and, with respect to this problem, cracked ethylene foils have been reported to last as much as 30 times longer than the conventional vapor deposited foils. 1 Since it is possible to produce these foils in the laboratory, the project of developing the procedures and equipment for doing so was undertaken.

The foils are produced in a glow discharge, which is a stable gaseous electrical discharge in a gas at low pressure. The electrodes which maintain the reaction have a parallel plate configuration and are contained, along with the gas, in a vacuum chamber. For a particular gas, the relevant glow discharge parameters are: (1) the geometry and separation of the electrodes, (2) the potential difference between the electrodes, and (3) the pressure of the gas. It is believed that electrons emitted from the cathode surface ionize the gas, and the plasma set up by the ion pairs leads to plasma (atomic) polymerization. The observed film deposit on the cathode is thought to be the collective accumulation of the polymer. ${ }^{2}$ Foils may thus be obtained by retrieving this film, the thickness of which is determined by the amount of material deposited. Thus the foil thickness will depend on the reaction time as well as the other parameters mentioned.

The gas used for producing cracked ethylene folls is a mixture of $90 \%$ ethylene and $1.0 \%$ argon, the polymerization in this case being the cracking of the ethylene. The electrodes are parallel discs 6 inches in diameter; both aluminum and stainless steel electrodes have been used. Before the reaction, the cathode has a thin layer. $\left(20 \mu \mathrm{g} / \mathrm{cm}^{2}\right)$ of sodium chloride evaporated onto it to serve as a release agent. (The film is retrieved by floating.) The glow discharge parameters have been restricted to the following values:

1) electrode separation: 2.5"

2) anode potential: ground

cathode potential: $-2.3 \geq-3.0 \mathrm{kV}$

3) gas pressure: $55 \mu \mathrm{Hg}-6 \overline{5} \mu \mathrm{Hg}$

These values were chosen since they are comparable to those used at Chalk River.3 (Cracked ethylene foils have been produced at Chalk River and are currently in use there as accelerator stripper foils.) The remaining parameter of interest is the reaction time; the main emphasis of the project thus rests on determining the variation of foil thickness with reaction time. Once this relation is known, foils of desired thickness may. be produced in a straightforward and reproducible manner. 
The stripper foils used at NPL have areal densities of $10 \mu \mathrm{g} / \mathrm{cm}^{2}$ or less . With our present apparatus we have produced foils with densities between 7 $\mu \mathrm{g} / \mathrm{cm}^{2}$ and $27 \mu \mathrm{g} / \mathrm{cm}^{2}$. (Densities were determined by proton elastic scattering in which these foils, together with foils of known densities, were used as targets.) The reaction times for these deposits varied between 90 and 120 seconds. These times, however, cannot be correlated with foll thicknesses due to an instability in the reaction (discussed below). Some of those foils whose densities measured $10 \mu \mathrm{g} / \mathrm{cm}^{2}$ or less have been placed into the foil wheel in the accelerator terminal, and the in-beam lifetimes of the foils are currently being measured.

The reaction instability mentioned above is the tendency of the glow discharge to develop into an arc discharge. The arc is quite destructive, as it perforates the film deposit and leaves a pitted cathode surface. It also interrupts the steady accumulation of material to such an extent that the time dependence of the deposit thickness can not be measured. The arcing may be due to several factors:

1) electrode surface area exposed to gas is too large (i.e., reaction not confined to region between electrodes.)

2) flaws in the electrode surfaces producing high voltage gradients

3) chemical reaction involving the release agent.

Thus a new glow discharge apparatus has been designed and is under construction. It features insulated electrodes, only the inner faces of which are exposed to the gas. In addition, these inner faces are chrome plated. This new design addresses factors (1) and (2) directly; it addresses (3) to the extent that a smoother substrate requires less release agent. It is hoped that these modifications will elfminate the arcing problem and hence allow a determination of the foil thickness/reaction time relationship.

\section{References}

1. J.L. Gallant et al., Proceedings of World Conf. Int. Nucl. Target Devel. Society, $\overline{1979}$, to be published.

2. H. Yasuda, "Glow Discharge Polymerization," Thị Film Processes, J.L. Vossen and W. Kern (Eds.), Academic Press, 1978.

3. J.L. Gallant, private communication.

\section{Improvements to the Rabbit Target Transport System}

C.D. Hoyle

The rabbit is a pneumatic system for shutting a target between a bombarding site in the $0^{\circ}$ beam line and a counting site in cave 2 . The rabbit was previously used on the 00 beam line, but had been dismantled several years ago. The rabbit was improved and reinstalled on the 00 beam line this year. 
The rabbit consists of a section of $4^{\prime \prime}$ beam tube with a $1 / 2^{\prime \prime}$ square aluminum rabbit tube inserted through the beam tube perpendicular to the beam tube axis. The rabbit tube has entrance and exit holes so that the beam can travel through the rabbit tube, hit the target and exit the rabbit tube into a Faraday cup. The entrance and exit holes are covered with $1 / 4$ mil Havar foil since the beam is in vacuum and the target is not. The targets, mounted on balsa wood, carry that shuttle back and forth through the rabbit tube by applying compressed air. or suction to the rabbit tube.

Improvements to the rabbit include a new carbon aperture and a new system for applying the compressed air or suction to the rabbit tube. The new carbon aperture is a disc of carbon $1 / 8$ " thick, 2-1/2" in diameter with a $1 / 4^{\prime \prime}$ hole in the center for the beam. Before this aperture was installed it was possible to hit the Faraday cup without the beam passing through the target. It is now impossible to hit the Faraday cup without hitting the target. The carbon aperture also has a current readout which aids in tuning the beam through the rabbit.

The new compressed air system utilizes shop air ( 100 psi) which is fed through a pressure regulator and a needle valve to a section of 2 " beam tube. The beam tube is used as a reservoir for the compressed air. Between the reservoir and the rabbit tube is a solenoid operator valve which is opened and closed by a timer.

The new vacuum system is similar to the compressed air system. A vacuum cleaner provides suction that is regulated by a needle valve and another 2 " beam tube reservoir. Between the reservoir and the rabbit tube is another solenoid operated valve which is open and closed by the timer. The timer allows the opening and closing times of both valves to be independently adjusted. With this arrangement the compressed alr blows the target from the bombarding site to the counting site and the suction pulls the target back from the counting site to the bombarding site.

By adjusting the regulator and needle valves the transit times for the targets can be adjusted. The time for the rabbit to return from the counting site to bombardment site is about 1 second. This relatively slow time was chosen to extend the life of the balsa wood carriers. The fastest practical transit time for the trip from the bombaring site to the counting site is $80 \mathrm{~ms}$. For the ${ }^{24} \mathrm{Mg}(\mathrm{p}, \mathrm{n}){ }^{24} \mathrm{Al}$. $\quad \beta-\gamma$ circular polarization correlation experiment, the transit time is set at $400 \mathrm{~ms}$. 
4. Dual Carbon Foil Time-of-Flight System

D. Leach and A.G. Seamster

A second fast timing detector for heavy ions has been constructed which incorporates a stack of two microchannel plates as an electron multiplier. The first unit was described in a previous Annual Report. 1 These devices, based on the design of Zebelman et al.., ${ }^{2}$ utilize the isochronous transport of secondary electrons produced when a charged particle passes through a thin carbon foil. The transport is accomplished by crossed electric and magnetic fields.

Fast preamplifiers have been built using recently available high speed hybrid circuits that allow retention of rise times down to 1 nsec:

A time resolution of $200 \pm 25 \mathrm{psec}$ has been measured for $5.48 \mathrm{MeV}$ alpha particles using the dual carbon foil system. Ihis indicates a resolution of 141 $\pm 18 \mathrm{psec}$ for each unit. This value should improve with more energetic, heavier ions.

\section{References}

1. Nuclear Physics Laboratory Annual Report, University of Washington (1978), p. 124 .

2. A.M. Zebelman et al., Nucl. Instr. and Meth. 141, 439 (1977).

\section{Th-228 Alpha Source Generator System}

K.T. Lesko and A.G. Seamster

A new $10 \mathrm{mC}$ Th-228 alpha source generating system has been installed in the radiochemistry lab. Al pha sources are produced by the deposition of $\mathrm{Pb}-212$ onto needles or foils. The resultant sources produce alpha particles with principal energies of $6.051 \mathrm{MeV}, 6.090 \mathrm{MeV}$, and $8.785 \mathrm{MeV}$.

The Th-228 bearing compound is housed in a specially designed, normally sealed, stainless steel and lead pig, which is enclosed in an air-tight fiberglass glove box equipped with an air lock.

6. Design of a Large-Solid-Angle Isochronous Momentum Filter

K.J. Davis, R.A. Loveman and D.W. Storm

The design for the momentum filter/spectrograph that was reported in last year's annual report ${ }^{1}$ has been changed substantially. The design goals are the same, namely to build a mirror symetric system so that at the final image there 
will be an achromatic, isochronous focus of small enough size to use a solid state detector for energy determination. At the mirror symmetric plane there should be a focus with enough dispersion to give momentum resolution of several times 10-3. Finally, the acceptance solid angle of the device should be reasonably large over a reasonably large energy range. After detailed studies, we determined that the energy range of the device described previously was inadequate. Consequently we have spent a major design effort this year to find a system that would meet all the design goals, including that relating to energy range. The effort was successful, and the new system will be described below. As it is somewhat more expensive than the original, there has been some delay in ordering components. As this report goes to press the additional funds have been granted, and we can commence the construction phase.

While the previously described system consisted of a quadrupole magnet, a $60^{\circ}$ bending magnet, and another large bore quadrupole magnet before the symmetry plane, the new system retains the first quadrupole, then has a $45^{\circ}$ bending magnet, followed by another magnet bending 45 in the other direction. In each case there is a mirror symmetric duplication of the first three magnets after the symmetry plane. The new configuration is shown in Fig. 9.6-1. In both cases there is a double focus at the symmetry plane, which must be perpendicular to the central ray, and the elements are chosen so that central rays of momentum differing from the central momentum are parallel to the central momentum ray at the symmetry plane. These four requirements are necessary to obtain mirror symmetry, which, in turn, guarantees achromaticity and isochronism at the final focus. In both systems second order corrections are required in the third element in order to make the focal plane perpendicular to the central ray.

The new configuration is described in Table 9.6-1. In the previous system, the second quadrupole magnet was used to make central rays of all momenta parallel. Thus it was horizontally focusing. In the present system, the reverse bending of the second dipole magnet accomplishes that task, and so it need not be horizontally focusing. In fact it was found that the best vertical acceptance was obtained if that magnet was vertically focusing and was neither focusing nor defocusing in the horizontal plane. In either system, the achromaticity applies to the horizontal plane, but not the vertical plane. It would be possible in principle to make the vertical focus achromatic, but we found that the second order corrections required to achieve vertical achromaticlty tend to counteract those that are necessary to make the mirror symmetric focal plane perpendicular. In order to achieve both these requirements, several huge second order corrections were required in various magnets. The net result was that the system could be made doubly achromatic to first order, but higher order chromaticity was then enormous, and the focusing aberrations were also very big. Consequently we did not pursue the attempts to achieve double achromaticity further. 
Table 9.6-1: Magnets for momentum filter

Quadrupole

$\mathrm{L}_{\text {eff }}=20.0 \mathrm{~cm}$
radius $=5.0 \mathrm{~cm}$
gradient $=1.3 \mathrm{~kg} / \mathrm{cm}$ for $25 \mathrm{MeV}$-Amu

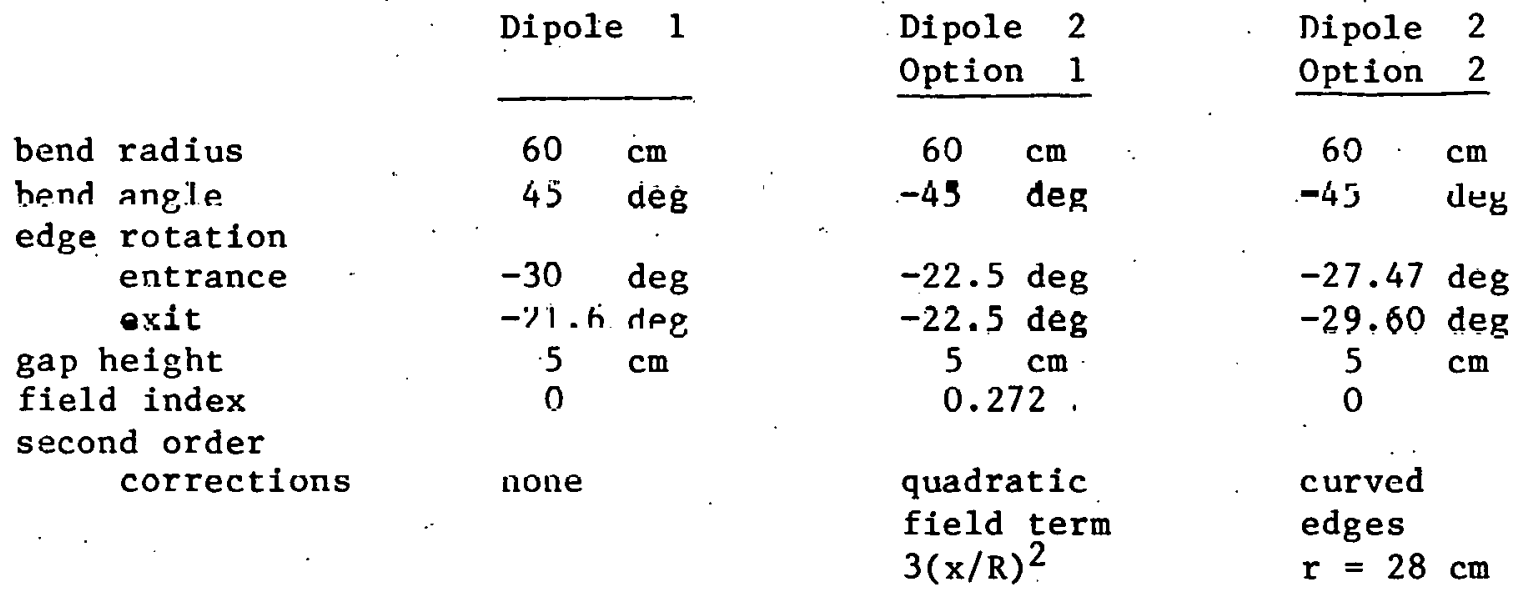

NOTE: Negative rotation angles for Dipole 2 correspond to vertical focusing, since the magnet bends opposite the conventional direction.

Fig. 9.6-1: Scale layout of the first half of the mirror symmetric system. The magnet effective field areas, but not the yokes are shown. Three rays are illustrated: the central ray, a central angle ray with $10 \%$ higher momentum, and a large angle central momentum ray.

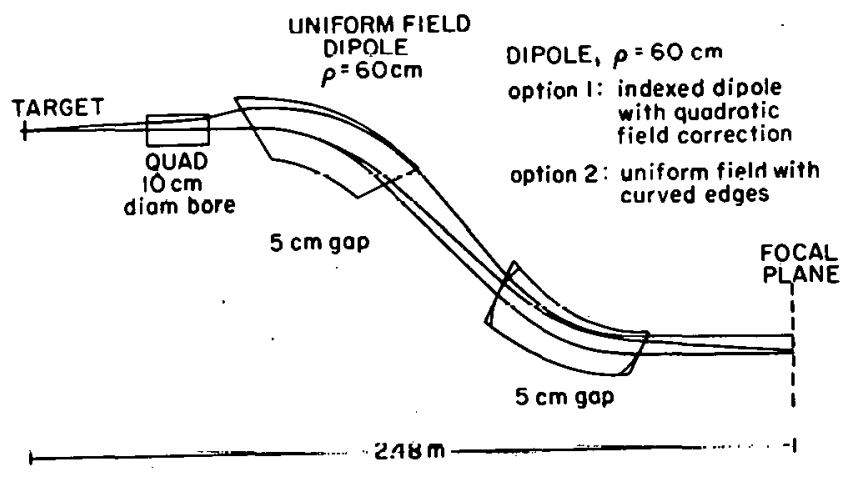


However, we found that by combining the vertical focusing of the second dipole with that of the first quadrupole, we had the vertical focusing distributed along the system well enough that the vertical acceptance was quite independent of momentum. Basically what happens is that even though off-momentum particles will be focused to an image quite far from the mirror symmetry plane, as soon as they get very far from the central axis they encounter strong vertical focusing and are forced back towards the axis. In the previous system, nearly all the vertical focusing was in the first quadrupole, and there was net vertical defocusing in the other magnets. Consequently off-momentum particles followed trajectories that were bent too much or too little in the first magnet and then drifted a long distance. Thus small variations from the correct angle of the first bend (corresponding to small variations in momentum. from the central momentum) eventually placed the particle far from the system axis.

The optical properties of the two systems are listed in Table 9.6-2. Although the new system has poorer first order resolution, for large solid angles the resolutions of the two systems are simflar. Since most applications of the device will use a solid state detector to determine energy, resolution is not particularly critical. The other major difference in the two systems is in expense. Although we are replacing a $60^{\circ}$ bending magnet with a $45^{\circ}$ one, that saving is not sufficient to cover the additional cost of the second dipole over the second quadrupole, even though that quadrupole was to have a large bore. Thus the magnets of the new system cost somewhat more than those for the previous system.

We have done a number of ray-tracing calculations to determine the properties of the system for various solid angles and for central and non-central momenta. The main results of these calculations are presented in terms of the spot size at the final focus, the resolution as a function of solid angle, and the solid angle acceptance as a function of momentum. These quantities are illustrated in Figs. 9.6-2 through -4 . The ray-trace calculations were carried out using a computer program written by Enge, Spencer, and Kowalski.2 They use magnetic fields that are parameterized in terms of the shape of the fringe field and the multipolarity (for quadrupole magnets) or the polynomial expansion of the mid plane field (for dipole magnets). The fields we have used correspond to clamped fringe fields. The second order corrections in the second dipole are done either with curved edges or with a midplane field expansion that has a second order term in the $x$ cooordinate (perpendicular to the beam and in the bend plane). In addition, the focusing of that magnet can be done either with rotated edges or with a field index. The first dipole magnet requires a third order correction, which can be carried out by curving the entrance edge. The curve is a pure cubic, and its magnitude is such that the deviation from a straight line is $1.4 \mathrm{~mm}$ at a distance of $10 \mathrm{~cm}$ from the entrance of the central ray. Enge has suggested that such a correction can be built by building the magnet with a removable insert along the edge. Then by cutting the insert the field can be weakened to give the same effect as that of a curved edge. 


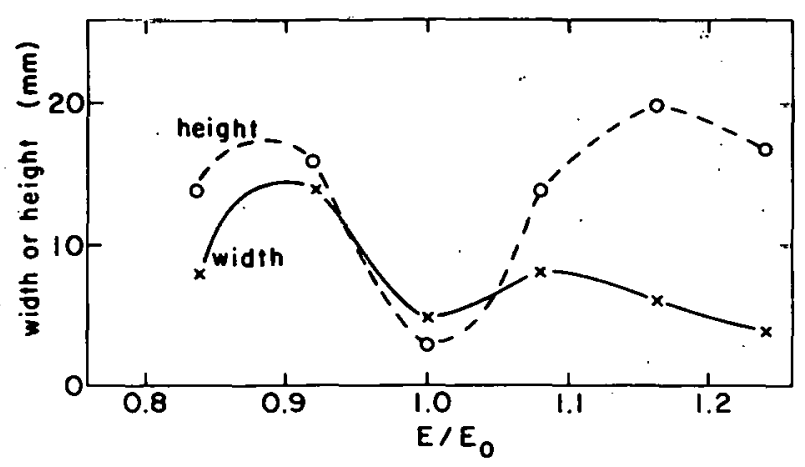

Fig. 9.6-2: Final spot size dimensions as a lunction of $\mathbb{E}^{\prime} \mathrm{E}_{\frac{1}{}}$ for the full. soiid angle.

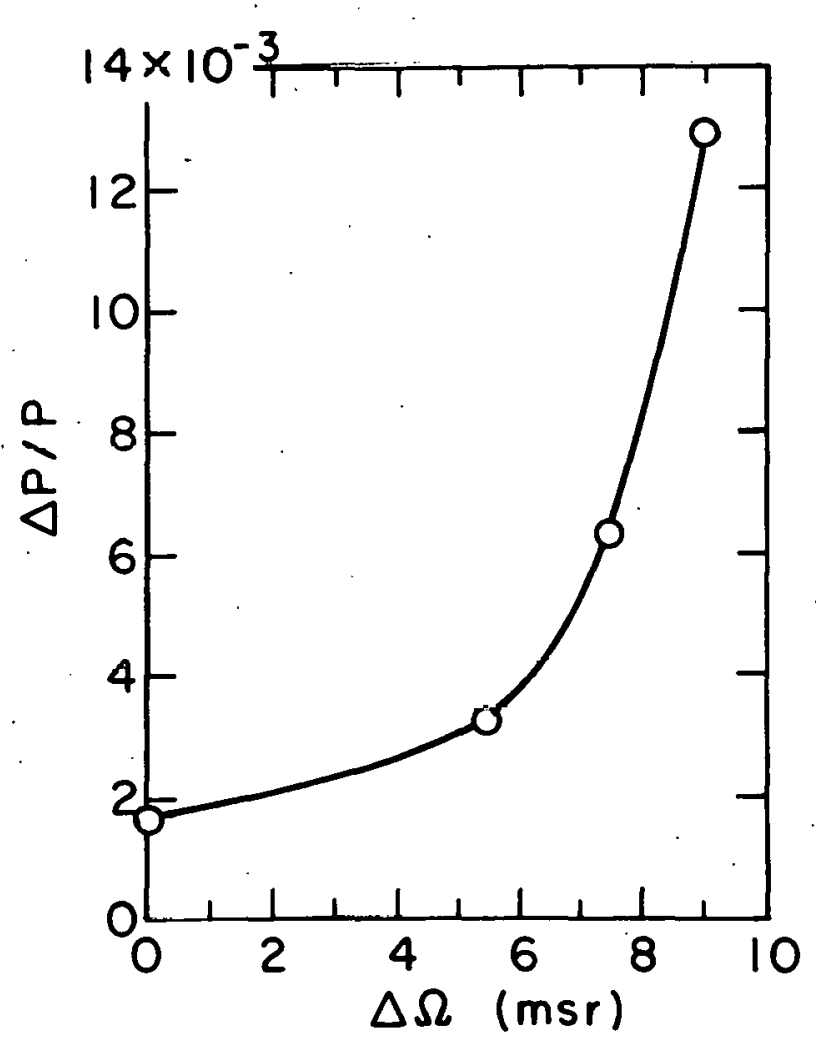

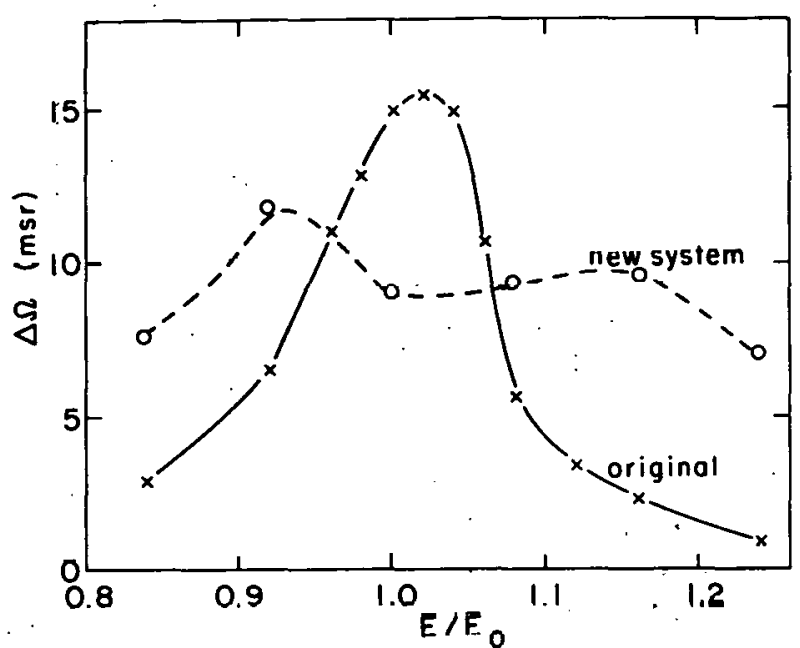

F1g. 'y.6-j: Solld angle vs $E / E_{O}$ for new system (dashed lines) and compared to the system as described last year.
Fig. 9.6-4: Rcsolution vs. $\Delta \Omega$, at the momentum central energy. 
Table 9.6-2: Comparison of optical properties of new and old system

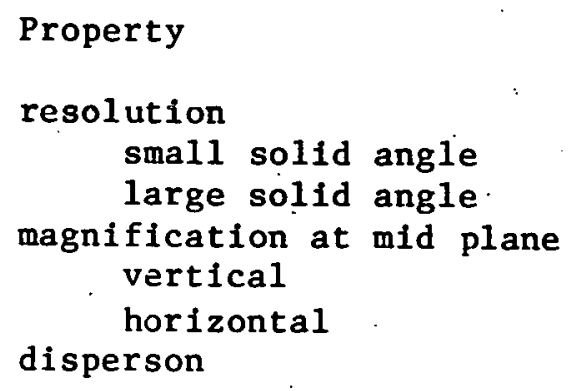

\author{
New System \\ 01d System
}

$0.1 .4 \%$

$1 \%$

$0.09 \%$

$1 \%$

$\begin{array}{ll}2.1 \% & 6.1 \% \\ 0.9 \% & 0.4 \% \\ 0.66 / \mathrm{cm} / \% & 0.7 \mathrm{~cm} / \%\end{array}$

\section{References}

1. Nuclear Physics Laboratory Annual Report, University of Washington (1979), p. 146 .

2. J.E. Spender and H.A. Enge, Nucl. Instr. and Meth. 49, 181 (1967); and S. Kowalsi, private communication. 


\section{Tandem Energy Controller System}

\section{H. Fauska, T.A. Trainor, and C. Wagner}

During the past year construction of a new energy controller system ${ }^{1}$ was completed. In the process of installing this unit we closely examined all components of the energy regulation system and made a number of modifications as discussed below. The result is a beam energy spread less than $300 \mathrm{eV}$ over most of the tandem voltage range of 1-9 MV and with a range of image slit currents from less than $1 \mathrm{nA}$ to $1 \mu \mathrm{A}$ or more.

Each major component of the regulation system is described in detail below.

Image Slj.ts and Preamplifiers

This oyotem, as it existed, suffered from the following faults: (1) the air cooling for the slits produced acoustical and electrlcal uvise, (2) holos in the slit housings which passed the coollug lines aloo permitted capacitive coupling to the environment, and large AC noise currents were generated; (3) multiple ground points throughout the control system formed further AC noise sources, (4) cables from slits to preamps were microphonic and picked up vibrations from mechanical pumps, (5) the $\log$ preamp frequency response at low currents was poor $(0.1-1$ sec time constant for $I<1 \mathrm{nA})$, and (6) the slits were directly opposite one another and unsuppressed so that the relationship between beam position and slit currents, especially for heavy ion beams, was ill defined. In fact, for much of the time the slit currents were actually opposite in polarity to the primary beam current. This was tolerated by the $\log$ preamps only because the feedback path was reversed parallel diodes, which permitted bipolar operation but severely degraded the uniformity of the log characteristics.

The remedies were as follows: (1) a closed oil circulation system was installed to cool the slits. The noise generated by the oil is much less than for air cooling and corresponds to a noise curreut of -50-100 p 4 . The conting system is turned on when power deposited on each siit exeeds 10 watts; (2) shrouds were constructed to fit over the slit housings and greatly reduce the effective aperture for couplng to external noise sources; (3) proper grounding techniques were applied to the whole regulation system. Optical isolators were used immediately after the log preamps; (4) low-noise cables (graphite-coated dielectric) were installed between slits and $\log _{\text {preamps; (5) after first }}$ trying some commercial hybrid $10 g$ preamplifiers ${ }^{2}$ which were expensive, failed often, and also had poor frequency response at low slit currents, we adapted a more sophisticated design ${ }^{3}$ which includes temperature compensation and, most important, active compensation of the transdiode capacitance in the log amp feedback path. This compensatiun permits flal frequency response to $1 \mathrm{kHz}$ for slit currents less than $1 \mathrm{nA}$; and (6) the slits were staggered ( 8 " apart) and enclosed in separate suppression cages within the drift tube. The low energy slit is further downstream. 
The performance of the log preamps is excellent. In addition to the good frequency response mentioned above the log characteristic is very uniform (to less than $1 \%$ ) over a range from $10 \mathrm{pA}$ to more than $100 \mu \mathrm{A}$. The output is 1 volt/decade, and a bias current of $30 \mathrm{pA}$ is inserted to establish a zero volage reference and prevent electronic saturation when the beam is interrupted.

Generating Voltmeter (GVM)

The details of this device have been reported previously. 4 The only modification made recently has been removal of a low-pass filter in the output which was intended to attenuate a $280 \mathrm{~Hz}$ ripple associated with the rotor action. The filter had the undesirable effect of introducing an additional phase shift over a useful range of frequencies, thus reducing the maximum stable gain in the corona triode regulation loop when in GVM control.

Corona Triode Regulator

The corona triode (6BK4) was found to have an improper quiescent grid voltage $(-1.5 \mathrm{~V})$ so that it almost always clipped the error signal. It was reset to $-3.5 \mathrm{~V}$, which provides for a considerable margin over maximum error signal variations ( $\pm 2 \mathrm{~V}$ typically).

The frequency response of the triode regulator was measured over a frequency. range from $1 \mathrm{~Hz}$ to about $150 \mathrm{~Hz}$. The response was consistent with a single low-pass RC stage (terminal-to-tank $C$ and column $R$ ) in conjunction with the effect of an ion time-of-flight of $-30 \mathrm{~ms}$, which produces a sharp cutoff in the response at about $30 \mathrm{~Hz}$. In addition, maxima and minima at higher frequencies were observed as expected.

Terminal Ripple Remover (TRR)

Details of this device have been reported previously. 5 Upon inspection it

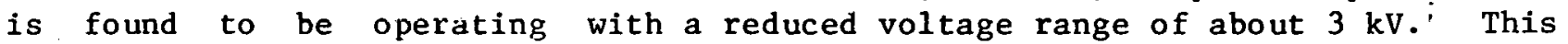
range was inadequate for most tandem operating conditions, and clipping resulted. It was extended to about $4.5 \mathrm{kV}$ by increasing the supply voltage and modifying the phototransistor receiver circuit. A satisfactory range would be 6 $\mathrm{kV}$ or greater.

The frequency respose of the TRR was measured and found to be equivalent to two low-pass RC stages with corner frequencies at $450 \mathrm{~Hz}$ and $2.5 \mathrm{kHz}$. This response indicates that a maximum stable gain of only about 6 was permitted in the TRR loop. In addition, the lack of an effective high pass stage in the TRR meant that this unit was "competing" with the corona loop to correct DC terminal drifts of hundreds of kilovolts, driving it into saturation periodically.

A filter was introduced into the TRR loop which moved the first low-pass corner frequency from $450 \mathrm{~Hz}$ down to $30 \mathrm{~Hz}$ and added a high-pass stage with corner at $2 \mathrm{~Hz}$. This gave a response which peaks over the charging belt fundamental $(2.5 \mathrm{~Hz})$ and lowest few harmonics and has a maximum stable gain of about 100 . 
Another limiting factor in the TRR is the presence of $800 \mathrm{~Hz}$ ripple from the high-voltage power supply. This ripple is about $20 \mathrm{VP}-\mathrm{P}$ and requires a substantial phase margin to avoid oscillation at $800 \mathrm{~Hz}$. This is now the principle noise source in the regulation system and reduces the maximum stable gain in the TRR by a factor of $2-3$.

Energy Controller

This unit is installed in the tandem control console. It contains the gain adjustments for the two feedback loops (corona triode and TRR), the quiescent current adjustment for the TRR LED (this determines the DC voltage value on the terminal stripper), the GVM voltage setpoint adjustment, and switching elements which determine whether each of the two loops receives an energy error signal from the image slits or from the GVM. Panel meters display separate slit currents ( $\log$ scale) for each of the object (horizontal and vertical) and image (horizontal only) slits plus error signals from the lmage slits and GVM.

An auto mode is available in which the corona loop switches from image slits to GVM automatically if the total image slit current drops below a threshold set on the front panel. This is very desirable for quick recovery from tank sparks and beam interruptions, among other things.

Another feature connected with the auto mode which is planned but not yet implemented involves forcing the corona loop to remain in GVM control until the GVM value is within a preset voltage window. The loop is then released to slit control if sufficient beam is present. This will solve a problem which exists with heavy ion beams in which, following a tank spark, the machine goes into slit control on an unwanted, but more intense, charge state at lower terminal voltage. This condition cannot be prevented with the slit current threshold alone.

Criteria for adustment of the various conrols are fairly simple. Belt charge is increased until the corona current reaches $50-60 \mu \mathrm{A}$. Corona points position is adjusted so that the average difference signal from GVM or slits is zero. The gains of corona and TRR loops are raised until oscillation is incipient in both loops ( $800 \mathrm{~Hz}$ for $T R R, 30 \mathrm{~Hz}$ for corona). This proces is iterated a few times until the system performance is peaked. The resulting image slit error signal is usually 50-100 mV P-P, $800 \mathrm{~Hz}$ noise, which is about three times the slit preamp noise with no beam.

\section{References}

1. Nuclear Physics Laboratory Annual Report, University of Washingtnn (1979), p. 156 .

2. Analog Devices Model $757 \mathrm{~N}$.

3. C.A. Grimbergen and G.H.P. Konnke, Rev. Sci. Instr. 47, 854 (1976).

4. Nuclear Physics Laboratory Annual Report, University of Washington (1978), p. 119 .

5. Nuclear Physics Laboratory Annual Report, University of Washington (1973), p. 2 . 
8. Design and Construction of Electronic Equipment

M. Bizak, H. Fauska, R.E. Stowe11, T. Van Wechel, and C.L. Wagner

The 200 megahertz analog-to-digital converters have been interfaced to the new computer (see Sec. 10.2, this report). The installation involved designing and constructing signal handling and conditioning equipment to provide singles or coincidence operation as well as routing capabilities. The coupling electronics is completed and functioning. Provision to start and stop data accumulation was provided in coupler circuits.

Sixteen of the new ten digit scalers have been installed and working with the new computer. The interface electronics were designed and constructed (see Sec. 10.2).

The electronics associated with the position sensing proportional counters (see Sec. 9.1) were designed and constructed. The items are similar to circuits used at Heidelberg and include the following:

1) One four channel high voltage control to sense any voltage sparks and disconnect the common high voltage supply.

2) Two noninventing pre-amplfiers with six channels per double width NIM module.

3) Two preamplifier units similar to item 2) with inverting outputs on four of the six channels.

4) One six channel. booster high frequency amplifier to provide the proper level signals for certain types of counter usage.

The design and construction of the energy control circuitry is described in Sec. 9.7 .

The 200 megahertz analog-to-digital converters required considerable maintenance during the transition from the old computer to the new computer as experimenters would tend to change locations. The need to provide faster repair prompted us to design and construct an exerciser to test them.

The need of only allowing very small deviations in energy on experiments running with no readable image slit currents required the construction of a circuit to sense the digital voltmeter reading the generating voltmeter and if it fell outside of a digital set window to automatically put in the high energy Faraday cup.

Work performed with cancer research funding to upgrade the cyclotron reliability is described in Sec. 8.3. 


\section{G. Hinn}

The targets listed below have been prepared in the target lab over the last year. Of the 200 targets prepared only a few of the more interesting and non-standard techniques will be briefly described in detail.

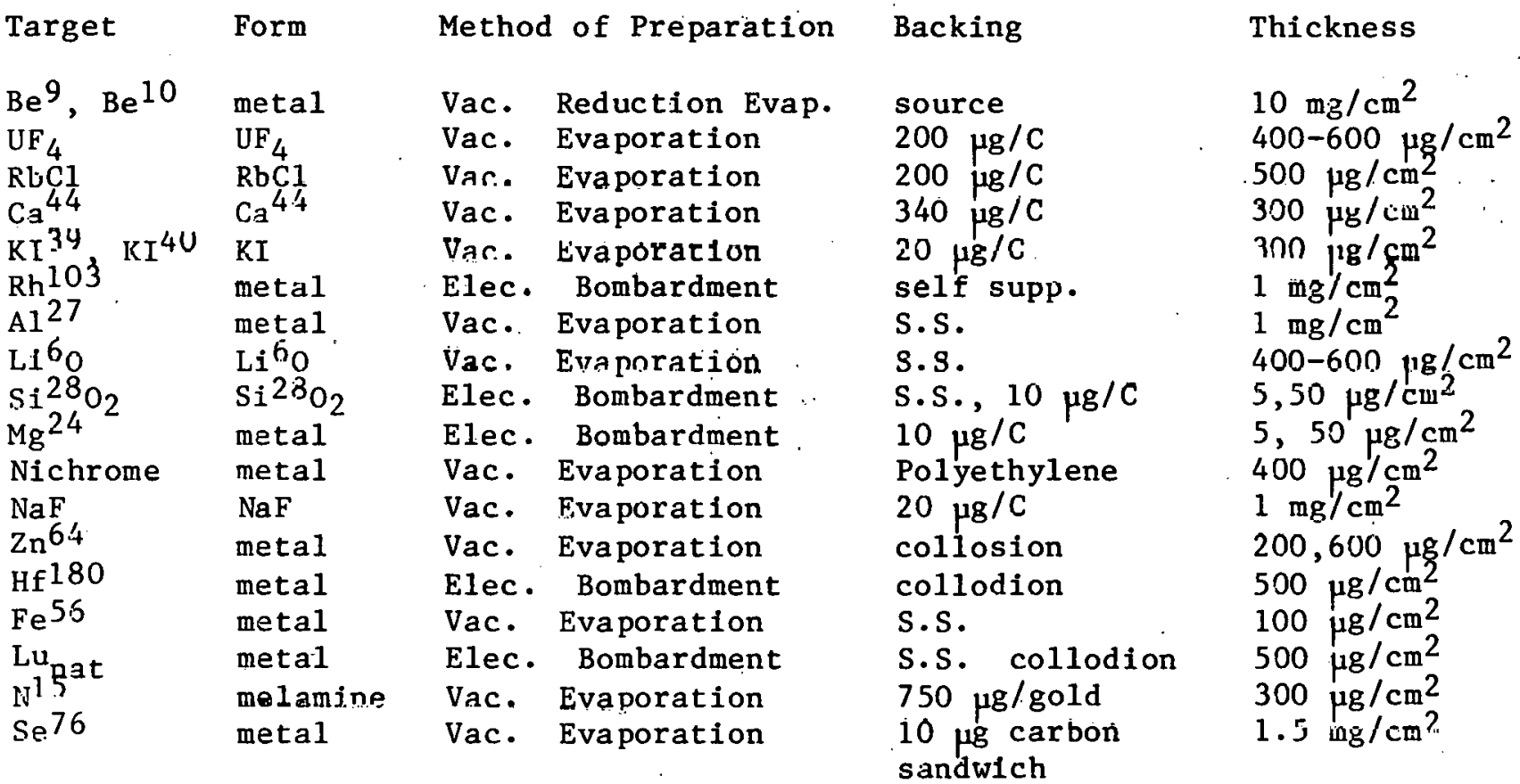

\section{a) Nitrogen}

Solid natural and $\mathrm{N}^{15}$ melamine $\left(\mathrm{C}_{3} \mathrm{H}_{6} \mathrm{~N}_{6}{ }^{15}\right)$ targets were successfully made on thick gold backings. $\mathrm{N}^{15}$ labelled melamine has been unavailable commercially over the last ten years but is now obtainable from a small supplier (Isotope Labeling Corp., 622-D Route 10, Whippany, NJ 07981). It has a higher nitrogen content than Adenine $\left(\mathrm{C}_{6} \mathrm{H}_{5} \mathrm{~N}_{5}{ }^{15}\right)$ and is easily resistance evaporated, M.P. lcss than $300^{\circ} \mathrm{C}$.

\section{b) Beryllium}

Successful beryllium source pellels were made in the beryllium pvaporator. 'l'his $1 \mathrm{~s}$ all evaporator enclused in g glove box system. The oxide was reduced using magnesium as the reductant in a small tantalum crucible which was heated in a Westgaard and Bjornholm axial type electron gun. 
The entire beryllium evaporator system is presently undergoing an upgrading and expansion with the addition of a joining glove box where weighing and sintering can take place. Most of the work in this system will be beryllium accelerator dating oriented.

\section{c) Hafnium}

Due to the difficulty of rolling hafnium metal, a method was devised whereby an electron gun was used to melt a hafnium pellet on a tungsten boat to the desired thickness $(10 \mathrm{mil})$. Thin $\left(200-300 \mu \mathrm{g} / \mathrm{cm}^{2}\right)$ hafnium 180 targets were made by evaporating the metal from a graphite crucible in a Westgaard and Bjornholm axial type electron gun. To prevent curling upon floating in water, the hafnium and potassium iodide substrate coated glass slide were first imnersed in a collodion solution ( $10 \%$ collodion and $90 \%$ amyl acetate).

\section{d) Stripper Foils}

The tandem Van de Graaff 40 position foil wheel assembly has been replaced 3 times in the past year. Commercially prepared (Arizona Carbon Foil Co) 3-10 $\mu \mathrm{g} / \mathrm{cm}^{2}$ carbon foils were installed..

Current1y, collodion (cellulose nitrate $\left(\mathrm{C}_{12} \mathrm{H}_{17} \mathrm{~N}_{3} \mathrm{O}_{16}\right) \mathrm{X}$ backed 3-10 $\mu \mathrm{g} / \mathrm{cm}$ carbon foils are being tested. Collodion backing on thin carbon foils resulted in a great savings in money and time in mounting the foils in the target wheel. Normally a foil change without collodion requires 3 days, with collodion the change takes $1-1 / 2$ days with a financial savings of $\$ 200$ per foil change.

Collodion has also been used as a backing for targets other than the stripper foils with no interference since it burns off in the beam. One case only has been reported where it did not burn off. In this case a $50 \mathrm{MeV}, 40$ nanoamp carbon beam was used on a collodion backed carbon-13 target.

\section{Compton Polarimeter Switching Bridge Using High Power FETS}

\section{Peterson and H.E. Swansun}

Dperation of compton polarimeter requires magnitizing an iron absorber (or scatterer) with one direction of flux, counting the transmitted $y$-rays for that state, then reversing the flux direction and counting the $\gamma^{-} s$ for the reversed state. Instrumental asymmetries due to magneto-striction, etc., are greatly reduced if the two saturation fluxes are opposite but equal in magnitude. This is easily achieved if a unipolar current source is used to power the magnet through a solid state switching bridge or reversing switch. The circuit configuration is simply that of a bridge rectifier where the diodes are replaced by switching elements, in this case newly available high power FETS. The power supply is connected to one pair of opposite nodes of the bridge and the magnet 
coil to the other. By alternately energizing opposite pairs of FETS the coil current and thus the saturation flux can be reversed preciscly. The current source sees at most a slightly different resistance in each $O N$ state and its current regulation is uneffected.

The FETS used in our design (IRF 331) were made by International Rectifier Corporation, although other companies now make similar devices (e.g., Siliconix, Inc.). These have ratings of 350 volts and $4 \mathrm{amps}$ and as they are operated either turned on or off, and switch in nanoseconds, power dissipation is otherwise not a problem. FETS are easily paralleled for increased current capacity and require simpler drive electronics than do transistors.

The unit is used with an OP Amp power supply such as the KEPCO OPS series, for its current source: These have negliglule output capacitance and can thus rapidly respond to correct the current error upon field reversing. A large part of the switching time is due to eddy curreuts generated in the magnet itself. These currents oppose the currents in the coils, and die away in a time characteristic of the resistivity of the material. In an attempt to cancel these currents and thus speed up the switching time, an exponentially decaying signal is added that coming from the current sense resistor. This causes the current following a transition to remain higher than that required for steady state saturation and then to fall off such as to compensate for the eddy currents.

An integrator is included in the chassis, which is used along with a sense coil would around the magnet core, to observe the magnetic flux in the iron.

This circuit will be used to power a lew 4 fold polarimerer using 5" $x$ 6" NaI detectors. The polarimeter is being constructed at Chalk River for use in a new measurement of parity mixing in $21_{\mathrm{Ne}}$.

11. A Polarimeter for Measuring Proton Beam Polarization

H.C. Bhang, S. Laubach, * T.A. Trainor, and W.G. Weitkamp

For the measurements of depolarization in proton inelastic scattering from copper described in Sec. 3.5 of this report, it is necessary to measure the polarization of an $18 \mathrm{MeV}$ incident beam. To accomplish this, we have constructed a special beam polarimeter using elastic scattering from carbon as the analyzier.

Previous measurements of the analyzing power for elastic scattering of protons from carbon show that the largest analyzing power occurs at $\theta_{1 \mathrm{ab}}=$ $145^{\circ} .1$ our polarimeter holds two detectors at this angle, one 1 eft and one right of the beam, clamped to the fixture in the 60" scattering chamber containing the beam defining apertures, upstream from the copper target. Detector apertures are $1.1 \mathrm{~cm}$ in diameter, $10.5 \mathrm{~cm}$ from the $20 \mu \mathrm{g} / \mathrm{cm}^{2}$ carbon target. This carbon thickness gives good statistical precision but interfers negligibly with beam energy or angle spread. 
Fig. 9.11-1: Polarimeter analyzing power. Open circles are measurements of Ref. 1 ; closed circles are the present measurements.

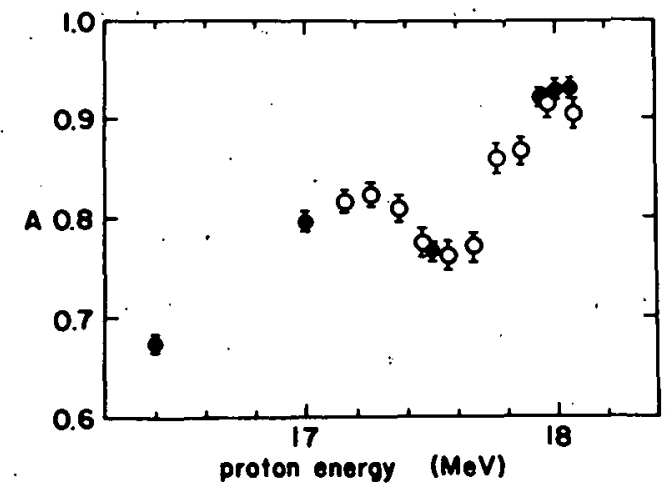

The polarimeter has been calibrated by comparing its analyzing power with that of proton scattered from helium at $112^{\circ} .^{2}$ The analyzing power of the polarimeter in the energy region where it is large is shown in Fig. 9.11-1: The maximum value of $A$ is $0.929 \pm 0.011$ at $18.0 \mathrm{MeV}$.

\section{$\underline{\text { References }}$}

* Department of Physics, University of Washington; visiting from Darmstadt.

1. H.O. Meyer, W.G. Weitkamp, J.S. Dunham, T.A. Trainor, and M.P. Baker, Nucl. Phys. A269, 269 (1976).

2. P. Schwandt, T.B. Clegg, and W. Haeber1i, Nucl. Phys. Al63, 432 (1971).

\section{Resistive Film Position Sensitive Detector}

Don Leach

Based on a design by Jared et al, a two-dimension position sensitive detector is under construction. The detector is a parallel-plate gas detector with NiCr wiudows on a polypropylene backing. Position is determined by charge division of secondary electrons across a $\mathrm{NiCr}$ film.

The NiCr films are produced by evaporation onto polypropylene. During the evaporation the plastic is backed by a copper sheet cooled with liquid nitrogen.

Initial tests with $72 \mathrm{MeV} 160$ on the partially completed detector were encouraging but not conclusive.

\section{$\underline{\text { References }}$}

1. R.C. Jared et al, Nucl. Instr. and Meth. 150, 597 (1978). 


\section{Refrigeration Unit for Chamber Cold Traps}

D. Leach and A. Lazzarini

A portable unit designed to replace liquid nitrogen to cool vapor traps in vacuum systems has been constructed.

The unit uses a heat exchanger with a freon primary $100 \mathrm{p}$ and a secondary cooling loop of a methanol/water solution. This secondary cooling solution can be circulated in the vacuum chamber for whatever apparatus is desired.

The temperature sensing and control circuitry was based on a design described in "Designing with Operational Amplifiers." This approach uses a differential transistor pair biased with unbalanced currents ao the methnd of temperature sensing.

A zero load temperature of $-23^{\circ} \mathrm{C}$ is typical. Under normal workluy loads tempcratures of -16 to $-18^{\circ} \mathrm{C}$ die expcctcd.

\section{References}

1. J. Graeme, Designing with Operational Amplifiers: Applications Alternatives, $254-256$ (1977). 
10. COMPUTERS AND COMPUTING

1. CAMAC-based Data Acquisition System for the PDP 11/60

H. Fauska, R. Seymour, and T.A. Trainor

Our primary input devices are Tracor Northern $200 \mathrm{MHz}$ ADC's. Their outputs are passed through a Laboratory-designed and built routing box which also assembles a :format". pattern showing which $\mathrm{ADC}^{-}$s received data during a coincidence event. The format pattern and routed ADC signals are passed to thirteen Kinetics 3420 inpist modules. These and a pair of LeCroy $2228 \mathrm{~A}$ octal time-to-digital converters are mounted in a standard. Kinetics CAMAC crate which is connected to the PDP $11 / 60$ by a BiRa MBD11.

The MBD11 is actually another computer, built for the task of intelligently interfacing the CAMAC system to a PDP 11. It was purchased with 1024 words of memory, and an additional $3 \mathrm{kw}$ of Intel 2115A memory was added by the Laboratory. The added memory uses less power than BiRa's 93415's, and costs less.

The MBDIl is loaded with programs which preprocess the event information into $a$ packet which is then directly written into the PDP $11^{\text {'s }}$ memory. The PDP 11 is therefore freer to analyze, record and display the data without being loaded down by having to handle high-frequency interrupts.

Three MBD programs provide most of the data collection services. One (QDA) operates with the MULTI/QDA multiparameter analysis group. We have seen about $50 \%$ overall deadtime at a $5 \mathrm{kHz}$ data rate. The other two programs provide for high data rate singles activity. One ( $\left.7 \mathrm{ADC}^{\prime} \mathrm{s}\right)$ uses the MBD11's channel interrupt facility for fast handling of up to $7 \mathrm{ADC}^{\prime} s$. The other (12ADC $\mathrm{s}^{-}$) uses a slower round-robin status checking algorithin which services all 12 ADC's $^{\prime}$ and the two LeCroys. 7ADC's services an ADC upon receipt of a "conversion done" signal frola the ADC. Since the interrupt hardware assigns priorities based on which CAMAC slot signale, a high dara rate in a high-priority ADC . could theoretically block service to a lower-priority CAMAC slot. 7ADC's can select, read and channel increment an event in 10 microseconds. $12 \mathrm{ADC}^{-} \mathrm{s}$ takes 3 microseconds to check an $A D C$ for an event, and 10 microseconds to service it if one is found. The algoithn also distributes the systen dead time evenly among all $\mathrm{ADC}^{-} \mathrm{s}$. The analysis software is discussed elsewhere in this report (see Sec. 10.3).

Additional information is provided by a bank of fifteen $75 \mathrm{MHz}$ scalers and a frequency meter readout, all built at the Laboratory. These are connected to the PDP 11 via an IEEE -488 bus and interface (see Sec. 10.2). 
M.R. Bizak, H.E. Swanson, and C.L. Wagner

An interface was constructed to allow the PDP $11 / 60$ data acquisition computer to read the scalers previously described in the Nuclear Physics Laboratory Annual Reports for the years 1977 and 1978. These scalers are now operational and have been used for nuclear physics research. The decision had previously been made to have peripheral data communication of this type proceed via the IEEE 488 Bus, so that instruments equipped with the IEEE 488 Bus could be easily incorporated into our data acquisition system. Controller chips exist which handle the IEEE Bus protocol but these are most conveniently used as part of a microproccscor system.

Oul design conoists of a core section general enough to be uced for ather interfaces and a section, specific in this case, to the scalers. lhe core section uses a Motorola MC 6802 microprocessor with sufficient memory and support chips to run Motorola'o MIKBUg 2.0 firmware. This is used for developmental purposes and supplied in a ROM as part number MC 6846 P1. A switch selects between the firmware and a ROM containing the routines written for the normal operation of the interface.

In a typical development scenario, a program to, say, read the bank of scalers is written and assembled in native 6802 code on the H-Atom control computer, 1 and loaded into the RAM memory of the interface. Using MIKBUG 2.0 and a terminal, this program can be run and debugged until it is operational. The desired code is then placed in a ROM, using circuitry built for that purpose, and operation of the 6802 is switched to that ROM. The MIKBUG ROM and associated support chips may now be removed for use in other interface modules thus keeping costs down.

The scalers the:llstlves were organizod around an 8 bil data bus, i.e., pairs of BCD digits. The scaler bank consists of 16 scalers requiring 4 bits for addressing and each scaler has 5 digit pairs, realizable in 3 bits. In addition data may be both written to and read from the scalers necessitating a direction bit. Thus one 16 bit bi-directional I/O port (Motorola MC 6821) and some decoding $\log 1 \mathrm{c}$ is all that is required 5 ) construct the scaler-specific section of the interface as mentioned above.

Remote operation of the 1nterface uses staniard IEEE Bus protocol. The scaler controller is assigned a primary address and functions within the controller are given various secondary addresses. Fresently these functinns include: (a) read all acalers; (b) writc data to a sperific scaler; (c) snapshot all scalers; and (d) load or examine a program in the interface RAM. This later was included for the purpose of trying out new interface routines prior to putting them into a ROM without the need of re-installing the MIKBUG firmware and its associated components.

Numerical data are transmitted one digit at a time in ASCll, and are easily converted to binary using the Fortran "Encode" and "Decode" statements. 
Functions (a) and (b) above are self explanatory. The ability to snapshot (c) was included to make a simultaneous measurement of all peripheral devices. Each controller is first put into the snapshot mode, and then the "GET" command is issued on the interface bus. This causes all scalers and addressed devices to latch their present values to be later read by the read routines.

\section{$\underline{\text { References }}$}

1. Nuclear Physics Laboratory Annual Report, University of Washington (1979), p. 39.

\section{Progress on Singles Data Acquisition}

\section{K. Green, R. Seymour, and K.A. Snover}

The new singles acquisition system has developed over the past year to a fully functional system which has replaced MULTSING and our aging SDS 930 computer. The capabilities of the new system far exceed those of MULTSING, enabling many experiments which could not be performed before due to lack of resolution, memory limits, speed or number of ADCs available. Summarizing in brief :

1. Resolution. We can now take spectra 8192 channels long. This is twice the limit imposed by MULTSING.

2. Speed. The new singles is able to put data away in 10 microseconds, five times the speed of MULTSING.

3. Memory. Data arrays are limited only by available memory. We current1y use approximately 48 Kwords (24 Kchannels). MULTSING could address at most 6 Kchannels.

4. ADCs. The program can handle up to twelve $200 \mathrm{Mhz}$ ADCs through the CAMAC subsystem. This limitation is imposed by the hardware available.

5. Flexibility. The program is highly modular, consisting of a number of largely independent tasks. Improvements and additions can be made, in many cases, while acquisition is in progress.

The program is controlled by typing mnemonic commands at a terminal, followed by command modifiers. If the modifiers are omitted, the user is prompted for any needed information. There is an extensive default capability to minimize the information the user must supply. In many cases, responses are remembered and become the defaults. For example:

>PLOT DELTA/LOG/CHANNEL : $200: 400$ 
produces a $10 \mathrm{~g}$ plot of channels 200 to 400 of spectrun "DELTA". Subsequent plot commands for spectrum "DELTA" will by default be logarithmic plots of channels 200 to 400. The modifiers can usually be abbreviated to a single character. Had no spectrum or modifiers been specified, the user would have been asked for a spectrum, plot mode and ranges.

As mentioned above, the program is highly modular. Each of the memonic commands is a separate program "known" to the RSX-11M operating system. Modifications to a command occur simply by replacing the relevant program, which can be accomplished while acquisition is in progress. New commanis are added in a similar manner. The data the commands operate on resides in a global common.

Data is acquiren from the CAMAC subsystem with a Microprogrammable Branch Driver (MBD). The MBD is an iniependent processor downloaded with a program to read the $A D C s$ and place the resilting conversions in the $P D P 11 / 60$ via direct memory access (DMA). Once started, acquisition continues witlisut furcher intervention from the $1 \mathrm{l} / 60$.

We currently have two MBD programs for singles acquisition. The fastegt version handles $7 \mathrm{ADCs}$, with priority servicing. $\mathrm{ADC} 1$ has the highest priority; $A D C 7$ the lowest. Service time for the highest priority $A D C$ in use is approximately 10 microseconds. Speed is achieved in this code by using hardware features of the MBD which restrict the total number of ADCs that can be handled. A slower code is available for servicing all 12 ADCs in a non-priority "round robin" fashion. Service time for an ADC is approximately 10 microseconds plus an overhead of 3 microseconts per ADC in the round robin loop. This method has the side effect of equalizing the dead times in all the ADCs.

The $11 / 60^{\prime} s$ main duty during acquisition is to produce a live di6play nn a DEC VT-11 crt. The display interacts with the user via a light pen, allowing selection of spectra, scale factors, and peak and background definitions. Peak areas calculated from these definitions appear on the printouts, as do the reailouts from a bank of 16 scalers.

The printouts are in the form of a high density histogram using the plot capability of our PRINTRONIX-300 line printer. The data is also printed numerically next to the histogram. A running sum is also printed to facilitate manual calculation of peak areas. As plotting may take a long time, runs are dunped to an intermediary disc file from which the plot is generated by a specialized spooling program.

Permanent storage of runs is on 9 track 1600 BPI magnetic tapes. 
4. Progress in Multiparameter Acquisition

K. Green, R. Seymour, and R. Vandenbosch

We obtained the initial version of our multiparameter code from Everett Harvey of . Lawrence. Berkeley Laboratories. It consists of the Fermilab MULTI analysis program and the QDA acquisition program which was written by Everett Harvey. Our hardware configurations are sufficiently similar so that only minor modifications were required to run the programs on our equipment. We have since heavily modified both programs to tailor them for our experimental environment.

MULTI is a very general and well developed event analysis. code which is supported on both the RSX-11M and RT-11 operating systems. It possesses a dictionary of user definable variables and is capable of performing arithmetic and/or logical operations on these variables by interpreting symbolic statements in a language very similar to fortran or basic.

The results of analysis are displayable as one-dimensional histograms or two-dimensional scatter plots on a TEKTRONIX 4006 storage scope. Hard copy of the TEKTRONIX images is produced directly on a PRINTRONIX-300 lineprinter by a TRILOG printer adapter board.

MULTI was modified to store two-dimensional spectra in memory. Prior to this, only a single scatter plot could be accumulated on the storage scope. The amount of memory for this storage is currently limited to about 8.5 Kwords. We plan to expand this to 32 Kwords by placing the data in memory remote from the program.

MULTI has been further modified to control the acquisition code (QDA). QDA is no longer directly visible to, or of any concern of, the experimenter. As it originally came, QDA required a separate terminal to control acquisition.

Timings of the program indicate that it can take 2 parameter data and $10 g$ it on tape at approximately $13 \mathrm{kHz}$. 12 parameter data can be collected at 2 $\mathrm{kHz}$. On-line analysis by MULTI is much slower. MULTI can analyze events at a rate of about $1100 \mathrm{~Hz}$. At higher event rates only a sample of the data is analyzed. This is sufflclent for guiding the user during the experiment. Complete analysis of the data must be performed off line on the VAX 11/780. 
5. VAX $11 / 780$ off-Line Computer System

R. Seymour and T.A. Trainor

Last year we reported the planned purchase of a DEC VAX 11780 computer. It was installed in July 1979 and has taken over the $1 \mathrm{ab}^{\prime}$ s number-crunching. Our current configuration (1isted below) supports 8 interactive and 3 batch users simultaneously. Each user is given 25 kilobytes of memory to work in, with a "virtual" limit of 5 megabytes. When programs access something beyond their 25 $\mathrm{kb}$ limit, the VMS operating system swaps 512 bytes between memory and the disk. This allows each user to write programs as if they had a 5 megabyte machine available.

When the PDP $11 / 60$ was installed, we. saw about an order of magnitude increase in computing "throughput" compared to the SDS $930^{\circ} \mathrm{s}$. The VAX shows a factor of 2 to 10 times better than that of the $11 / 60$.

Among the selection criteria for the VAX was compatibility with the 11/60. This allows us to write and debug code on the VAX which we can then transfer to the 11/60 via magtape. This feature has performed far in excess of our expectations. Executable files (tasks) created on the 11/60 run on the VAX (faster!), and programs which use core-resident libraries and common areas in the $11 / 60$ run on the VAX by automatically accessing disk files instead of the non-existent in-core areas. All editing, compiling and some initial testing of the data collection software is now done on the VAX.

A second selection factor was the need to randomly access very large arrays during data sorting and analysis. People are now running programs with ten 128 by 128 channel data arrays in-program (one megabyte). The 5 megabyte limit mentioned above can be increased, limited only by swapping space on the system disk.

We had intended to move a 67 megabyte DEC RM02 disk from the 11/60 to the VAX. It contained all of the general user files while the $11 / 60$ was the interim off-line computer. During our search for a software "driver" for the VAX (DEC does not support the RM02 on the VAX), we came across an offer which we didn't refuse. We traded in our RMO2, along with $\$ 8000$, and received a 300 megabyte CDC drive with a System Industries controller. This has been installed on the VAX (which revealed a defective VAX Unibus Adapter Board), and the user files were (selectively) moved to it. In the 3 months that the disk has been on-line, the users have managed to occupy the equivalent of $2 \mathrm{RMO2}^{-} \mathrm{s}$, which doesn' $t$ quite use half of the available space on the new disk.

The University's Chemistry Department purchased a VAX and we joined with them for a quantity. discount on Trendata memory boards. We added a half-megabyte of memory, thereby tripling our physical user space. This allowed u.s to double the allocated memory space per user, which should double the effective processing speed. 


\section{off-Line Data Analysis}

DEC VAX $11 / 780$ computer

$8 \mathrm{k}$ byte cache memory

$1024 \mathrm{k}$ byte main memory (Error-correcting MOS)

Two 28 megabyte RK07 disks

One 300 megabyte CDC disk drive with Systems Industries controller

Printronix $3001 \mathrm{pm}$ printer/plotter

Two 9 track 75 ips 1600 bpi tape drives (Pertec)

One 7 track 75 ips 800 bpi tape drive (Pertec)

16 terminal ports

LA36 termina1

Qume 5/55 terminal

Ten Hazeltine 1500 terminals

One Tektronix 4010 graphic display terminal

One Tektronix 4006 graphic display terminal

\section{Three-Body Trajectory Calculation}

W.G. Lynch, M.B. Tsang, and R. Vandenbosch

The exit channel for coincidence experiments like the ${ }^{27} \mathrm{Al}\left({ }^{16} \mathrm{O}_{0},{ }^{12} \mathrm{C} \alpha\right)^{27} \mathrm{Al}$ system discussed in Sec. 6.7 consists of an alpha particle and two other heavier residual fragments. A program call 3FORCE has been written to study the dynamics of these three particles in the combined Coulomb and nuclear field. In the initial state, two of the three particles are in a bound state of their interparticle potentials.

A. Description of the Present Three Body calculation

In general, the calculation is divided into three parts:

1. At $t=0, i . e$. when the calculation starts, two heavy fragments, $27 \mathrm{AI}$ and $16_{0}$ are assumed.

2. At $t=t g$, the relative distance between $16_{0}$ and $27 \mathrm{Al}$ is 15 fm apart. The $16_{0}$ nucleus is represented by a bound system consisting of a ${ }^{12} \mathrm{C}$ and an $\alpha$ particle.

3. For $t>t_{0}$, the trajectories of all three particles are calculated by numerically integrating Newton's equations of motions.

4. The three-body calculation is terminated when the carbon particle has moved more than $100 \mathrm{fm}$ from the point where the $16_{0}$ was first described as a ${ }^{12} \mathrm{C}, \alpha$ bound system. 
Two different forms of nuclear potential have been used: the Woods-Saxon potential and the proximity potentiall.

Both linear and angular momentum are conserved in the calculation independent of integration step size. However, total energy conservation is strongly dependent on the integration step size. Energy conservation is very important in this type of calculation since energy non-conservation often leads to dissociation of the alpha particle from the original bound $\mathrm{C}-\alpha$ cluster of the 160 or any $A 1-\alpha$ bound system produced during the calculation. Thus optimizing the integration step size is a necessity.in this type of calculation.

The following feature is incorporated into the program in order to speed up the computation. Before the computation for the motion of three particles starts, a potential table is created as a function of distance $r$ for each pair of nuclei in the region where the nuclear potential is important. This table is constructed in such a way that the step size $\Delta r$ is small when the nuclear force changes rapidly. In the case of the Woods-Saxon potential, no computation of exponentials are needed in the calculation during the trajectory calculation. At any distance $r_{i k}$ within the range of lable, the furce leru $F_{i k}$ can be computed by the following equation:

$$
F_{i k}(r)=-\left[U_{i k}(n+1)-U_{i k}(n)\right] /\left[r_{i k}(n+1)-r_{i k}(n)\right]
$$

where $i$ and $k$ denote particles $i$ and $k, n$ is the index of the table and $r_{i k}(n+1)$ $\leq r_{i k} \leq r_{i k}(n)$.

Computing the force term with the procedure described above is equivalent to representing the force function with a step-like function. If $\Delta t$ could be so chosen such that the trajectory is always integrated from $r_{i k}(n)$ to $r_{i k}(n+1)$ then energy would be conserved rigorously. However, this requirement cannot be satisfied. When three particles are involved, the exact value of $\Lambda t i k$ for any two particles $i$ and $k$ such that the trajectory is computed from $r_{i k}(n)$ to $r_{i k}(n+1)$ in a three body problem cannot be computed easily. Instead the program estinates $\Delta t_{j k}$ by the following formula:

$$
\Delta \mathrm{r}_{i k}=\mathrm{V}_{i k}{ }^{*} \Delta \mathrm{t}_{\mathrm{ik}}+\left[\mathrm{F}_{\mathrm{ik}} / \mathrm{m}_{\mathrm{ik}}\right] \Delta \mathrm{t}_{\mathrm{ik}}^{2}
$$

At any instant, three different $\Delta t_{i k}$ are derived. Since each of the $\Delta t_{i k}$ is computed approximately, there is no guarantee that the force is computed within one step, $r_{i k}(n)$ to $r_{i k}(n+1)$. Whenever a step is skipped, slight energy non-conservation is introduced. In order to minimize energy non-conservation and at the same time keep the integration step at a reasonable size, $\Delta t$ is always chosen to be the minimum of the three $\Delta t_{i k}{ }^{\prime} s$ such that no more than one step is skipped at any given time $i_{. e} \cdot r_{i k}(n-1)<r_{i k}{ }^{-}<r_{i k}(n)$ or $r_{i k}(n+1)<r_{i k}{ }^{-}<r_{i k}(n+2)$ where $r_{i k}{ }^{-}$is the new position computed from the $\Delta t$ obtained. For the region where the nuclear force is important, $\triangle t$ is of the order of $10^{-24}$ to $10^{-26} \mathrm{sec}$. 
When the force considered is outside the range of the table, only the Coulomb force contributes. In ţhis region, a different criterion is used to determine $\Delta t$ used in the calculation. Since the Coulomb force $F_{c}$ is inversely proportional to $r^{2}, \Delta t$ can be chosen to be proportional to $r$, the relative distance between the two interacting nuclei. Thus, once the nuclei are far apart so that nuclear potential is no longer important, the calculation for the asymptotic trajectories is accomplished very rapidly.

\section{B. Simulation Using Monte Carlo Method}

To completely specify the initial conditions of the. ${ }^{16} 0$ nucleus in any trajectory, it is necessary to know both the impact parameter $b$ for the $16_{0}$ and $27 \mathrm{Al}$ system and the internal coordinates of $\mathrm{C}-\alpha$ bound states. These internal coordinates can be described by the sign of relative velocity and by three spatial parameters; $\mathbf{r}_{\mathrm{c}-\alpha \mathrm{x}}$, the relative distance between carbon and alpha particles, the angular orientation of the $C-\alpha$ with respect to the $1 \mathrm{ab}$, specified by the polar angle and the axial angle.

The Monte Carlo method is used to generate an ensemble of $N$ trajectories. This ensemble samples all allowed initial conditions with corresponding probabilities. Initial values are assigned to each of the input parameters using the random number generator such that the probability distribution for each of these parameters with a large sample size (N) is the same as expected within statistical errors. Random numbers are obtained from the DEC Subroutine RAN which generates uniformly distributed random numbers in the interval $[0,1]$, $0<r<1$. In order to generate the correct ensemble of trajectories, the random numbers generated by RAN are manipulated to create initial trajectory parameters which follow the probability density functions of the parameters.

The new off-line computer VAX/VMS was used in this calculation. Even with this high speed digital computer and modification in computing the force term, it takes one and a half minutes of computer CPU time to process one trajectory. It therefore takes about one day of CPU time to perform 1000 trajectories!

In order to decrease the sample size needed before a three-body break-up event is observed, restrictions obtained through symmetries or through experimental results were placed on the initial parameters that define a unique trajertory. For cxample, frum the fusion and total reaction cross-section, $b_{f u}$ and $b_{\max }$ for $b_{f u} \leq b<b_{\max }$ are estimated to be 6.1 . and $6.7 \mathrm{fm}$. respectively using the sharp cut-off model and unitarity of cross-section. In the calculation, the range of $b$ is relaxed to $4.5 \leq b<7 \mathrm{fm}$. Another example is that only one in-plane $C-\alpha$ relative angle is needed to specify the $C-\alpha$ relative position in the 1.60 nucleus if only the in-plane angular distribution is of importance. 


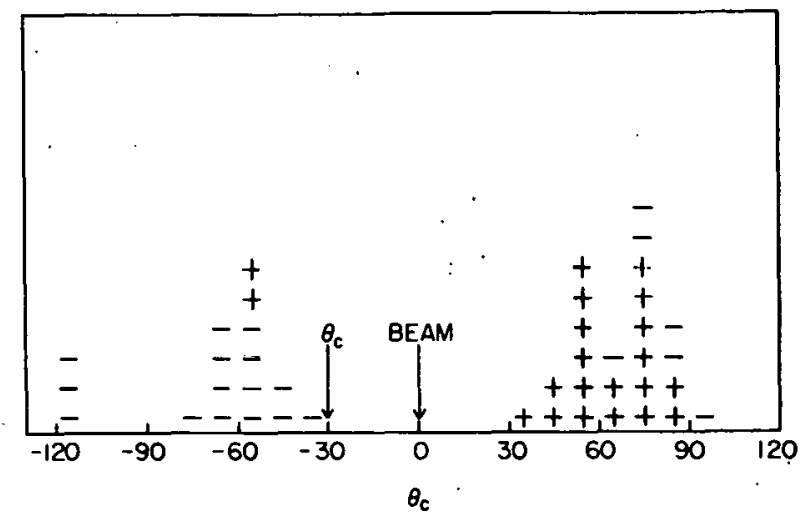

Fig. 10.6.1: C-ar angular distrihution at $\dot{\theta}_{C}==30^{\circ}$ uslut Llie Woods-Saxon potential parameter set I of Tab1e 10.6-1.

C. Results of Calculations

1. Woods-Saxon Potential:

Three different sets of Woods-Saxon parameters listed in Table 10.6-1 were used in the calculation. The results from parameter set $I$ will be discussed first. In this calculation, both in-plane and out-of-plane $C-\alpha$ angles were varied to get all possible $C-\alpha$ orientations in $16_{0}$. 198 out of 5000 trajectories processed yielded three final particles, ${ }^{27} \mathrm{Al},{ }^{12} \mathrm{C}$ and $\alpha$ at the end of the three-body calculation. The remainder of the trajectories are events that yield $A 1-C, C-\alpha$ or $A 1-\alpha$ bound states as final products. of these 198 trajectories, 41 trajectories have the asymptotic outgoing angle of the ${ }^{12} \mathrm{C}$ particle between $25^{\circ}$ to $35^{\circ}$ : Figure 10.6-1 sliuws the in-plane alpha angular correlation in the laboratory system of the events with the ${ }^{12} \mathrm{C}$ angle between $25^{\circ}$ to $35^{\circ}$. This figure can be compared to the pre-equilibrium angular correlation plotted in the lab frame obtained experimentally as shown in Fig. 6.7-5. The angle convention used in Fig. 10.6.1 is the same as that used previously with angles on the same side of the beam as the ${ }^{12} \mathrm{C}$ detector defined to be negative. The angular correlation is broken up into bins of 10 degree width. Each event in the histogram is indicated by a + sign for positive angle (non-orbiting) scattering and a - sign for negative angle (orbiting) scattering of the carbon particle. About twice as many events are scattered to the opposite side of the beam axis from the carbon detector. Most of the trajectories with the alpha partlcles scattered to the same side of the beam as the carbon particles correspond to events where the $C$ particles orbit around the $27 \mathrm{Al}$ nucleus. The angular correlation peaks at $70^{\circ}$ on either side of the beam. It is interesting to see that a "shadow region" similar to the one obtained in the "hot spot" model calculation ${ }^{2}$ is obtained along the beam axis. 
In order to gain insight into the results of the trajectory calculation, a program called 3BODYPLT was written to plot out the projections onto the reaction plane of the positions of the three particles as a function of time. Fig. 10.6-2 is an example of such a plot. In this plot, the positions of the three particles are plotted once about every 200 integration steps. $>, \theta$ and 0 are the symbols for ${ }^{27} \mathrm{Al}, \alpha$ and ${ }^{12} \mathrm{C}$ respectively. The ${ }^{16} \mathrm{O}$ beam moves from left to right in the plot. The $X Y$ plane is the reaction plane containing the beam axis and the carbon detector. The time interval on the figure is the elapsed time between the first and last plotted positions. For this particular trajectory, the $C-\alpha$ bound system oscillates until the alpha partcle feels the nuclear potential of the $A 1$ nucleus. The circle is centered approximately at the initial position of the $A 1$ nucleus. The radius of the circle equals $R_{A 1-\alpha}$. When the alpha particle reaches the edge of the Al-ox nuclear potential, its direction changes and it is scattered to a more backward angle. The alpha particle then moves in the combined potential fields of $A 1-\alpha$ and $C-\alpha$ along a fairly straight line. The alpha particle suffers another more abrupt backward angle scattering when it leaves the nuclear field of Al-o. At this point, the carbon and alpha particles are so far apart that the carbon particle exerts little influence on the alpha trajectory. This is analogous to an object that moves in a. medium and suffers deflection when it crosses from one medium to another one with a different index of refraction. The trajectory shown is a typical one using parameter set $I$.

From the above observation, it becomes clear that the Al- $\alpha$ potential is very important in determining the final results of the three-body trajectory calculation. In order to study this, parameter set II which fits the same ${ }^{27} \mathrm{Al}$ $+\alpha$ elastic scattering data but with a smaller $\mathrm{U}_{\mathrm{A} 1-\alpha}$ was used. In 3000 trajectories, however, no three body final states were observed 1.e. a11 trajectories ended up with two of the nuclei in a bound state. This can be understood since the Al- $\alpha$ potential of set II is not deep enough to break the al pha particle away from the carbon particle in the oxygen nucleus.

Another completely different Woods-Saxon potential (set. III of Table 10.6-1) that fits high energy $\alpha+{ }^{27} \mathrm{Al}$ elastic scattering data was also used. As was the case for set II, no three-body final states were obtained after 3000 trajectories were processed. In this case, the ${ }^{27} \mathrm{~A} 1-\alpha$ potential is so deep that once the alpha particle is attracted by the ${ }^{27} \mathrm{Al}$, the $27_{\mathrm{Al}}$ and $\alpha$ form a bound entity. The results of these calculations are very sensitive to the $A 1-\alpha$ potential. Since no single unambiguious Woods-Saxon potentials can be obtained from the experimental data on elastic scattering of alpha particles on ${ }^{27} \mathrm{Al}$, a different form of nuclear potential, the proximity potential, with minimum adjustable parameters is used. 
Table 10.6-1: Three Woods-Saxon potential parameter sets used in the three-body trajectory calculation

\begin{tabular}{|c|c|c|c|c|c|c|c|c|c|}
\hline & \multicolumn{3}{|c|}{$c-\alpha$} & \multicolumn{3}{|c|}{$A l-C$} & \multicolumn{3}{|c|}{$A 1-\alpha$} \\
\hline & $\boldsymbol{0}(\mathrm{MeV})$ & $a(f m)$ & $R(f m)$ & $\mathrm{U}(\mathrm{MeV})$ & $a(f m)$ & $\mathrm{R}(\mathrm{fm})$ & $\mathrm{U}(\mathrm{MeV})$ & $a(f m)$ & $R(\mathrm{fm})$ \\
\hline I & 24.9 & 0.65 & $4.85 *$ & 35 & 0.55 & $6.08 * *$ & 80 & 0.31 & $7.5^{\mathrm{Q}}$ \\
\hline II & 24.9 & 0.65 & 4.85 & 35 & 0.55 & 6.08 & 34 & 0.31 & $7.5^{\mathrm{a}}$ \\
\hline III & 24.9 & 0.65 & 4.85 & 35 & 0.55 & 6.08 & 218 & 0.68 & $4.72^{\circledR}$ \\
\hline
\end{tabular}

* The values for $a$ and $R$ parameters are obtained from Ref. 3. $U$ is obtained by requiring the calculated root mean square radius for $\mathrm{C}-\alpha$ to be the same as that determined from the experimental charge radii of ${ }^{16} 0,{ }^{12} \mathrm{C}$ and $\alpha$.

** Ref. 4 .

(c) Ref. 5 . 
Fig. 10.6-2: The trajectories of the three, particles $27_{\mathrm{Al}}, \alpha$, and ${ }^{12} \mathrm{C}$ projected onto the reaction plane as a function of time. Parameter set $I$ listed in Table 10.6-1 was used.

Fig. 10.6-3: The trajectories of the three particles ${ }^{27} \mathrm{Al}, \alpha$, and ${ }^{12} \mathrm{C}$. The proximity potentials were used.
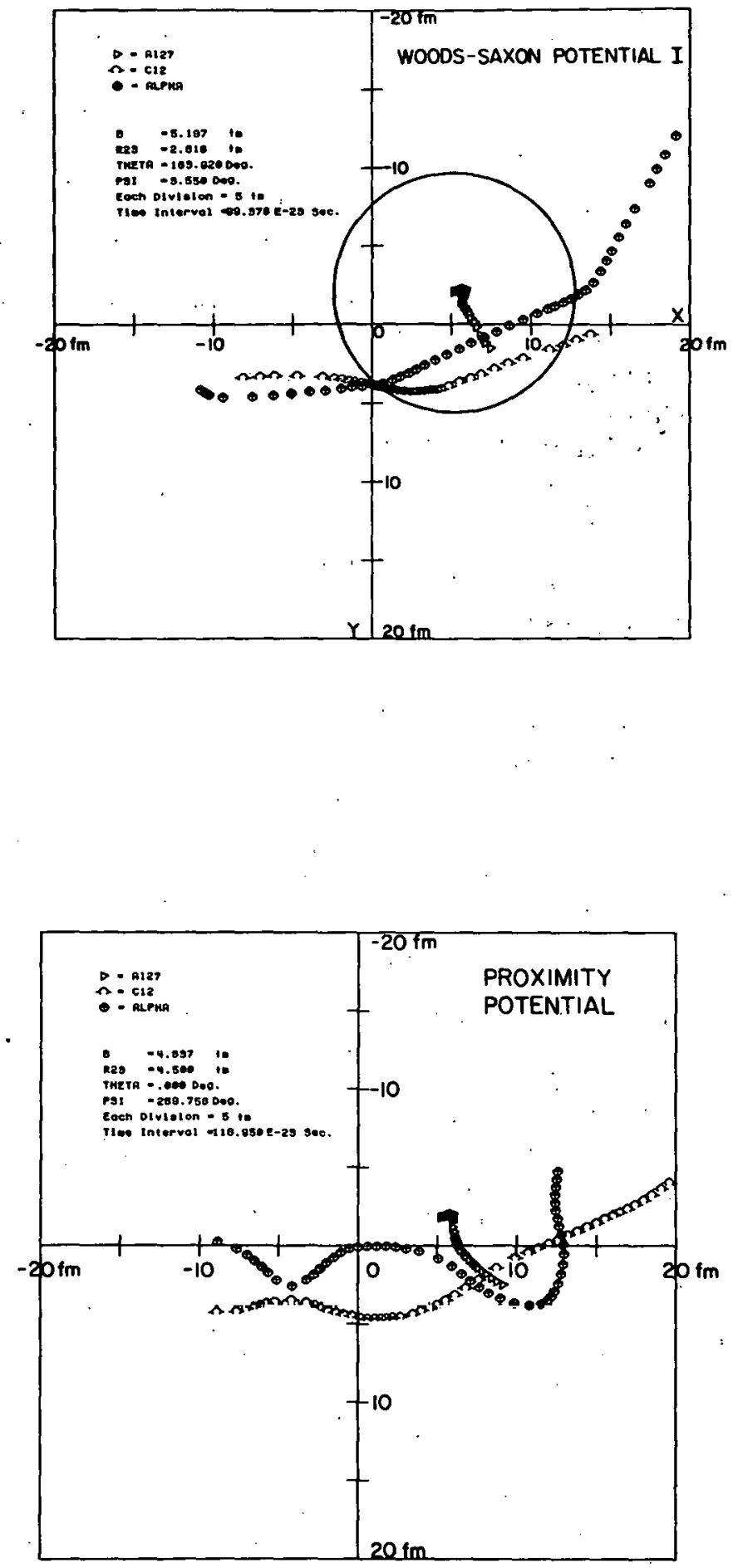
In the case of the proximity potential, in order to optimize the calculation above, the initial positions and velocities of all three particles lie in the reaction plane. Of 3200 trajectories processed, only two trajectories yielded a three-body final state with the asymptotic carbon angle between $25^{\circ}$ to $35^{\circ}$. One trajectory with an asymptotic alpha angle of $90^{\circ}$ is shown in Fig. 10.6-3. The other trajectory yields a more forward alpha scattering angle of $25^{\circ}$. One common feature of trajectories calculated using the proximity potential is that the carbon and alpha particle cannot pass through each other due to the presence of the repulsive core. The internal shape of the proximity potential is responsible in generating the more complicated trajectory as seen in Fig. 10.6-3.

\section{Conclusion}

None of the four different potentials used in the three-body trajectory calculation reproduce the experimental results of the $27_{A 1}\left({ }^{16} U, 12{ }^{10 x}\right){ }^{27} \mathrm{Al}$ system as discussed in Section 6.7. Furthermore, the results of the calculation are very sensitive to the detalled internal structure of the potentials. The slope of the Woods-Saxon Al- $\alpha$ potential causes deflection of the $\alpha$ particles to the more backward angles. It is not clear that other choices of Woods-Saxon potentials would cause the deflection to be in the forward direction. The particle trajectories from the proximity potentials are very complicated and cannot be understood in a simple way. In addition, too few trajectories break up into ${ }^{12} \mathrm{C}, \alpha$ and ${ }^{27} \mathrm{Al}$ with the asymptotic ${ }^{12} \mathrm{C}$ scattered to the desired angle. This prohibits collection of a statistically significant sample to examine the angular distribution of the alpha particles.

The main disadvantage of the calculation described in this chapter is the amount of computer time needed to sample through a large number of trajectories. This greatly restricts the freedom of testing different potential parameters. None of the four potentials used reproduces the experimental angular correlation function as shown in Fig. 6.7-5. This may be attributed to the wrong choice of potentials. It can also be that the assumptions used in the calculation may not be right. For example, the ${ }^{12} \mathrm{C}\left(2^{+}\right)-\alpha$ contribution to the $16_{0}$ configuration may be important or some form of energy dissipation mechanism may need to be incorporated into the calculation to account for core excitation.

\section{References}

1. J. Blocki, J. Randrup, W.J. Swiatecki, and C.F. Tsang, Ann. Phys. 105 (1977) 427 .

2. P.A. Gottschalk and M. Weström, Nucl. Phys. A314, (1979) 232 .

3. R.M. DeVries, Nuc1. Phys. A212 (1973) 207.

4. R. Da Silveira and Ch. Leclerq-Willain, Le Journal de Physique Lettres, 36 (1975) L-117.

5. C.M. Perey and F.G. Perey, Atomic Data and Nuclear Data Table, 17 (1976) 1. 


\section{Calculation of Multiple Scattering Corrections Using a Monte Carlo Method}

\section{W.G. Lynch and M.B. Tsang}

To calculate the multiple scattering corrections to the sub-Coulomb elastic data of Sec. 6.8, it was necessary to write the Monte Carlo code MULSCAT for use on the VAX 11/780 computer. At present MULSCAT calculates the correction to Rutherford scattering due to multiple scattering in the target for the particular target geometry used in the sub-Coulomb measurement of Sec. 6.8. In this geometry, the target foil is perpendicular to the beam and mounted on and downstream of a carbon backing foil. A trivial modification of MULSCAT will permit any arbitrary target angle but this has not been incorporated as yet into MULSCAT. Generalization of MULSCAT to handle other direct reactions besides elastic scattering would be more complicated but still feasible.

At present MULSCAT generates multiple scattering angles using the multiple scattering distributions of $L$. Meyer ${ }^{1}$ which are suitable for low energy heavy particles where $\eta=z_{1} z_{2} / 137 \beta>1$. These distributions are read in as a table and manipulated to obtain the functions needed to generate the multiple scattering distributions randomly using the DEC random number generator RAN. After some investigation, it has become apparent that it will be necessary to match the multiple scattering distributions of Meyer ${ }^{1}$ at larger angles onto the Rutherford cross-section in order' to obtain the necessary accuracy but this has not been done so far.

During the Monte Carlo calculation, MULSCAT generates randomly the multiple scattering angle $\theta_{c}$ caused by the carbon backing foil. A fraction $f$ of the thickness of the target material, e.g., ${ }^{208} \mathrm{~Pb}$ is calculated using RAN. The multiple scattering angle $\theta_{1}$ is generated randomly corresponding to multiple scattering through the fraction $f$ of the target material at an energy loss corrected energy. This angle $\theta_{1}$ is added vectorially to $\theta_{c}$ to obtain $\theta_{i}$. In a similar fashion, the multiple scattering angle $\theta_{f}$ is calculated corresponding to the particle. leaving that target at the measured scattering angle $\theta$ and with an energy loss and kinematically corrected kinetic energy. $\theta_{i}$ and $\theta_{f}$ are used with the measured angle $\theta$ to determine the two-body scattering angle $\theta$. The lab cross-section at $\theta$ is calculated and added to a running sum of cross-sections calculated during previous Monte Carlo trajectories for scattering at $\theta$. This determination of $\theta$ from $\theta_{i}$ and $\theta_{f}$ is not exact since there are random relative azimuthal angles $\phi_{i}$ and $\phi_{f}$. To hasten the convergence of the Monte Carlo simulation, the trajectories are calculated in pairs, one with $\oint_{i}, \oint_{f}$ generated randomly and the next with $\phi_{i}+180^{\circ}, \phi_{f}+180^{\circ}$. This has the effect of rigorously eliminating multiple scattering corrections which are odd order in the multiple scattering angle. These odd order corrections should not be present after an infinite number of Monte Carlo trajectories but would take a great number of trajectories before they would converge to zero. 
MULSCAT generates 3 basic quantities as output. Independent nultiple scattering distributions are printed out for both the target and the carbon backing foil. Also, the running cross-section sums are printed out. These cross-section sums yield the systematic multiple scattering corrections.

\section{References}

1. L. Meyer, Phys. Stat. So1., (6) 44, 253 (1971).

\section{Evaluation of Finite-Geometry Corrections in Depolarization Measurements}

\section{W. G. Weitk.amp}

The polarimeter used in the depolarization measurement described in Sec. 3.5 of this report has a thick helium target and large solid angles to maximize the counting rate. Because cross sections and analyzing powers vary with scattering angle, energy, and the angle between the incident polarization direction and the polarimeter scattering angle, data taken with the polarimeter must be corrected for the effects of finite geometry. A program based on an earlier: program ${ }^{1}$ has been written to do this.

The program establishes a grid of points on each of the apertures which define the range of first scattering angles and the range of second scattering angles and positions. The program then establishes trajectories connecting a grid point in each aperture. Available proton-plus-helium cross section and analyzing power data ${ }^{2}$ are approximated with a polynomial in energy and angle to give values appropriate to each trajectory. The number of particles reaching the detector is calculated for each trajectory and sumed over all trajectories. The program also calculates the detector spectrum. The results of this calculation, which agree well with measurements made with known conditions, are used to provide the effective polarimeter analyzing power as a function of energy required for processing the depolarization data.

\section{References}

1. Nuclear Physics Laboratory Annual Report, University of Washington (19/4), P. 36 .

2. P. Schwandt, T.B. Clegg and W. Haeberli, Nucl. Phys. A163, 432 (1971). 


\section{Software for Analyzing Depolarization Data}

\section{S.K. Lamoreaux and W.G. Weitkamp}

The depolarization measurements described in Sec. 3.5 of this report have produced a large quantity of data and require a relatively complicated analysis. We have developed a system of programs to extract depolarization values from the data.

At each measurement angle,. we take a set of spectra consisting of 4 carbon calibration spectra, 8 copper foreground specträ and 8 background spectra. The copper spectra are taken with incident spin up or down, first scattering angle left or right, and second scattering angle either left or right. The analysis of the data consists of calibrating the energy scales of the spectra, dividing the spectra into bins corresponding to steps in excitation energy in the copper nucleus, combining the binned data into values of the depolarization parameter $D$ in such a way as to minimize instrumental asymetries, and calculating check ratios to check the consistency of the data.

1. Calibration of Spectra Scales Using Carbon Spectra

Before and after taking each set of copper spectra, a carbon target was put in the beam to provide groups of protons entering the polarimeter with known energies. Since the protons pass through a considerable thickness of material in traversing the helium polarimeter; the calibration curves for the polarimeter detectors were not linear, but were approximated with polynomials.

The data were taken with the new data acquisition program SINGLES ${ }^{1}$, and written on tape. The primary program used in this part of the analysis was DEPOL. Subroutines include MAGTA, which read blocks of data from tapes written by SINGLES; READRUN, which made a list of run titles and numbers of a tape volume; TEST, which provided a visual check of the data read; and PLOT, which plotted the carbon data in a form which made it easy to establish peak channels by eye. The program CALIB was used to calculate the energy of the protons leaving the relatively thick carbon target. Peak channel numbers and the output of CALIB were inputs to DEPOL subroutine ENERGY, which calculated coefficients for the calibration polynominals mentioned above.

\section{Extraction of Binned Data from Copper Spectra}

With calibrated detectors, it was possible to divide the copper spectra into bins corresponding to excitation energy in copper. A total of 14 bins were used, $1 \mathrm{MeV}$ wide at low excitation energies and $0.5 \mathrm{MeV}$ wide at high energies. Program CALIB calculated proton energies incident on the polarimeter corresponding the excitation energy steps. DEPOL read appropriate spectra from the SINGLES tape as in the calibration part of the analysis described above. DEPOL subroutine ADD used the ouput of CALIB and ENERGY as input and summed the counts in the bins. The output consisted of 14 numbers for each spectrum, which were printed for visual checking. 


\section{Combination of Binned Data into Values of $D$}

In this section of the analysis, we used a modification of the procedure described in Ref. 2. The number of counts in the bin centered on given excitation energy at a given angle with incident beam polarization in the positive direction ( $(t)$, first scattering angle left (1), and second scattering angle right $(R)$, is denoted $N(+, l, R)$. In an double scattering experiment of the type considered here, $N$ is given by

$$
N=I( \pm) \quad \ell(L, R)\left[1+\alpha \beta p_{b} A_{y}+\beta \gamma\left(p^{y^{-}}+\alpha \beta p_{b} D\right) A_{p}\right]
$$

where $I$ is a factor proportional to the number of protons passing through the target when the beam polarization is in a given direction, 0 is the detector solid angle, $\mathrm{P}_{\mathrm{b}}$ is the beam polarization; $\mathrm{A}_{\mathrm{y}}$ is the analyzing power in the first scattering, $\mathrm{PY}^{\mathrm{y}}$ is the polarization resulting from an unpolarized incident beam, $D$ is the depolarization, and $A_{p}$ is the analyzing powar of the helium polarimeter. The factors $\alpha, \beta$, and $\gamma$ are equal to $+1(-1)$ if the incident beam polarization is positive (negative), first scattering left (right), and second scattering left (right), respectively.

The eight equations represented by eq. 1 can be reduced to four by combining those pairs of equations which have the same signs on all terms on the right side. We define four numbers, $L^{+}, L^{-}, R^{+}$, and $R^{-}$for each energy bin, where, for example:

$$
\mathrm{L}^{+}=[\mathrm{N}(+, \ell, \mathrm{L}) \cdot \mathrm{N}(-, \mathrm{r}, \mathrm{R})]^{1 / 2}
$$

These are formed into two combinations:

$$
\text { et }=\frac{L^{+}-R^{+}}{L^{+}+R^{+}} \pm \frac{L^{-}-R^{-}}{L^{-}+R^{-}}
$$

In these combinations, we assume that the ratios of $Q(L)$ to $\varrho(R)$ and $I(+)$ to $I(-)$ remain constant when the first scattering angle is changed so that the $I$ and 2 factors cancel out. We also assume that that the beam polarization magnitude is independent of polarization direction. This last condition is not completely satisfied since the beam polarization does change by as much as $10 \%$ when the direction is reversed. However, the averaging in the rest of the calculation reduces this error to a value well below other uncertainties. Eqs. 1 and 2 can be solved for D:

$$
D=\left[e^{-/ p_{b}}+e^{+} A_{y}\right] / 2 A_{p}
$$

In order to evaluate this equation we must have a value for $A_{y}$, the analyzing power. Our data set contains all the information we need to do this. For each bin, we form the combinations $M_{l}$ and $M_{r}$, where

$$
M_{\ell}=\frac{N(+, \ell, L)+N(+, \ell, R)}{N(-, \ell, L)+N(-, \ell, R)}
$$


Using $r=\left(M_{\ell} / M_{r}\right)^{1 / 2}$; we can solve for $A_{y}$ :

$$
A_{y}=\frac{r-1}{P_{b}(r+1)}
$$

The computer program FINAL, using as input the output from DEPOL, calibration data for the helium polarimeter, and results of separate calculations of the average beam polarization, combined foreground and background binned data, and evaluated eq. 3 to produce values of $D$. FINAL also calculated both systematic and statistical uncertainties in $D$, which were used in appropriate ways to combine $D$ values measured in different runs, and which were quoted in the final results.

4. Calculation of Check Ratios

The program FINAL calculated foun values of the form:

$$
Q_{1}=\frac{N(+, l, L) \cdot N(-, l, L)}{N(-, r, R) \cdot N(+, r, R)} 1 / 2
$$

which give ratios of the factors $\underline{Q}$ and $I$ in eq. 1 above. In this case, for example, $Q_{1}=\ell(L) / \Omega(R)$. These check ratios provide an independent check on the quality of the data, since any value which deviates from the average value. near unity signals problems with the data collection system. Data points which showed such deviations were discarded from the analysis.

\section{References}

1. Nuclear Physics Laboratory Annual Report, University of Washington (1979), p. 162 .

2. R.A. Hardekopf and D.D. Armstrong, Phys Rev. C13, 900 (1976).

10. A Text-Processing System for the Preparation of Scientific Dósuments with the VAX Computer System and the QUME Terminal

\section{J.G. Cramer}

The VAX 11/780 computer system, which has been in operation for about one year, provides the basis for a relatively powerful word processing system for the preparation of multiple-copy correspondence, scientific papers, theses, and other documents when used with a high-quality terminal/typewriter such as the QUME $^{1}$ word-processing terminal which was acquired by the Laboratory last year. However, a serious limitation in the preparation of such documents is the need for superscripts and exponents, for subscripts, and for special characters which are an important part of most scientific documents. 
In principle, this problem can be dealt with by changing the "daisy-wheei" of the QUME terminal to one which contains Greek letters and mathematical symbols and is available from the manufacturer. However, this technique proved unsatisfactory because of the time and care required in changing the "daisy-wheel" and the difficulty in positioning the paper so that the special characters were properly located during the pass in which they were typed over the. normal type script.

Therefore, we have devised a simple and relatively trouble-free solution to this problem which has been in successful operation for the past six months. The QUME terminal has a plot mode which, after invoking a certain escape sequencc, permits the vertical positioning of the typed character to an accuracy of about 1/48" and a horizontal accuracy of about 1/120". "lhis feature has been cmployed to position che carllage beforc and aftor the typing nf superscripts and subscripts, and for the production of special symbols by carefully positioning and striking over the standard ASCII character set of the QUME. Table 10.19-1 shows a photographic reproduction of the special characters and symbols generated by the QUME when operated in this mode.

The protocol for specifying these characters, was devised so as to be "transparent" to the DEC text preparation program Runoff (RNO). This was accomplished by employing ASCII control characters to indicate "special handling" for the character which they follow. In particular, the control characters ${ }^{\wedge} P,{ }^{\wedge} D,{ }^{\wedge} E,{ }^{\wedge} F$, and ${ }^{\wedge} T$ (which have no particular significance for RNO or the text-editing utilities) are used as flags indicating, when following a particular character, that the character is a special character ( $\left.{ }^{\wedge} P\right)$, that it should have a vector arrow over it $\left({ }^{\wedge} D\right)$, or that it should have a t1lde over it $\left({ }^{\wedge} E\right)$, or a bar over it $\left({ }^{\wedge} F\right)$, or a caret over it ( $\left.{ }^{\wedge} T\right)$. In a similar way, the control characters ${ }^{A} A$ and ${ }^{A} B$ are used to indicate that the following text is to be vertically positioned a half-line above ( $A$ ) or below ( ${ }^{\wedge} B$ the preceeding text in the line. Thus $x$-squared is written $x^{\wedge} A 2^{\wedge} B$, while $x-s u b 8 c r i p t-2$ is written $\mathrm{x}^{\wedge} \mathrm{B} 2 \wedge \mathrm{A}$, with this protocol. The principal reason for employing control characters as flags in this way is that RNO docs not include control characters in its character count. used in right-justifying and filling lines of text, and so the presence of control characters in the text line does not interfere with the text justification process.

The implementation of the actual. QUME commands for constructing the characters and positioning the carriage has been accomplished by mcans of a set of procedure files stored in the library area of the VAX disc and used in conjunction with the very powerful DEC editor utility TECO. A command file called QUME.CMD is invuked (by typing (QUME), asks a few questions pertaining to the file name, the degree of vertical offset for superscripts and subscripts, and whether the entire file or only selected pages are to be. typed, and then submits the procedure to the VAX batch queue for typing on the QUME when that device becomes available. The user sees very little of the actual procedures used in this process and is not rcquired tn learn very much protocol to use the system. A "help" file is available in the VAX HELP system to provide information describing the protocol for text preparation. 
Table 10.10-1: Table of Qume Special Symbols

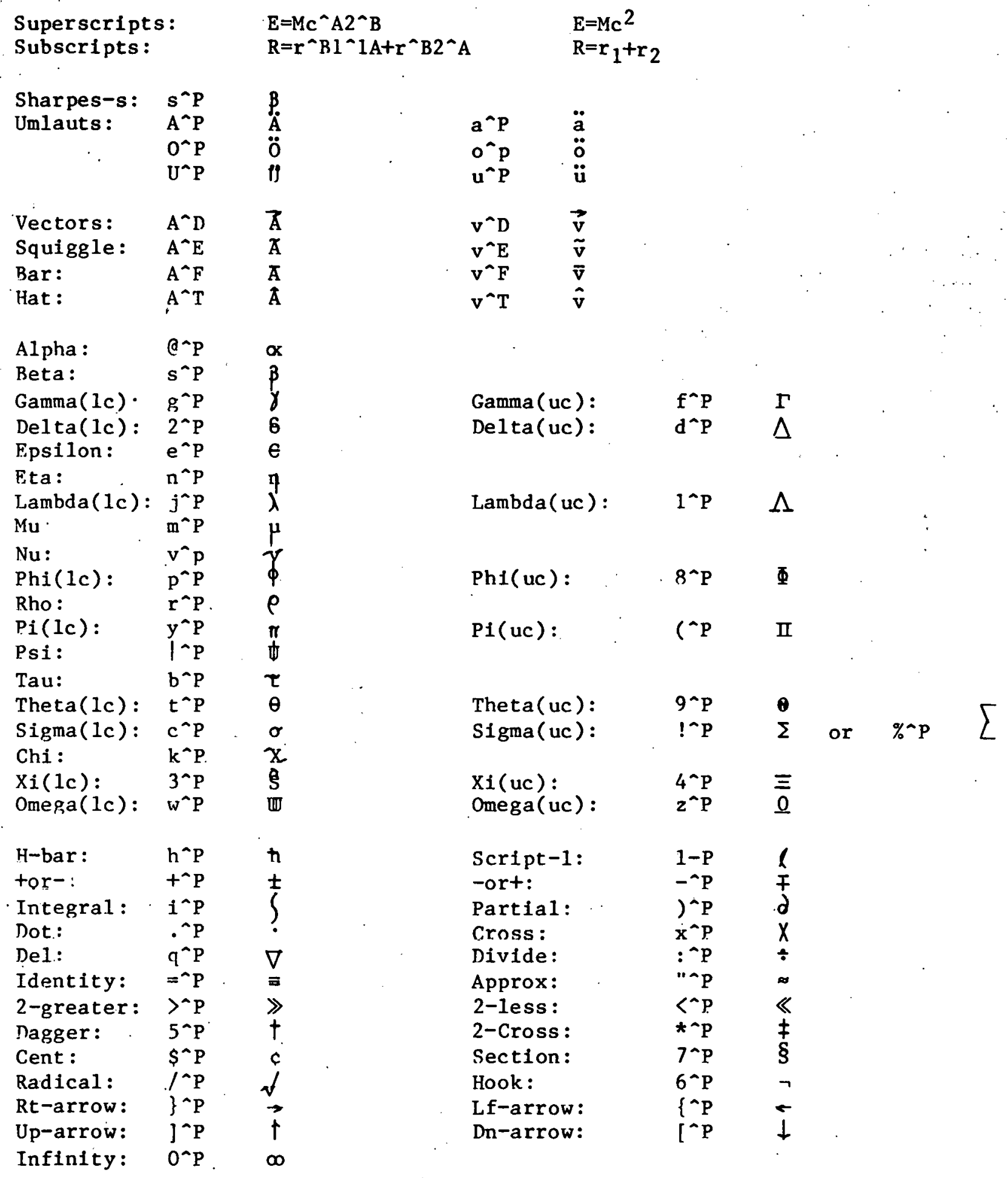


Experience with this system indicates that the protocol described above is a simple and easily used extension of the normal RNo protocol for the preparation of scientific documents, and has in some cases permitted the preparation of a manuscript for submission to a journal "untouched by human hands", i.e., which does not require further insertion of symbols, etc., before mailing. Several $\mathrm{PhD}$ theses have been prepared with this system, and several advantages of the procedure have become apparent. It is very fast and easy to correct, insert, and rearrange text including equations and special symbols. A large fraction of the equations in a typical thesis can be produced without further typing. Students have, in most cases, been able to type their own theses, using the same text-editor system which they employed in preparing programs for the VAX. Margins and other requirements imposed by the Graduate School can be rigorously observed. Further, since the text is prepared in this computer-readable format, it is available for other computer-based processing. One example of this is the possibility of submitting the computer-prepared text directly to an ATP journal ovcr telephoile lines. Another example of the power of this arrangement is SPELL, a text verificatinn syotem rocently inslalled on the VAX tor rhesking the spelliug of words in such text by direct comparison with a "dictionary". which is resident on the VAX disc. Many of the articles in this present Annual Report were prepared directly by the authors employing the text-preparation system described above, and have been "proof-read" by the VAX, using the SPELL text verification system.

\section{A Computer Program to Analyze Experiment 191 Data from LAMPF}

Konrad Aniol

Since we acquired a VAX $11 / 780$ computer in June of 1979 we have the facility for sorting and analyzing our $\left(\pi, \pi^{-}\right)$data here at the Nuclear Physics Laboratory. Previously the data tapes were analyzed either in Los Alamos or at Carnegie-Mellon University in Pittsburgh, where a sorting routine (called DEUS running under DEC-DOS was employed.

The data to be sorted consists of 16 linear signals from the CMU 8 crystal germanium detector, 12 logic signals from 3 stacks of XY wire chambers and several logic signals generated by plastic scintillators for strobe triggering or veto purposes. In all we collect 36 parameters per event.

In the sorting routine written here at the Nuclear Physics Laboratory we employ a range-energy table look up procedure for the particle identification. The procedure used in DEUS is the standard one employing the approximate range energy relation

$$
R(E)=\sigma E \beta
$$

For the table look up technique we need only store the proton range-energy table. The range-energy relation for other particles can be easily obtained from the proton table. Another difference between our sorting routine and DEUS is the use of $X^{2}$ cut on the PID. Once having obtained the PID, the PID subroutine calculates the expected energy deposition in each of the crystals in 
the stack. A $X^{2}$ sum of the measured and calculated energy differences is made in the usual way. Explicitly,

$$
X^{2}=\sum_{i=1}^{\text {ISTP-1 }} \frac{E_{i}^{\text {meas }}-E_{i}^{c a l s^{2}}}{\left(0.10 * E_{i}\right)} /(\text { ISTP-1) }
$$

Here $E_{i}$ is the total energy lost up to detector $i$. The factor 0.10 multiply $E_{i}$ in the weighting factor is determined experimentally to give the observed full wid.th-half maximum straggling for a monochromatic $\pi^{+}$beam on the 8 stack detector. The advantage in using the $X^{2}$ cut lies in the full use of all the information we have about a particular event. Preliminary sorting of the tapes indicates that the $X^{2}$ cut diminishes some of the structure we observe in the $\left(\pi, \pi^{-}\right)$spectra.

It is difficult to compare DEUS with the new sorting routine. DEUS runs in a single user DOS environment, and is a rather more general code. DEUS typically sorts a tape in 100 minutes whereas the program we use here can' sort a tape in about 25 minutes, even under a light to moderate usage multi-user environment. Most likely, the increase in sorting speed is attributable to the performance of the VAX over the older DOS.

12. ADJJ--A Program to Calculate Angular Distribution Coefficients for (particle, $\gamma$ ) Reactions

P.G. Ikossi

The angular distributions of the $\gamma$-rays in a capture reaction is written as

$$
\sigma(E, \theta)=\sum_{K=0}^{2 L} A_{K}(E) Q_{K} P_{K}(\cos \theta)
$$

$$
\sigma(E, \theta) \mathrm{A}_{\mathrm{y}}(E, \theta)=\sum_{\mathrm{K}=1}^{2 \mathrm{~L}_{\max }} \mathrm{B}_{\mathrm{K}}(E) \mathrm{Q}_{\mathrm{K}} \mathrm{P}_{\mathrm{K}}{ }^{1}(\operatorname{cs} \theta)
$$

where $A_{K}$ and $B_{K}$ can be written

$$
A_{K}=\sum_{t t^{-}} D\left(t t^{-}, k\right) \operatorname{Re}\left(S t S t^{\prime}\right)
$$

and 


$$
B_{K}=\sum_{t t^{-}} f_{K}\left(t t^{\prime}\right) D_{t t^{-}} \cdot \operatorname{Im}\left(S_{t} S^{*} t^{-}\right)
$$

Here St, $\mathrm{St}^{-}$are the reaction matrix elements for the channels + and $+^{-}$, and

$$
f_{K}\left(t t^{-}\right)=\left[J^{-}\left(J^{-}+1\right)+\lambda(\lambda+1)-J(J+1)-\lambda^{-}\left(\lambda^{-}+1\right)\right] / K(K+1)
$$

The angular momentum coupling factors $D\left(t t^{-}, k\right)$ have been tabulated for target and residual spins $\leq 5 / 2$ by Carr and Baglen. 1 When higher spins are involved the evaluation of these factors from tabulated values of vector coupling coefficients becomes tedious. Nevertheless they are necessary for the interpretation of $\gamma$-ray angular distributions.

The program ADJJ overcomes this difficulty. For given projectile, target and final-state spins and parities and multipolarities of the $\gamma$-rays it tabulates the coefficients for this expansion in the J-J coupling scheme for a11 possible J-values of the initial state. The program.uses the appropriate formula given by Sharp et al. ${ }^{2}$ modified to agree with the phase convention of Ref. 1.

\section{$\underline{\text { References }}$}

1. R.W. Carr and J.E.E. Baglin, Nuclear Data Tables 10, 143 (1971).

2. W.T. Sharp, J.M. Kennedy, B.J. Sears, and M.G. Hoyle, AECL-97 (1967). 
11. APPENDIX

1. Nuclear Physics Laboratory Personnel.

Faculty

Eric G. Adelberger, Professor

John S. Blair, Professor

navid Bodansky, Professor, Chairman, Department of Physics

John G. Cramer, Professor

George W. Farwe11, Professor

I. Hal pern, Professor

Fred H. Schmidt, Professor

Kurt A. Snover, Research Associate Professor

Thomas A. Trainor, Research Associate Professor

Robert Vandenbosch, Professor; Director, Nuclear Physics Laboratory

Willian G. Weitkamp, Research Professor; Technical Director Nuclear Physics Laboratory

Research Staff

Konrad Aniol, Research Associate

Hubert Doubre, Research Associatel

Pitsa Ikossi, Research Associate

Albert J. Lazzarini, Research Associate

Volker Metag, Research Associate

Eric B. Norman, Research Associate

Raymond J. Puigh III, Research Associate 2

Ruedi Risler, Research Associate

Senior Professional Staff

Harold Fauska, Reseach Electronics Supervisor, Assistant Tcchnical Director, Nuclear Physics Laboratory

Derek Storm, Senior Research Scientist

Predoctoral Research Associates

Norman L. Back

Hyoung C.: Bhang

Yuen-dat Chan 3

David T.C. Chiang 4

Keith J. Davis

Timothy E. Chupp

David W. Holmgren

C. David Hoyle 
Zafar Iqbal

Kevin T. Lesko

Robert Loveman

Will1an G. Lynch

Man-Yee B. Tsang

Richard D. Von Lintig

Research Assistants

Salvador Gi1

Dat-Kwong Lock

Professional Staff

John F. Amsbaugh, Research Scientist

Michael Bizak, Research Scientist 5

Kelly C. Green, Research Scientist

Ge ivas M. Hinn, B.esparr.h Scientist

William B. Ingalls, Research Fngineer

Donald D. Leach, Research Scientist

Alän $G$. Sealuster, Rcocaroh lingineer

Richard J. Seymour, Computer Systems Engineer

Rod E. Stowell, Electronics Engineer

H. Erik Swanson, Research Scientist

Technical Staff

Carl E. Linder, Engineering Technician

Georgia J. Rohrbaugh, Accelerator Technician

George E. Saling, Accelerator Technician

Louis L. Geissel, Instrument Maker Leadman, Student Shop

Gustav E. Johnson, Instrument Maker

Hendrik Simons, Instrument Maker Leadman

Peter $W$. Wiest, Accelerat or Operator 6

Allen L. Willman, Iusliument Maker Supervisnr.

Administrative Staff

Julie L. Anderson, Accounting Assistant

Dianne S. Hulford, Administrative Secretary

Part Time Staff

Gregory L. Andersen 7

Tim Bertram

Ambrose $\mathrm{Cl}_{\text {latil }} 7$

Richard Demonaz 7

Alberto Ferriera

Eric Geissel

Daniel Giles?

Dale Ilirt

James $W$. Jones 7

Kwi Y. Kim7

Mark Klebanoff ${ }^{7}$

Steven Lamoreaux

Jim Martynovyrah 7

Leslie Pence

David Peterson

Duncan Prindle

Michael Rust

Winston A. Saunders

Theo Schaad ${ }^{7}$

Tim Van Wechel

Christopher Wagner

Mark E. Warchol 
1. Present address Jaboratoire de Physique Nucleaire, Orsay, France.

2. Present address: Westinghouse, Hanford, WA.

3. Completed Ph.D. degree. Present address: Oak Ridge National Jaboratory, TN.

4. Completed Ph.D. degree. Present address: The Boeing Company, Seattle, WA.

5. Present address: University of California, Riverside, CA.

6. Present address: Department of Oncology, University of Washington.

7. No longer associated with the Nuclear Physics Laboratory

2. Ph.D. Degrees Granted, Academic Year 1979-80

Yuen-dat Chan: Gross Structures in Reactions Between the Carbon and Oxygen Isotopes and a Comparative Study of the Elastic $180^{\circ}$-Excitation Functions for $\mathrm{p}-$ and sd-shell HeavyIons at Low Bombarding Energies

David T.C. Chiang: Low Energy Inelastic Pion Scattering to the Continuum

3. List of Publications

Papers Published:

"Improved Test of Nucleon Charge Conservation," E.B. Norman and A.G. Seanster, Phys. Rev. Lett. 43, 1226 (1979).

"The $\alpha-$ Nidth of the Lowest $\mathrm{T}=2$ state in ${ }^{28} \mathrm{Si}, "$ P.G. Ikossi, K.A. Snover, J.L. Osborne, E.G. Adelberger, and A.B. McDonald, Nuclear Physics A319, 109 (1979).

"Observation of Magnetic Dipole Strength in $16_{0}, "$ K.A. Snover, P.G. Ikossi, and T.A. Trainor, Phyc. Rev. Lctt. 43, 117 (1979).

"Relative Y1elds of $26_{\mathrm{Al}} g$ and $26_{\mathrm{Al}}^{\mathrm{m}}$.from $25_{\mathrm{Mg}}(\rho, \gamma)$ Reaction," E. B. Norman, Astrophys. J. 231, 198 (1979).

"Decays of the Lowest $\mathrm{T}=2$ State in $\mathrm{N}=4 \mathrm{~N}$ Nuclei from ${ }^{8} \mathrm{Be}$ to ${ }^{44} \mathrm{Ti}$," S.J. Freeman, C.A. Gagliardi, M.A. Oothoudt, A.V. Nero, R.G.H. Robertson, F.J. Zutavern, E.G. Adelberger, and A.B. McDonald, Phys. Rev. C19, 1907 (1979).

"Half-Life of $176 \mathrm{Lu}, "$ E.B. Norman, Phys. Rev. C21, 1109 (1980).

"Unique Determination of the Amplitude and Phase for the Population of the Giant-Dipole Resonance in the Reaction ${ }^{2}{ }^{C}\left(p_{p o l}, \gamma_{0}\right){ }^{13}$ N, " K.A. Snover, P.G. Ikossi, E.G. Adelberger, and K.T. Lesko, Phys. Rev. Lett. 44, 927 (1980). 
"Forward Amplitudes for $\pi^{ \pm}$on $\mathrm{Al} \mathrm{Ca} \mathrm{Cu} \mathrm{Sn}$ Ho and $\mathrm{Pb}$ in the Energy Range 65-215 MeV," R.H. Jeppesen et al. (Collaboration of Experiment 2, LAMPF.) Proceedings Second Internat. Topical Conference on Meson-Nuclear Physics Houston (1979). E.V. Hungerford Editor.

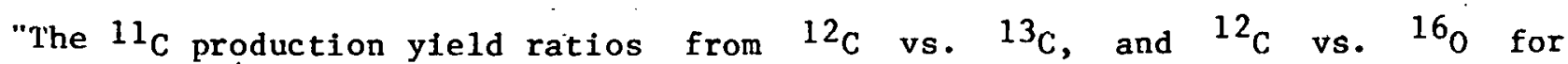
incident $\pi^{ \pm}$from 100 to $250 \mathrm{MeV}, " \mathrm{~K}$. Aniol et al. (Collaboration of experiment 324.) Proceedings Second Internat. Topical Conference on Meson-Nuclear Physics. E.V. Hungerford, Editor, p. 276.

"Simple Microscopic Model for Nuclear Alignment in Peripheral Nuclear Collisions," R. Vandenbosch, Phys. Rev. C20, 171 (1979).

" $Q$ and $Z$ Dependence of Angular Momentum Transfer in Deeply Inelastic Collisions of $86_{\mathrm{Kr}}$ with $209_{\mathrm{B1}}, "$ P. Dyer, R.J. Pulgh, R. Vandenbosch, T.D. Thomas, M.S. Zisman, and L. Nunnelley, Nuclear Physics, A322, 205 $(1979)$.

"Magnitude and Alignment of Transferred Angular Momentum in Both Quasi and Deeply Inelastic Scattering," R.J. Puigh, P. Dyer, R. Vandenbosch, T.D. Thomas, L. Nunnelley, and M.S. Zisman, Phys. Lett., Vol. 86B, No. 1.

"On the Origin of Oscillations in the Fusion Cross Section of ${ }^{12} \mathrm{C}+12 \mathrm{C}$," R. Vandenbosch, Physics Letters, 87B, 1979.

"Alignment of Transferred Angular Momentum in Deaply Inelastic Collisions from Discrete $\gamma$-ray Angular Correlations," R.J. Puigh, H. Doubre, A. Lazzarini, A. Seanster, R. Vandenbosch, M.S. Zisman, and T.D. Thomas, Nucl. Phys. A336, 279 (1980).

"On the Variations in Fusion Cross sections for Different Light Heavy Ion Systems," R. Vandenbosch, Nucl. Phys. A339, 167 (1980).

Papers Submitted or in Press:

"Radiometric Dating with the University of Washington Tandem Van de Graaff Accelerator," G.W. Farwell, T.P. Schaad, F.H Schmidt, M-Y.B. Tsang, P.M. Grootes, and M. Stuiver, to be published in Radiocarbon.

"Enrichment of ${ }^{14} \mathrm{C}$ and Sample Preparation for $\beta$-Decay and Ion Counting," P.M. Grootes, M. Stuiver, G.W. Farwell, T.P. Schaad, and F.H. Schmidt, to be published in Radiocarbon.

"Magnetic Moment of the $3^{+}$, One Millisecond Isomer in Self-Conjugate ${ }^{46} \mathrm{~V}$," R. Sielemann, D. Burch,' B. Cuengeo, K. Aniol, Y.-D. Chan, H. Fauska, and W.G. Lynch, submitted to Phys. Lett. B.

"Generalized Absorber Theory and the Einstein-Podolsky-Rosen Paradox," J.G. Cramer, to be published in Phys. Rev. D. 
"Dominance of Strong Absorption in ${ }^{9} \mathrm{Be}+{ }^{28} \mathrm{Sr}$ Elastic Scattering," M.S. Zisman, J.G. Cramer, D.A. Goldberg, J.W. Watson, and R.M. DeVries, to be published in Phys. Rev. C.

"The Radial Sensitivity of Elastic Scattering," J.G. Cramer and R.M. DeVries, to be published in Phys. Rev. C. .

"Charge Distributions for the ${ }^{86} \mathrm{Kr}+139 \mathrm{La}$ system at 505,610 , and 710 MeV," P. Dyer, M.P. Webb, R.J. Puigh, R. Vandenbosch, T.D. Thomas, and M.S. Zisman, submitted to Phys. Rev. C.

"Cross Sections Relevant to Gamma-Ray Astronomy: Proton Induced Reactions," P. Dyer, D. Bodansky, A.G. Seamster, E.B. Norman, and D.R. Maxson, submitted to Phys. Rev. C.

"A Technique for Measuring Parity Nonconservation in Hydrogenic Atoms," E.G. Adelberger, T.A. Trainor, E.,N. Fortson, T.E. Chupp, D. Holmgren, M.Z. Iqbal, and H.E. Swanson, to be published in Nucl. Inst. Meth.

Invited Papers and Talks:

"Parity Mixing in Hydrogen at Seattle," T.A. Trainor, Workshop on Neutral Current Interactions in Atoms, Cargese, Corsica, Sept. 1979.

"Giant Resonance Effects in Radiative Capture," K.A. Snover, The Oak Ridge Giant Multipole Resonance Topical Conference, Oak Ridge, October 1979 (to be published).

"Energy Dissipation and Angular Momentum Transfer in Collisions Between Heavy Nuclei," R. Vandenbosch, German Physical Society, Munich, March 1980.

"Experimental Search for a Fusion Window," R. Vandenboṣch, International Symposium on Heavy Ion Fusion Reactions, Bad Honnef, Germany, March 1980.

\section{Contributed Abstracts:}

"Detection of Linear Polarization of X-Rays and Gamma-Rays Using Pulse Rise Time," R.A. Loveman and J.G. Cramer, BAPS 24, 823. (1979).

"Proton Depolarization In Inelastic Scattering From Copper," W.G. Weitkamp, T.A. Trainor, H. Bhang and S.K. Lamoreaux, BAPS 24, 838 (1979).

"An Improved Test of Nucleon Charge Conservation," E.B. Norman and A.G. Seamster, BAPS 24,827 (1979).

"A Unique Amplitude and Phase Determination for the ${ }^{12} \mathrm{c}\left(\mathrm{p}, \gamma_{\mathrm{o}}\right)$ Reaction," K.A. Snover, P.G. Ikossi, E.G. Adelberger, and K.T. Lesko, BAPS $\underline{24}$, 844 (1979).

"The Role of the 6.3-s ${ }^{26} \mathrm{Al}$ 畐 in the Nucleosynthesis of ${ }^{26} \mathrm{Al}, "$ E. B. Norman, BAPS 24, 63 (1979). 
"Analyzing Powers in the Continuum Portions of Particle Spectra for 18 $\mathrm{MeV}$ Proton Bombardment of ${ }^{63} \mathrm{Cu},{ }^{64} \mathrm{Zn}$ and ${ }^{103} \mathrm{Rh}, "$ H.C. Bhang, N. Back, J.S. Blair, I. Halpern, W. Lynch, G.A. Miller, and T.A. Trainor, BAPS 24, 829 (1979).

"A Search for Non-Fusion in the Reaction $16_{0}+16_{0}$ at $\mathrm{E}_{\mathrm{cm}}=34 \mathrm{MeV}$," A.J. Lazzarini, H. Doubre, A. Seamster, and R. Vandenbosch, BAPS $\underline{24}$, 852 (1979).

"Search for Instantaneoous Fission," V. Metag, H. Doubre, A. Lazzarini, E. Norman, R. Puigh, A. Seamster, R. Vandenbosch, and M. Zisman, BAPS 24, 826 (1979).

"Relativistic Coulomb Effects in Heavy Ion Elastic Scattering," W.G. Lynoh and .I.li. Uramer, BATS 24,843 (1979).

"Coincidence study of the ${ }^{27} \mathrm{Al}\left({ }^{16} \mathrm{O},{ }^{12} \mathrm{C} \alpha\right)^{27} \mathrm{Al}$ Reaction at $65 \mathrm{MeV}, " \mathrm{M} . \mathrm{B}$. Tsang, W.G. Lynch; R.J. Puigh, A. Seamster, and R. Vandenbosch, BAPS 24, 833 (1979).

"UW Parity Violation in Hydrogen: A Progress Report," T.A. Trainor, E.G. Adelberger, T. Chupp, K. Davis, D. Hoyle, M: Iqbal., W.B. Ingalls, and H.E. Swanson, BAPS 24, 618 (1979).

"Ji Assignments in $29_{\mathrm{P}}$ From Polarized Proton Scattering on ${ }^{28_{S i}}$, P.G. Ikossi, K.A. Snover, E.G. Adelberger, and Y. Haque, BAPS 24, 613 (1979).

"An Effective Reflection-Type Geomerry for Sputter Ion Sources," F.H. Schmidt and G.W. Farwell, BAPS 24, 650 (1979). 


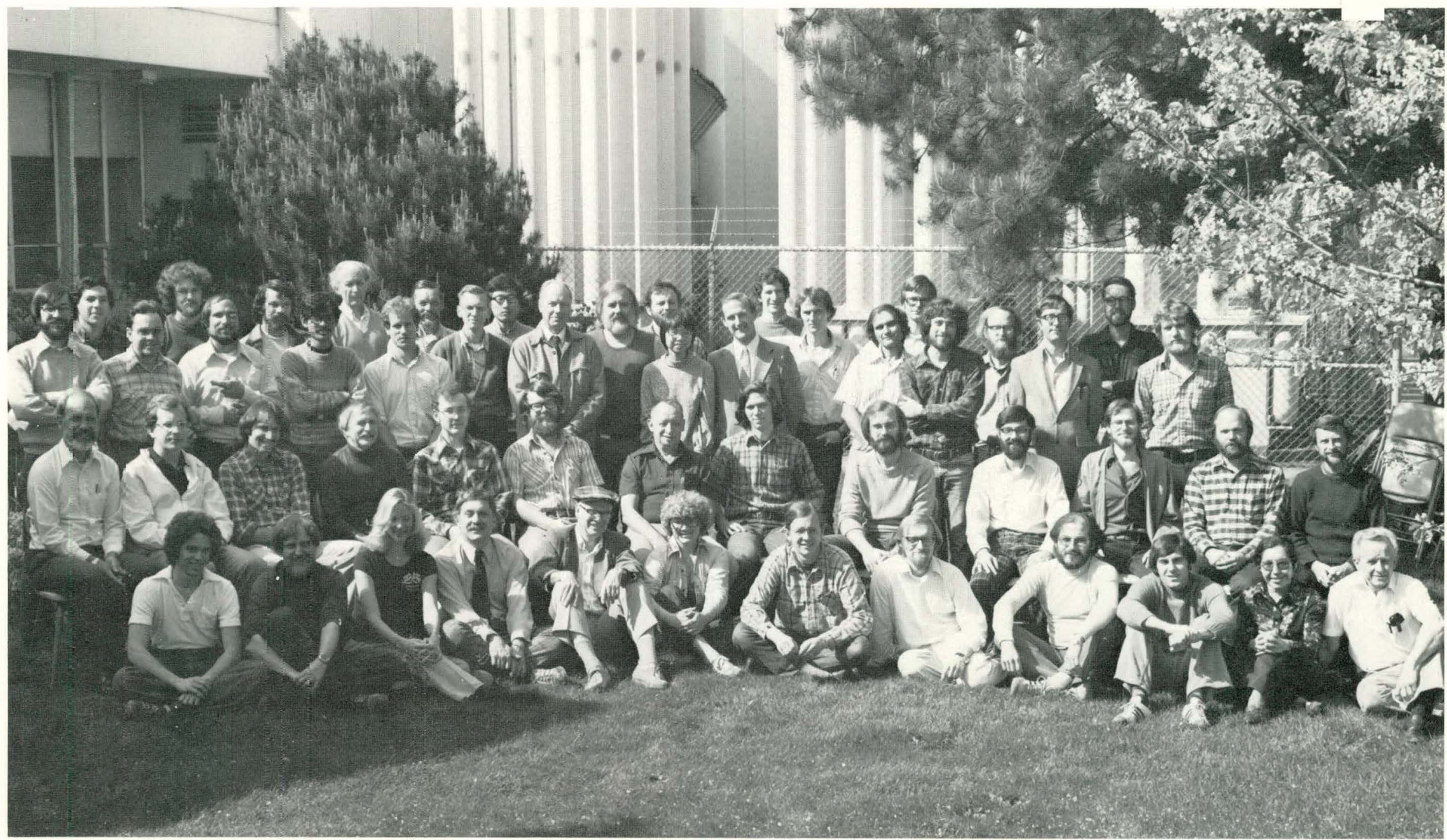

TOP ROW: Norman, Amsbaugh, Ingalls, Halpern, Willman, Bhang, Hoyle, Lynch, Metag, Back

2ND DoWN ROW: Trainor, Risler, Adelberger, Iqbal, Chupp, Simons, Farwell, Linder, Tsang, Grootes, Saunders, Lazzarini, Holmgren, Seymour, Cramer, Davis

2nd UP ROW: Weitkamp, Lamoreaux, Van Wechel, Geisse1, Leach, Grant, Fauska, Lesko, Bertram, Green, Wagner, Storm, Snover

Boпtom Row: Peterson, Swanson, Anderson, Hinn, Schmidt, Rohrbaugh, Wiest, Aniol, Gil, Von Lintig, Ikossi, Saling 


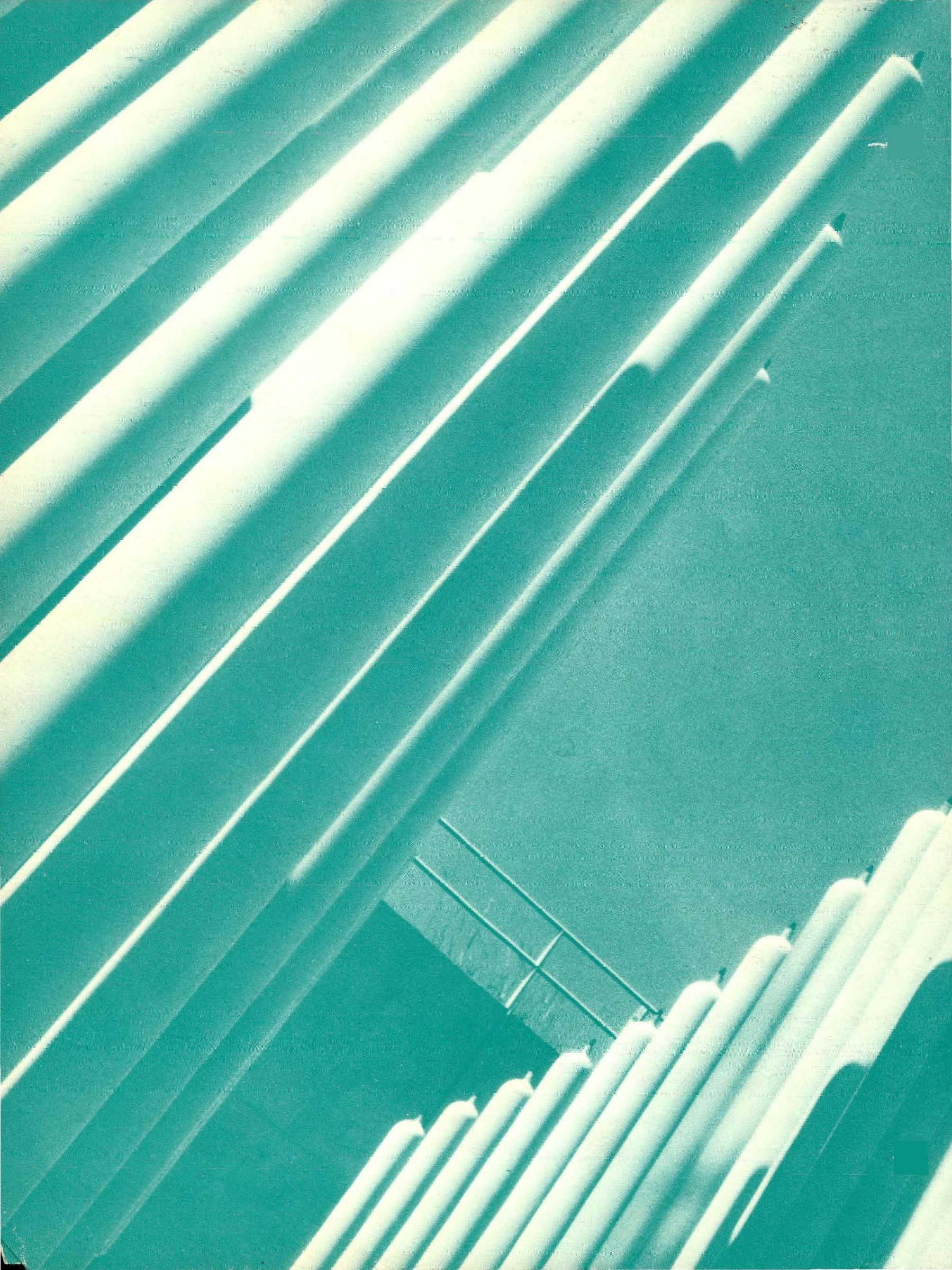

